\title{
Clustered Time Warp and Logic Simulation
}

\author{
Hervé Avril \\ School of Computer Science \\ McGill University, Montréal
}

A thesis submitted to the Faculty of Graduate Studies and Research in partial fulfillment of the requirements for the degree of Doctor of Philosophy in Computer Science.

Copyright (CDecember 1996 by Hervé Avril 
National Library

of Canada

Acquisitions and Bibliographic Services

395 Wellington Street Ottawa ON KIA ON4 Canada
Bibliothèque nationale du Canada

Acquisitions et services bibliographiques

395. rue Wellington

Ottawa ON K1A ON4

Canada
The author has granted a nonexclusive licence allowing the National Library of Canada to reproduce, loan, distribute or sell copies of this thesis in microform, paper or electronic formats.
L'auteur a accordé une licence non exclusive permettant à la Bibliothèque nationale du Canada de reproduire, prêter, distribuer ou vendre des copies de cette thèse sous la forme de microfiche/film, de reproduction sur papier ou sur format électronique.

The author retains ownership of the copyright in this thesis. Neither the thesis nor substantial extracts from it may be printed or otherwise reproduced without the author's permission.
L'auteur conserve la propriété du droit d'auteur qui protège cette thèse. $\mathrm{Ni}$ la thèse ni des extraits substantiels de celle-ci ne doivent être imprimés ou autrement reproduits sans son autorisation. 
A Jeannette et Cicide.

Mes deux grands amours. 


\section{Acknowledgments}

I would like to thank Prof. Carl Tropper, for his patience and his guidance during this research. Carl was more than an excellent research advisor, his encouragements, his care and his moral support were always very much appreciated. His financial assistance also made everything possible.

Thanks also to the CRIMI (Centre de Recherche Informatique de Montréal) for their financial support.

I am also very grateful to Darcy Brockbank for the fantastic impact he had on my programming skills. Without his teaching. the coding and the debugging phase of this research would have been very troublesome.

Thanks also go to the SOCS system wizards, and especially Luc Boulianne, Matther Sams, Ramesh Somalingan and hent Tse. They always rebooted biernat regularly and always very promptly.

Special thoughts go to my partners and friends at Hutchison: Darcy, Ivo. Martin. Ramesh. and Scott. It is an everyday wonderment to work with them.

I would like also to thank all the great people I have met at McGill. They have made these years very enjoyable and unforgettable. Some of them became very close friends. Special thoughts go to Alain (\#1), Andres (the Emperor), Azzedine (the Skydiver), Madhu (the Empress), Mohammed (Mr P.), Nasser (the baby-foot Tiger), and Rakesh (my snooker Master).

Many thanks also go to the DCS Villains: Husam, Pamir and Sarom. Despite the thousands of miles that keep us apart. we always managed to meet regularly and spend memorable moments together. 
Rien ne serait arrivé sans l'amitié que m'ont témoigné mes parisiens préférés. Carine, Isabelle. Jean-Jacques, Matthieu, et Norbert ont toujours su transformer des moments simples en de fantastiques souvenirs.

I would like also to thank Aaron for these long discussions we had. During troubled times, his presence and support helped me more than he could imagine.

Je voudrais aussi remercier Henri Lemmel. Son amour de la vie, son courage, et son intelligence ont fait de moi son plus fidèle disciple.

Des pensées toutes spéciales vont à Christianne, pour les doux et adorables moments que nous avons passé ensemble.

Mais avant tout, et par dessous tout, toute mon adoration et tous mes remerciements vont à mes parents. Leur amour, leurs encouragements, leur support, et la liberté qu'ils m ont donnés sont les plus beaux cadeaux que je n'ai jamais reçus. 


\section{Abstract}

In this research, the feasibility of using parallel discrete-event simulation techniques to run logic-level circuit simulations on general purpose distributed memory architectures is investigated. After studying the characteristics of VLSI models, we introduce Clustered Time Warp (CTW), a novel approach to parallel discrete event simulation. In CTW , the logical gates of a circuit are partitioned into clusters and the synchronization algorithm makes use of an optimistic approach between the clusters and a sequential approach within the clusters. We also present a new family of three space-based checkpointing algorithms for use with CTW. Results show that each checkpointing algorithm developed for CTW occupies a different point in the spectrum of possible trade-offs between memory usage and execution time.

We also present a dynamic load balancing algorithm developed for Clustered Time Warp which focuses on distributing the load of the simulation evenly among the processors and then tries to reduce inter-processor communications. We make use of a triggering technique based on the throughplit of the simulation system. Performance results show that by dynamically balancing the load, the throughput of the simulation system could be improved by more than a $100 \%$. No substantial improvement was observed on the overall simulation time when trying to minimize inter-processor communications. suggesting that load distribution is the most important factor to be taken into consideration in speeding up the simulation of digital circuits.

Furthermore. we examine the impact of partitioning and mapping on the performance and behavior of the Clustered Time Warp algorithm. We show that partitioning algorithms which try to minimize the number of cutsets between the partitions do not necessarily succeed in minimizing inter-processor communications. We also show that in our environment. load imbalance has a stronger effect than rollback overhead.

Finally, we study the problem of scalability encountered when using optimistic techniques. We show that the performance of Time Warp can greatly suffer from rollback explosions or when 
the dog chasing its tail phenomenon is observed. We also show that Clustered Time Warp is less sensitive to these phenomenons and as such, is more scalable than Time Warp. 


\section{Résumé}

Dans cette thèse. l'utilisation de techniques de simulations parallèles d'évènements discrets pour la simulation de circuits logiques sur des architectures à mémoire répartie est examinée. Après avoir étudié les caractéristiques des modèles de circuits intégrés. nous présentons Clustered Time Warp (CTW), une approche nouvelle à la simulation parallèle d'évenements discrets. Avec CTW, le partitionnement d'un circuit en plusieurs groupes est effectué et l'algorithme de synchronisation utilise une approche optimiste entre les groupes et une approche séquentielle à l'intérieur de ces groupes. Nous présentons aussi une nouvelle famille de trois algorithmes de sauvegarde d'états développés pour CTW. Les résultats obtenus montrent que chacun de ces algorithmes occupe un point différent dans le spectre des compromis entre l'usage de la mémoire et le temps d'exécution.

Nous présentons aussi un algorithme dynamique de balancement de charges développé pour Clustered Time Warp qui met d'abord l'accent sur la répartition égale de la charge de la simulation parmi les processeurs et essave par la suite de réduire la communication entre ces processeurs. Nous utilisons une technique de déclenchement basée sur le débit du système de simulation. Les résultats montrent qu'en balaçant les charges de façon dynamique. le débit du système de simulation a pu être amélioré de $100 \%$. Cependant. aucune amélioration substantielle du temps d'exécution n'a été observée lorsque la communication entre les processeurs à tenté d'être réduite. suggérant que le balancement de charges est le facteur le plus important à prendre en compte lors de l'accélération de la simulation de circuits logiques.

De plus. nous examinons limpact du partitionnement et de l'assignation des groupes aux processeurs sur la performance et le comportement de Clustered Time Warp. Nous montrons que les algorithmes de partitionnement qui essayent de minimiser le nombre de coupures entre 
les différentes partitions ne réussi nécessairement pas à minimiser la communication entre les processeurs. Nous montrons aussi que dans notre environnement, le manque de balance a un effet plus important que la surchage de travail occasionée par les retours en arrière.

Finallement, nous étudions le problème de dimensionnement rencontré lors de l'utilisation de techniques optimistes. Nous montrons que la performance de Time Warp peut souffrir grandement de l'explosion de retours en arrière ou lorsque le phénomène du chien courrant après sa queue est observé. Nous montrons aussi que Clustered Time Warp est moins sensible à de tels phénomènes et qu'ainsi, il répond mieux à des problèmes de plus grosse échelle. 


\section{Contents}

1 Introduction 1

1.1 Digital Simulators . . . . . . . . . . . . . . . . . . . 2

1.2 Asynchronous Algorithms $\ldots \ldots \ldots \ldots \ldots \ldots \ldots \ldots$

1.2.1 The Conservative Approach $\ldots \ldots \ldots \ldots \ldots \ldots$

1.2.2 The Optimistic Approach $\ldots \ldots \ldots \ldots . \ldots . \ldots . \ldots 8$

1.2.3 Extensions to the basic Time Warp algorithm . . . . . . . . . 14

1.2 .4 Hybrid approaches . . . . . . . . . . . . . . . 20

1.2.5 Comparing Time-Warp and the Chandy-Misra approach . . . . . 21

1.3 Logic Simulation and Parallel Discrete Event Simulation . . . . . . . . . . . . 22

1.4 Scope of our research . . . . . . . . . . . . . . . . . . . . . . . . . 24

2 On the Characteristics of Logic Circuit Simulation 26

2.1 The Simulation Model . . . . . . . . . . . . . . . . . . . . . 26

2.2 Discrete Event Simulation . . . . . . . . . . . . . . . . . 2 $2 \bar{\imath}$

2.3 Circuit Design . . . . . . . . . . . . . . . . . . . 30

2.4 Circuit Parallelism . . . . . . . . . . . . . . . . 31

2.4 .1 Related Results . . . . . . . . . . . . . . . . . . . 31

2.4 .2 Critical path analysis . . . . . . . . . . . . 33

2.4 .3 Experiments . . . . . . . . . . . . . . . . 35

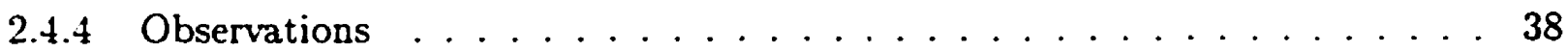

2.5 Workload Distribution . . . . . . . . . . . . . . 40

2.6 Workload Variability . . . . . . . . . . . . . . . . . 42 
2.7 Feedback Loops . . . . . . . . . . . . . . . . . . . . . . 44

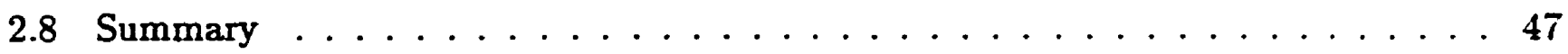

3 Clustered Time Warp $\quad 49$

3.1 Introduction . . . . . . . . . . . . . . . . . 49

3.2 The Clustered Time Warp Algorithm . . . . . . . . . . . . . 51

3.2 .1 Clusters . . . . . . . . . . . . . . . . . 51

3.2 .2 Events . . . . . . . . . . . . . . . . 51

3.2 .3 Scheduling . . . . . . . . . . . . . . . . . . 53

3.2 .4 Timezones . . . . . . . . . . . . . . . . 53

3.2.5 Logical Processes . . . . . . . . . . . . . . . . . . . 54

3.2 .6 Rolling back . . . . . . . . . . . . . . . . . 55

3.3 Pseudocode . . . . . . . . . . . . . . . . . . 56

3.3.1 Pseudocode for the Logical Process . . . . . . . . . . . . . . 56

3.3.2 Pseudocode for the Cluster Environment . . . . . . . . . . . . . 59

3.4 Example . . . . . . . . . . . . . . . . . . . . . . 60

3.4 .1 Receiving messages . . . . . . . . . . . . . 60

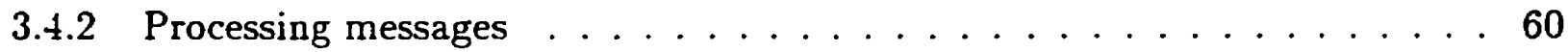

3.4 .3 Rolling back . . . . . . . . . . . . . . . . . . 6 61

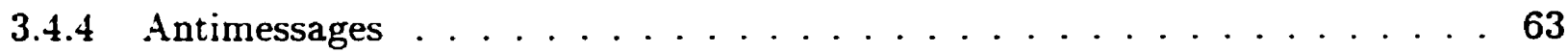

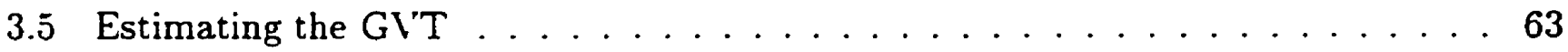

3.6 Space-based Checkpointing Techniques . . . . . . . . . . . . 65

3.6.1 Clustered Rollback. Clustered Checkpoint . . . . . . . . . . . . 65

3.6.2 Local Rollback, Clustered Checkpoint . . . . . . . . . . . . . . . . 66

3.6.3 Local Rollback. Local Checkpoint . . . . . . . . . . . . . . . . 66

3.7 Summary . . . . . . . . . . . . . . . . . . 67

4 Experiments and Results $\quad 70$

4.1 The Multiprocessor Environment $\ldots \ldots \ldots \ldots \ldots \ldots \ldots$

4.2 Simulation System . . . . . . . . . . . . . . . . . 72 
4.3 Experiments .............................. 74

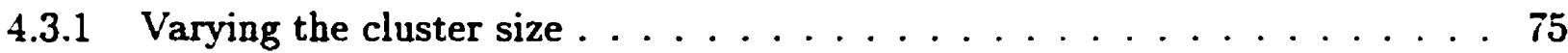

4.3.2 Varying the number of processors $\ldots \ldots \ldots \ldots$

4.3 .3 Speedup . . . . . . . . . . . . . . . . . . 84

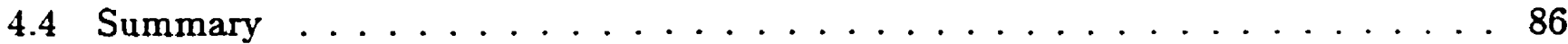

5 Dynamic Load Balancing $\quad 88$

5.1 Introduction . . . . . . . . . . . . . . . 88

5.2 Related results $\ldots \ldots \ldots \ldots \ldots \ldots \ldots \ldots$. . . . . . . . . . . 88

5.3 Discussion . . . . . . . . . . . . . . . . . 90

5.4 The Algorithm . . . . . . . . . . . . . . . . . . . 92

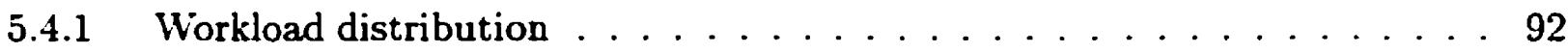

5.4 .2 Inter-Processor communication . . . . . . . . . . . . 93

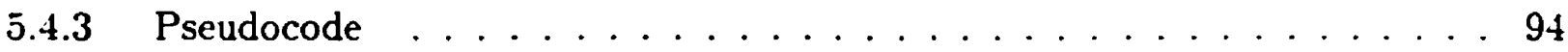

5.4 .4 Triggering the load-balancing algorithm $\ldots \ldots \ldots \ldots 6$

$5.5 \quad$ Metrics . . . . . . . . . . . . . . . . . . . 98

$5.5 .1 \quad 3:$ Workload distribution $\ldots \ldots \ldots \ldots$

$5.5 .2 \gamma$ : Inter-processor communication $\ldots \ldots \ldots \ldots$

$5.5 .3 \rho$ : Cancelled computation $\ldots \ldots \ldots \ldots \ldots$

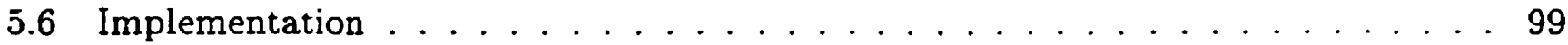

5.6 .1 Experiments . . . . . . . . . . . . . . . . 100

5.6 .2 Workload Distribution . . . . . . . . . . . . . . . . 100

5.6.3 Inter-processor Communications . . . . . . . . . . . . 106

5.6 .4 Memory requirements . . . . . . . . . . . . . . . 110

5.6.5 Throughput vs. Number of Processors . . . . . . . . . . . . 111

$5 . \bar{t}$ Summary . . . . . . . . . . . . . . . . . . . . . 112

6 Partitioning and Mapping 113

6.1 Related Results . . . . . . . . . . . . . . . . . . . 113

6.1.1 Classical partitioning methods for logic simulation . . . . . . . . 113 
6.1 .2 Adaptive Heuristic methods . . . . . . . . . . . . 115

6.1 .3 Other Approaches . . . . . . . . . . . . . . . . . 117

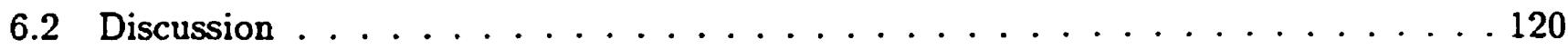

6.3 Sensitive cone partitioning . . . . . . . . . . . . . . 121

6.4 Experiments . . . . . . . . . . . . . . . . . . . 123

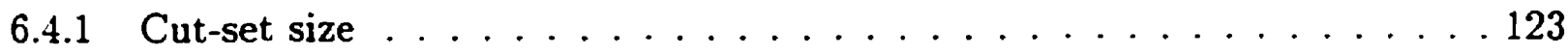

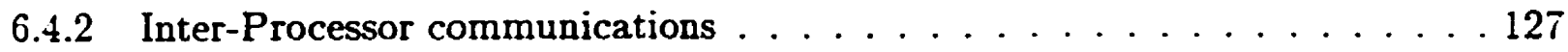

6.4 .3 Rollbacks . . . . . . . . . . . . . . . . . . . 128

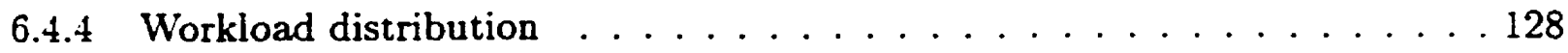

6.4 .5 Simulation time . . . . . . . . . . . . . . . . . . 129

6.4 .6 Mapping . . . . . . . . . . . . . . . . 130

6.5 Summary . . . . . . . . . . . . . . . . . . . 135

$\begin{array}{llr}7 & \text { Scalability and Stability } & 137\end{array}$

i.1 Related Resuits . . . . . . . . . . . . . . . . . . . . 137

7.2 Time Warp Hazards $\ldots \ldots \ldots \ldots$

i.2.1 Echoing . . . . . . . . . . . . . . . . . . . . . . . . . .

ז.2.2 Cascading Rollbacks . . . . . . . . . . . . . . . 140

7.2 .3 Dog chasing its tail . . . . . . . . . . . . . . . . . 142

7.2 .4 Discussion . . . . . . . . . . . . . . . . 143

7.3 Experiments . . . . . . . . . . . . . . . . . . 144

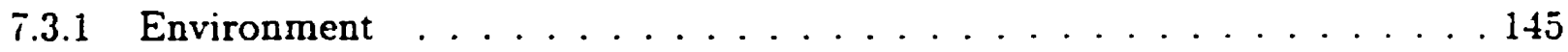

i.3.2 Results . . . . . . . . . . . . . . . . . . 150

7.4 Summary . . . . . . . . . . . . . . . . . . . 152

8 Conclusion $\quad 157$

8.1 Contributions . . . . . . . . . . . . . . . . . . . . .

8.2 Future Work . . . . . . . . . . . . . . . . . . 160

A Parallelism available in the ISCAS'89 Benchmark Circuits 162 
B Quantitative Results of Logic Simulations

C Quantitative Results of Partitioning Algorithms

D Quantitative Results of Queueing Network Simulations

E Relevant World Wide Web sites 


\section{List of Figures}

1.1 Time Warp Environment of a Logical Process . . . . . . . . . . . . . 10

1.2 Time Warp Environment of the LP after message with timestamp 39 was processed 10

1.3 Time Warp Environment of the LP after message with timestamp 34 was cancelled 10

1.4 GVT Computation with 5 processors $\ldots \ldots \ldots \ldots \ldots \ldots$

1.5 Time Warp Environment of the LP after message at 34 was cancelled and if lazy

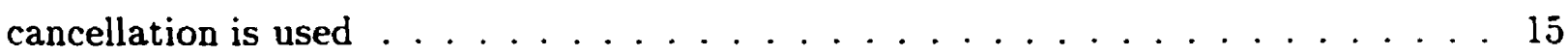

2.1 Example of a small circuit $\ldots \ldots \ldots \ldots \ldots \ldots$

2.2 Initial state of the discrete event simulation $\ldots \ldots \ldots \ldots \ldots$

2.3 The simulation system at $t_{s}=12$ and $t_{s}=14$ respectively $\ldots \ldots \ldots \ldots 29$

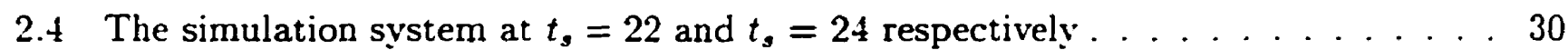

2.5 Building blocks of the Intel $\mathrm{P} 6 \ldots \ldots \ldots \ldots \ldots \ldots \ldots$

2.6 Space-time diagram of the event dependence graph . . . . . . . . . . . . 34

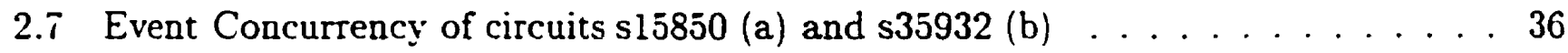

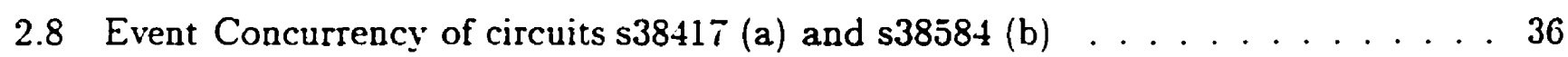

2.9 Synchronous and Asynchronous Average Parallelism . . . . . . . . . . . 39

2.10 Synchronous and Asynchronous Relative Parallelism . . . . . . . . . . . . . 39

2.11 Workload distribution for circuits $s 15850$ (a) and $s 35932$ (b) . . . . . . . . 40

2.12 Workload distribution for circuits $\mathrm{s} 3841 \mathrm{i}$ (a) and $\mathrm{s} 38584$ (b) . . . . . . . 40

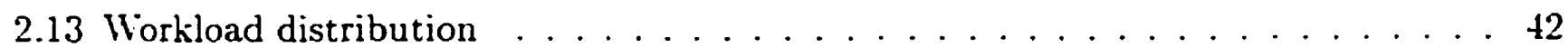

2.14 Workload Variability . . . . . . . . . . . . . . . . . . . . 43

2.15 Pseudocode of the main procedure of the cycle inventory algorithm . . . . . . 44

2.16 Pseudocode of the ModifiedBFS procedure of the cycle inventory algorithm . . . . 45 


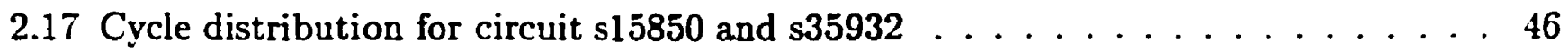

2.18 Cycle distribution for circuits $s 38417$ and $s 38584 \ldots \ldots \ldots \ldots \ldots \ldots$

3.1 General Circuit Structure $\ldots \ldots \ldots \ldots \ldots \ldots$

3.2 Cluster Structure $\ldots \ldots \ldots \ldots \ldots \ldots \ldots \ldots \ldots$

3.3 The Logical Process is about to process event $e \ldots \ldots \ldots \ldots \ldots$

3.4 The LP is told by the CE to rollback to time $t_{\text {rbk }} \ldots \ldots \ldots \ldots \ldots$

3.5 The cluster has received event $e \ldots \ldots \ldots \ldots \ldots \ldots \ldots$

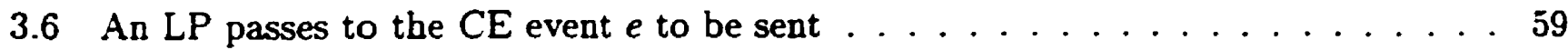

3.7 (a) The system starts and (b) message $m_{1}$ is received for $L P_{1} \ldots \ldots \ldots \ldots$

3.8 (a) $L P_{1}$ processes $m_{1}$ and (b) $L P_{2}$ and $L P_{3}$ process $m 2 . m 3$ and $m 4 \ldots \ldots \ldots 6$

3.9 (a) $L P_{1}$ receives straggler $m_{5}$ and (b) $L P_{2}$ and $L P_{3}$ are rolled back and $m_{4}$ is discarded ......................... 61

3.10 (a) $L P_{2}$ coasts forward and (b) the cluster resumes and proceeds normally . . . 62

3.11 (a) $m_{5}$ is annihilated by its antimessage, the cluster rolls back, and (b) $m_{3}$ is

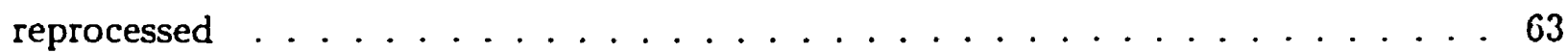

3.12 Operations performed by the LP once a new GVT estimate is calculated . . . . 64

3.13 Checkpointing techniques . . . . . . . . . . . . . . . . 68

4.1 The GP1000 shared-memory multiprocessor . . . . . . . . . . . i1

4.2 Example of a string partitioning for circuit s27 (cluster size $=4) \ldots \ldots \ldots$ it

4.3 Memory vs. Number of clusters per processor (circuit s35932) $\ldots \ldots \ldots \ldots$. . . . 6

4.4 Memory vs. Number of clusters per processor (circuit s38584) $\ldots \ldots \ldots$ it

4.5 Simulation time vs. Number of clusters per processor (circuit s35932) $\ldots \ldots$. . 78

4.6 Simulation time vs. Number of clusters per processor (circuit s38584) . . . . . . 79

4.7 Larger checkpoint interval does not always imply smaller memory usage . . . . . 80

4.8 Number of states vs. Number of processors (circuit s35932) . . . . . . . . 81

4.9 Number of states vs. Number of processors (circuit s38584) . . . . . . . . . 81

4.10 Memory usage vs. Number of processors (circuit s35932) . . . . . . . . . . 82

4.11 Memory usage vs. Number of processors (circuit s38584) $\ldots \ldots \ldots \ldots$. . . . 83 
4.12 Simulation time vs. Number of processors (circuit s35932) $\ldots \ldots \ldots \ldots$

4.13 Simulation time vs. Number of processors (circuit s38584) $\ldots \ldots \ldots \ldots$. . . . 84

4.14 Speedup observed for circuit $s 35932 \ldots \ldots \ldots \ldots \ldots \ldots \ldots$

4.15 Speedup observed for circuit $\mathbf{s} 38584 \ldots \ldots \ldots \ldots \ldots \ldots$

4.16 Space-Time performance results for circuit $s 35932 \ldots \ldots \ldots \ldots$. . . . . . 87

4.17 Space-Time performance results for circuit s38584 $\ldots \ldots \ldots \ldots$. . . . . . 87

5.1 Same number of events, different virtual time progress . . . . . . . . . . . . . 92

5.2 Pseudocode of the load balancing algorithm . . . . . . . . . . . 95

5.3 Throughput during a transfer $\ldots \ldots \ldots \ldots \ldots \ldots \ldots \ldots$

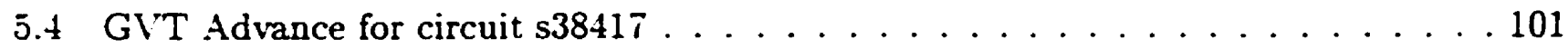

5.5 GVT Advance for circuit s38584 . . . . . . . . . . . . . . . . . 102

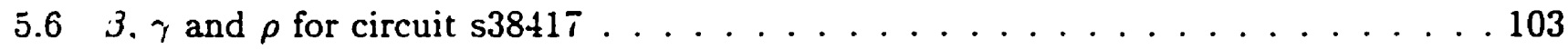

$5.7 \quad 3 . ;$ and $\rho$ for circuit $\mathrm{s} 38584 \ldots \ldots \ldots \ldots \ldots$

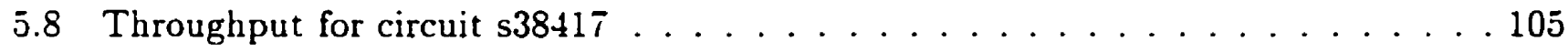

5.9 Throughput for circuit $\mathbf{s} 38584 \ldots \ldots \ldots \ldots \ldots$

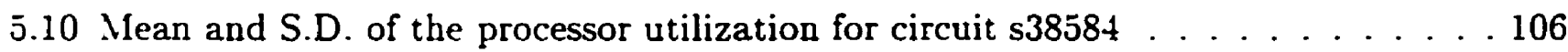

5.11 Effect on $\gamma$ for circuit $\mathrm{s} 38+1 \mathrm{\tau}$ if IPC is minimized $\ldots \ldots \ldots 10 \ldots \ldots$

5.12 Effect on, for circuit $\mathbf{s} 38584$ if IPC is minimized $\ldots \ldots \ldots \ldots$. . . . . . . . .

5.13 Effect on $\rho$ for circuit $\mathrm{s} 3841 \mathrm{t}$ if IPC is minimized $\ldots \ldots \ldots 108 \ldots \ldots$

5.14 Effect on $\rho$ for circuit $\mathrm{s} 38584$ if IPC is minimized $\ldots \ldots \ldots . \ldots 108$

5.15 Effect on the GVT Advance for circuit s38417 if IPC is minimized . . . . . . . . 109

5.16 Effect on the GVT Advance for $\mathrm{s} 38584$ if IPC is minimized . . . . . . . . . . . . 109

5.17 Peak Memory usage for circuit s38417 . . . . . . . . . . . . . . . 110

5.18 Peak Memory usage for circuit s38584 . . . . . . . . . . . . . . . 110

5.19 Improvement of the Throughput . . . . . . . . . . . . . . 111

6.1 Effect of introducing sensitivity to an output cone partitioning algorithm . . . . 121

6.2 Pseudocode of the sensitive output cone partitioning algorithm . . . . . . . . . 122

6.3 Relative size of the cut-set for circuit s38584 . . . . . . . . . . . . . . 125 


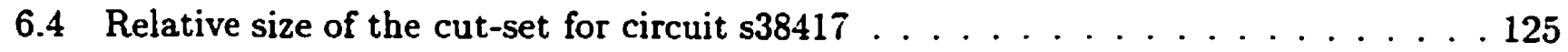

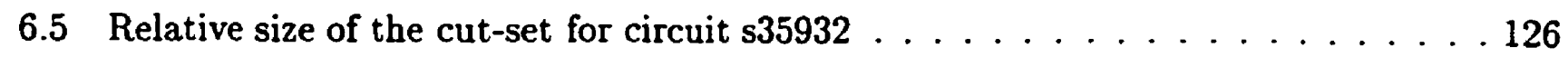

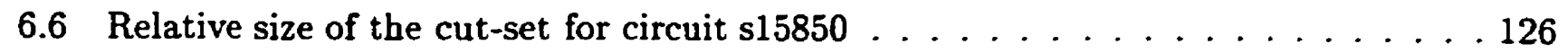

6.7 Effect of partitioning on inter-processor communications for circuit s38584 $\ldots 127$

6.8 Effect of partitioning on the number of rollbacks for circuit s38584 $\ldots \ldots \ldots \ldots$

6.9 Effect of partitioning on the load balance for circuit s38584 . . . . . . . . . 129

6.10 Effect of partitioning on the simulation time for circuit $s 38584 \ldots \ldots \ldots \ldots$

6.11 Example of the Natural and the Random mapping strategies . . . . . . . . . 131

6.12 Effect of mapping on the interprocessor communications (circuit s38584) $\ldots 132$

6.13 Effect of mapping on the workload distribution (circuit s38584) $\ldots \ldots \ldots$

6.14 Effect of mapping on the rollbacks (circuit s38584) . . . . . . . . . . . 134

6.15 Effect of mapping on the simulation time (circuit $\mathrm{s} 38584$ ) . . . . . . . . . 135

7.1 Connection graph of the nodes involved in the echoing phenomenon . . . . . 139

7.2 Connection graph of the nodes involved in the cascading phenomenon . . . . . 141

7.3 Connection graph of the nodes involved in the 'dog chasing its tail' phenomenon .142

i.4 Journey of a computation wave (a) and a cancellation wave (b) . . . . . . . 144

i.5 The pipeline model . . . . . . . . . . . . . . . . . . . . . . . 145

T.6 The hierarchical network model . . . . . . . . . . . . . . . . . . 146

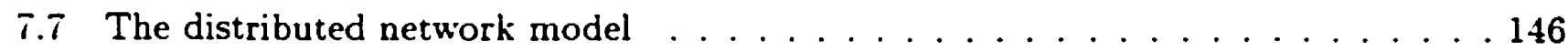

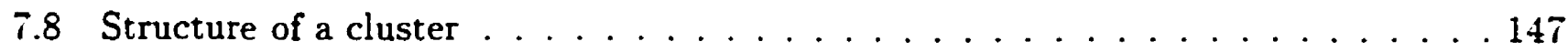

7.9 Stimulation of the pipeline model . . . . . . . . . . . . . . . . . . . 149

7.10 Throughput for the pipeline model $\ldots \ldots \ldots \ldots \ldots \ldots$

T.11 Rollbacks for the pipeline model . . . . . . . . . . . . . . . . . . . . . . . 154

7.12 Memory usage for the pipeline model . . . . . . . . . . . . . . 154

7.13 Throughput for the hierarchical model . . . . . . . . . . . . . . 155

7.14 Rollbacks for the hierarchical model . . . . . . . . . . . . . . . . 155

7.15 Memory usage for the hierarchical model . . . . . . . . . . . . . . 155

7.16 Throughput for the distributed model . . . . . . . . . . . . . . 156

7.17 Rollbacks for the distributed model $\ldots \ldots \ldots \ldots \ldots \ldots \ldots$ 
7.18 Memory usage for the distributed model $\ldots \ldots \ldots \ldots \ldots$

A.1 Average parallelism measured in the ISCAS'89 Benchmark circuits . . . . . . . 162 


\section{List of Tables}

2.1 IEEE Standard Logic System . . . . . . . . . . . . . . 27

2.2 ISCAS'89 Benchmark circuits characteristics . . . . . . . . . 35

2.3 Synchronous Parallelism . . . . . . . . . . . . . . 37

2.4 Asynchronous Parallelism $\ldots \ldots \ldots \ldots \ldots \ldots$

4.1 Arithmetic Operation Times $(\mu \mathrm{s}) \ldots \ldots \ldots \ldots \ldots \ldots \ldots$

4.2 Message Transmission time between nodes . . . . . . . . . . . 72

4.3 Circuit $\mathrm{s} 35932$ and $\mathrm{s} 38584 \ldots \ldots \ldots \ldots \ldots \ldots \ldots$

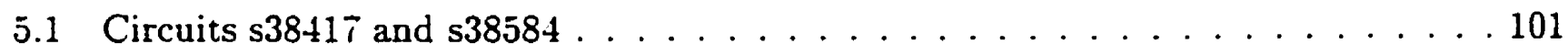

6.1 Description of the circuits studied in this chapter $\ldots \ldots \ldots \ldots \ldots \ldots$

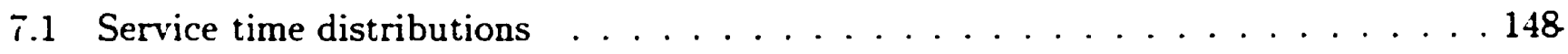

A.1 Parallelism measured in the ISCAS'89 Benchmark circuits . . . . . . . . 163

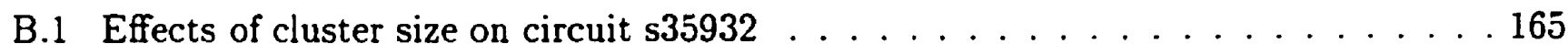

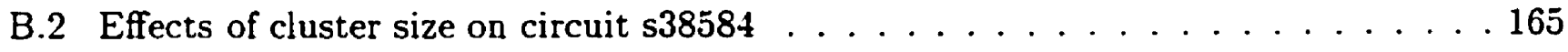

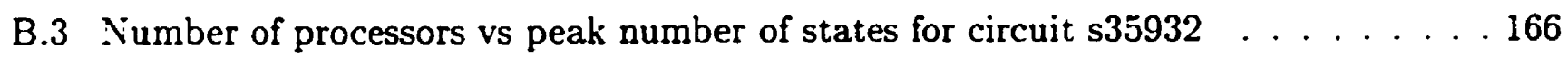

B.4 Number of processors vs memory for circuit $\mathbf{s 3 5 9 3 2 \ldots \ldots \ldots \ldots} \ldots \ldots$

B.5 Number of processors vs simulation time for circuit $s 35932 \ldots \ldots \ldots 6$

B.6 Number of processors vs peak number of states for circuit s38584 . . . . . . 167

B.7 Number of processors vs memory for circuit s38584 . . . . . . . . . 167

B.8 Number of processors vs simulation time for circuit s38584 $\ldots \ldots \ldots \ldots$

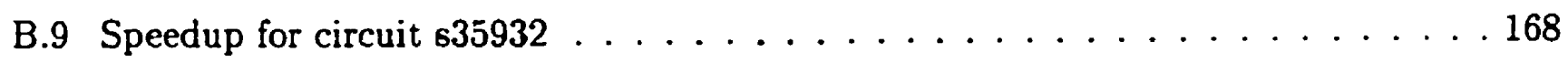




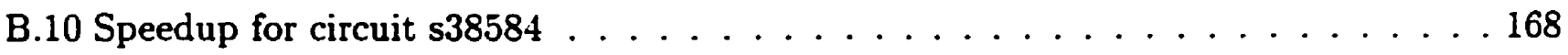

C.1 Effect of the partitioning on size of the cutset $\ldots \ldots \ldots \ldots \ldots \ldots$

C.2 Effect of partitioning on the simulation of circuit $s 15850 \ldots \ldots \ldots \ldots$

C.3 Effect of partitioning on the simulation of circuit $s 35932 \ldots \ldots \ldots \ldots \ldots$

C.4 Effect of partitioning on the simulation of circuit $s 38417 \ldots \ldots \ldots \ldots$

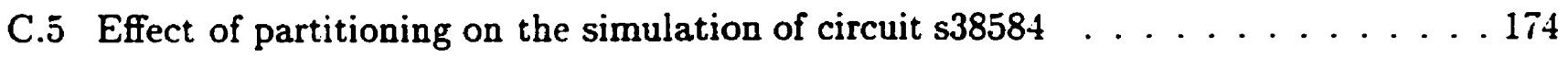

C.6 Effect of Random Mapping on the simulation of circuit s38584 . . . . . . . 175

C.7 Effect of Vertical Natural Mapping on the simulation of circuit s38584 . . . . 175

C.8 Effect of Horizontal Natural Mapping on the simulation of circuit s38584 . . . 175

D.1 Pipeline model - Time $\operatorname{Warp} \ldots \ldots \ldots \ldots \ldots \ldots \ldots$. . . . . . . . . . . . . . .

D.2 Pipeline model - Clustered Time Warp . . . . . . . . . . . . . 17i

D.3 Hierarchical network model - Time Warp . . . . . . . . . . . . . . 178

D.t Hierarchical network model - Clustered Time Warp . . . . . . . . . . . 178

D.5 Distributed network model - Time Warp . . . . . . . . . . . . . . 179

D.6 Distributed network model - Clustered Time Warp . . . . . . . . . . . 179 


\section{Chapter 1}

\section{Introduction}

In the late 70 s. Intel was working on the 8086. a new microprocessor which led the way in the computer revolution in the $80 \mathrm{~s}$. Back then. packing 29,000 transistors on the same silicon chip and producing it in large numbers was a great achievement.

Then came the Motorola 68000 containing about 70.000 transistors. and the Intel 80286 with 130.000 transistors. The 90 s gave birth to the Motorola 68040 and the Intel 80486 with about 1.2 million transistors each. Today, the PowerPC 603E has about 2.6 million transistors, the Intel Pentium P6 packs 5.5 million transistors in its CPL core. and the R10000 integrates 6.8 million transistors.

In the last 20 years. VLSI circuits had their size increased by at least 2 orders of magnitude. Their design is a real challenge and their testing a major issue. Simulation execution time of VLSI circuits is proportional to both the size of the circuit and the number of test patterns. Since test patterns are themselves proportional to the size of the circuit. the total simulation time is then proportional to the square of the circuit size. A circuit simulation can therefore easily take hours. days. or even weeks. Infortunately. hardware engines for logic simulation. such as the Yorktown Simulation Engine from IBM or the Logic Evaluator from ZYCAD. have not really followed the trend, and they are extremely expensive. Hence there exists a need for faster. more flexible and scalable distributed logic software simulators which can run on multi-purpose architectures. 


\section{$1.1 \quad$ Digital Simulators}

When simulating digital circuits. designers first create a model of the system under both faulty and fault free conditions. Input test vectors are then generated in order to exercise the model. The output response is analyzed to evaluate the performance and the functionality of the circuit.

There are different levels of abstraction under which the simulation process is carried out. Each of these levels emphasizes an aspect of the circuit. Higher levels of simulation provide information regarding the functional aspects of the system, whereas lower levels of simulation concentrate more on the performance of the circuit.

There are 8 major levels of simulations used in the design process of digital systems [Russ85]:

1. At the behavioral level, a model of the circuit is created to characterize the behavior of a circuit regardless of its internal structure.

2. A register transfer level simulator deal with registers. I/O, ALL, buses, etc.

3. Functional level simulators deal with systems specified in terms of major building blocks and their interconnection.

4. Only discrete logic levels are used in a gate level simulator whose purpose is to validate the logical behavior of the circuit. Basic logic gates are provided by the simulator (eg: NAND. OR. NOR, etc.). In some cases, flip-flops and shift-registers are also available.

5. In a switch level simulator, MOS transistors are modelled as a voltage controlled switch. The advantage of modelling a group of MOS transistors rather than a simple logic gate is that MOS devices have a bilateral switching characteristic which allow them to perform complex logic functions when grouped together. Developed by Bryant, MOSSIM was one of the first switch-level simulators developed [Brya84].

6. Timing level simulators (such as RSIM [Term83] and CRYSTAL [Oust83]) are relatively new. These are similar to circuit level simulators except for the fact that they use simpler models and relaxed simulation methods. Therefore, the designer can simulate circuits faster with an accuracy comparable to circuit level simulators. 
7. Circuit level is usually the lowest level of abstraction in which the circuit designer checks the different electrical characteristics of a group of transistors which are generally a small subset of the entire circuit. SPICE [Mukh86] is one example of a simulator performing circuit analysis.

8. At the device level, various aspects of the fabrication process on device parameters are examined. The designer is usually not involved at this stage.

Simulation can be used to check the functionality of a circuit (function verification), to detect faults (fault simulation), to monitor switching anomalies (spike and hazard analysis), to determine the delays (timing analysis), to find out about the effects of external factors. such as temperature on the circuit (sensitivity analysis), and to also evaluate different alternative designs.

Briner [Brin90] enumerates seven different paradigms used by parallel discrete-event simulators:

- The support paradigm decomposes the simulator into a series of functional units which are then mapped onto the parallel machine (just like a pipeline).

- The replicated paradigm. in which a stochastic simulation is replicated.

- The data parallel paradigm in which each processor performs the same computation but with different data.

- The data-flow paradigm.

- The central dispatcher paradigm. in which there exists a central single event queue shared by all the processors working on the model evaluation.

- The case of the Massively Parallel Computer which has only one instruction stream for all the processors.

- And finally, the asynchronous algorithms, in which synchronization techniques must be used in order to ensure the correctness of the simulation which either runs on a shared-memory or a distributed-memory multi-processor architecture. 
Only the asynchronous algorithms stand out as a good candidate for digital simulation on general purpose parallel machines. In the case of the support paradigm, pipelining is not appropriate since a simulator can only be decomposed into a very limited number of functional units, therefore limiting the potential speed-up on massive parallel machines. In the case of logic simulation, the time needed for the model evaluation is actually very small, therefore the central dispatcher paradigm offers very little use, simply because of all the congestion the central queue would create. The replicated paradigm can only be effective for fault simulation where a large number of independent vectors need to be simulated. In the case of design verification where minimizing the simulation time of a single input vector is a priority, the replicated paradigm becomes much less effective. However. the replicated paradigm does not solve the problem of large simulation models that cannot fit on a single processor unit due to the limited amount of memory resources. Other paradigms require parallel machines with a specific architecture or put too many restrictions on the simulation model.

\subsection{Asynchronous Algorithms}

One way to perform a simulation on a parallel environment is by using a time step technique in which all the processes are strictly synchronized with respect to physical time. This technique is easy to implement but it does not exploit all the parallelism inherent in a VLSI model. Since only a very small number of gates in a circuit are active at the same time, a lot of processing power is lost.

Lamport [Lampi8] suggested the notion of logical clock which is a monotonically increasing counter. Each process maintains its own logical clock whose value is used to place a timestamp in the messages sent by the process. He also introduced the happened-before relation. known as causal ordering, which is based on two simple intuitive points [Coul94]:

1. If two events occur at the same process, then they occur in the order in which the process observes them.

2. Whenever a message is sent between processes, the event of sending the message occurred before the event of receiving the message. 
The notion of logical time introduced by Lamport constitutes the major milestone upon which rests the whole family of asynchronous algorithms. With this paradigm, physical time can now be completely abstracted since the only thing that matters is whether causally related messages are processed in the correct order. This is where asynchronous algorithms come into the picture. Their purpose is to preserve the correctness of the system with respect to causality.

There exist two major classes of asynchronous algorithms. The conservative approach pioneered by Chandy, Misra [Chan79] and Bryant [Brya77] and the optimistic approach developed by Jefferson [Jeff85]. Other algorithms such as Space-Time and Local Time Warp have also emerged.

\subsubsection{The Conservative Approach}

In the Chandy-Misra-Bryant approach, a physical system, composed of independent and interacting entities (Physical Processes or PPs), is modelled as a logical system composed of Logical Processes (LPs). The topology of the physical system is represented in the logical system and interactions between the physical processes are modelled by timestamped messages exchanged by the logical processes. The timestamp of each message corresponds to the arrival time of the event at the receiving LP. Each logical process has a local clock (Local Simulation Time or LST) which is the timestamp of the last message sent by the LP. Simulation correctness is ensured by the fact that events are processed by each LP in strictly increasing order of their timestamps. therefore preserving the causality principle, as defined in [Reed88]:

The behavior of a node $P$ at simulated time $T$ cannot be influenced by any information transmitted to it after time $T$.

Avoiding or detecting/breaking deadlocks is the main problem conservative algorithms have to solve. The necessary and sufficient condition for the existence of a deadlock is given by Peacock et al. [Peaci9]:

A deadlock exists if and only if there is a cycle consisting of empty links which all have the same link time ${ }^{1}$, and nodes which are all blocked because of these links.

\footnotetext{
${ }^{1}$ The link time is the lower bound on the arrival time of the next message on a link
} 
Several solutions to the deadlock problem have been proposed in the literature. Chandy and Misra [Chan79] suggested a null message technique in order to avoid deadlocks. In their approach, null messages are sent on all of the output links of an LP each time the LP sends an event message. Null messages are timestamped with the same time value as the event message but do not contain the message fields. This way the receiving LPs know the lower bound of the timestamp of the next messages that will be put in their input queues. This method, as well as most of the other asynchronous algorithms, needs a non-zero service time in order to succeed. The disadvantage of this technique is that a large number of messages are generated, which can easily cripple the simulation system due to the communication overhead. Misra [Misr86] proposes several alternatives to this problem. For example, he suggests that LPs wait for a while before sending out null messages (using a time out). He also presents a demand driven technique in which LPs send query messages to their predecessors in order to get a new lower bound on the time of the next event to come.

Another variant of the null message technique has been proposed by Su and Seitz [Su89] in their lazy message technique. They suggest postponing the sending of null messages in the hope of decreasing the volume of messages. Su and Seitz performed experiments on a variety of logic networks. Their performance results show that their algorithm performs better than the basic form of the CMB algorithm in the case of small digital circuits (a 116-gate multiplier). However. if the circuit becomes larger, the difference becomes less significant. The reason behind this is that smaller circuits contain fewer elements per processor, the processors have less work to perform. and more null messages need to be sent in order to avoid deadlocks.

Nicol [Nico88] proposed a slightly different technique to avoid deadlock in which he makes use of a future list. In his approach, each time a group of jobs arrive. an LP. based on its service time. computes the lower bound of the receiving time of the next job to be sent to other LPs. Experiments were performed on a shared-memory machine and results show that performance reaches its optimal level when the simulation load is heavy or moderate. Furthermore, he points out that service time has a strong effect on speedup (the higher the lookahead ${ }^{2}$, the better the

\footnotetext{
${ }^{2}$ The lookahead for a process $p$, in a given state, is equal to the minimum timestamp increment (difference between the output and the input timestamps) for the earliest future output sent by $p$ [Jha94]. Lookahead is also sometimes characterized by an average time value [Lin90c]
} 
speedup).

Many different algorithms have also been proposed in the literature for the detection and the breaking of deadlocks [Elma86. Chan83, Nata86, Liu90].

Reed and Malony [Reed88, Reed88b] report poor performance results regarding the conservative simulation of queueing network models and conclude:

... the Chandy-Misra technique is not a viable approach to parallel simulation of queueing network models.

They base their claims on the observation that the overhead for deadlock detection and recovery is too large. especially when the nodes do little computation.

Groselj and Tropper [Gros88. Gros91] proposed a local deadlock resolution algorithm. called the Time of Next Event algorithm (TNE). Based on the shortest path algorithm, their technique computes the greatest lower bound on the input links of processes located on the same processor. It therefore becomes possible to unblock LPs having input messages bearing a smaller timestamp than the smallest time-of-next-event estimate of their empty input links. To break inter-processor deadlocks. Groselj and Tropper [Gros91] extended their original algorithm and proposed a distributed deadlock breaking algorithm called the Distributed Limited version of the TNE algorithm (or DLT.NE). Boukerche and Tropper [Bouk91] assessed the speedup obtained by $T N E$ relative to a sequential simulator. In their experiments, they selected a queueing network model with an FCFS service discipline as a benchmark. With a model of about 400 logical processes. they observe a significant speedup up to 10 processors. The improvements quickly reach a ceiling as the number of processors increases since TNE can unblock fewer LPs as their population per processor decreases. Boukerche [Bouk95] compares the TNE approach to the null message algorithm developed by Chandy and Misra [Chan79]. TNE appeared to be 2 to 5 times faster than the CMI approach. The best results were obtained when large network models were used and the simulation was ran on an average number of processors.

Lubachevsky [Luba88] proposed the bounded lag algorithm. In his approach, two events are allowed to be processed concurrently if their timestamp difference is smaller than a given constant. Formally, if events $e_{1}$ and $e_{2}$ are processed concurrently, then $\left|\tau\left(e_{1}\right)-\tau\left(e_{2}\right)\right| \leq B$ where $\tau(e)$ is 
the timestamp value of event $e$ and $B$ is a known positive constant. The algorithm iteratively calculates the current smallest timestamp and broadcasts its value to all of the processors. Then on each processor, events which satisfy the bounded lag restriction are processed. The efficiency of this algorithm depends upon the value of $B$. If the constant is too small, very little parallelism will be achieved. Nevertheless. Lubachevsky reports that he was able to write an efficient parallel Monte-Carlo simulation.

Wagner, Lazowska and Bershad [Wagn89] suggested a new technique for shared-memory parallel simulation called lazy blocking avoidance. In their approach, the responsibility for breaking a deadlock is delegated to the kernel which uses an idle processor without performing a context switch. Their technique is similar to the null-message based deadlock avoidance algorithm [Misr86], the equivalent of an eager blocking avoidance mechanism. The difference lies in the fact that the runtime system utilizes idle processors to propagate lookahead values on a demanddriven basis. This technique has the advantage of using only idle physical resources. The authors have implemented their technique into the Synapse [Bers88] system. and observed that a conservative approach. when tailored on a shared-memory machine. can vield a better speedup than either a deadlock detection algorithm or an eager blocking avoidance algorithm.

\subsubsection{The Optimistic Approach}

Optimistic techniques were first suggested by Jefferson in [Jeff85] in which he proposed Virtual Time, a new paradigm for distributed computation. and introduced the Time Warp mechanism. In Time Warp. each logical process maintains its own local virtual clock. The local virtual time (or LVT) at an LP is the value of the virtual clock, equal to the value of the timestamp of the next message in the input queue awaiting to be processed. Each LP has a single input queue and a single output queue in which messages are sorted in order of increasing timestamp. An LP assumes that it will not receive any message whose timestamp (receive time) is smaller than the lowest timestamp of all of the messages already received by the LP and waiting to be serviced. Nevertheless. it is possible for an LP to receive a message with a timestamp whose value is lower than that of its virtual clock. Such messages are referred to as stragglers. To preserve the causality principle, a rollback mechanism is needed in order to restore the LP to a state prior 
to the timestamp of the straggler. Therefore, the LP must keep a copy of its state each time it is about to change it (ie: each time the LP is about to consume a message). The LP must also keep track of all the messages it sends in order to be able to cancel wrong computations when a straggler is received. Suppose the LP, just before receiving the straggler, sent messages to its successors. In this case, all the computation triggered by these wrong messages must also be cancelled. Jefferson proposes to use antimessages which are copies of the wrong messages sent but bearing a negative sign (normal messages all have a positive sign). The purpose of an antimessage is either to annihilate its peer message, in the case it has not been processed yet, or to force the LP which has consumed the message to rollback to a state prior to the timestamp of that antimessage. To this end, LPs will therefore keep in their output queue a copy of each message they send and change their sign into a negative sign. In the Time Warp mechanism. an LP is then composed of a process name which is unique in the entire simulation system, a local virtual clock. a state. a state queue. an input queue, and an output queue. Messages have 6 fields: the send time. the receive time, the name of the sending LP, the name of the receiving LP. the sign (+ for messages or - for antimessages), and data.

Figure 1.1 contains an example of the structure of a logical process in a Time Warp environment. In this example, the LP is about to process the message whose receive time is 39 . Figure 1.2 shows the state of the system once the LP has processed the message. Notice that the state of the LP has been saved and an antimessage has been added to the output queue. Figure 1.3 shows the environment of the LP after the message with timestamp 34 has been cancelled by an antimessage. The LP rolled back to the state saved at time 34 and antimessages whose sent time were higher than 34 were sent. 


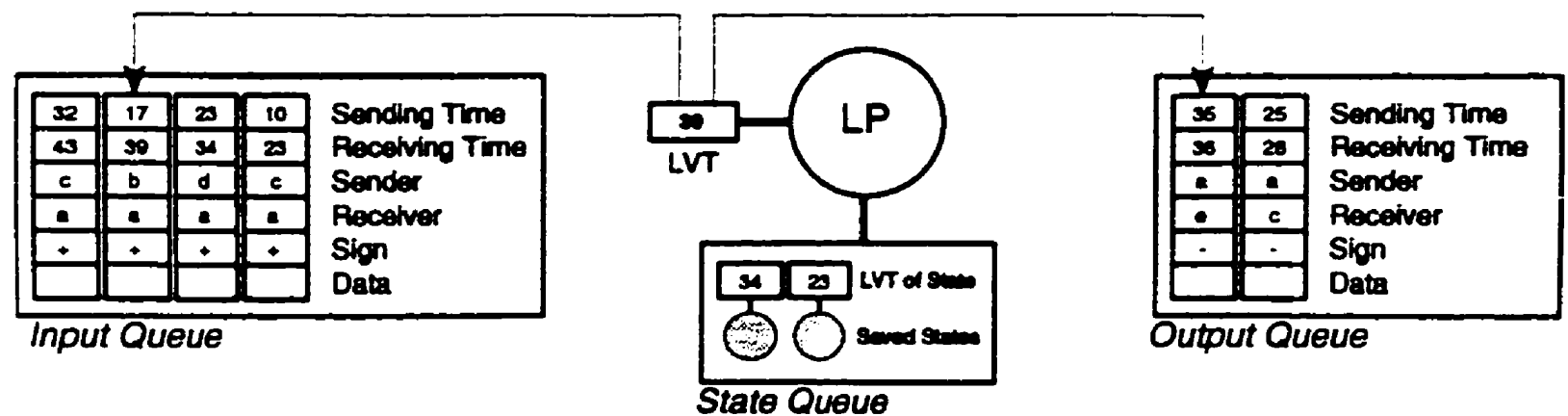

Figure 1.1: Time Warp Environment of a Logical Process

\begin{tabular}{|c|c|c|c|c|}
\hline 32 & 17 & 23 & 10 & \multirow{6}{*}{$\begin{array}{l}\text { Sending Time } \\
\text { Receiving Time } \\
\text { Sender } \\
\text { Pleceiver } \\
\text { Sign } \\
\text { Data }\end{array}$} \\
\hline 4 & 30 & 34 & 20 & \\
\hline c & 0 & d & c & \\
\hline 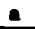 & $a$ & - & 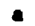 & \\
\hline - & $\bullet$ & 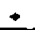 & + & \\
\hline & & & & \\
\hline
\end{tabular}

Input Queve

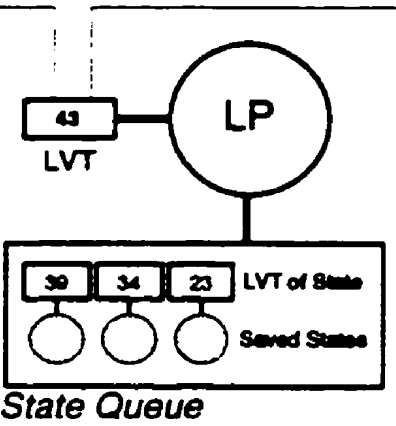

Figure 1.2: Time Warp Environment of the LP after message with timestamp 39 was processed

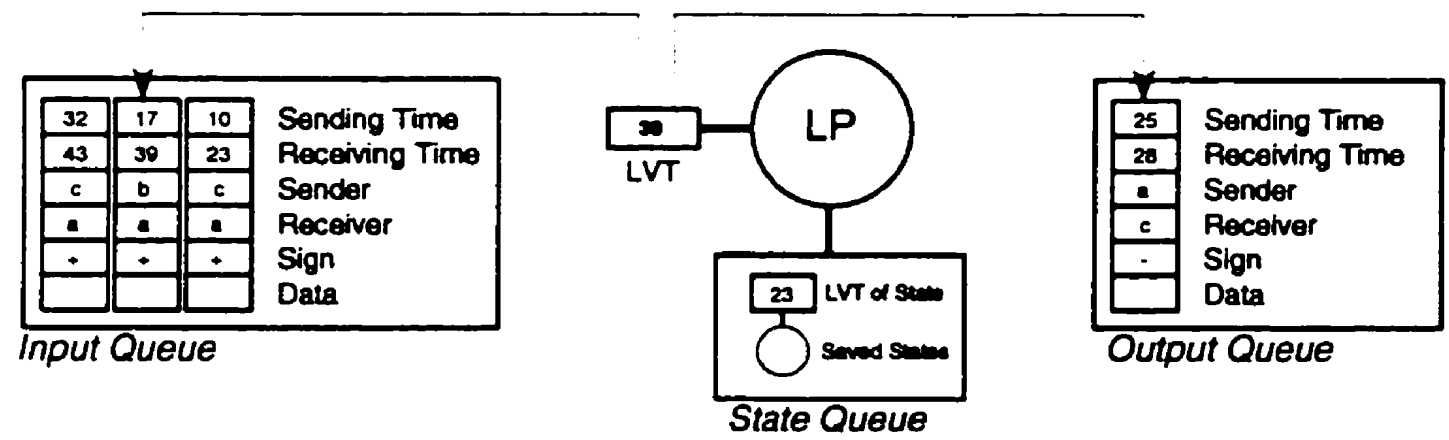

Figure 1.3: Time Warp Environment of the LP after message with timestamp 34 was cancelled 


\section{Fossil collection and GVT}

One of the most delicate issues in Time Warp is the fossil collection mechanism. The system must be able to discard, for memory reuse, all of the messages and states that are too old. Jefferson introduces the concept of Global Virtual Time (GVT):

GVT at real time $r$ is the minimum of (1) all virtual times in all virtual clocks at time $r$, and (2) of the virtual send times of all messages that have been sent but have not yet been processed at time $r$.

Calculating the GVT is essential to any Time Warp based algorithm since it is necessary to efficiently collect the fossil objects for memory reuse. In a pure Time Warp environment, fossil objects are either states or events that are not going to be used anymore by the system. When a new GVT estimate is calculated. events and states whose timestamps are lower than GVT can be discarded.

A simple way to calculate the GIT is to use an synchronous algorithm in which processes involved in the simulation are halted and the system searches for the minimum message timestamp value. Once the calculation is over. processes are restarted. The main problem with this approach arises from the fact that synchronizing all processes is an expensive operation, especially if it has to be performed frequently. Hence, an asynchronous solution is generally preferred, but in this case, the real value of the GVT at time $r$ cannot be computed due to the intrinsic properties of distributed systems. Instead, an estimate of the GVT value is computed. This estimate gives a lower bound of the smallest timestamp of all the messages either in transit or waiting to be processed. Since no LP can rollback to a state prior the GVT. the system is free to discard all states older than the GVT as well as all the messages whose send time (for those stored in the output queues) or receive time (for those stored in the input queues) is lower than the GVT.

Most of the asynchronous GI’T algorithms [Prei89, Bell90, Lin90b, Conc91] are based on the same approach in which a token is passed around the processors and where the distributed computation is decomposed into two phases: a START phase and a STOP phase. 
Consider the case in which the processors are connected in a ring topology. In the first phase, a coordinator initiates the procedure and a $S T A R T$ token is passed around all the processors involved in the simulation. When a processor receives the START token from its predecessor, it forwards the token immediately to its successor and then starts to compute its Local Virtual Time, defined as being the minimum timestamp of all unprocessed events stored in the processor. In addition, as the simulation progresses, each processor keeps track of the smallest timestamp of all the messages it has sent since the $S T A R T$ token was received. If that timestamp happens to be smaller than the Local Virtual Time of the processor, then the LVT is set to that timestamp value.

The second phase is launched right after the coordinator has received the $S T A R T$ token back from its predecessor. At this time, the coordinator sends a STOP token containing its Local Virtual Time to its successor. When a processor receives the STOP token, it compares its Local Virtual Time with the GVT estimate included in the token. If the LVT happens to be smaller. the GVT estimate contained in the token is set to the LVT value handed in by the processor. The STOP token is then forwarded to the successor. When the coordinator receives the STOP token back from its predecessor, the value included in the token is the new GVT estimate which can be broadcasted to all the processors for fossil collection. The advantage of this algorithm is that the simulation is not halted during the computation of the GVT estimate. A two-phase algorithm is necessary in order to ensure that all the processors are together part of the computation at a given real time $t_{r}$.

Figure 1.4 gives an example of the GVT computation with 5 processors connected in a ring topology: Processor 1 initiates the computation at time $t=S T A R T_{1}$ and sends a $S T . A R T$ message to processor 2. Processor 2 receives the message at time $t=S T A R T_{2}$ and then sends another $S T A R T$ message to processor 3 . This procedure is repeated until processor 5 sends a $S T O P$ message to processor 1. During the real time interval $\left[S T A R T_{5}, S T O P_{1}\right]$ all processors are computing the GVT and the smallest timestamped message is inevitably found in that interval.

Another problem that must be considered is that of messages "hidden" in communication channels. Some parallel or distributed environments do not necessarily ensure atomic message delivery in which either the receiver or the sender always hold a copy of the message at any given 


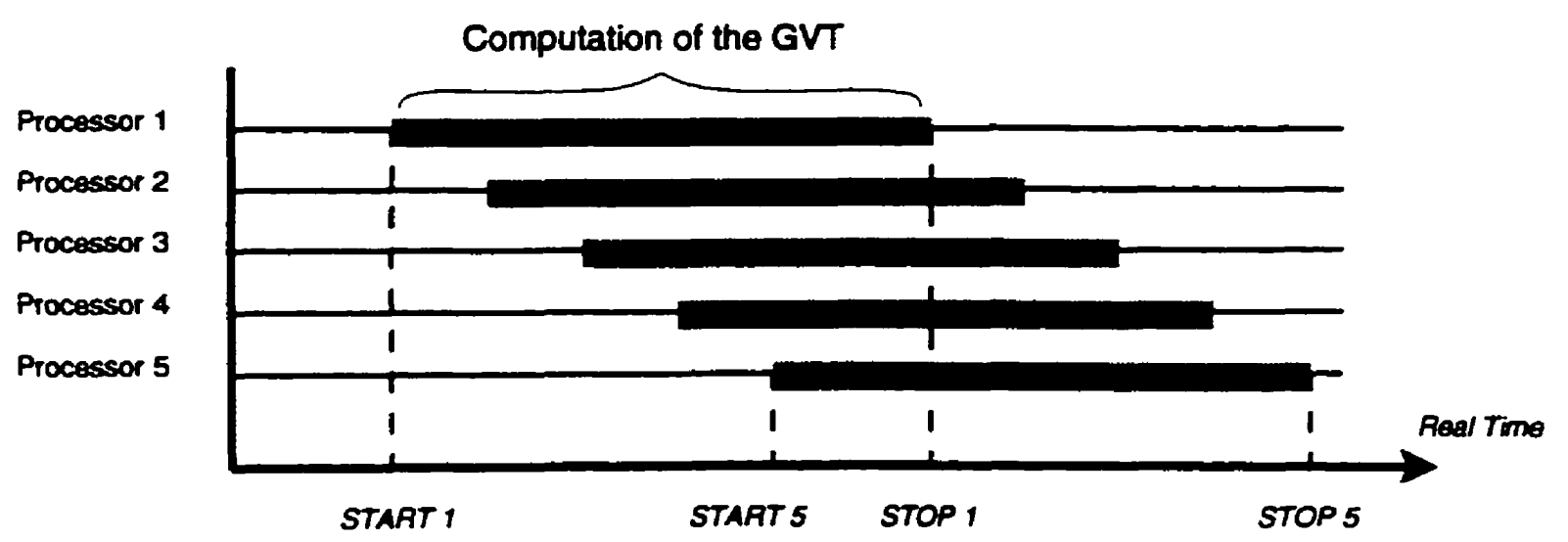

Figure 1.4: GVT Computation with 5 processors

time. In such cases. making sure that all processors are involved in the GVT calculation at a real time $r$ is not a sufficient condition to ensure the correctness of the computation. Bellenot [Bell90] tackles the problem by using acknowledgement messages. This is an expensive solution since it doubles the overall network traffic. Lin and Lazowska [Lin90b] attempt to reduce the communication overhead by acknowledging groups of messages when the GVT calculation is started. But when a fully connected network is used. their algorithm needs $n^{2}+2 n$ messages (where $n$ is the number of processes) in the worst case to calculate a new GVT estimate. The algorithms presented in [Bell90] and [Lin90b] are said to be active since they initiate the GVT. calculation by sending a $S T A R T$ message to the processors. Bauer and Sporrer [Baue92] propose a passive approach in which processors continuously calculate their LVT. Occasionally. they forward the relevant information to the GVT-calculation process on suitable occasions (along with simulation results for example). Their algorithm addresses the problem of network traffic effectively since less than $n$ messages on the average are needed to calculate a new GVT estimate where $n$ is the number of processors.

Concepcion and Kelly [Conc91] introduce the Multi-Level Token Passing (MLTP) algorithm based on the hypercube technology. Their principal motivation is to reduce the delay needed to calculate a new GVT estimate by parallelizing the computation. In their approach. a hierarchical structure is superimposed on the processors, thereby accelerating the computation. 


\section{Termination Detection}

Detecting the termination of the simulation is a straightforward procedure in Time Warp. Each time an LP runs out of input messages, it sets the value of its local virtual clock to $+\infty$. When the system terminates. there exist no message in transit, and all logical processes are idle. hence, having an LVT equal to $+\infty$. Therefore, when the GVT value reaches $+\infty$, termination is detected.

\subsubsection{Extensions to the basic Time Warp algorithm}

The main advantage of Time Warp is that it is deadlock-free since no process ever blocks if it has one or more messages in its input queue. On the other hand, Time Warp leaves many questions to be answered. First. it is an algorithm which can be greedy in terms of memory space. Second. it is unsure whether Time Warp has a stable behavior as there might be a danger of having cascading rollbacks. Lubachevsky. Schwartz and Weiss [Luba89] proved that a "chain reaction" of rollbacks may easily appear in an optimistic distributed simulation. To reduce the risk of cascading rollbacks. they suggest a method called filtered rollback which is based on the bounded lag algorithm developed originally by Lubachevsky [Luba88]. In their approach. a filter can be tuned so as to reduce the number of rollbacks by preventing events which are far in the future from being processed. hence making a simulation safer. In their analysis, the authors conclude by suggesting that an intelligent combination of conservative and optimistic techniques would be better than either approach in its pure form.

In [Stei92]. Steinman reports performance results of SPEEDES (Synchronous Parallel Environment for Emulation and Discrete Event Simulation), a parallel simulation environment developed at the Jet Propulsion Laboratory. In one of his experiments (a proximity detection simulation). Time Warp exhibits a startling rollback explosion effect. The number of messages and rollbacks increases exponentially crippling the simulation rapidly and making it impossible to complete. Steinman ran exactly the same simulation using the Breathing Time Bucket [Stei91] algorithm and no such explosion was observed since antimessages are not required in this approach. 


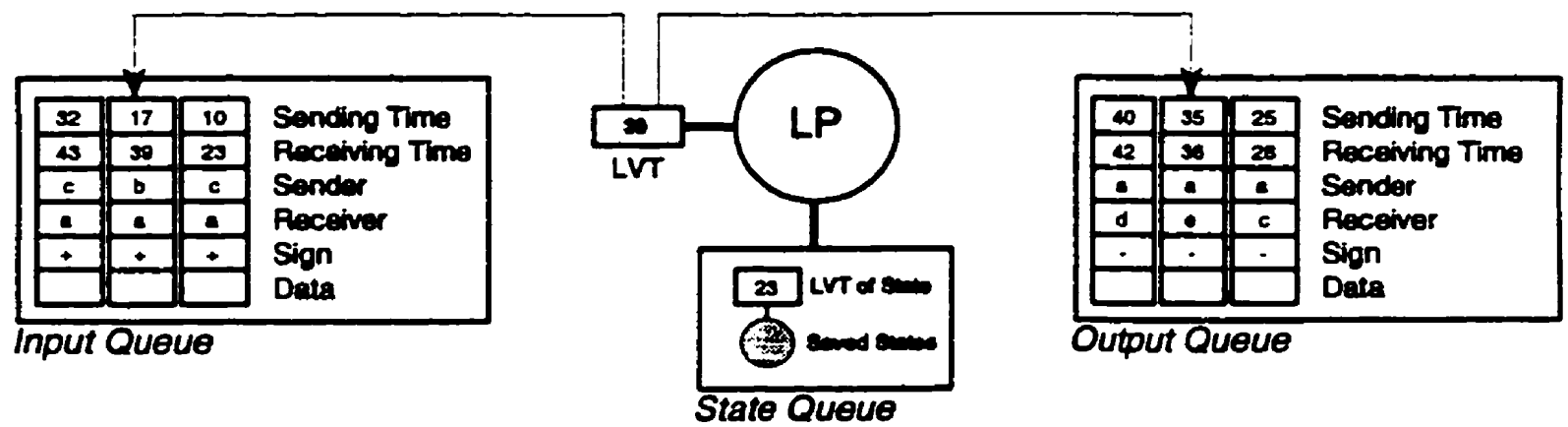

Figure 1.5: Time Warp Environment of the LP after message at 34 was cancelled and if lazy cancellation is used

Good performance of the Time Warp mechanism depends on the gamble that rollback does not occur frequently enough to become a significant overhead to the system. Jefferson [Jeff85] claims:

$\ldots$ in a realistic system rollback is not as costly on the average as one might fear.

We conjecture that most programs obey a temporal locality principle.

Jefferson also makes an interesting analogy between the Time Warp mechanism and virtual memory by comparing a rollback to a miss. We proceed to examine some of the approaches developed in an attempt to address these issues.

\section{Lazy Cancellation}

Different strategies have been proposed to reduce the number of rollbacks. Gafni [Gafn85] proposes the use of lazy cancellation instead of aggressive cancellation. Under lazy cancellation. antimessages are not immediately sent after a rollback. It is suggested that it is likely that only a small part of the computation is wrong due to the arrival of a straggler. Therefore, the LP keeps processing messages and checks if any output message matches with one which has been already transmitted. If that is the case. the antimessage is not sent and the output message is simply discarded. If the output message is different from one which has already been transmitted, the necessary antimessages are sent in addition to the output message.

Starting from the example shown previously in figure 1.3. figure 1.5 shows the state of the system after the message at 34 was cancelled assuming that lazy cancellation is used. Notice 
that antimessages are not sent immediately. Instead, they are kept in the output queue.

Berry [Berr86] points out that lazy cancellation has a chance to perform better than aggressive cancellation because an event might be correct, even though it has been sent for the wrong reasons. Gafni [Gafn88] also suggested that lazy cancellation can achieve faster execution time because of the super-criticality [Srin95, Berr85] characteristic of Time Warp. A protocol is said to be super-critical if there exists at least a simulation which can complete in less than the critical path of the simulation. In the case of lazy cancellation, a process may correctly guess the dependence of some events prior to their arrival and may not need to rollback its successors. Nevertheless, Briner's empirical results [Brin90] do not correlate with Berry's premises. In the domain of well balanced optimistic logic simulations, he observed that no substantial improvement is obtained from choosing lazy cancellation over aggressive cancellation because all processors tend to be in the same timeframe. In some cases, lazy cancellation performed even slightly worse than aggressive cancellation due to the overhead incurred by the technique. Briner's results are confirmed by the performance study conducted by Reiher, Fujimoto. Bellenot. and Jefferson [Reih90] in which very little difference was observed between lazy and aggressive cancellation. Depending on the particular application, either one of the two algorithms performed slightly better. Typically, less than a $10 \%$ difference in simulation time was observed.

Furthermore, it is unsure whether lazy cancellation might, in fact, create other problems. For example, since it takes more time for the system to cancel wrong computation, the performance of the system might not be as good as under the aggressive cancellation strategy. This problem is characterized by Fujimoto [Fuji89] as "the dog chasing its tail" effect in which the system is not able to cancel erroneous computations faster than they propagate.

\section{The Rollback Chip}

Fujimoto. Tsia. and Gopalakrishnan [Fuji88] propose to use a roll back chip whose only purpose is to take care of the state saving and the state management, thus freeing the main processor of excessive overhead. Buzzell, Robb and Fujimoto [Buzz90] give the first results obtained from adding the special rollback chip to a Butterfly multiprocessor machine. Results show a dramatic improvement in the performance, especially when the state size of the logical processes is 
rather large (1 Mbyte). The authors claim that the special rollback hardware can actually be economically produced.

\section{Checkpoint Interval}

One of the main disadvantages of the Time Warp approach is the amount of memory usage. This can be significant, especially if the size of the simulation system and/or the size of the state of the processes is large. Logical processes have to make checkpoints periodically in order to be able to roll back to an earlier state in the case a synchronization error occurs. In the Time Warp mechanism initially introduced by Jefferson [Jeff85], LPs checkpoint their state before processing each incoming event. One way to reduce the memory consumption is to change that checkpoint interval and to make it larger. When a rollback occurs. the LP restores the state whose checkpoint is prior to the receive time of the straggler. Then the LP rebuilds its state by reprocessing all of the input messages whose receive time is prior to that of the straggler. This last operation is called coast-forward.

Preiss, MacIntyre and Loucks [Prei92] examine the relationship between time and space in optimistic techniques using different checkpoint intervals (from 1 to 15 ) in their implementation of Time Warp. Their data show that the maximum total storage needed with a checkpoint interval of 1 can be reduced by $30 \%$ with a checkpoint interval of 2 and halved with an interval of 5 . With checkpoint intervals of 6 and up. no further substantial memory saving is observed. These results were obtained when the state size of an LP was 664 bytes. Further. the authors observe that the time-optimal and space-optimal checkpoint intervals are not necessarily identical.

Lin. Preiss. Loucks and Lazowska define a checkpointing model in [Lin94] and show that in general. a checkpoint interval of 3 saves a substantial amount of memory without greatly affecting the performance of the system.

Different methods have been developed in an attempt to find the optimal checkpointing interval. Lin [Lin92b] develops upper and lower bounds for checkpoint intervals. Experimental results showed that the optimal checkpoint interval is actually closer to the upper bound. Rönngren and Ayani [Ronn94] present an adaptive checkpointing algorithm based on the use of exponential smoothing. Their results show that a substantial amount of memory is saved. Nevertheless, 
since their simulation model is small (less than a hundred logical processes), it is difficult to extrapolate these results to large models.

\section{Flow Control}

Under Time Warp, flow control is a critical and difficult issue since the system must deal with incoming and outgoing messages and keep copies of these messages as well as copies of the states of the processes. One of the first mechanisms to deal with the flow control issue is the message sendback protocol, proposed by Gafni [Gafn85]. In his approach. when a process runs out of memory, it simply sends back input messages to their senders, which roll back and end up retransmitting the same messages again until the receiver has finally enough memory space to buffer them. Since a process can only delete its own items, Gafni's protocol may still consume unbounded memory. In [Jeff90]. Jefferson proposes the cancelback protocol in which a process that requests storage has the ability to cancel items in other processes. One of the problems of the message sendback and cancelback protocols is known as busy cancelback, in which a process [Jeff90] keeps being rolled back and resending messages to one of its successors which continuously lacks memory space because of its own computation. Furthermore, Lin and Preiss show in their study [Lin91b] that there exists a possibility for both protocols to enter into an infinite loop. To solve these problems. the authors proposed the artificial rollback algorithm, in which a process may roll itself back if more memory is needed. The authors show that if the artificial rollback protocol is executed frequently enough. the space complexuty of a Time Warp simulation is the same as that of a sequential simulation.

Das and Fujimoto [Das93] experimentally evaluate the effect of the cancelback protocol under various overheads on a 32 processor shared memory machine. They observed that the use of the protocol may significantly improve the performance of the system. Their data also show that the cancelback protocol allows a limited memory simulation to be at most $10 \%$ slower than an unlimited memory simulation. In [Lin92], Lin evaluates the three algorithms and finds that both cancelback and artificial rollback protocols are optimal for a shared-memory architecture, which might explain in some measure the good results obtained by Das and Fujimoto. 


\section{Variants of Time Warp}

Sokol, Briscoe and Wieland [Soko88] proposed the Moving Time Window approach which prevents the logical processes from going too far ahead in time, therefore limiting the potential risk of getting rollbacks.

Steinman [Stei93] presents a variant of Time Warp called Breathing Time Warp which has been implemented in the SPEEDES operating system [Stei91]. The major motivation of Steinman's approach is to reduce the risk so that an antimessage explosion ever happens. Breathing Time Warp is a mixture of Time Warp and the Breathing Time Buckets approach. which is an optimistic windowing approach being "conservative enough" so that antimessages are not needed [Stei93]. Breathing Time Warp basically switches dynamically between pure Time Warp. and the Breathing Time Buckets mechanism. In his test results, Steinman points out that Time Warp can exhibit rollback explosions whereas Breathing Time Warp was rather stable. He also claims his new approach performs slightly better than Time Warp (even though performance results were not given).

In [Raja93], the authors present the Local Time Warp approach. that combines both a conservative and an optimistic algorithm. Logical processes are grouped into clusters. Time Warp is used within the clusters and a conservative time window algorithm is used between clusters. This approach aimed at reducing the risk of cascading rollbacks. sensitivity to lookahead. and the scalability problems. Despite the fact that a prototype of the Local Time Warp algorithm was operational, no performance results were given.

\section{Performance}

Fujimoto [Fuji90] reports performance results of a Time Warp implementation running simulations of closed queueing networks on a BBN Butterfly Plus (a virtually-shared memory machine) and on a Hypercube (a distributed memory machine). The results show that "speedup does scale as the problem and machine size are increased". and a speedup of 56.8 using 64 processors was even observed for one of the benchmarks.

Madisetti, Walrand and Messerschmitt [Madi90] analyzed different optimistic algorithms. The authors find that the progress of the computation not only depends on the rate of the slowest 
processor but also on the interaction between the slowest and the fastest processors. The authors also introduce a new model of distributed computation called the self-synchronizing concurrent computing system (SESYCCS) in which they propose that the synchronization should be enforced in the kernel by the system itself instead of being part of the computation. The MIMDIX Operating System provides synchronization facilities at the kernel level and performance results are given in [Madi92]. The authors compared the MIMDIX Operating System to the first generation of Time Warp systems by running a simulation model of a large distributed electric power distribution grid on the 32-processor BBN Butterfly. Results showed that MIMDIX easily outperformed Time-Warp by a factor of about four.

\subsubsection{Hybrid approaches}

\section{ADAPT}

Jha and Bagrodia describe in [Jha94] a parallel simulation protocol called $A D A P T$ in which logical processes may choose dynamically to use either a conservative or an optimistic approach. Some systems can be composed of several subcomponents characterized by different properties. making it difficult (or even impossible) to choose a single synchronization algorithm suitable for all the components. Properties such as the event granularity, the amount of available memory or the asymmetry of the workload may affect the performance of the system depending on the. synchronization mechanism being used. The $A D A P T$ protocol attempts to solve this problem by letting the submodel choose dynamically between a conservative or an optimistic approach. The main difficulty of this protocol is to carefully choose a set of heuristics on which to base the decision of switching from one mode to another. Clearly, such heuristics depend mostly on the characteristics of the model. The authors present several interesting and intuitive ideas to solve this problem but no experiment results are given.

\section{Space-Time}

Chandy and Sherman [Chan89] propose a unifying framework called space-time. They represent the behavior of the system by a two dimensional diagram in which the $\mathrm{x}$-axis is space and the $\mathrm{y}$ axis is time. Uising this framework, they study the behavior of the different simulation techniques 
such as time-driven simulation, sequential discrete-event simulation. conservative distributed simulation, and optimistic distributed simulation. They also present a new simulation algorithm in which space-time regions are treated as static-objects. Each region is assigned to a processor which computes its behavior using estimated states of its neighboring regions and informs them of the changes regarding the behavior of the region. The system iterates until no change in the estimates is observed. The algorithm then converges to a unique solution and terminates when a fixed point is reached.

Bagrodia. Chandy and Liao [Bagr91] present an experimental study of the performance of space-time in which the authors study three types of queueing networks: a feed-forward network. a closed queueing network. and a variation of a closed queueing network in which jobs are assigned different priorities. Speedups of up to 55 with 64 processors were observed. Nevertheless. the data showed that the performance of the simulation depends highly on the size of the iteration step. For example. by halving the iteration step, rollbacks were increased by more than 8 times. reducing the speedup by $60 \%$. It is still very unclear how to accurately choose the iteration step since it depends highly on the type and behavior of the simulation model.

\subsubsection{Comparing Time-Warp and the Chandy-Misra approach}

Lin and Lazowska [Lin90] compared both the conservative and the optimistic approaches. They first show that Time Warp with aggressive cancellation is not in general conservative optimal. A conservative optimal simulation gives a lower bound on the execution time for conservative parallel simulations. This is because optimistic systems experience rollbacks and the number of messages generated tends to be larger than in a conservative optimal simulation. They also formally demonstrate that the Time Warp approach outperforms the Chandy-Misra approach in every feedforward network simulation (feedforward graphs are directed acyclic graphs or dag). They consider the Chandy-Misra approach with null messages for deadlock avoidance. Assuming that the overhead of handling these null messages is nil, Time Warp still outperforms the conservation approach. In addition, Lin and Lazowska show that for feedback networks without lookahead, Time Warp outperforms the Chandy-Misra approach in most cases. Nevertheless. these conclusions are based on the assumption that memory is unbounded. 
Lipton and Mizell [Lipt90] give a worst-case comparison between Time Warp and the ChandyMisra algorithm. Under their simplifying assumptions, they show that there exists a simulation model on which Time Warp outperforms the Chandy-Misra algorithm by a factor of $n$, where $n$ is the number of processes in the simulation. They also show that the Chandy-Misra approach can only outperform Time Warp by a constant factor.

Simplifying assumptions are necessary to analytically describe a model and draw general conclusions from it. However, the behavior of distributed simulation algorithms is very complex and assumptions such as unbounded memory and a negligible state saving overhead make such analysis meaningless. On the other hand. analyzing the behavior of distributed simulation algorithms with realistic assumptions is difficult. In addition. systems composed of a large number of agents interacting with each other have a behavior which is not easily explicable, even if the behavior and the local interactions of each individual agents is well understood. Such systems are said to be emergent [Hube93. Glan95. Lux95] and their higher-level properties cannot be easily explained or deduced by the properties of their lower-level entities.

\subsection{Logic Simulation and Parallel Discrete Event Simu- lation}

There are two traditional algorithms for logic simulation on parallel machines. One is referred to as a compiled-mode simulation in which all elements of a circuit are evaluated on each clock tick. The other approach is the centralized-time, event-driven approach in which only the elements whose input has changed are evaluated on each clock tick. This method eliminates the redundant work performed by the compiled-mode method. however. the amount of parallelism achievable is limited due to the fact that too few gates are active at any instant.

Serious interest in Parallel Discrete Event Simulation algorithms started to arise in the 1980s and publications flourished. Asynchronous algorithms such as the ones we described above. either using a conservative or an optimistic approach were thought to achieve better performance. Soule and Gupta [Soul91] show that for 2 benchmark circuits, the Chandy-Misra algorithm can extract between 40 and $107 \%$ more concurrency than traditional approaches such as time-stepped techniques. 
In [Bail88] Bailey and Snyder exhibit some pessimism. On a set of benchmark circuits, they did not find a great potential for parallelism. Actually they find it "remarkably small" and illustrate their claim with an example. They chose the Quarter Horse, a 32-bit RISC microprocessor containing about 24,000 transistors.

For instance, for the Quarter Horse if we used 139 processors, and there was no overhead for synchronization and communication, the speedup would be only 6.3 .

However, their work focused only on the number of simultaneous events occurring at the same virtual time which is only meaningful in the simulation system using a time-stepped technique. In a time-stepped simulator, the simulation time scale is divided into small regions, and the model is evaluated for each of these regions, one after the other, and not concurrently. In a simulator using an asynchronous algorithm, events are evaluated regardless of their time region, all that matters is that causally related events are processed in the correct order. The great advantage of this method is that the simulation progresses at a faster pace because the system can concurrently. process messages located in different time regions without violating the causality principle which preserves the correctness of the system.

When using the Chandy-Misra algorithm for digital logic simulation with the deadlock detection and breaking approach. Soule and Gupta [Soul89] find that between $40 \%$ and $60 \%$ of the total execution time is spent on deadlock detection and resolution. To solve this problem. they identify four types of deadlocks: register clocks and generator nodes. multiple paths, unevaluated paths, and the order of node updates. The final result of their study show that the CMB algorithm can outperform the centralized-time, event-driven algorithm by a factor of 2 to 15 using up to 64 processors on an ideal multiprocessor by a factor of 7 to 9 on a 16-processor Encore Multimax.

M.J. Chung and Y. Chung present [Chun90] results of a digital simulator based on the Time Warp approach for the Connection Machine. Because of the lack of a flow control mechanism in their system, they found that too many events are generated causing storage overhead. To solve this problem, they designed the Lower Bound Rollback method that is based on the characteristics of the circuit graph dependency. By partitioning the circuit into acyclic subgraphs, it then 
becomes possible to determine the lower bound of rollback (LBR) time for each node. Therefore, all the events up to the LBR time can be discarded, hence reducing the memory usage.

Bailey and Lin [Bail92] analytically compare the synchronous protocol, the Chandy-Misra protocol, and the Time Warp protocol with aggressive and lazy cancellation. The authors use an artificial protocol called the conservative optimal protocol as a basis for their comparison. They show that the Chandy-Misra-Bryant approach cannot achieve the performance of the conservative optimal protocol whereas Time Warp outperforms it, whether lazy or aggressive cancellation is used. However, the problem of memory management and communication overhead were not addressed.

\subsection{Scope of our research}

While research has been undertaken to find out whether Parallel Discrete Event Simulation techniques can be successfully applied to Logic Simulation. it is still uncertain as to whether the distributed synchronization algorithms developed so far can serve as a basis for building a parallel digital logic simulator.

While parallel discrete event simulation has raised a lot of interest for the past decade, no algorithm stands out as a general solution to the synchronization problem. The performance of asynchronous algorithms we have described above is strongly tied to the type of model being simulated. Some conservative techniques may perform better than optimistic techniques in very specific cases. The opposite also holds. Therefore, most of the comparison studies whose goal is to define what approach is best may not apply to real-world problems since they are based on benchmark simulation models such as the Shark's World [Conk90. Pres90. Bagr91] or the Colliding Pucks models [Hont89].

Techniques for distributed simulation should not be seen as a product which solves general problems, but rather as a service. whose goal is to solve a very specific problem. Therefore, rather than starting from a solution and applying it to a simulation model. we will start to analyze the model itself, determine its characteristics and its behavior. and then try to find the best possible solution.

This research focuses on the possibility and feasibility of using parallel discrete-event simulation 
techniques to run logic-level simulations of medium or large sequential circuits on general-purpose distributed memory (ie: message-passing) architectures. A road-map to the contents of the thesis follows.

In chapter 2, we analyze the characteristics of logic circuit simulation such as circuit parallelism, circuit design, workload distribution and workload variability.

In chapter 3, we describe a novel approach, Clustered Time-Warp (CTW), to parallel discreteevent simulation which is based on Time-Warp, and which takes advantage of the characteristics of digital circuits. We also introduce a family of three different checkpointing techniques, each of which occupies a different point in the spectrum of possible trade-offs between memory usage and execution time.

The Clustered Time Warp algorithm and the checkpointing techniques were implemented in a digital logic simulator and its performance compared to that of Time Warp. Results are presented and discussed in chapter 4.

In chapter 5. we describe a dynamic load-balancing algorithm developed for Clustered TimeWarp which takes advantage of its clustering approach. We measure the effects of the algorithm on workload distribution . inter-processor communication and rollbacks, and compare its performance to that of Time Warp.

In chapter 6 , we study the effects of different circuit partitioning and mapping strategies on the performance of the Clustered Time Warp algorithm.

In chapter $\bar{l}$, we investigate the scalability of both the Time Warp and the Clustered Time Warp approach. Simulation of large queueing network models are conducted and the performance results are reported.

Finally, chapter 8 gives a summary of the thesis and suggests future extensions to our work. 


\section{Chapter 2}

\section{On the Characteristics of Logic Circuit Simulation}

\subsection{The Simulation Model}

Logic circuit models are composed of two distinct entities: behavior and signals. Behaviors are routines representing logic gates, and signals correspond to interconnections between behaviors. the wires of the physical system.

The basic component of a circuit is the logic gate. Its behavior is known and corresponds to a boolean function. NAND. AND, NOR, OR are examples of typical functions provided by a gate-level simulator. Gates are interconnected forming a directed graph which is directly mapped onto the LPs. Incoming vertices of an LP correspond to the inputs of the logic gate. Similarly, outgoing vertices correspond to the outputs of the gate.

The model uses a finite set of states to represent the type of signal propagating throughout the circuit. Like most simulators, the system must be able to use different state values such as HIGH. LOW and X (undefined). THOR [Smit87] and LDVSIM [Brin90] provide this level of modelling. However. modern simulators use other values to represent floating signals or high-impedance conditions. The IEEE standard logic system for VHDL simulation [Bill93] uses 9 logic values (table 2.1).

A signal change is modelled as an event (or message) which basically contains the time at which the signal change occurred. an identification of the node from which the message was originated, and a value which corresponds to the new state of the line. When an LP receives a 


\begin{tabular}{|r|l|}
\hline State & Purpose \\
\hline U & Uninitialized state \\
$\mathrm{X}$ & Forcing unknown \\
0 & Forcing zero \\
1 & Forcing one \\
$\mathrm{Z}$ & High impedance \\
$\mathrm{W}$ & Weak unknown \\
$\mathrm{L}$ & Weak zero \\
$\mathrm{H}$ & Weak one \\
- & Don't care \\
\hline
\end{tabular}

Table 2.1: IEEE Standard Logic System

message. it sets its local clock to the value of the message time, and then reevaluates the output of the gate which it models taking into consideration the change that occurred. If the output happens to be different after the reevaluation. the LP will send a message on its outgoing links to notify its successors of the signal change. The time of this outgoing message is the time of its local clock plus a service time which approximates the delay of the gate (ie: for the signals to propagate through the transistors of the gate). Because timing is not our main concern here. such an approximation is accepted in order to quicken the simulation process.

\subsection{Discrete Event Simulation}

Figure 2.1 represents a simple logic circuit made of three gates. The circuit has three inputs ( $a$. $b$ and $c$ ), one output $(f)$ and two internal nodes ( $d$ and $e$ ). The diagram on the right of the figure shows the state of the nodes of the circuit with respect to real time. For example. the input $a$ rises from LOW to $\mathrm{HIGH}$ at real time $t_{r}=10 \mathrm{~ns}$. This causes the OR gate to reevaluate its output $d$. If we assume that the delay for a signal to propagate through a gate is $2 n s$, the signal at node $d$ rises from $\mathrm{LOW}^{*}$ to $\mathrm{HIGH}$ at real time $t_{r}=12 n s$. This change triggers the AND gate to be reevaluated, and the output $f$ to rise at $t_{r}=14 n s$. The change of state at node $c$ at $t_{r}=20 n s$ also propagates through the NOT gate and the AND gate, causing the output $f$ to fall at $t_{\mathrm{r}}=24 n s$. 

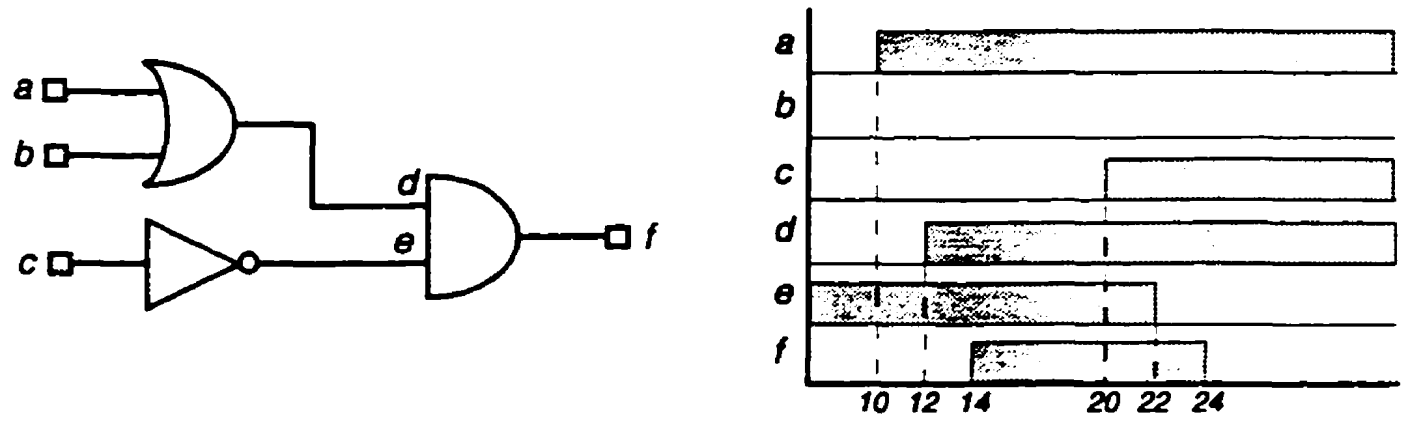

Real Time (ns)

Figure 2.1: Example of a small circuit

In a discrete event simulation, each gate is modelled as a Logical Process. wires are communication channels between processes and an event queue is associated with each input port. If we were to simulate the above example. the model would start with two input events. each of them representing a change of state in one of the input ports. An event contains two types of information: the new state of the node and the time at which the change occurs. In addition. each process has a local clock (Local Simulation Time or LST) initialized to 0. Figure 2.2 shows the initial state of the simulation.

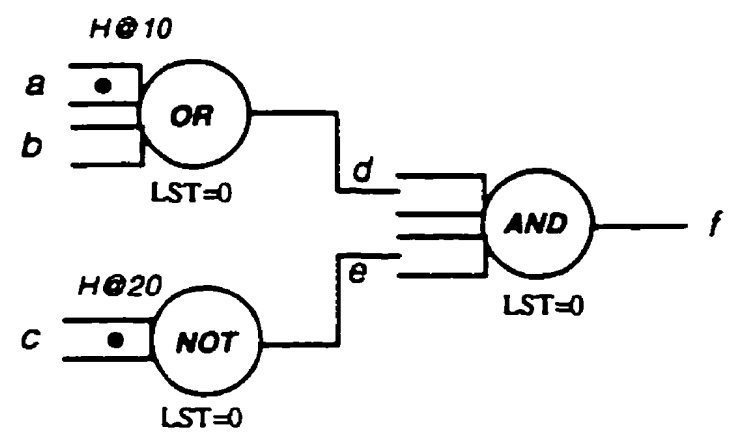

Figure 2.2: Initial state of the discrete event simulation

The basic discrete event simulation algorithm can be described as follows:

1. The event $e$ with the lowest timestamp value $t(e)$ is chosen.

2. The receiving process $L P_{\imath}$ updates its Local Simulation Time: $L S T_{L P_{1}} \leftarrow \min \left(L S T_{L P_{1}}, t(e)\right)$.

3. Process $L P_{i}$ reevaluates its output. 
4. The service time $\tau$ is added to the $L S T$ of $L P_{i}: L S T_{L P_{i}} \leftarrow L S T_{L P_{i}}+\tau$.

5. If the output of $L P_{i}$ has changed, an event $e^{\prime}$ with timestamp $t\left(e^{\prime}\right)=L S T_{L P_{i}}$ is created and sent to the successors of $L P_{i}$.

This algorithm is repeated until there is no more event to process. Figures 2.3 and 2.4 show the four successive states of the system as the simulation of our circuit progresses.
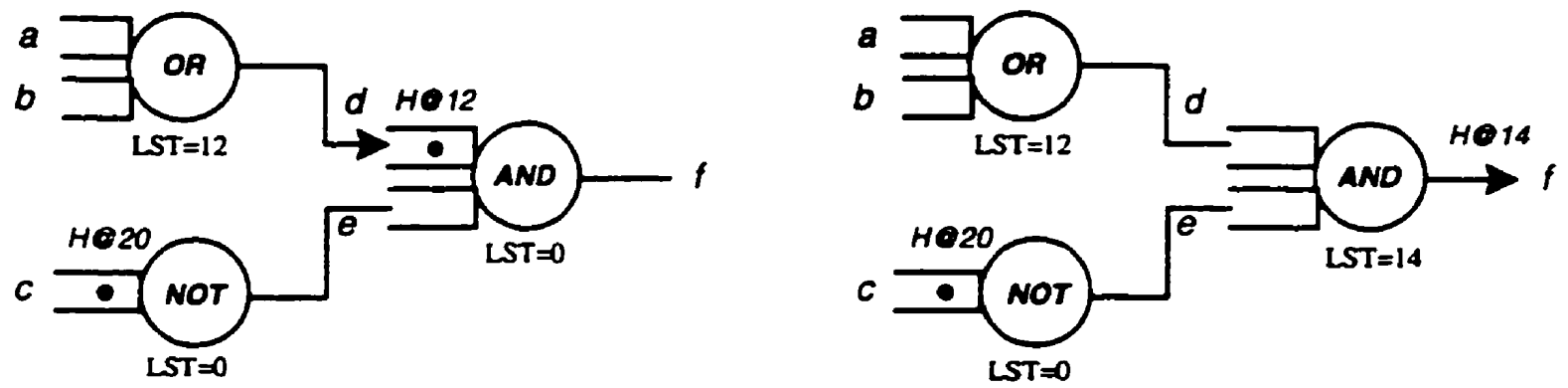

Figure 2.3: The simulation system at $t_{s}=12$ and $t_{s}=14$ respectively

The process modelling the OR gate is first triggered since it holds the event with the smallest timestamp value. Its simulation time is set to $10 \mathrm{~ns}$. the event is processed. and the service time of $2 \mathrm{~ns}$ is added to the process LST. Since the output of the LP has changed, an event with timestamp 12 is sent to the process which models the A.ND gate. The system is now at simulated time $t_{s}=12$ and its state is depicted by the left hand side diagram of figure 2.3. The AND process is now triggered. Its event is dequeued. its LST is updated to $12 \mathrm{~ns}$, and its state is reevaluated. The service time is added to the process simulation time. and an event timestamped with the new LST value is sent to model the change at the output of the gate. The system is now in its second stage at simulated time $t_{s}=14$ (right hand side diagram of figure 2.3).

The LP modellirg the NOT gate can now be unblocked (figure 2.4). Its LST is set to 20ns, the event is processed, a service time of $2 \mathrm{~ns}$ is taken into consideration, and an output event with timestamp 22 is sent to the successor. Finally, the last event is processed and the simulation stops at time $t_{s}=24$.

For the sake of clarity, we make use in our example of a unit-delay to model the time needed for the signal to propagate through the gate. Unit-delay is used when timing accuracy is not 

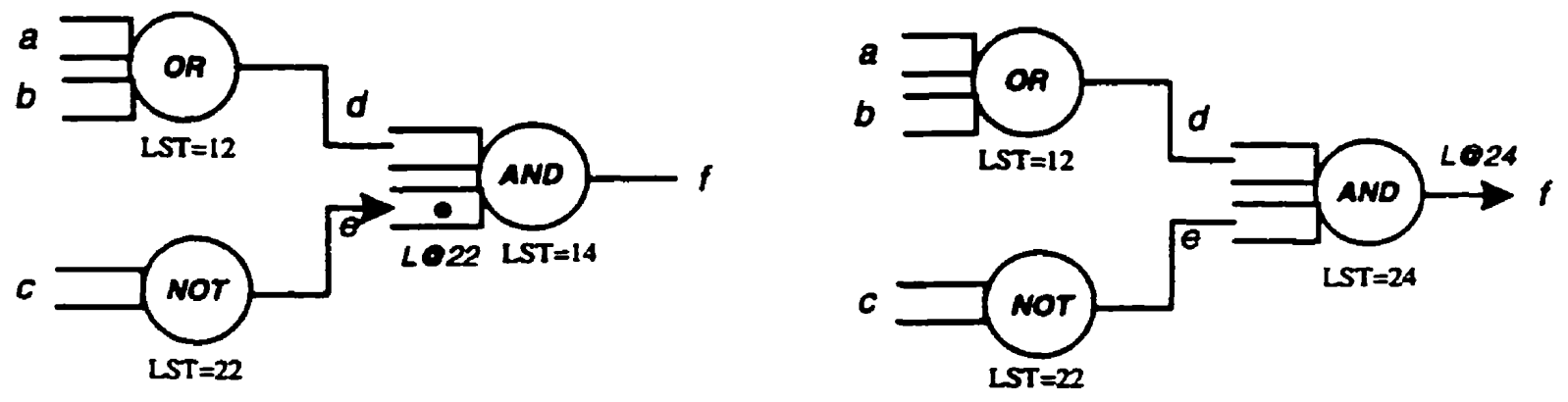

Figure 2.4: The simulation system at $t_{s}=22$ and $t_{s}=24$ respectively

really needed and when simulation time is more critical. SwitchSim [Fran85] is an example of a unit-delay switch-level simulator. When accurate timing analysis is necessary each process can be associated to a particular service time representing the delay of the gate it models. For example. RNL [Term83] is a variable-delay switch-level simulator.

In some cases, distribution functions are used to determine at runtime the service time of the processes. This approach is used in application domains such as queueing networks [Bouk95b]. Such service time distributions include the uniform distribution. the exponential distribution or the binomial distribution [Bouk95].

\subsection{Circuit Design}

A typical planning process to design a digital circuit is decomposed into three phases [Zimm86]:

- The behavioral design in which the system specification are developed.

- The structural design which transforms the specifications into a structural description (logic synthesis).

- The physical design phase during which the physical description of the circuit is elaborated.

Due to the increasing complexity of VLSI circuits, designers use a hierarchical methodology to develop their systems in order to simplify the design process. A circuit is first decomposed into a set of functional units which are interconnected. For example, the Texas Instrument's microSparc, produced in 1993, includes a 32-bit integer unit, an FPC, a Memory Management 
Unit, a data aligner/parity checker, a DRAM controller, an I/O bus controller, an instruction cache. and a data cache. Similarly, each of these functional units is decomposed into blocks depending on their relative complexity. The depth of the hierarchical structure depends on the complexity of the circuit. Another example of the hierarchical approach used by circuit designers is the Intel's sixth-generation P6 processor depicted in figure 2.5 [Byte95].

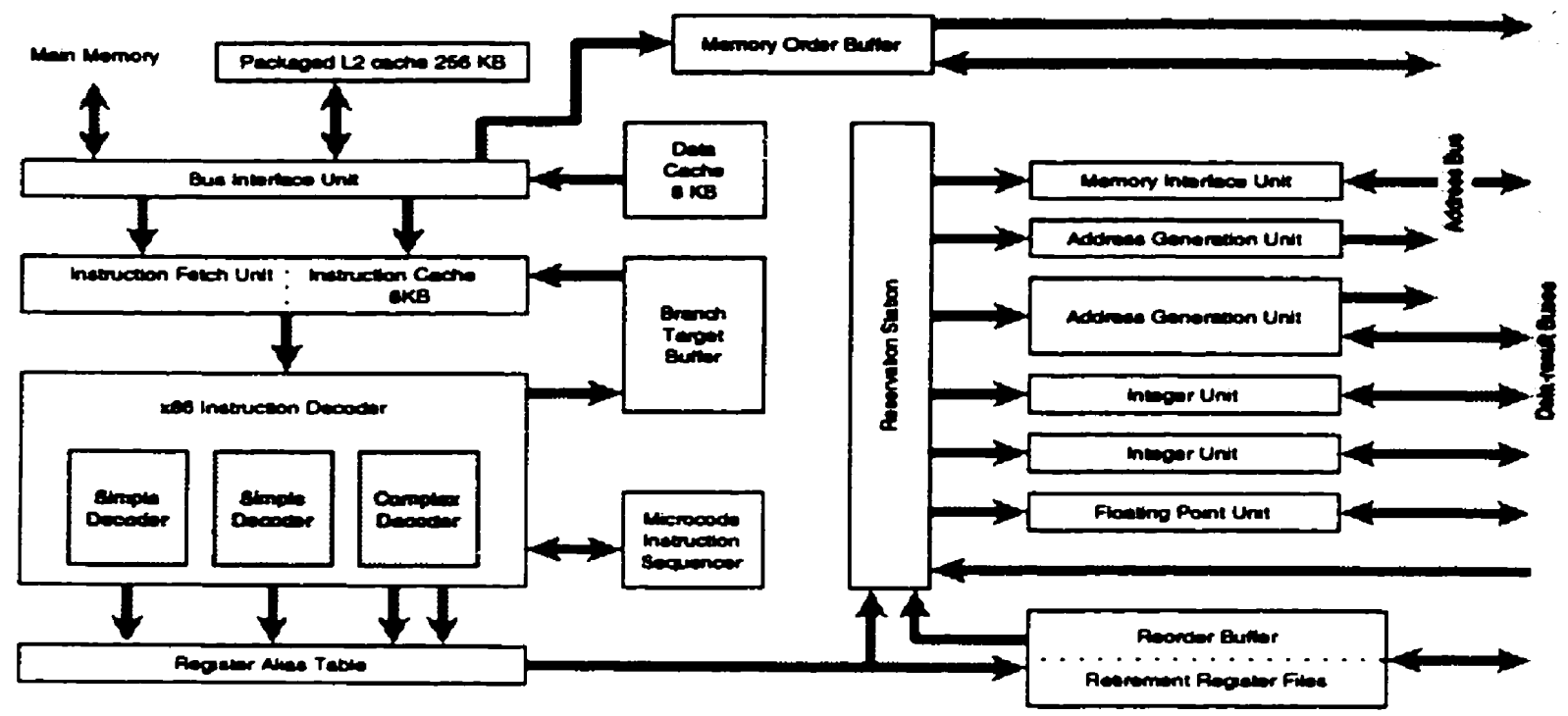

Figure 2.5: Building blocks of the Intel P6

\subsection{Circuit Parallelism}

In this section. we study the inherent parallelism existing in digital circuits. Such information is fundamental for determining the upper bound of the speedup achievable by an asynchronous simulation algorithm.

\subsubsection{Related Results}

There have been some pessimistic results regarding the inherent parallelism arailable in digital circuits. Mary Bailey [Bail92b] shows that the inherent parallelism of VLSI circuits is very low, and does not increase with size. In her study, the parallelism of nine circuits ranging in size from 200 to 61.600 transistors was analyzed with two switch-level simulators: RNL [Term83] and 
SwitchSim [Fran85]. Two simulation metrics were used: the event metric which is the average number of events executed during time steps in which at least an event occurred, and the queue metric which is the average queue length during a simulation step. The event metric gives an upper bound of the speedup achievable by a synchronous parallel event-driven simulator. while the queue metric approximates the number of nodes in the circuit which are changing simultaneously. Given the simulation results, Bailey measured the average and the relative parallelism of the circuit. The average parallelism is the average number of concurrent events during the simulation run. The relative parallelism is defined as being the average parallelism divided by the number of transistors in the circuit. Results show that the relative parallelism vary widely for different circuits. In one case, the relative parallelism of a circuit outperformed by a factor of 43 that of another circuit. This type of variation is understandable since the activity of a circuit is most of all related to its functional characteristics rather than its size. For example. some circuits can be pipelined which is likely to increase the parallelism of the circuit. On the other hand. Programmable Logic Arrays ( $P L A s)$ are likely to have a small parallelism.

Frank [Fran85] analyzed 13 circuits ranging in size from 78 to 20.300 transistors and found that the potential speedups were surprisingly low, ranging from 4 to 192 with a mean of 50 .

In the studies described above [Fran85. Fran86. Bail92b], the metric used to measure the upper bound of the potential speedup of parallel simulators is usually referred to as circuit parallelism. This metric is defined to be the average number of events executed per simulation step where the simulation step is a simulated clock cycle of the circuit. Such a metric can only apply to event-driven synchronous simulators in which processors work on the same time step in a synchronous lock-step fashion. In the case of asynchronous algorithms. many processors can work on different parts of the circuit in different time regions while the correctness of the simulation is still preserved. For example, events which happen at the same simulated time, can be executed by one or more processors at different moments during the simulation. Similarly, events which happen at different simulated time can be executed simultaneously by several processors. Therefore the upper-bound of the speedup achievable by an asynchronous simulator can only be higher (or equal in the worst case) to that of a synchronous simulator. 


\subsubsection{Critical path analysis}

In a single processor environment, only one event at a time can be processed. One of the advantages of distributed simulation is to extract the parallelism available in the simulation model and to process events concurrently on several processes. Nevertheless, the completion time of a distributed simulation is limited by the critical path of the event dependence graph. Berry and Jefferson introduce in [Berr85] the Conservative Lower Bound (CLB) which is the potential parallelism measured from the analysis of the event dependence graph. All evaluation times are constant and the communication overhead is assumed to be nil. In [Brin89, Brin90], Briner extends the algorithm to take into consideration different model evaluation times and communication delays. In his study, the author estimates the potential parallelism of a circuit after having analyzed the causal relationships between events. Experiments on circuits ranging in size from 700 to 15,000 transistors show that the circuit parallelism measured by the spanning metric is actually 4 to 10 times higher than that measured by simpler metrics used to measure the upper bound of the potential speedup achievable by synchronous algorithms.

A simulation run can be represented by a space-time diagram such as the one depicted in figure 2.6 where the space coordinates ( $y$-axis) represent the processes at which events are executed and the time coordinates ( $x$-axis) represent the simulation time.

This space-time representation is based on the message-initiating model which defines the four following relations on events:

1. Event $e$ is a predecessor of event $e^{\prime}$ (or $e^{\prime}$ is the successor of $e$ ) if the three following conditions are satisfied:

- $e$ and $e^{\prime}$ are executed by the same process $L P_{i}$.

- $t(e)<t\left(e^{\prime}\right)$ where $t(e)$ and $t\left(e^{\prime}\right)$ are the timestamps of events $e$ and $e^{\prime}$ respectively.

- $\nexists e^{\prime \prime}$ such that $t(e)<t\left(e^{\prime \prime}\right)<t\left(e^{\prime}\right)$.

The predecessor of event $e$ is denoted pred $(e)$.

2. Event $e$ is the antecedent of event $e^{\prime}$ if the execution of $e$ sends a message which schedules event $e^{\prime}$. The antecedent of event $e$ is denoted ante(e). 
3. $e$ is said to immediately affects $e^{\prime}$ if either $e=\operatorname{pred}\left(e^{\prime}\right)$ or $e=\operatorname{ante}\left(e^{\prime}\right)$. Such a relation between $e$ and $e^{\prime}$ is denoted $e \rightarrow e^{\prime}$.

4. $e^{\prime}$ is said to depend on the execution of $e$ if there exists a sequence of events $e=e_{0}, e_{1}, \ldots . e_{n}=$ $e^{\prime}$ such that $e_{i} \rightarrow e_{i+1}$ for all $0 \leq i<n$. Such a relation between $e$ and $e^{\prime}$ is denoted $e \Rightarrow e^{\prime}$.

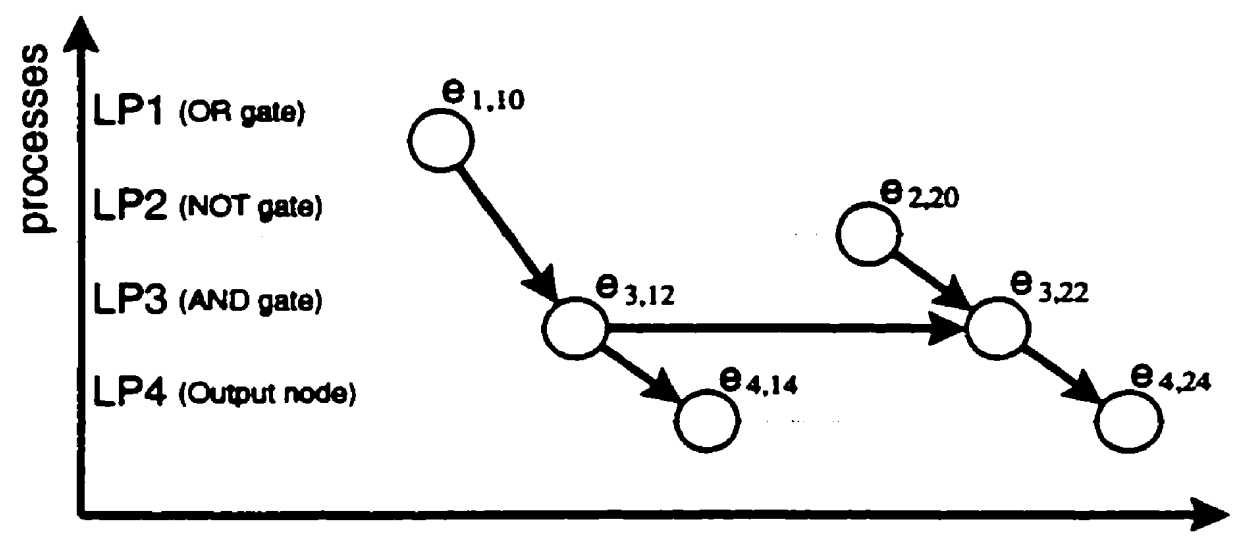

simulation time

Figure 2.6: Space-time diagram of the event dependence graph

The space-time diagram of figure 2.6 represents the dependence relation between the events generated by the LPs in the example given in section 2.2. For instance. event $e_{1.10}$ processed at $L P_{1}$ affects event $e_{3.12}$ to be processed at $L P_{3}\left(e_{1,10} \rightarrow e_{3,12}\right)$. Similarly, the transitive closure of $e_{1.10} \rightarrow e_{3,12}$ and $e_{3,12} \rightarrow e_{4,14}$ induces $e_{1,10} \Rightarrow e_{4,14}$.

The critical path analysis of the event dependence graph of our example highlights the following critical path: $e_{1.10} \rightarrow e_{3.12} \rightarrow e_{3.22} \rightarrow e_{4.24}$. If we assume that it takes $T$ units of real time for a logical process to execute an event. then the overall execution of the simulation will take at least $4 T$ units of real time regardless of the total number of processors used. Since the sequential execution of our simulation on a single processor takes $6 T$ units of real time (ie: 6 events are processed), the upper bound of the speedup that can be obtained by the execution of the simulation on multiple processors is $6 / 4=1.5$. regardless of the number of processors used.

Despite the simplicity of our example, this clearly shows that the success of Parallel Discrete Event Simulation algorithms depends mostly of the parallelism available in VLSI chips. 


\subsubsection{Experiments}

To illustrate the problem of using inadequate metrics to measure the parallelism of a circuit, we performed analysis on four of the largest benchmark ISCAS'89 sequential circuits [Brgl89]. Table 2.2 gives a description of each of these circuits. The ISCAS' 89 benchmark suite is widely used as a basis for studies on digital circuits and major publications adopted it (such as the Design Automation Conference and the Parallel and Distributed Simulation Workshop). The benchmarks were gathered from different industrial and university sources around the world.

\begin{tabular}{|l||c|c|c|c|}
\hline Circuit & \# inputs & \# outputs & \# flip-flops & total \\
\hline \hline s15850 & 14 & $\mathbf{8 7}$ & 597 & 10,470 \\
\hline s35932 & 35 & 320 & 1,728 & 18.148 \\
\hline s3841i & 28 & 106 & 1,636 & 23.949 \\
\hline s38584 & 12 & 278 & 1.452 & 20.995 \\
\hline
\end{tabular}

Table 2.2: ISCAS'89 Benchmark circuits characteristics

We define two types of parallelism:

- The synchronous parallelism which defines the maximum potential speedup achievable by synchronous algorithms such as time-stepped simulators.

- The asynchronous parallelism which gives an upper bound of the speedup achievable by asynchronous algorithms.

\section{Synchronous Parallelism}

We stimulated each of these circuits with a set of random input vectors and all of the events generated during the simulation were logged. Figures 2.7 and 2.8 show the number of concurrent events ( $y$ axis) versus the number of simulation steps ( $x$ axis) for each of the circuits when a new input vector is applied. An input vector is a set of logic values applied to the primary inputs of the circuit. Once the changes have propagated through the combinatorial logic, the clock signal 

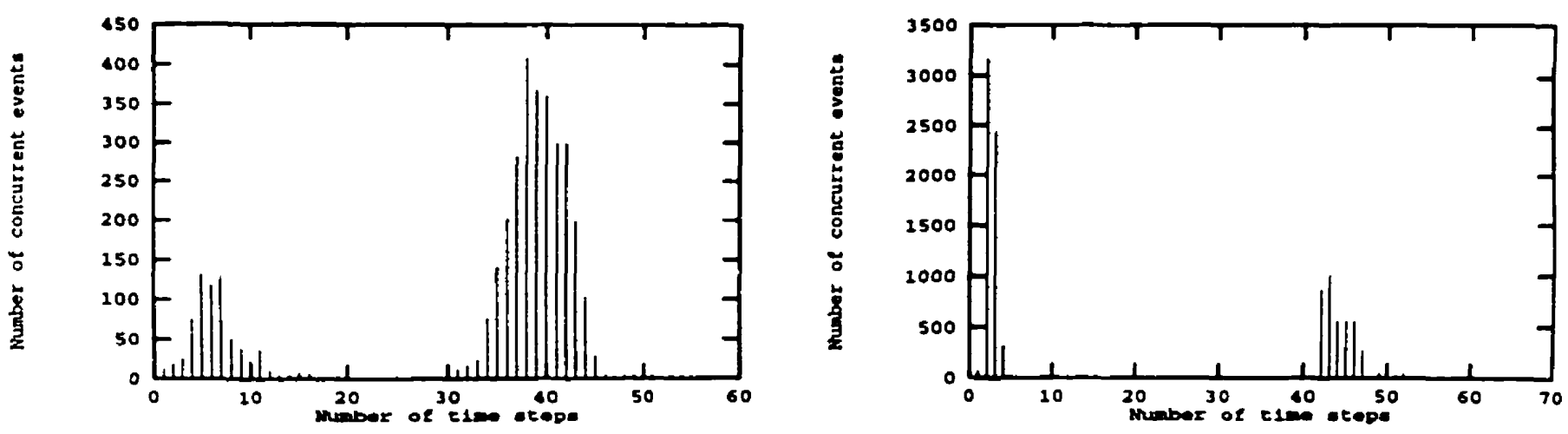

Figure 2.7: Event Concurrency of circuits s15850 (a) and s35932 (b)
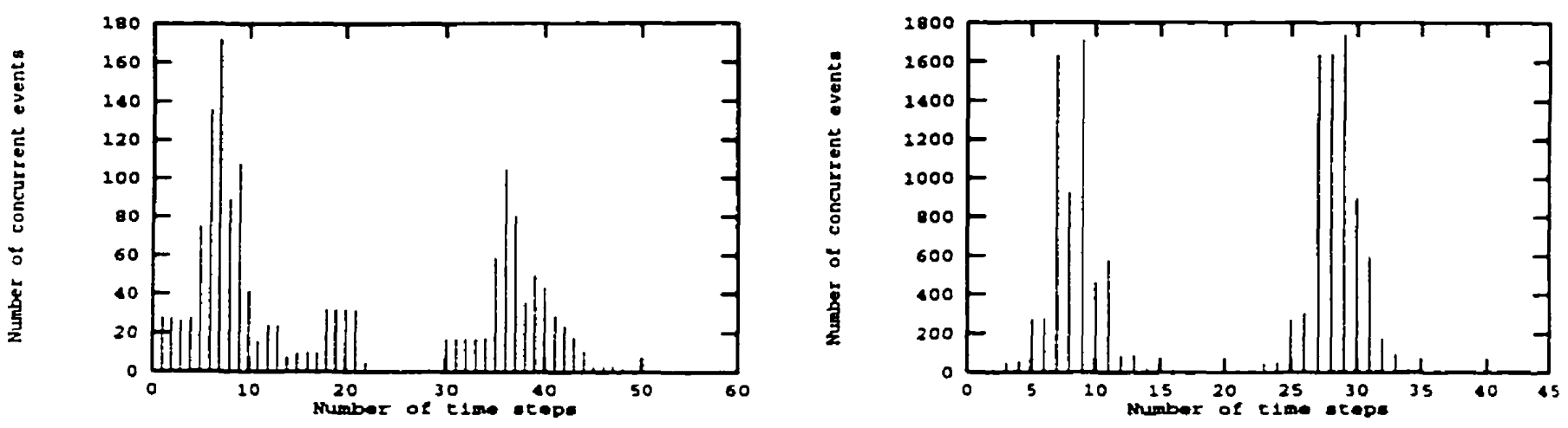

Figure 2.8: Event Concurrency of circuits s38417 (a) and s38584 (b)

rises and the latches are reevaluated. This will cause other changes to be propagated throughout the circuit. For example. in figure 2.ja. the clock signal rises at time step 0. triggering the flipflops. Changes propagate rapidly in the circuit to reach a peak between time steps 5 to $\bar{\tau}$. From time step 8. the event concurrency falls quickly, and remains small for about about 20 steps. The input vector is changed at time step 30 . causing a greater change in the circuit state. The concurrency rises up to 400 events per step, but again, it falls rapidly and dies slowly.

These figures suggest that actually many time steps contain very few concurrent events, implying that computing resources are indeed wasted when synchronous or time-stepped simulation techniques are used.

For each circuit. we calculated the average synchronous parallelism defined as being the total number of events executed during the simulation run divided by the number of clock cycles during 
which at least one event occurred. We also calculated the relative synchronous parallelism defined by Bailey [Bail92b] as being the average synchronous parallelism divided by the total number of elements in the circuit. Table 2.3 shows the synchronous parallelism available in the four largest ISCAS'89 benchmark circuits.

\begin{tabular}{|c||c|c|c|}
\hline \multirow{2}{*}{ Circuit } & \multirow{2}{*}{ Size } & \multicolumn{2}{|c|}{ Synchronous Parallelism } \\
\cline { 3 - 4 } & & Average & Relative \\
\hline \hline s15850 & 10,470 & 76 & $0.72 \%$ \\
s35932 & 18,148 & 474 & $2.61 \%$ \\
s38417 & 23.949 & 43 & $0.17 \%$ \\
s38584 & 20.995 & 328 & $1.56 \%$ \\
\hline
\end{tabular}

Table 2.3: Synchronous Parallelism

Our data correlate Bailey's results. Event concurrency does not necessarily increases as the circuit size increases. For example. s15850 has an activity 4 times higher than that of s3841 7 . even though its size is half that of circuit s3841 $\mathrm{i}$. Considering the size of the circuits we simulated. we also observe that the event concurrency is fairly low for any of them.

\section{Asynchronous Parallelism}

When using an asynchronous simulation algorithm, events occurring at the same simulated time need not to be simulated necessarily at the same real time. This has the advantage of extracting more parallelism from the simulation. To illustrate this problem. each trace generated by the sequential simulation of the benchmark circuits was analyzed and the their event dependence graph $G(V, E)$ was produced. in which each vertex $v_{i} \in V$ represents a processed event and each edge $e_{i, j} \in E$ represents an event dependency (see figure 2.6).

We define the average asynchronous parallelism of a simulation and its associated event dependency graph $G(V, E)$ to be $\|V\| / l_{c p}$ where $l_{c p}$ is the length of the critical path of graph $G(V, E)$. Assuming no communication delay and uniform computation delay, the average asynchronous 
parallelism gives an upper bound of the average number of concurrent events an asynchronous simulator can process at any time.

Similarly to the relative synchronous parallelism, the relative asynchronous parallelism is defined as being the average asynchronous parallelism divided by the total number of elements in the circuit.

Table 2.4 shows the average and the relative asynchronous parallelism of the studied circuits. Results for the entire series of the ISCAS'89 benchmark circuits are given in appendix A.

\begin{tabular}{|c||c|c|c|}
\hline Circuit & Size & \multicolumn{2}{|c|}{ Asynchronous Parallelism } \\
\cline { 3 - 4 } & & Average & Relative \\
\hline \hline s15850 & 10.470 & 658 & $6.28 \%$ \\
s35932 & 18,148 & 1,839 & $10.13 \%$ \\
s38417 & 23.949 & 650 & $2.71 \%$ \\
s38584 & 20.995 & 1.107 & $5.27 \%$ \\
\hline
\end{tabular}

Table 2.4: Asynchronous Parallelism

\subsubsection{Observations}

Figures 2.9 and 2.10 show the average and the relative parallelism respectively for both the synchronous and the asynchronous approach. In most cases. the average number of concurrent events is relatively high, however. depending on the circuit. the average asynchronous parallelism is from 3 to 15 times higher than the average synchronous parallelism. These results clearly show that focusing on the concurrency available at each time step does not necessarily yield a good measure of the inherent parallelism available in digital circuits.

Asynchronous approaches have a major role to play to increase the simulation performance and their success over synchronous techniques depends on the fact that the gain in increased parallelism is not lost by the overhead incurred by the algorithm. 


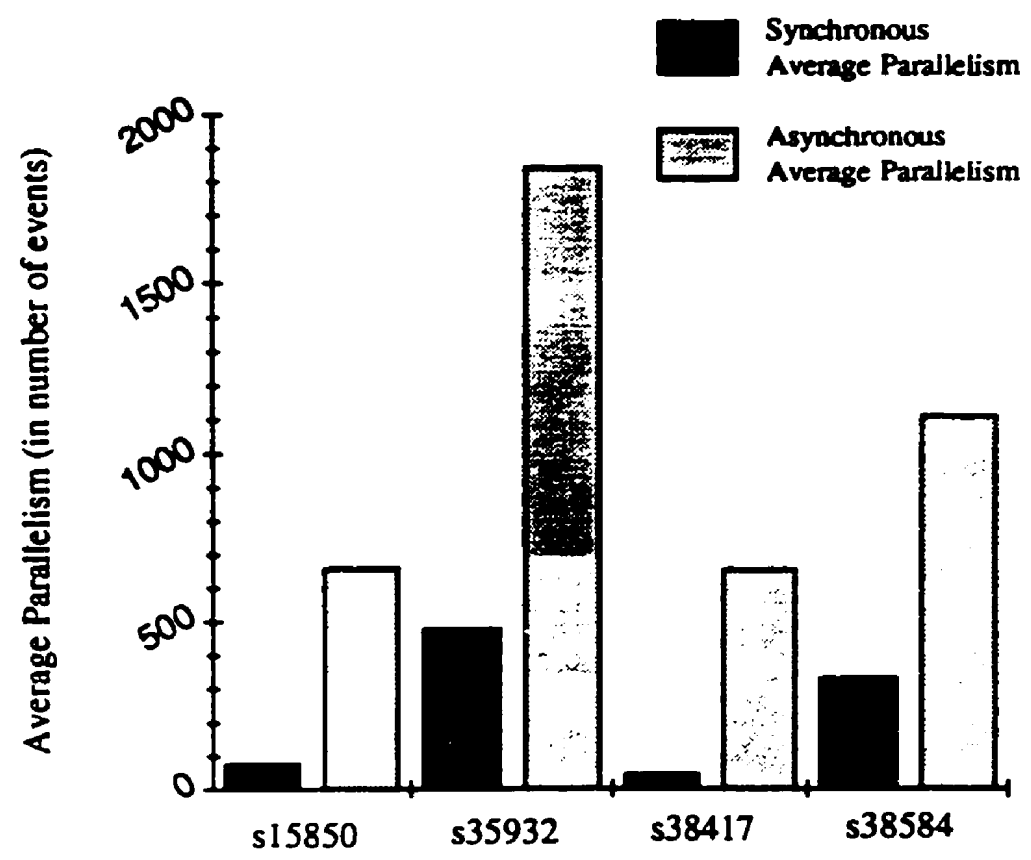

Figure 2.9: Synchronous and Asynchronous Average Parallelism

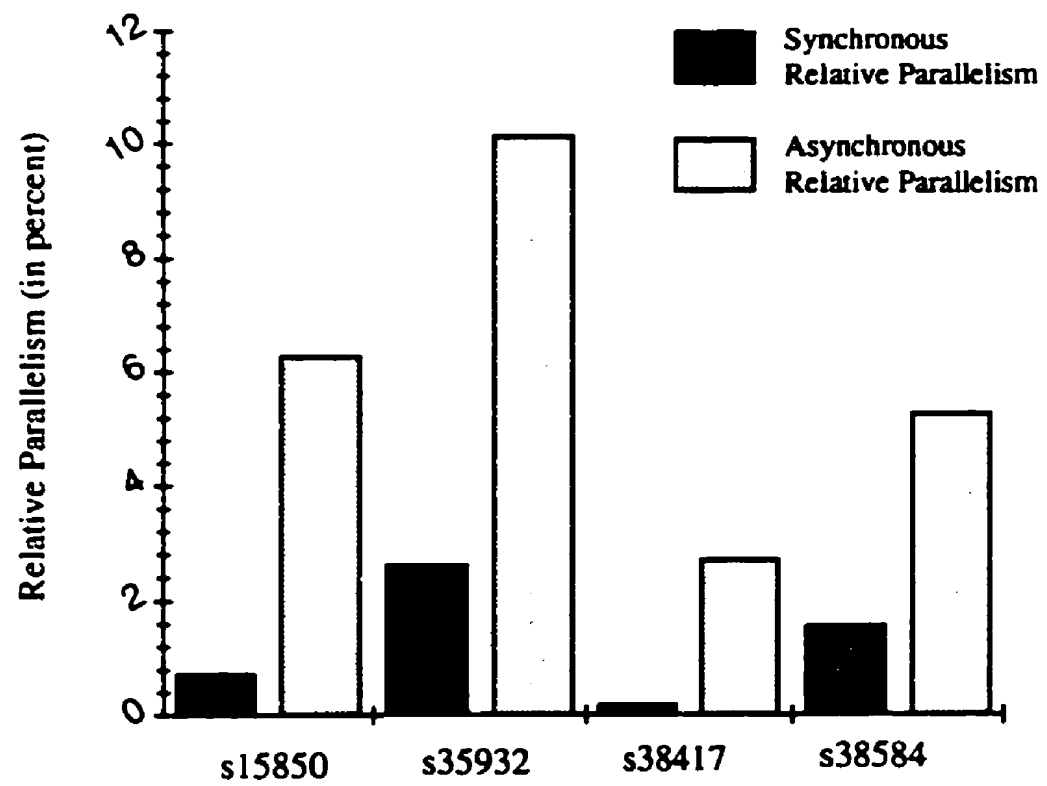

Figure 2.10: Synchronous and Asynchronous Relative Parallelism 


\subsection{Workload Distribution}

Another important factor that can affect the parallel simulation of digital circuits is the distribution of the workload among the processes involved in the simulation. We define the workload of a process as follows:

$$
\lambda_{L P_{1}}=\frac{\Lambda_{L P_{i}}}{\operatorname{Max}\left(\Lambda_{L P_{j}}\right) \forall L P_{j} \in S_{L P}}
$$

where $\Lambda_{L P_{1}}$ is the number of model evaluations performed by process $L P_{i}$ during the simulation run and $S_{L P}$ is the set of all the processes involved in the simulation. The workload $\lambda_{L P}$ is a ratio $\left(0 \leq \lambda_{L P_{t}} \leq 1\right)$ which indicates a heavy load if its value is close to 1 or a light load if its value is close to 0 .
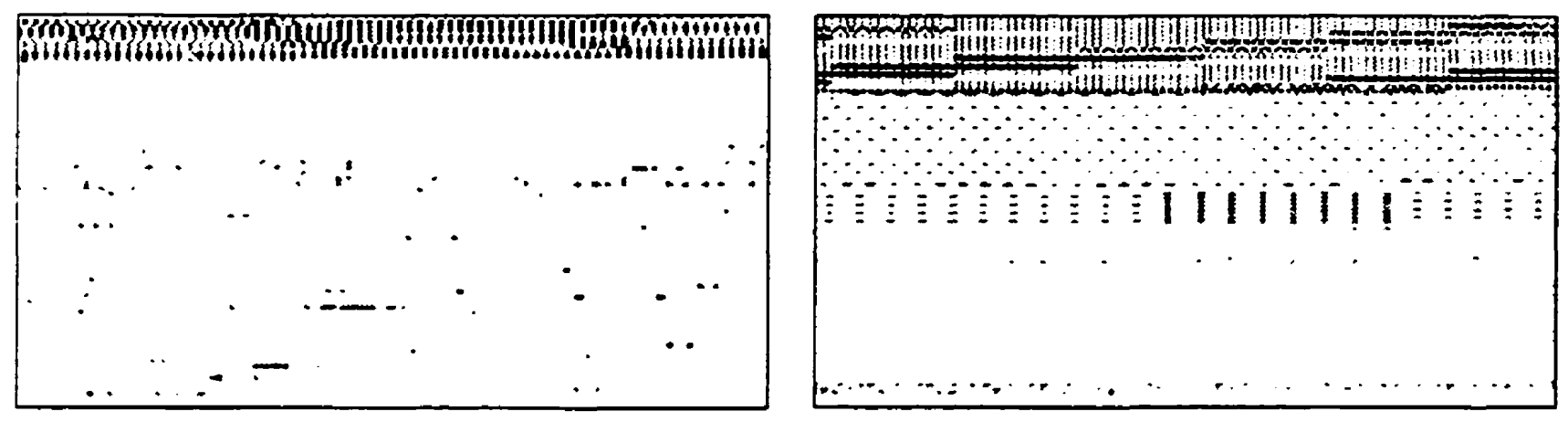

Figure 2.11: Workload distribution for circuits s15850 (a) and s35932 (b)
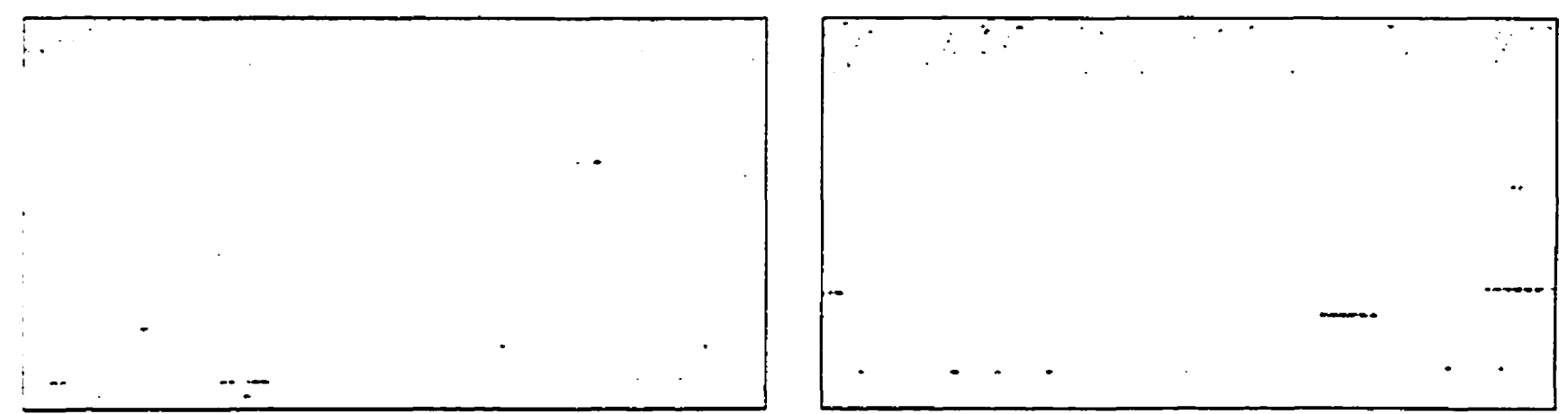

Figure 2.12: Workload distribution for circuits s3841T (a) and s38584 (b) 
The workload of a process depends on the level of activity of the logical gate modelled by that process. If the signal on the input ports changes frequently, the activity of the gate is high, and the workload of the process modelling the gate will also be high. Reciprocally, if the input signals of a gate rarely change, the workload of the corresponding process will be low.

Knowing more about the workload distribution in a simulation is important for partitioning the circuit and balancing the load of the simulation. Because such information is not readily available from the model descriptions, several simulations were ran during which each process kept track of the number of model evaluations it performed. The results are depicted in figures 2.11 and 2.12 .

In each graph. all the processes are layed out, and for each of them, the workload is represented by a pixel. The arrangement of the gates is given by the order in which the gates are specified in the netlist description of the circuit. Clearly, if the gate $G_{i}$ is the $i$-th gate to be defined in the netlist, then its workload will be displayed by the pixel in column $c$ and row $r$ of the graph such that $i=c+\left(r . N_{r}\right)$ where $N_{r}$ is the number of pixels in each row. Note that the gate $G_{0}$ is displayed at the top left corner of the graph.

Each pixel can either be white. light gray, dark gray or black depending on the workload of the process. A white pixel is a process whose workload is less than 0.25 . A light gray pixel is for a workload comprised between 0.25 and 0.5 . If the process' workload is between 0.5 and 0.75 . the pixel is dark gray, and if the workload is above 0.75 , the pixel is turned black. The higher the workload. the darker the pixel.

What stands out from examining these graphs is that the majority of the circuit is idle most of the time. and a very small part gets most of the circuit workload. Furthermore, these figures clearly show that the load is not distributed evenly among the gates of the circuits. For example. circuits s38417 and s38584 (figure 2.12) both exhibit a bad load distribution (ie: their workload is very localized).

Figure 2.13 gives a summary of the results. For each circuit, we show the number of elements very lightly loaded (with a workload $\lambda$ smaller than 0.25 ), as well as those lightly loaded $(0.25<$ $\lambda<0.5)$, heavily loaded $(0.5<\lambda<0.25)$ and those very heavily loaded $(\lambda>0.75)$. For all the circuits we have considered, most of the gates have a very light workload and a very few of them 


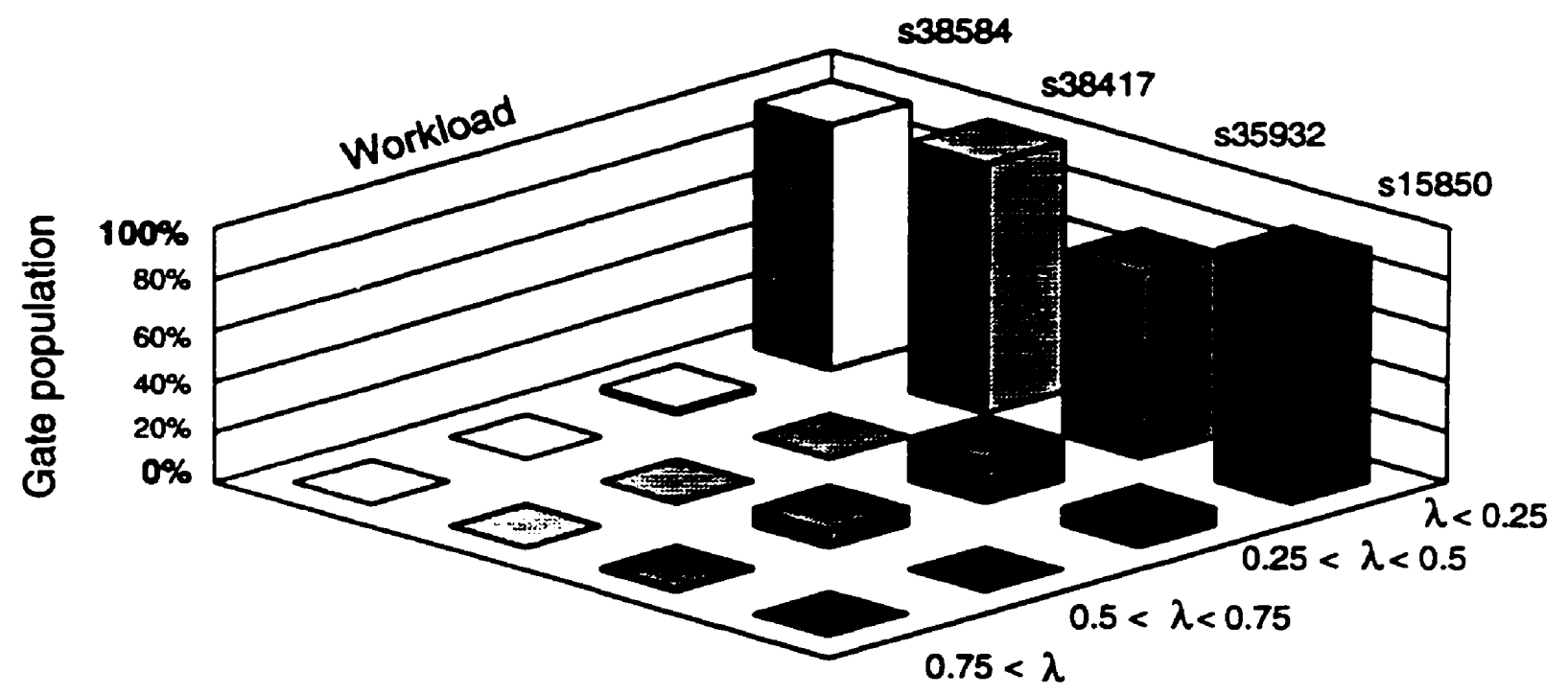

Figure 2.13: Workload distribution

have a very high one. Hence. the reason why most of the partitioning algorithms developed so far do not give entirely satisfactory results for the logic evaluation of VLSI circuits might be that the heuristics used are based solely on the connection graph of the circuit which does not give any information on the workload distribution. This also suggests that a simple partitioning algorithm along with an efficient dynamic load-balancing technique might be a good approach to improving the performance of a logic simulation.

\subsection{Workload Variability}

Little research has been done to determine whether the workload of the internal components of VLSI circuits changes over time. A small variability in the workload for the components of a circuit indicates that the workload distribution over the circuit does not change a lot. Chamberlain and Henderson [Cham94] empirically investigate the use of pre-simulation and try to determine whether the workload (referred to as the evaluation frequency in the paper) during the first $10 \%$ of the simulation is a good prediction of the workload for the rest of the simulation. Their results are actually very positive and show that pre-simulation is an excellent method to 
predict the forecasting of workload. Since their study was done on small size circuits (from 500 to 3,000 elements), the results need to be confirmed for larger circuits.

Taking the same approach as Chamberlain and Henderson, we ran several simulations on the 4 largest circuits of the ISCAS' 89 benchmarks. The simulations were run on 10 processors of the BBN Butterfly using Clustered Time Warp. For each of these circuits, we ran a 300 second simulation on the average. After 30 seconds, the workload for each gate was taken and then compared to the total workload at the end of the simulation. The difference between the two values was calculated for each gate.

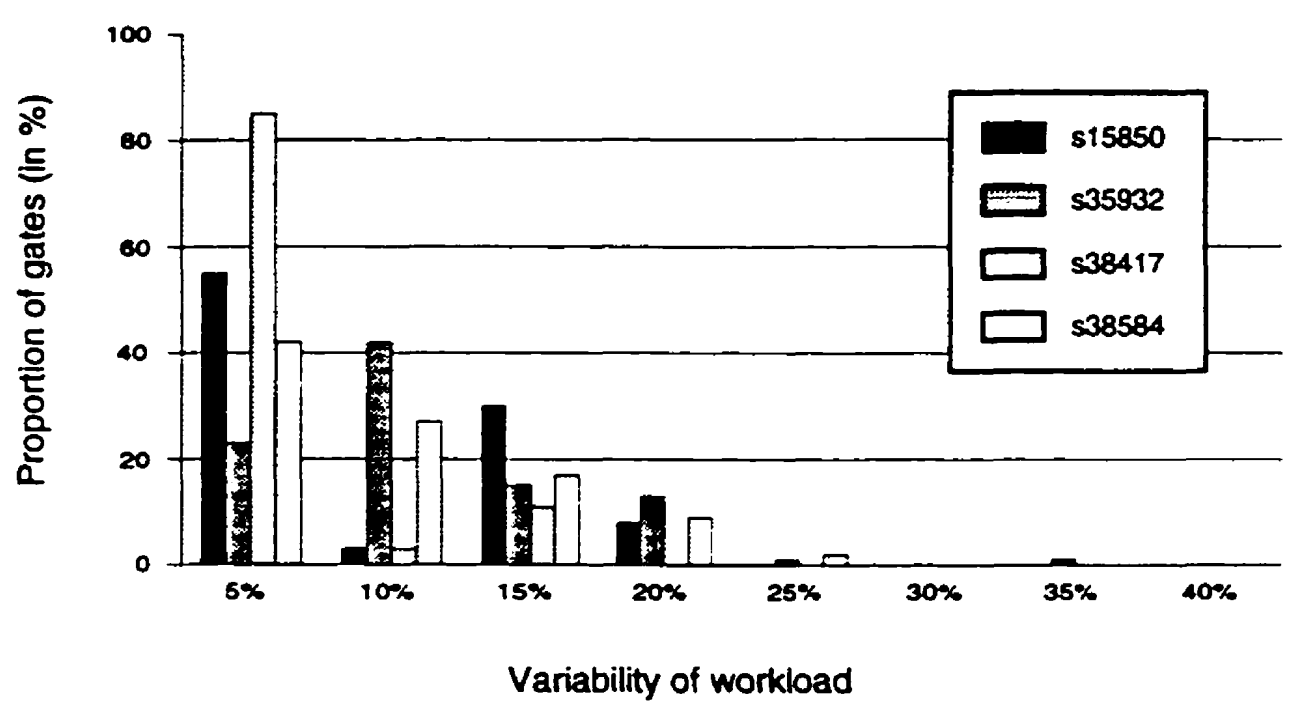

Figure 2.14: Workload Variability

Figure 2.14 shows the distribution of these differences for all of the circuit components. We can see that for each circuit. the workload measured at the end of the pre-simulation (10\% of the whole simulation) does not vary much during the rest of the simulation. For most of the gates. the variation of their workload stavs within a range of $10 \%$ and less. These results imply that pre-simulation is a good way to predict the workload distribution of a circuit and can therefore be effectively used to devise partitioning and load-balancing algorithms. 


\subsection{Feedback Loops}

Digital circuits have feedback loops, thereby creating cycles in the dependency graph. The importance of these cycles can have a pronounced effect on the efficiency of the simulation system whether a conservative or an optimistic algorithm is used. In the first case, a large number of cycles increases the risk for deadlocks, hence augmenting the overhead incurred by the detection and the breaking of these deadlocks. In the case of the optimistic approach. cycles increase the risk of rollbacks and consequently the risk of rollback explosion.

\section{Inputs}

$V$ is a finite set of vertices.

$E$ is a binary relation on $V$ whose elements are edges. $G(V, E)$ is a digraph.

\section{Temporary Variables}

color $r_{i}$ is the color associated with vertex $v_{i}$. Is is either WHITE ( $v_{i}$ not visited),

GRAY ( $v_{i}$ being visited) or BLACK ( $v_{i}$ visited).

$v_{i, l}$ is the number of all the cycles of length $l$ starting at vertex $v_{i}$

less the number of cycles of length $l$ including vertex $v_{j} \forall j<i$.

$\Psi_{i}$ is the set of all $\psi_{i, l}$ elements for vertex $i$ and for all lengths $l$.

Output

$\phi_{l}$ is the number of cycles of length $l$ in the graph.

\section{begin}

(1) $i \leftarrow 0$

(2) while $i<|V|$ do

(3) for all $v_{j} \in V$ such that $j \geq i$ do

(4) color $_{j} \leftarrow$ WHITE

(5) $\quad \Psi_{j} \leftarrow \emptyset$

$$
\text { endfor }
$$

(6) ModifiedBFS $\left(v_{i}, v_{i}\right)$

(7) for each $\psi_{i, l} \in \Psi_{i}$ do

(8) $\quad \phi_{l+1} \leftarrow \phi_{l+1}+\psi_{i, l}$

$$
\text { endfor }
$$

(9) $i \leftarrow i+1$

$$
\text { endwhile }
$$

end.

Figure 2.15: Pseudocode of the main procedure of the cycle inventory algorithm 


\section{Inputs}

root is the vertex from which the search originally started.

$v_{i}$ is the vertex from which a new search should be started.

\section{Outputs}

Returns True if paths to root are discovered, otherwise returns False

ModifiedBFS $\left(\right.$ root,$\left.v_{i}\right) \rightarrow$ Boolean

begin

(1) if color $_{i}=$ BLACK then return True

(2) if color $_{i}=$ GREY then return False

(3) color $_{i} \leftarrow$ GREY

(4) for each $v_{j} \in G(V, E)$ such that $v_{j}$ is a predecessor of $v_{i}$ do

(5) if $v_{j}=$ root then

(6) $\quad w_{i, 1} \leftarrow v_{i, 1}+1$

(7) if $j>i$ then

(8) if ModifiedBFS $\left(\right.$ root. $\left.v_{j}\right)=$ True then

(9) for each $w_{j, l} \in \Psi_{j}$ do

$$
v_{i, l+1} \leftarrow v_{i, l+1}+v_{j, l}
$$

$$
\text { endfor }
$$

endif

endif

endfor

(11) color $_{i} \leftarrow$ BLACh

(12) return True

end.

Figure 2.16: Pseudocode of the ModifiedBFS procedure of the cycle inventory algorithm

For this reason. the number of cycles as well as their length were calculated for the ISCAS'89 benchmark circuits. We made use of a "cycle inventory" algorithm whose pseudocode is given in figures 2.15 and 2.16 .

This algorithm. based on the breadth-first search approach [Corm91], calculates the number of occurrences of simple cycles of any given length. In a directed graph, a simple cycle is a path $\left\langle v_{0}, v_{1}, \ldots v_{k}\right\rangle$ which contains at least one edge and where $v_{0}=v_{k}$, and $v_{1}, \ldots, v_{k-1}$ are all distinct. Each vertex $v_{i}$ has a color, color $r_{i}$ which can either be white, grey or black. A vertex is colored white, if it has not been visited yet by the algorithm. If it is colored gray, the vertex is 
currently being visited by the algorithm. Otherwise, if the vertex has already been visited, its color is black. Each vertex $v_{i}$ has a cycle set $\Psi_{i}$ which contains a finite number of elements $\psi_{i, l}$ such that $\psi_{i, l}$ is the number of simple cycles of length $l$ starting at vertex $v_{i}$.

The main procedure visits each node $v_{i}$ in the vertex set $V$ starting at node $v_{0}$ (line 1 and 2). In lines 3,4 and 5, all the nodes whose index is larger than or equal to $i$ are whitened and their cycle set is emptied. Then the graph is visited starting at vertex $v_{i}$ using a breadth-first search algorithm. In lines 7 and 8 , the results of the partial search are merged with the global results, and the algorithm proceeds with the next vertex. Intuitively, on the first pass, all the cycles which include vertex $v_{0}$ are visited and counted. In the second pass, all the cycles which include $v_{1}$ are visited but only those which do not include $v_{0}$ are counted. Similarly, in the third pass, all the cycles including vertex $v_{2}$ are visited but only those which do not include $v_{0}$ and $v_{1}$ are counted. And so on until all the nodes have been visited by the main procedure.

The purpose of the ModifiedBFS procedure is to calculate the number of all possible paths whose first vertex is root and last one is $v_{z}$ (ie: $v_{i}$ is reachable by root). In addition, the length of each of these paths is also calculated. The algorithm checks whether any of the predecessors of $v_{i}$ is root. If that is the case. a new path of length 1 is detected and added to $\Psi_{i}$ (line 6). Otherwise. the procedure recursively calls itself and merges the results if new paths are discovered (line 9 and 10 ). Line $\bar{\tau}$ prevents the algorithm from re-counting cycles which include vertices that have been already visited by the main procedure.
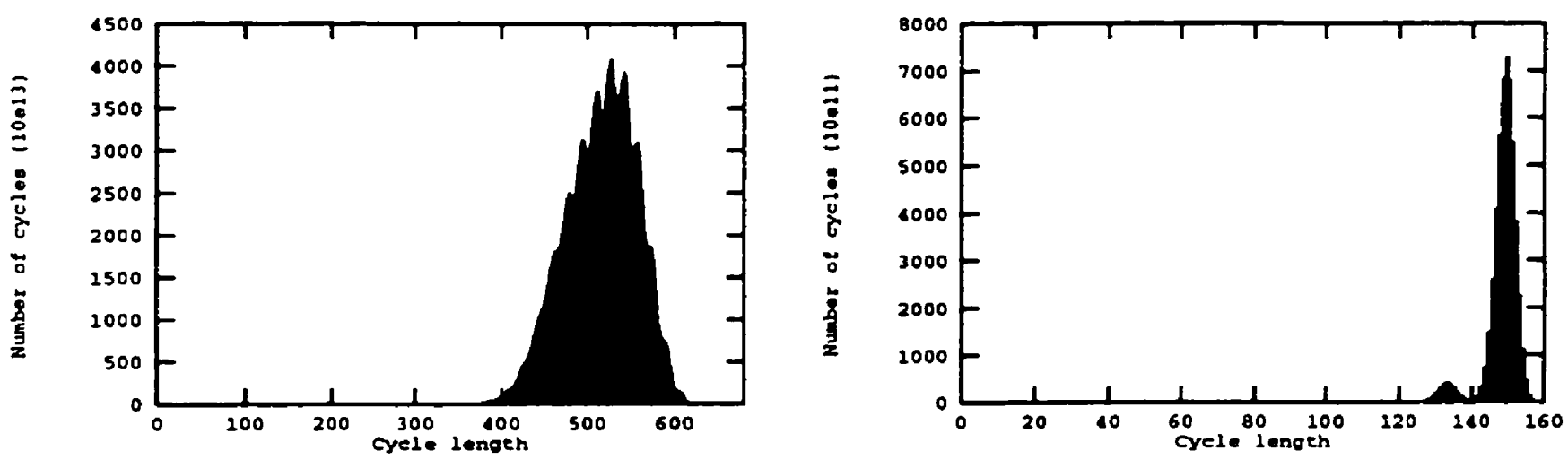

Figure 2.17: Cycle distribution for circuit s15850 and s35932 

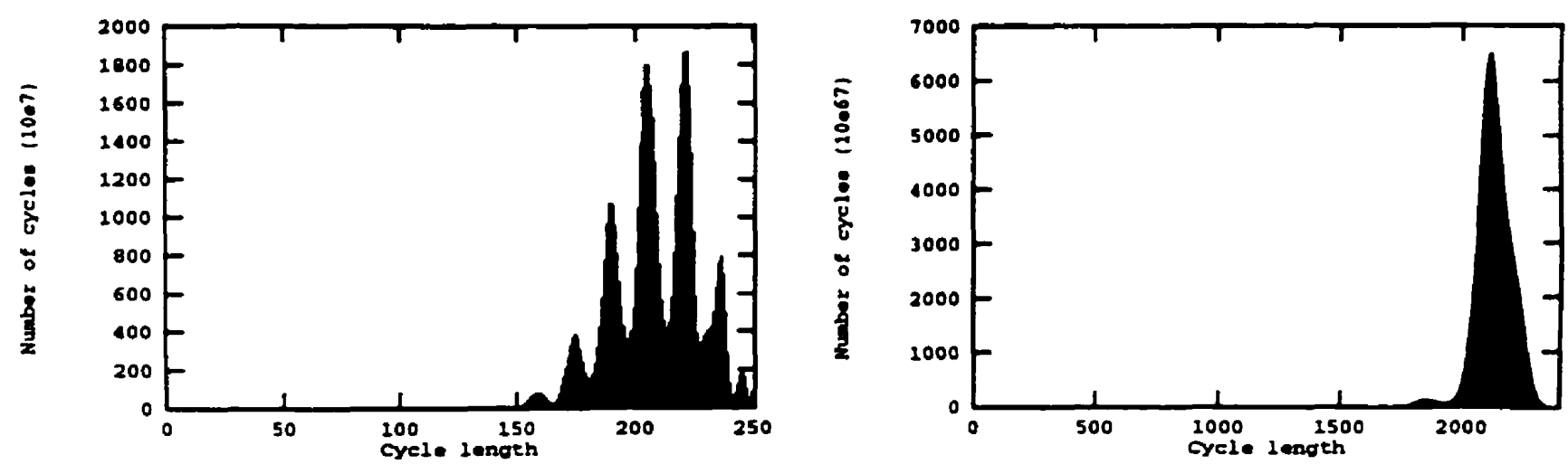

Figure 2.18: Cycle distribution for circuits s38417 and s38584

Results are depicted in figures 2.17 and 2.18. We observe that each circuit contains a very large number of cycles of variable sizes. For example, s38584 has approximately $10^{70} \mathrm{cycles}$ with a length of about 2.000 gates. These data reveal that the large number of cycles can be a major issue for any of the asynchronous algorithms discussed above. Trying to avoid interprocessor cycles becomes a difficult task. In addition to a high time complexity, it is unsure whether an algorithm whose objective is to minimize interprocessor cycles can have a significant impact due to the large number of cycles to be considered.

\subsection{Summary}

In addition to characteristics specific to logic simulation such as fixed routing. low computational granularity, fixed-gate delay, the following points have been clarified:

1. Room for improvement. Even though the average number of concurrent model evaluations per time step is rather small, we have shown that asynchronous algorithms can extract more parallelism than synchronous algorithms. Depending on the circuit considered (see table 2.2), the potential speedup achievable by an asynchronous algorithm is 3 to 15 times higher than that of a synchronous algorithm such as a time-stepped technique.

2. Unbalanced workload distribution. In the circuits we have analyzed, a majority of the gates have a very light workload while a minority are extremely busy. Consequently, 
a partitioning algorithm or a dynamic load-balancing algorithm should take this into consideration in order to yield good performance.

3. Small workload variability. Pre-simulation can be used to extract information such as workload distribution and inter-processor communication. This can be extremely useful for problems such as partitioning, mapping and dynamic load-balancing since that type of information is not known in advance.

4. Large number of cycles. The presence of a very large number of cycles in the circuits is likely to increase the overhead of asynchronous algorithms, either because of deadlocks for a conservative approach or because of rollbacks for an optimistic approach.

5. Circuits are structured. Due to the hierarchical approach designers use to develop their systems, circuits are structured and can be decomposed into functional units. These functional units are generally interconnected by latches driven by clock signals. Therefore, partitioning can benefit from the implicit hierarchical structure of the design. 


\section{Chapter 3}

\section{Clustered Time Warp}

\subsection{Introduction}

In this chapter. we introduce a new distributed asynchronous algorithm for the simulation of large digital circuits on general purpose distributed-memory multiprocessor architectures.

As we have seen previously: there are three major approaches to parallel simulation: the conservative. the optimistic and the space-time approach.

Conservative algorithms are known to have a low memory usage. On the other hand. avoiding or detecting and breaking deadlocks can reduce greatly the performance, especially when large models with small computational granularity such as those found in the domain of logic simulation are considered. In general. conservative algorithms are overly pessimistic and depend a great deal on lookahead to achieve good performance [Fuji90b]. Given the large number of cycles in a circuit (see chapter 2). this might present a serious drawback. Another disadvantage of most conservative approaches is that the interconnection graph of the processes must be statically defined.

Optimistic algorithms are very attractive for logic simulation since they can extract a great deal of parallelism and they are deadlock-free. Nevertheless. Time Warp studies often pointed out the problems encountered due to the large amount of memory a simulation might require. Furthermore, it is unclear whether Time Warp remains efficient as the size of the simulation model grows.

It is difficult to map the space-time approach to the logic simulation problem. Space-time is more appropriate for problems in which objects are moving in a given space and in which there 


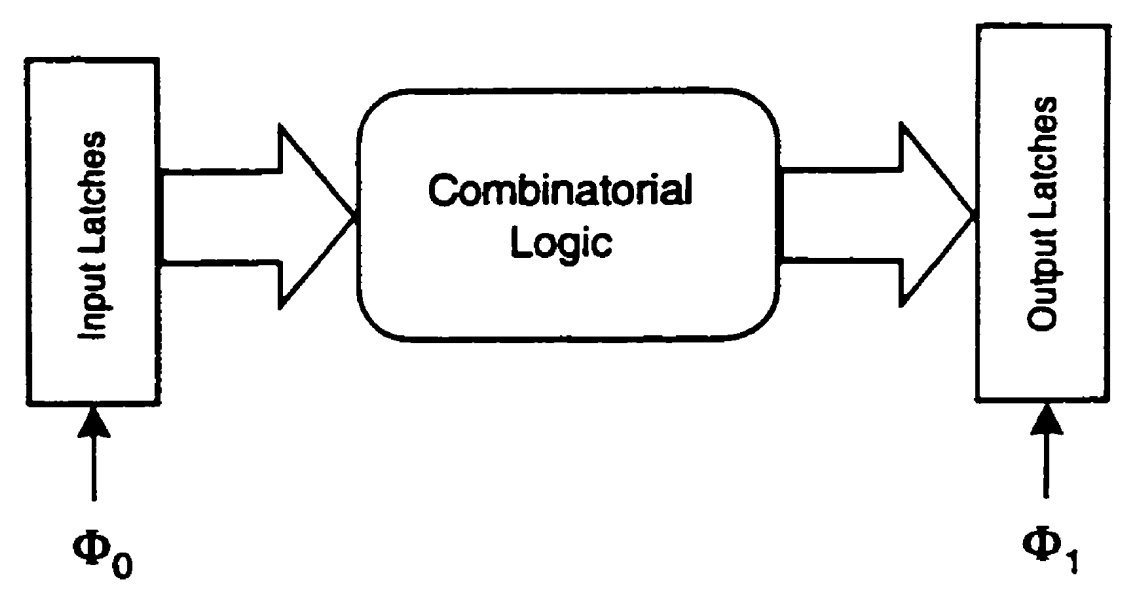

Figure 3.1: General Circuit Structure

is a certain regularity in the problem.

The ideal algorithm would be one that would have the memory needs of conservative algorithms and the potential of optimistic algorithms to extract a great deal of parallelism.

In chapter 2 , we have seen that digital circuits are constructed by interconnecting functional units. which are themselves composed of different blocks. At the lowest level a block can be modeled as some combinatorial logic connected to a series of clocked registers or latches.

Figure 3.1 illustrates the hardware model of logic circuits [Schu89]. We distinguish three phases:

1. An initialization vector is applied to the input latches and once the signal is stable, clock $\Phi_{0}$ is activated.

2. The propagation vector travels throughout the combinatorial logic and reach the output latches.

3. The output vector is then sampled when the clock $\Phi_{1}$ is activated.

This suggests that the signal activity within the blocks is rather chaotic whereas the activity between the blocks tends to be more regular. The key idea would then be to use a conservative approach to synchronize all the gates of one block, and to use an optimistic approach to synchronize these blocks. 
In this chapter, we are going to present a new hybrid algorithm for the asynchronous simulation of digital circuits (the algorithm can of course be applied to other types of simulations). The algorithm makes use of Time Warp between clusters of LPs running on different processors and use a sequential algorithm within the clusters.

\subsection{The Clustered Time Warp Algorithm}

\subsubsection{Clusters}

In the Clustered Time Warp approach, the model is partitioned into clusters of LPs prior to the simulation. The motivation behind this idea is that logical processes modelling the gates which belong to the same functional unit can be grouped together. There is no restriction put on the size and on the number of clusters except that one cluster must reside on a single processor and cannot be split among processors. Each cluster is associated with a Cluster Environment (CE) which is in charge of scheduling the LPs. The Cluster Environment also takes care of all the communication with the other clusters and as a consequence, the $\mathrm{CE}$ manages an input queue and an output queue called the Cluster Input Queue (CIQ) and the Cluster Output Queue (COQ) respectively.

\subsubsection{Events}

When an LP sends an event to another LP located in a different cluster. it gives that event to the Cluster Environment. which keeps a copy of it in its Cluster Output Queue as an antimessage just like an LP would do in a pure Time Warp environment. The CE then sends the event to the appropriate cluster which hosts the destination LP of the event. When the receiving cluster gets the event, its CE simply enqueues it in the CIQ. Such events which cross the cluster boundaries are referred to as external events. If an LP sends an event to another LP which is located in the same cluster. then it passes by the Cluster Environment and enqueues the event directly into the input queue of the receiving LP. Events whose sending and receiving processes are located in the same cluster are referred to as internal events.

Events in the CIQ are sorted by increasing order of receive time whereas events in the COQ are sorted by decreasing order of sending time. The reason why different ordering strategies 
are used is simple. In a pure conservative approach, an event contains only one timestamp that represents the moment at which that event occurred in the physical system. Processes sort the received events by increasing order of timestamp so as to be able to easily retrieve the event with the smallest timestamp value. In an optimistic approach, a process has two types of queues. An input queue which stores received events in a similar way to a conservative system, and an output queue which stores copies of events sent to other processes. When a straggler is received, the process rolls back by restoring an earlier state and sends antimessages. During this last operation, the process goes through its output queue to locate copies of events which were caused by messages whose receive time was larger than that of the straggler. In order to make this operation efficient, events stored in the output queues need to be sorted in decreasing order of sending time.

In Clustered Time Warp, a message has nearly the same structure as in Time Warp. It contains the identification of the sending LP and that of the receiving LP. a sign to differentiate messages from antimessages. a send time and a receive time, and the data needed for model evaluation. The difference with Time Warp lies in the way logical processes are identified. In Time Warp. an LP is identified in the whole system by a single name. In Clustered Time Warp. it is composed of two names: one that identifies the cluster and one that identifies the LP in the cluster. This naming methodology: as we will see in chapter 5. makes the implementation of a dynamic loadbalancing algorithm much simpler. Instead of keeping a routing table in each processor of all the logical processes in the system. all that is needed is to keep the location of the cluster. which will then be in charge of forwarding the event to the appropriate LP. If the cluster happens to have been moved to another processor. only one entry needs to be changed in the routing table instead of changing the entries of all the LPs contained in that cluster.

There are three different types of messages in our simulation system: normal messages which contain the events generated by the simulation itself, antimessages which are necessary to cancel wrong computations. and cont-ol messages which are needed to perform distributed computation such as the calculation of the GVT estimate, termination detection or collection of statistics.

In a system working under proper conditions, normal messages are the dominant source of communication overhead. Antimessages and control messages are comparatively less frequent 
but their transmission delay is far more critical than that of normal messages. For example, the longer an antimessage takes to reach its destination, the more useless work the system is likely to perform, therefore the longer it will take to cancel that work. Similarly, the longer a GVT token takes to be passed around, the less accurate is the GVT estimate, hence making the fossil collection mechanism less efficient. It is therefore necessary for antimessages and control messages to be given a higher priority than other normal messages in order to ensure their fast delivery, especially when the traffic is heavy. Our simulation system is assumed to rest upon a network layer which provides reliable communication channels between the processors and in which messages can have different priority. Furthermore, the Clustered Time Warp approach does not assume a communication system with FIFO properties.

\subsubsection{Scheduling}

The Cluster Environment is responsible for scheduling the LPs in the cluster and each processor schedules all its CEs. A smallest timestamp first scheduling policy is used since it reduces the number of rollbacks. Lin and Lazowska [Lin91] do a thorough study of the scheduling problem in which they confirm the advantage of the smallest timestamp first policy and they even suggest making it preemptive. As a consequence. all the events stored in the CIQ and in the LP's input queues are also put in a priority heap. The event at the top of the heap is the one which has the smallest timestamp; hence the destination LP of that event will be the next process to be scheduled in the cluster.

\subsubsection{Timezones}

Since Time Warp is used between clusters, stragglers may arrive at any time. Therefore a mechanism must be created in order for the Cluster Environment to determine which LPs to roll back and which antimessages to send to cancel incorrect computations. This task is achieved through the use of timezones.

From the cluster's point of view, the simulation is decomposed into a series of adjacent and nonoverlapping time intervals called timezones. When the simulation starts, each cluster has only one timezone with interval $[0,+\infty[$. Each time a cluster receives a message from another cluster 
whose receive time is $t$, it finds the timezone interval $\left[t_{i}, t_{i+1}\right.$ [ into which $t$ fits (ie: $t_{i}<t<t_{i+1}$ ) and splits it into two new timezones with intervals $\left[t_{i}, t\left[\right.\right.$ and $\left[t, t_{i+1}[\right.$. Timezones are then stored in a table in increasing order of time.

\subsubsection{Logical Processes}

Logical processes have a single input queue and no output queue. They also maintain their own logical clock whose value is called the Local Simulation Time (or LST). The behavior of the clock is similar to that of a process' clock in a pure conservative system. If a process $L P_{i}$ with clock $L S T_{i}$ is about to consume message $m_{p}$ with timestamp $t\left(m_{p}\right)$, then the following operations are performed:

1. $L S T_{i} \leftarrow \max \left(L S T_{i}, t\left(m_{p}\right)\right)$.

2. $L P_{i}$ processes $m_{p}$.

3. $L S T_{i} \leftarrow L S T_{i}+$ service time.

Furthermore. the LP also keeps track of the Timestamp ${ }^{1}$ of the Last Event it processed (or TLE). The TLE is different from the LVT (Local Virtual Time) introduced by Jefferson [Jeff85]. In Time Warp. the LVT corresponds to the timestamp of the next event the logical process is going to consume, whereas in Clustered Time Warp, the TLE value corresponds to the timestamp of the last event the LP processed.

When an LP is scheduled for processing, it first checks into which timezone the receive time of the event it is going to consume fits. If that timezone is different from that of the last event the LP processed. then the LP performs a checkpoint by saving its state. Otherwise it directly consumes the event. In short. the LP checkpoints each time it changes timezones.

Each LP is therefore composed of a process in charge of the actual event evaluation. a Local Simulation Time (LST), the Time of the Last Event it processed (TLE), a message input queue. and a state queue.

Figure 7.8 shows the structure of a cluster.

\footnotetext{
${ }^{1}$ Receive time
} 


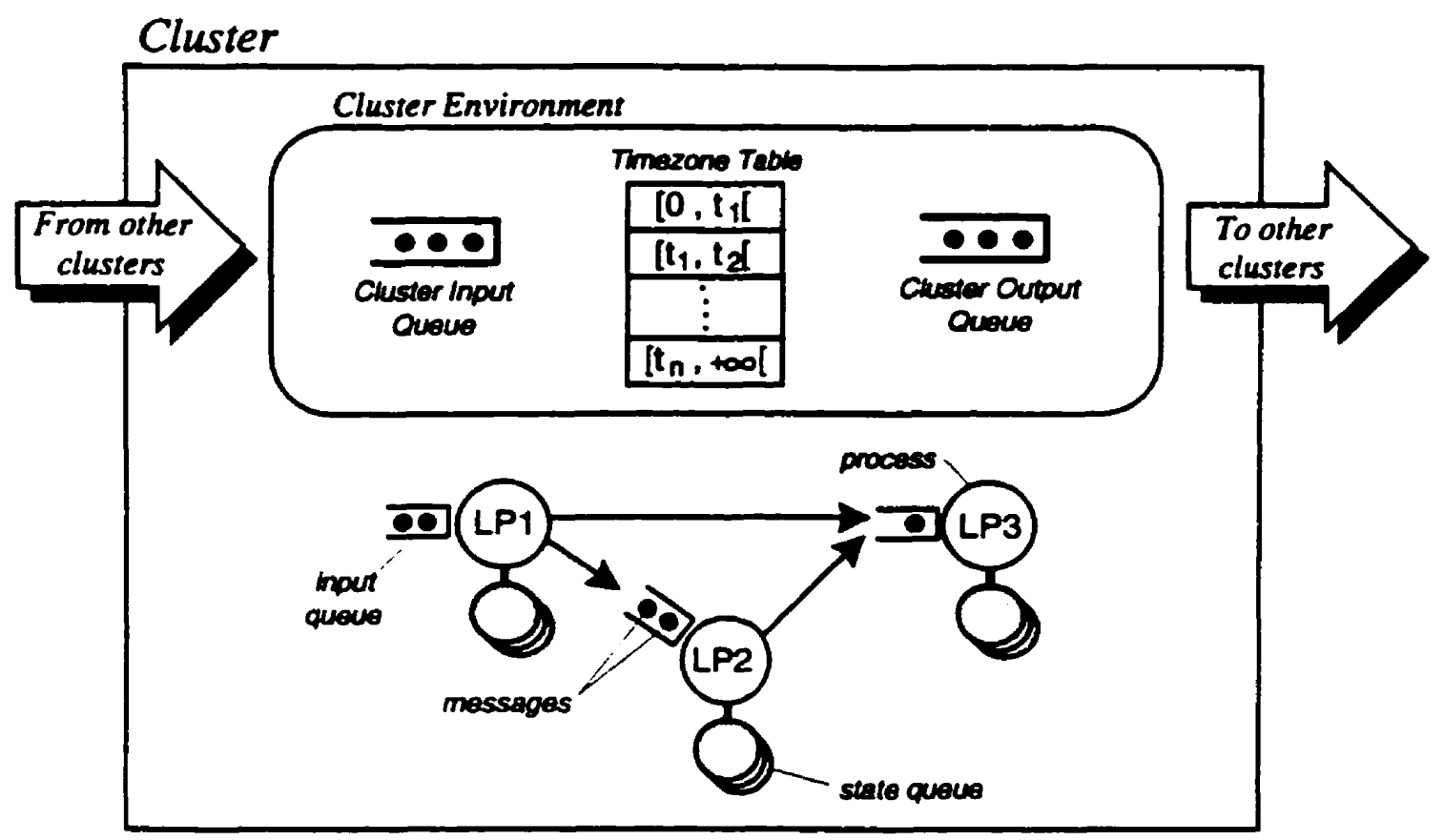

Figure 3.2: Cluster Structure

\subsubsection{Rolling back}

Suppose the cluster receives a straggler with receive time $t_{\theta}$. As we have just seen, the Cluster Environment creates a new timezone for the straggler. It then rolls back all the LPs in the cluster which have a TLE greater than $t_{s}$ to a checkpoint prior to $t_{s}$. In addition, the CE will send all the necessary antimessages stored in the $\mathrm{COQ}$ whose sending time is greater than $t_{s}$. The Cluster Environment proceeds similarly when the cluster receives an antimessage timestamped $t_{a}$ with the difference that instead of creating a new timezone, the CE merges timezones $\left[t_{i}, t_{a}[\right.$ and $\left[t_{a}, t_{i+1}\left[\right.\right.$ into a single one whose interval is $\left[t_{i}, t_{i+1}[\right.$.

Since LPs do not perform a checkpoint every time they process an event. they might have to roll back to a state well before the receive time of the straggler or the antimessage received by the cluster. Therefore LPs need to coast forward as in Time Warp, re-processing all events whose receive time is prior to $t_{s}$, and not resending messages already produced before $t_{s}$. The major difference with Time Warp is that LPs can remove from their input queue all of the internal messages which have a send time greater than the timestamp of the straggler or the antimessage which caused the rollback. This does not affect the correctness of the simulation as 
all the LPs in the cluster are rolled back. Hence, all of the necessary internal messages will be regenerated. Note that the external messages stored in the Cluster Input Queue are not removed since their sending processes are located in different clusters, as a consequence, such messages are not regenerated.

Because the events in the cluster are processed in strict timestamp order (i.e. lowest timestamp first). the descendents of the straggler will be placed correctly in the heap, and events at all of the LPs in the cluster will be processed in the correct order. It is important to note that individual LPs never send antimessages.

\subsection{Pseudocode}

\subsubsection{Pseudocode for the Logical Process}

Figures 3.3 and 3.4 give the pseudocode executed by each logical process in a Clustered Time Warp system. 


\section{Inputs}

$L S T$ is the Local Simulation Time of the logical process.

$T L E$ is the receive time of the last event processed by the logical process.

$e$ is the event to be processed where $t_{r}(e)$ is its receive time and $t_{s}(e)$ is its send time.

\section{begin}

(1) select timezone $Z_{L P}$ with interval $\left[t_{i}, t_{i+1}\left[\mid T L E \in Z_{L P}\right.\right.$

(2) if $t_{r}(e) \notin Z_{L P}$ then checkpoint

(3) $T L E \leftarrow t_{r}(e)$

(4) $\quad L S T \leftarrow \max (L S T . T L E)$

(5) simulate event $e$

(6) $L S T \leftarrow L S T+$ service time

(i) for all events $e^{\prime}$ to send do

(8) $t_{s}\left(e^{\prime}\right) \leftarrow T L E$

(9) $\quad t_{r}\left(e^{\prime}\right) \leftarrow L S T$

(10) if destination LP of $e^{\prime}$ is in the same cluster then

(11) insert $e^{\prime}$ into the input queue of the destination LP

(12) give $e^{\prime}$ to the $\mathrm{CE}$ for it to send endif

endfor

end.

Figure 3.3: The Logical Process is about to process event $e$ 


\section{Inputs}

$\Psi$ is the state queue of the logical process.

$\Omega$ is the input queue of the logical process.

$t_{\text {rbk }}$ is the timestamp to which the process should roll back.

\section{begin}

(1) for each state $S \in \Psi \mid T L E(S)>t_{\text {rbk }}$ do

(2) $\Psi \leftarrow \Psi-\{S\}$

endfor

(3) select state $S \in \Psi \mid T L E(S)=\operatorname{Min}\left(T L E\left(S^{\prime}\right)\right) \forall S^{\prime} \in \Psi$

(4) restore state $S$

(5) for each event $e \in \Omega \mid t_{s}(e)>t_{\mathrm{rbk}}$

(6) $\Omega \leftarrow \Omega-\{e\}$

endfor

\section{${ }^{*}$ Coast Forward */}

(i) while $t_{r}(e)<t_{r b k}$ where $e$ is the next event to process

(8) $T L E \leftarrow t_{r}(e)$

(9) $\quad L S T \leftarrow \max (L S T, T L E)$

(10) simulate event $e^{\prime}$

(11) $\quad L S T \leftarrow L S T+$ service time endwhile

end.

Figure 3.4: The LP is told by the CE to rollback to time $t_{\text {rbk }}$ 


\subsubsection{Pseudocode for the Cluster Environment}

Figures 3.5 and 3.6 give the pseudocode executed by each cluster environment in a Clustered Time Warp system.

\section{Inputs}

$e$ is the event to be processed where $t_{r}(e)$ is its receive time and $t_{s}(e)$ is its send time. $C O Q$ is the Cluster Output Queue.

\section{begin}

(1) delete timezone $Z_{i}$ with interval $\left[t_{i}, t_{i+1}\left[\mid t_{r}(e) \in Z\right.\right.$

(2) if $e$ is an antimessage then

(3) delete timezone $Z_{i-1}$ with interval $\left[t_{i-1}, t_{i}[\right.$

(4) create timezone $Z_{i-1}^{\prime}$ with interval $\left[t_{i-1}, t_{i+1}[\right.$ else

(5) create timezone $Z_{i}^{\prime}$ with interval $\left[t_{i}, t_{r}(e)[\right.$

(6) create timezone $Z_{i}^{\prime \prime}$ with interval $\left[t_{r}(e), t_{i+1}[\right.$ endif

(i) for each LP in the cluster with a $T L E \geq t_{r}(e)$ do

(8) tell LP to roll back to a state prior to $t_{r}(e)$ endfor

(9) for each antimessage $\bar{e} \in C O Q \mid t_{s}(\bar{e}) \geq t_{r}(e)$ do

(10) send antimessage $\bar{e}$

(11) $C O Q \leftarrow C O Q-\{\bar{e}\}$ endfor end.

Figure 3.5: The cluster has received event $e$

\section{Inputs}

$e$ is the event to be sent.

\section{begin}

(1) send event $e$ to the destination cluster

(2) create $\bar{e}$ the antimessage of $e$

(3) $C O Q \leftarrow C O Q+\{\bar{e}\}$

end.

Figure 3.6: An LP passes to the CE event $e$ to be sent 


\subsection{Example}

\subsubsection{Receiving messages}

Figure 3.7a shows the space-time graph at a cluster composed of three logical processes. The $\mathrm{x}$-axis represents the virtual time and the $\mathrm{y}$-axis represents the location of the three LPs. Figure 3.7b shows the arrival of message $m_{1}$, whose receive time is 7 and whose destination process is $L P_{1}$. Since $m_{1}$ has been sent by an LP located in a different cluster. the Cluster Environment creates a new timezone starting at $\bar{T}$ which is indicated by the vertical line. Prior to the arrival of $m_{1}$, the cluster had only one timezone with interval $\left[0,+\infty\left[\right.\right.$. When $m_{1}$ has been received by the cluster, there exist two new timezones with intervals $[0,7[$ and $[\bar{\gamma},+\infty[$.
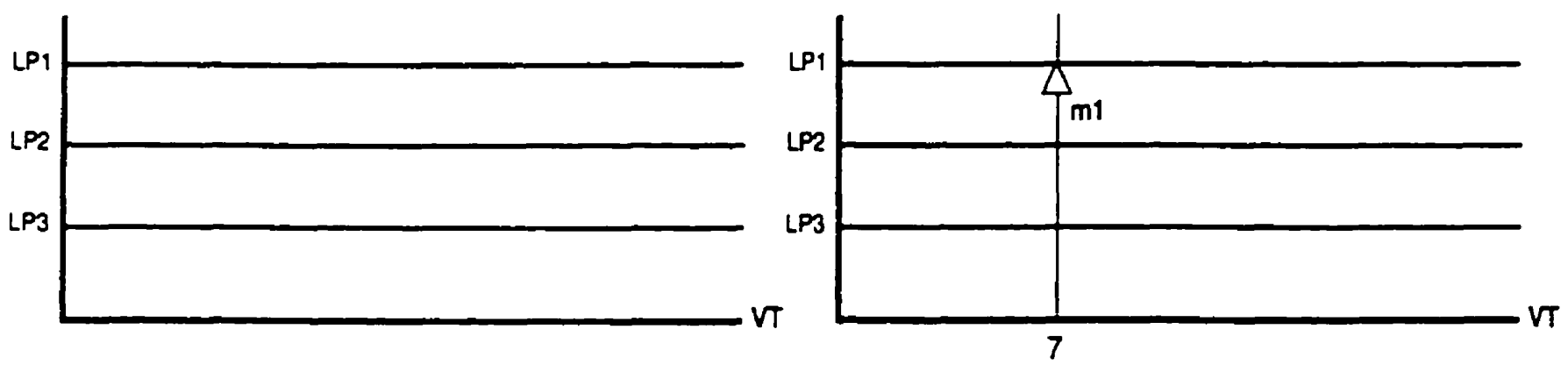

Figure 3.7: (a) The system starts and (b) message $m_{1}$ is received for $L P_{1}$

\subsubsection{Processing messages}

Now $L P_{1}$ is scheduled to process $m_{1}$. Since $m_{1}$ is located in timezone $\left[\tau_{+}+\infty\left[\right.\right.$ and $L P_{1}$ is in timezone [0.7[, the process performs a checkpoint and saves its state. The checkpoint is represented by the circle in figure 3.8. Then, the process advances its local clock to the value of the receive time of $m_{1}$ (indicated by the bold horizontal bar) and $L P_{1}$ processes $m_{1}$. A black triangle indicates that the message has been consumed, while a white triangle shows an unprocessed message. $L P_{1}$ is now in timezone $\left[\bar{\tau},+\infty\left[\right.\right.$. The processing of $m_{1}$ triggers the sending by $L P_{1}$ of messages $m_{2}$ and $m_{3}$ with receive times 9 and 11 and whose destination processes are $L P_{2}$ and $L P_{3}$ respectively. Since these two messages were generated within the cluster. no new timezone is created. 

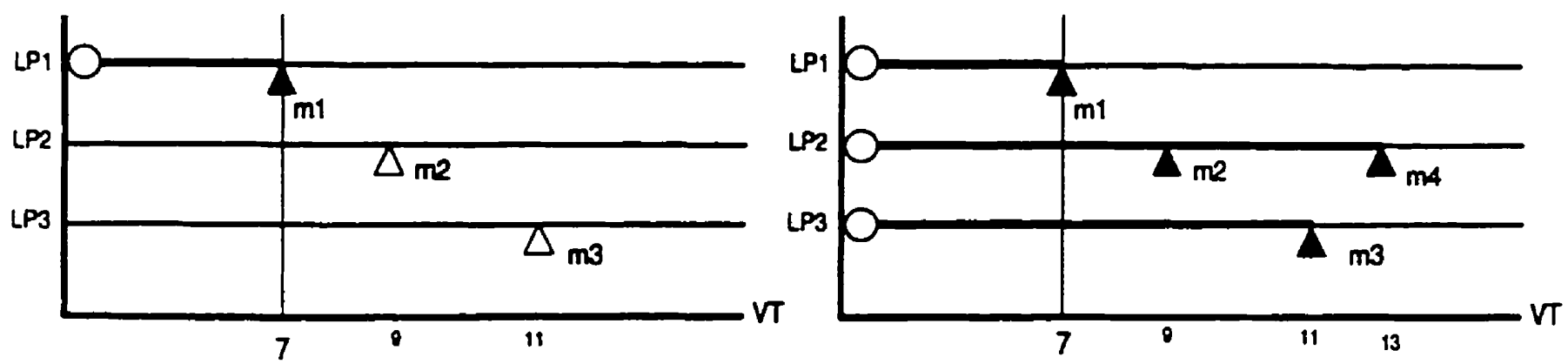

Figure 3.8: (a) $L P_{1}$ processes $m_{1}$ and (b) $L P_{2}$ and $L P_{3}$ process $m 2 . m 3$ and $m 4$

$L P_{2}$ is now scheduled to process $m_{2}$ since the receive time of this message is smaller than that of $m_{3}$. Like $L P_{1}, L P_{2}$ saves its state before entering a new timezone, advances its local clock. and processes $m_{2}$. Similarly. $L P_{3}$ is scheduled in its turn, its state is saved and $m_{3}$ is consumed. This triggers the sending of a new message $m_{4}$ whose destination process is $L P_{2}$ and receive time is 13. All of the LPs are now in timezone $\left[\bar{\imath},+\infty\left[\right.\right.$. Note that message $m_{4}$ generated by $L P_{3}$ did not create any new timezone because both the sending and the receiving process are located in the same cluster. Such messages are referred to as internal messages. Similarly. messages sent between clusters are referred to as external messages. $L P_{2}$ is now scheduled to process $m_{4}$, but since $m_{4}$ is located in the same timezone $\left[\bar{\tau}_{1}+\infty\left[\right.\right.$ as $L P_{2}$, the process does not save its state and directly consumes $m_{4}$ (figure $3.8 \mathrm{~b}$ ).

\subsubsection{Rolling back}
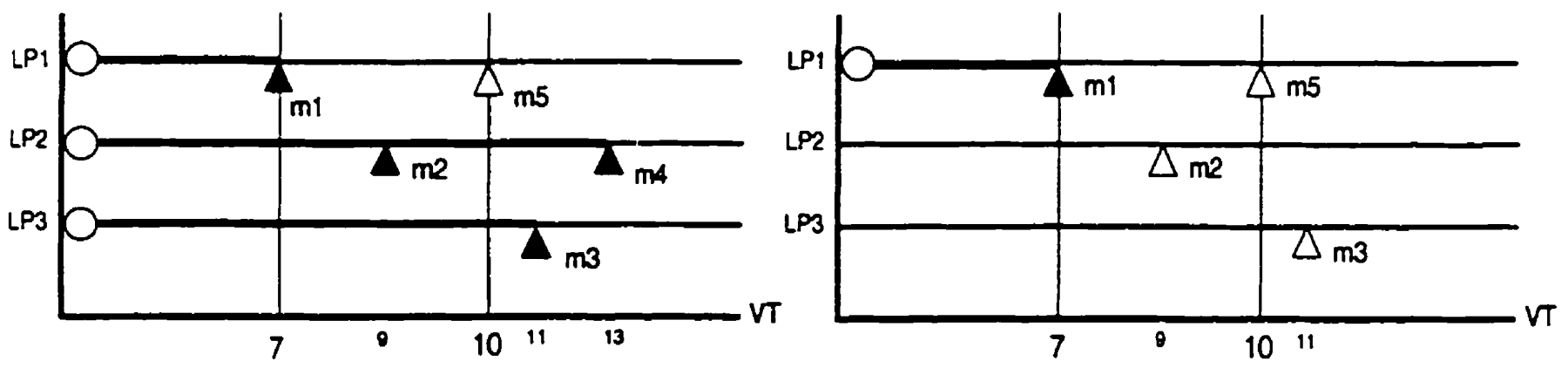

Figure 3.9: (a) $L P_{1}$ receives straggler $m_{5}$ and (b) $L P_{2}$ and $L P_{3}$ are rolled back and $m_{4}$ is discarded 
Suppose now that the cluster receives message $m_{5}$ with receive time 10 and whose destination process is $L P_{1}$. Since $m_{5}$ is an external message, the cluster splits timezone $[\bar{\tau},+\infty[$ into two new timezones with intervals $\left[7,10\left[\right.\right.$ and $\left[10,+\infty\left[\right.\right.$. As figure 3.9 a indicates. $L P_{2}$ and $L P_{3}$ have already processed messages with a timestamp larger than that of $m_{5}$ (which makes $m_{5}$ a straggler). In order to preserve the correctness of the system, $L P_{2}$ and $L P_{3}$ are both rolled back to a state prior to the receive time of $m_{5}$. Note that $L P_{1}$ does not need to be rolled back since it did not process any message with a timestamp larger than that of straggler $m_{5}$. After rolling back the processes. all the internal messages with a sending time larger than the receive time of the straggler are discarded since they will be regenerated if necessary by the rolled back LPs. In the example. $m_{4}$ has already been removed from the input queue since it has been processed by $L P_{2}$. Figure $3.9 \mathrm{~b}$ shows the state of the cluster once the straggler $m_{5}$ has been received, $L P_{2}$ and $L P_{3}$ are rolled back, and $m_{4}$ has been discarded. Note that messages $m_{2}$ and $m_{3}$ have now been marked as not having been processed. The cluster now contains three timezones with intervals $[0 . \bar{\tau}[,[\bar{\tau}, 10[$, and $[10,+\infty[$.
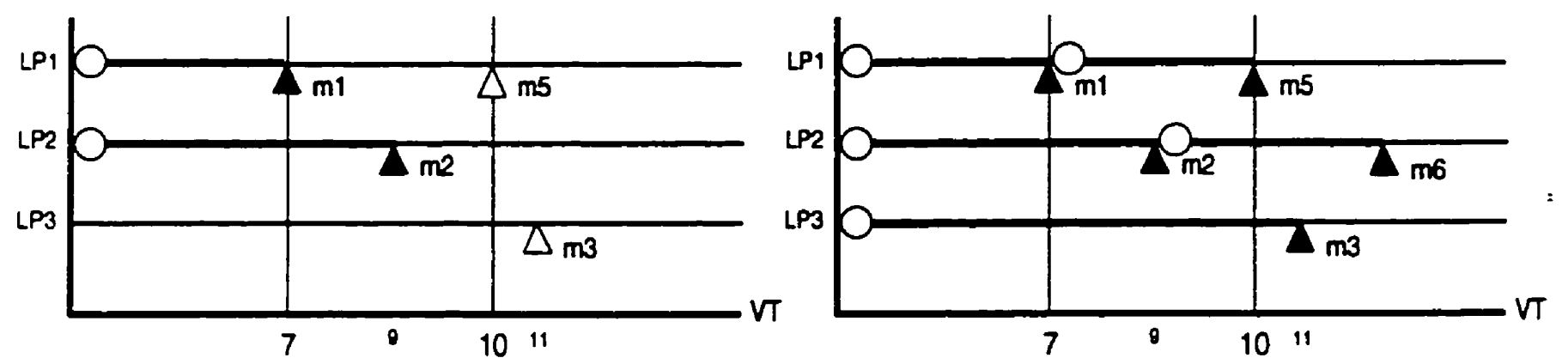

Figure 3.10: (a) $L P_{2}$ coasts forward and (b) the cluster resumes and proceeds normally

$L P_{2}$ can now coast forward resaving its state and reprocessing $m_{2}$. As for $L P_{3}$, it does not need to coast forward since it does not have any event to process with a timestamp smaller than that of the straggler $m_{5}$. Figure $3.10 \mathrm{a}$ shows the state of the cluster once $L P_{2}$ has completed the coast forward operation. The cluster can now resume to its normal behavior by scheduling $L P_{\mathbf{i}}$ to process $m_{5}$. Since $L P_{1}$ is going to enter a new timezone, its state is saved (figure $3.10 \mathrm{~b}$ ).

$L P_{3}$ is then scheduled next, saves its state before entering the new timezone $[10,+\infty[$, processes $m_{3}$ and sends $m_{6}$ to $L P_{2}$. Note that $L P_{3}$ skipped directly timezone $[\tau, 10[$ and did not perform a 
second checkpoint since it would have been useless as no message are being processed by $L P_{3}$ in that timezone. Finally, $L P_{2}$ processes $m_{6}$ after saving its state before entering timezone $[10 .+\infty[$.

\subsubsection{Antimessages}
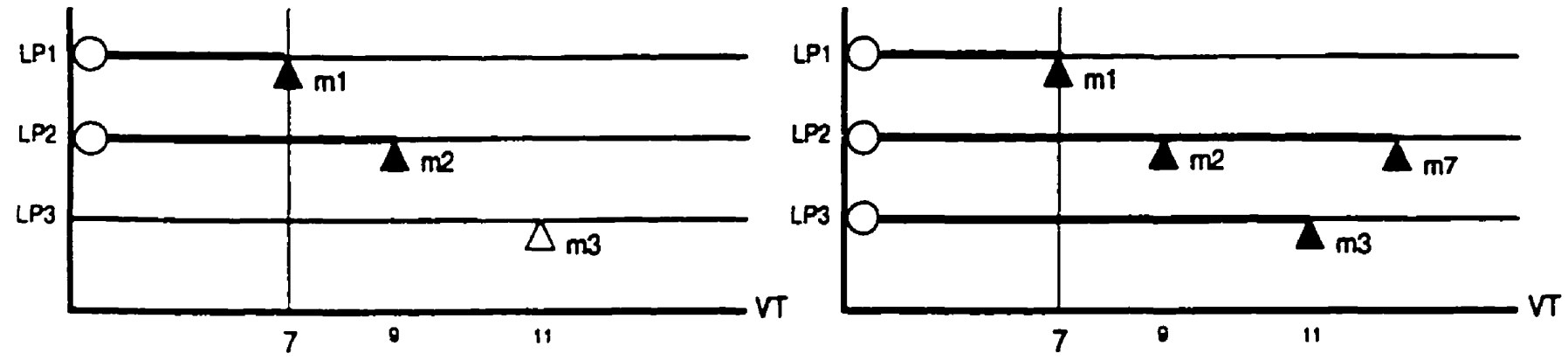

Figure 3.11: (a) $m_{5}$ is annihilated by its antimessage, the cluster rolls back, and (b) $m_{3}$ is reprocessed

Consider that the cluster is in a state as depicted by figure $3.10 \mathrm{~b}$ and receives $\overline{m_{5}}$. the antimessage of $m_{5}$. All LPs which have processed a message with a timestamp larger or equal to the timestamp of $m_{5}$ are then rolled back to a state prior to $m_{5}$. Message $m_{5}$ is now removed from the input queue and the two timezones [ $\bar{\imath} .10[$ and $[10,+\infty[$ are merged into one single timezone $[\bar{\tau} .+\infty[$ as there is no more external input messages located in that interval. Figure 3.11 a shows the state of the cluster once all LPs have been rolled back and message $m_{5}$ has been annihilated. The cluster resumes and $L P_{3}$ is now scheduled to process $m_{3}$ which causes $m_{7}$ to be generated and sent to $L P_{2}$. Finally: $L P_{2}$ processes $m_{i}$ (figure $3.11 \mathrm{~b}$ ).

\subsection{Estimating the GVT}

Our fossil collection algorithm differs somewhat from that of Time Warp. In the Clustered Time Warp approach. the state prior to the GVT must be saved. while in Time Warp this is not necessary: The reason for this is that it is possible to roll back to a point prior to the GVT because not every event is checkpointed. Similarly, the events prior to the GVT in the LP input queue cannot all be removed. As it is possible for the LP to rollback to a state prior to the GVT. 
events with timestamp smaller than the GVT might have to be reprocessed while the LP coasts forward. Once an estimate of the GVT has been calculated, all the LPs can discard the states prior to the GVT but one, and preferably, the one whose timestamp is the closest to the GVT. Then, all the events whose receive time is smaller than the timestamp of the oldest state can be also discarded. Figure 3.12 shows the pseudocode executed by each logical process when a new GVT estimate has been calculated.

\section{Input}

$C I Q$ is the Cluster Input Queue.

$C O Q$ is the Cluster Output Queue.

GI $T$ is the new estimated GVT value.

$\Psi$ is the state queue of the logical process.

\section{begin}

(1) let $\Psi_{\text {old }} \subseteq \Psi \mid \forall S_{i} \in \Psi_{\text {old }} \Rightarrow T L E\left(S_{i}\right) \leq G V T$

(2) select state $S_{0} \in \Psi_{\text {odd }} \mid T L E\left(S_{0}\right)=\operatorname{Max}\left(T L E\left(S_{i}\right)\right) \forall S_{i} \in \Psi_{\text {odd }}$

(3) $\Psi_{\text {old }} \leftarrow \Psi_{\text {old }}-S_{0}$

(4) for each state $S_{i} \in \Psi_{\text {old }}$ do

(5) $\Psi \leftarrow \Psi-\left\{S_{i}\right\}$ endfor

(6) for each message $e_{i} \in C I Q \mid t_{r}\left(e_{i}\right)<T L E\left(S_{0}\right)$ do

(i) $C I Q \leftarrow C I Q-\left\{e_{i}\right\}$ endfor

(8) for each message $e_{j} \in C O Q \mid t_{s}\left(e_{j}\right)<T L E\left(S_{0}\right)$ do

(9) $C O Q \leftarrow C O Q-\{e$, endfor

end.

Figure 3.12: Operations performed by the LP once a new GVT estimate is calculated

In the current implementation of Clustered Time-Warp. a token-ring passing algorithm [Prei89] is used since the architecture used to develop the system (the BBN Butterfly) does not contain a large number of nodes (maximum of 32 nodes). Furthermore, even though the memory of the machine is physically distributed. the shared-memory paradigm guarantees atomic message delivery. Had the system been implemented on the top of a communication network. an extra mechanism should have been developed to ensure that no message is hidden in a communication 
channel during the GVT calculation (see chapter 1).

\subsection{Space-based Checkpointing Techniques}

All existing dynamic checkpointing techniques (see chapter 1) are time-based since logical processes choose to change the checkpoint interval based on their rollback history. The new checkpointing technique that naturally results from the Clustered Time Warp approach described previously is the first space-based checkpointing technique. In other words, the checkpoint interval of an LP depends on the origin of the message received, and not on the rollback history of the process. In this section. we introduce two other variants of the original checkpointing technique developed for Clustered Time Warp.

\subsubsection{Clustered Rollback, Clustered Checkpoint}

In the Clustered Time Warp algorithm, when a straggler or an antimessage arrives at the cluster. all of the LPs which have processed an event with a receive time larger than that of the straggler or of the antimessage will be rolled back. The decision to rollback is therefore taken at the cluster level, thus we define this technique as clustered rollback.

Checkpointing is performed each time an LP changes timezone. Since timezones are dynamically created by the Cluster Environment depending upon the arrival of messages coming from other clusters, we denote this mechanism as clustered checkpoint.

Clustered Rollback - Clustered Checkpoint (CRCC) is the rollback and checkpointing technique that naturally results from the Clustered Time Warp approach.

This technique has the advantage of reducing memory consumption by discarding all of the messages in invalidated timezones as they will be regenerated. However. the expense of forcing these LPs to roll back each time an antimessage or a straggler arrives at the cluster is not negligible, especially if most of the events generated by the LPs within that cluster are not causally related to the event which caused the rollback. In such a case. only a few LPs actually need to be rolled back. 


\subsubsection{Local Rollback, Clustered Checkpoint}

Since there is a risk to waste computational resources in CRCC due to the fact that all the LPs in a cluster are rolled back even if it is not necessary for them to do so, a compromise was sought in which the decision of rolling back is made by the logical process itself.

In this new scheme, when a straggler or an antimessage is received by the cluster. the Cluster Environment updates the timezone table accordingly and places the event into the input queue of the receiving LP. LPs now behave much as they do in a pure Time Warp system: rolling back when they detect the arrival of a straggler in their input queue and sending antimessages when needed. Hence. logical processes also need an output queue to keep track of the messages they send in order to cancel wrong computations in the case they have to roll back. As a direct consequence. the cluster does not need to have an input queue nor an output queue. therefore. the CIQ and the COQ can be discarded, and the Cluster Environment ends up only taking care of updating the timezone table when external events come into the cluster.

This technique is called Local Rollback - Clustered Checkpoint (LRCC) since the decision to roll back is made at the LP level. and checkpointing is still performed at the cluster level via the timezone table.

Although this scheme might offer less overhead in terms of computation. it is more expensive in terms of memory since all the events in the LP input queue as well as those in the LP output queue have to be kept as they will not be regenerated.

\subsubsection{Local Rollback, Local Checkpoint}

In this variant of Clustered Time-Warp. an LP checkpoints only if it receives an external message. in other words a message that has been generated by another LP located in a different cluster. This scheme is simpler in the sense that LPs do no longer need to check whether they are entering a new timezone. Furthermore. the Cluster Environment does not need anymore to maintain a timezone table. Hence. compared to the other techniques described above, this scheme requires the least computational overhead.

Because the decisions of rolling back and checkpointing are both performed at the LP level. this technique is called Local Rollback - Local Checkpoint (LRLC). 
Even though it is evident that an LP will have fewer checkpoints compared to the schemes described earlier, it is not obvious at all it will save more memory. On the contrary, and although it appears counter-intuitive, this scheme can be more greedy. Since the distance between checkpoints is greater, the number of events an LP needs to keep (in order to coast forward if it rolls back to a state prior to the GVT) tends to grow. Therefore, there is a trade-off: the fewer states an LP saves, the more events it needs to keep. In the case of logic simulation, the size of an event is far from being negligible compared to that of a state. Therefore the distance between checkpoints should not grow excessively if we want to keep the usage of the memory to a minimum.

\subsection{Summary}

We presented Clustered Time Warp. a novel hybrid algorithm that takes advantage of the characteristics of logic simulation and which can be implemented on general purpose parallel architectures.

This new approach offers several adiantages over Time Warp:

- First. CTW requires less memory than Time Warp since logical processes do not save their state each time they process a message. Also, fewer copies of messages need to be kept since all LPs are rolled back when a cluster receives a straggler (internal events whose timestamp is larger than that of the straggler are automatically regenerated if necessary). Furthermore. CTW might perform even better than other state-skipping checkpointing techniques since it takes advantage of the intrinsic structure of the circuit.

- Another advantage of the Clustered Time Warp approach when the CRCC checkpointing technique is used is that incorrect computations are cancelled more rapidly than in a pure Time Warp environment. When a straggler or an antimessage arrives at a cluster. antimessages are sent immediately to other clusters since all the LPs are bypassed. In the case of Time Warp, the cancellation wave needs to propagate through all the logical processes, which takes much more time. Furthermore, if that cancellation wave does not quickly catch 
up the computation wave, the risk of rollback explosions increases significantly. We study this phenomenon in chapter 7 .

- Finally, the fact that LPs are already grouped into clusters gives an edge to the algorithm for the implementation of a dynamic load-balancing mechanism. By considering groups of processes, the size of the problem can be reduced by one or several orders of magnitude depending upon the size of the cluster. Consequently, balancing the load becomes simpler and is likely to also be more effective because heuristics can be gathered at the cluster level. Furthermore, the implementation also becomes simpler since the routing tables need only be associated with clusters rather than with LPs. On the whole. a large amount of computational and memory overhead can be saved.

We have described the first family of space-based checkpointing techniques for parallel simulation using an optimistic approach. These checkpointing algorithms are much simpler to implement than optimal checkpointing algorithms and each of them occupies a different point in the spectrum of possible trade-offs between memory usage and execution time (see figure 3.13).

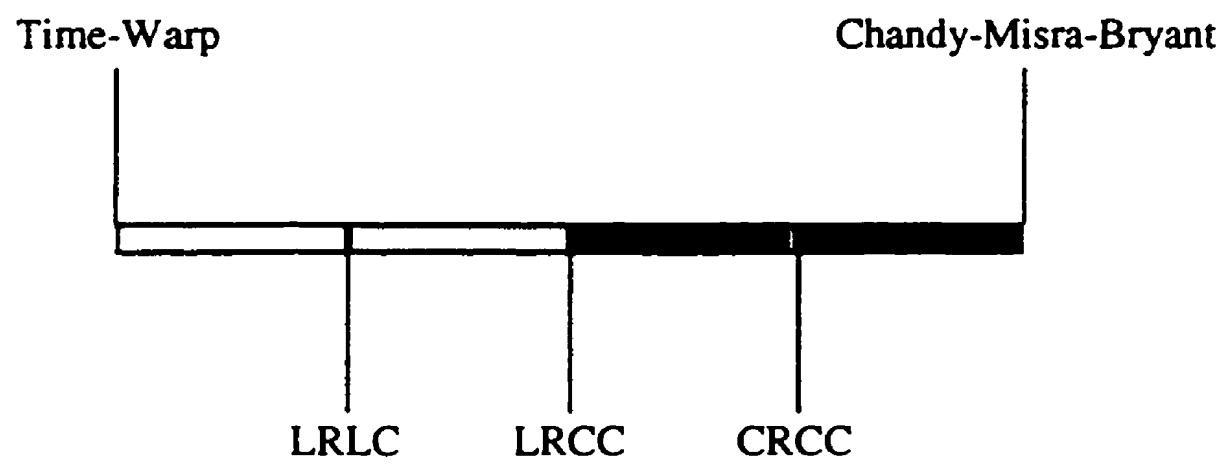

Figure 3.13: Checkpointing techniques

Clustered Rollback - Clustered Checkpoint (CRCC) is the most conservative technique and requires less memory than the two other checkpointing techniques. Nevertheless. the fact that all processes in a cluster are rolled back could reduce the performance of the system. Local Rollback - Local Checkpoint ( $L R L C$ ) is the technique that is the closest to a pure Time Warp system and it should therefore perform fairly well in terms of execution time. However, the price to pay will be memory. Local Rollback - Clustered Checkpoint (LRCC) is midway between 
LRLC and CRCC and should consequently give performance results between LRLC and CRCC in terms of both execution time and memory consumption.

Regarding the scalability problem of Time Warp, the CRCC technique can significantly reduce the risk of rollback explosions observed in which the cancellation of wrong computations is unable to catch up with the computation [Luba89]. Since logical processes decide locally whether or not to rollback in both the LRCC and the LRLC techniques, the risk of rollback explosions is therefore as high as in Time Warp. 


\section{Chapter 4}

\section{Experiments and Results}

\subsection{The Multiprocessor Environment}

In this chapter, we evaluate the performance of Clustered Time Warp and its different checkpointing techniques introduced in chapter 3 . Our algorithm is compared to pure Time Warp and a variant of Time Warp using periodic state saving.

We used a BBI Butterfly GP1000 shared-memory multiprocessor (see figure 4.1) for our experiments. The Butterfy is an MIMD machine composed of 32 processor nodes. Each node has an MC68020 and MC68881 processors with 4 megabytes of memory and a high-speed multistage crossbar switch which interconnects the processors. From a processor point of view, remote and local memory references are identical. thus creating a global virtually shared memory space. The crossbar switch is a banyan network composed of $4 \mathrm{x} 4$ switch elements and is interfaced with each node by an $A .12901$ microprocessor whose purpose is to ensure the atomicity of memory operations performed on remote references.

The BBN Butterfly platform runs the Mach operating system and supports TotalView', a source-level. window-oriented. multiprocessor debugger that provides a collection of powerful tools for the development of parallel and distributed applications.

An asynchronous message passing layer was implemented on the top of the shared memory so that the results obtained from running the different algorithms are not dependent on the presence of shared variables. hence making any comparisons unfair. Furthermore. a future port of the simulator to distributed memory architectures will be made easier. The message passing

\footnotetext{
${ }^{1}$ Totalview is a trademark of BBN Systems and Technologies
} 


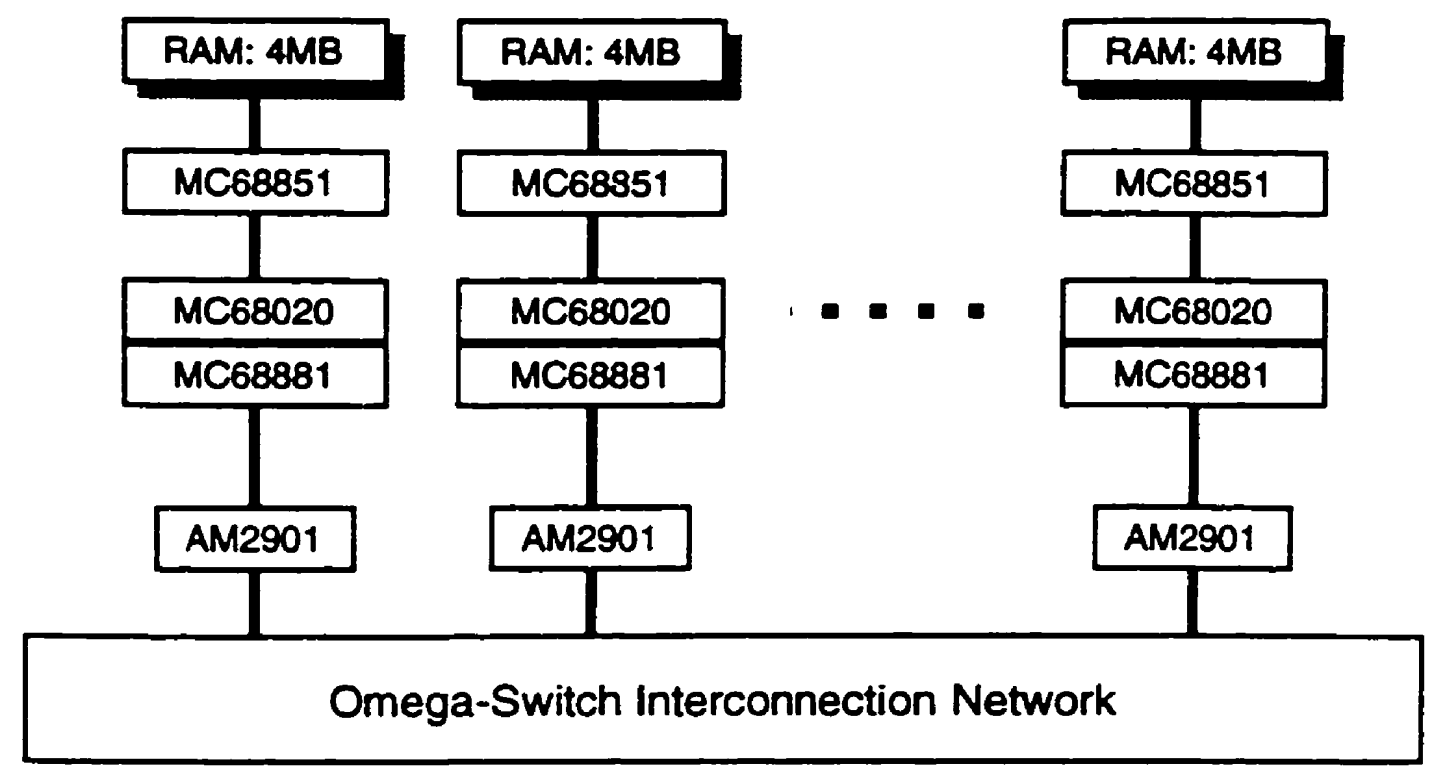

Figure 4.1: The GP1000 shared-memory multiprocessor

layer provides two non blocking communication primitives: send() and receive(). Furthermore. messages can either have a low or a high priority. If a high priority message is awaiting. it is delivered to the processor before any other low priority message, regardless of their arrival time. Otherwise. if no high priority message is awaiting. low priority messages are delivered to the processor in the order they were received.

Table 4.1 compares the execution times of arithmetic operations executed on the BBN Butterfly (MC68000 processor) and the Intel iPSC/860 (Intel Personal SuperComputer/860). The iPSC/860 is a distributed memory architecture in which the processors are connected in a hypercube topology: Each node has 8 megabytes of memory and a 32 bit RISC processor running at $40 \mathrm{MHz}$. Table 4.2 shows the transmission time of messages between two nodes for both the BB. Butterfly and the iPSC/860 [Bouk95]. The BBN Butterfly is several years older than the iPSC/860. consequently, there exists a difference in terms of performance. More importantly, these data show that the iPSC/860 is about twice as fast as the BBN Butterfly for both arithmetic operations and message transmissions. For instance, on the iPSC/860. long integer additions are about 2.2 times faster. Similarly, sending a 100 byte message is about 2.65 times faster ${ }^{2}$. This suggests that the performance results obtained on the BBN platform will also apply to a dis-

\footnotetext{
${ }^{2}$ Our logic simulator does not use float operation and the size of the messages is fairly small
} 
tributed memory architecture such as the Intel iPSC/860. If our simulation system had used the shared memory paradigm of the BBN Butterfly to share data structures such as event queues or process states, extrapolation of performance results to distributed memory architectures would have been too hypothetical.

\begin{tabular}{|c|c|c|}
\hline \multirow[t]{2}{*}{ Operation } & \multicolumn{2}{|c|}{ Execution time $(\mu \mathrm{s})$} \\
\hline & $\overline{B B N}$ Butterfly & iPSC $/ 860$ \\
\hline short integer + & 1.35 & $1.3 \bar{T}$ \\
\hline long integer + & 2.33 & 1.07 \\
\hline short integer ${ }^{*}$ & 2.98 & 1.69 \\
\hline long integer ${ }^{*}$ & 5.05 & 1.44 \\
\hline float + & 8.82 & 5.58 \\
\hline float * & 8.82 & 6.32 \\
\hline double + & 10.99 & 6.64 \\
\hline double * & 10.99 & 8.52 \\
\hline
\end{tabular}

Table 4.1: Arithmetic Operation Times ( $\mu \mathrm{s})$

\begin{tabular}{|c|c|c|}
\hline \multirow{2}{*}{$\begin{array}{c}\text { Message Length } \\
\text { (bytes) }\end{array}$} & \multicolumn{2}{|c|}{ Transmission time (ms) } \\
\cline { 2 - 3 } & BBN Butterfly & iPSC/860 \\
\hline 0 & 1.35 & 0.6 \\
100 & 2.12 & 0.8 \\
512 & 3.36 & - \\
1024 & 6.29 & 2.1 \\
4096 & 30.9 & 4.3 \\
\hline
\end{tabular}

Table 4.2: Message Transmission time between nodes

\subsection{Simulation System}

As discussed in chapter 1, we oriented our algorithms towards the simulation of logic-level VLSI circuits. Like most simulators, our logic simulation model uses three discrete logic values: 1. 0 and undefined. To model the propagation delay, each gate has a constant service time. All of the common logical gates were implemented: AND, NAND. OR. NOR, XOR. XNOR, NOT. and D-type flip-flops. 


\begin{tabular}{|c|c|c|c|c|c|c|}
\hline name & \# inputs & \# outputs & \# flip-flops & total & \multicolumn{2}{|c|}{ Asynchronous Parallelism } \\
\cline { 5 - 6 } & & & & & Average & Relative \\
\hline s35932 & 35 & 320 & 1,728 & 18,148 & 1,839 & $10.13 \%$ \\
\hline s38584 & 12 & 278 & 1,452 & 20,995 & 1,107 & $5.27 \%$ \\
\hline
\end{tabular}

Table 4.3: Circuit s35932 and s38584

The circuits used in our study are digital sequential circuits selected from the ISCAS' 89 Benchmarks. We present the results obtained from simulations of two of the largest circuits (table 4.3) since they are both representative of the results we obtained with the other circuits and have different characteristics. For example. circuit s38584 has a relative asynchronous parallelism

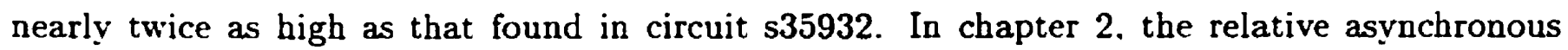
parallelism was defined as being the average number of events an asynchronous algorithm can process concurrently divided by the total number of gates in the simulated circuit.

A program was written to read the netlist of the ISCAS benchmark circuits and to partition them into clusters. We used a string partitioning algorithm, because of its simplicity and especially because results have shown that it favors concurrency over cone partitioning; see for example [Brin91]. The algorithm is similar to an inorder tree walk [Corm91]. A gate connected to a primary input is first selected and assigned to a cluster. Its output is then followed and the same procedure is applied for each succeeding gate. When the cluster contains the desired number of gates. a new cluster is created and the algorithm resumes. Figure 4.2 shows a potential string assignment for circuit $s 27$ for a cluster size of 4.

A simulation run can be decomposed into three phases. First, each processor starts up by loading the gates assigned to it and by creating their corresponding LPs. Then. each gate which has an initialized state produces an event to the gates connected to it. Some of these gates will be triggered and will propagate their changes throughout the circuit. After a while the system becomes stable, and events stop being generated. During the third phase, input vectors (previously randomly generated) are read and the simulation is run. Once the termination of the system is detected, statistics are collected. 


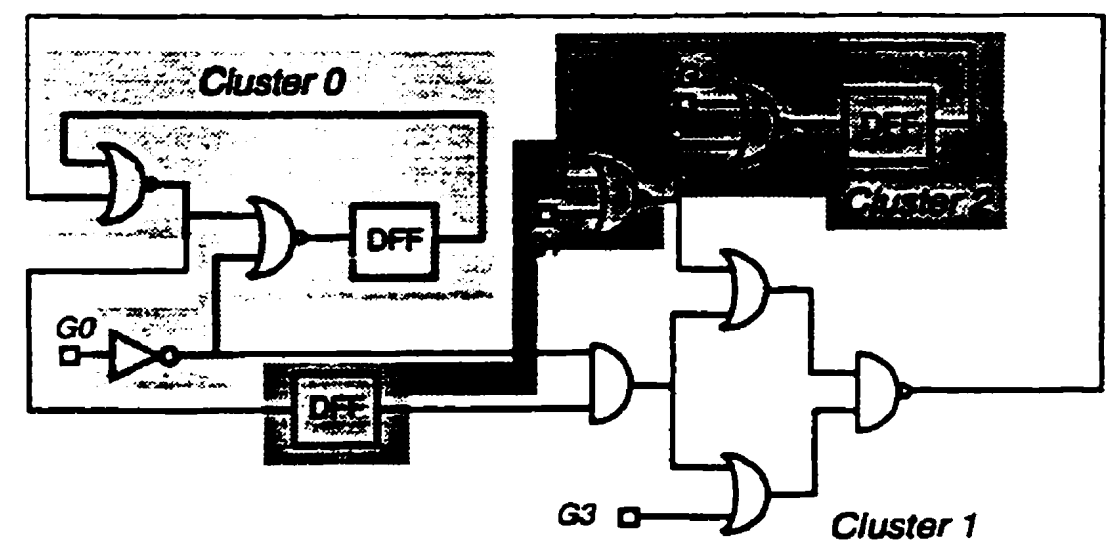

Figure 4.2: Example of a string partitioning for circuit s2 7 (cluster size $=4$ )

\subsection{Experiments}

We conducted two categories of experiments: one was to determine the effects of cluster size on the performance of each algorithm. and a second set of experiments to compare the performance (memory and execution time) of the algorithms with that of Time Warp. Because previous studies [Brin90. Reih90] have shown that lazy cancellation does not actually perform better, we used an aggressive cancellation strateg in all our experiments. For each simulation run, three metrics were used to evaluate the performance of the algorithms: the simulation time, the peak number of states and the peak memory usage.

\section{Simulation Time}

We define $\tau$ to be the simulation time such that: $\tau=t_{n}-t_{0}$ where $t_{0}$ and $t_{n}$ are the real time at which respectively the first and the last event were processed by the system. $\tau$ is expressed in seconds.

\section{Peak Number of States}

During a simulation run, process $L P_{i}$ constantly monitors the size of its state queue $\Psi_{L P_{i}}$. Let $\psi_{L P_{1}}(t)=\left|\Psi_{L P_{8}}(t)\right|$ be the size of $\Psi_{L P_{1}}$ at real time $t$ such that $0 \leq t \leq \tau$. We define the number of states of processor $P_{k}$ at real time $t$ to be $\psi_{P_{k}}(t)=\sum \psi_{L P_{i}}(t) \forall L P_{i} \in P_{k}$. Let the peak number 
of states of processor $P_{k}$ be $\hat{\psi}_{P k}=\operatorname{Max}\left(\psi_{P_{k}}(t)\right)$ where $0 \leq t \leq \tau$. We define the peak number of states of a simulation as:

$$
\hat{\psi}=\operatorname{Max}\left(\psi_{P_{k}}\right) \quad \forall P_{k} \in \Pi
$$

where $\Pi$ is the set of processors involved in the simulation. The peak number of states is therefore the maximum number of states required by any host during the entire simulation.

\section{Peak Memory Usage}

In addition to $\Psi_{L P_{i}}, L P_{i}$ also monitors the size of both its input event queue $\Omega_{L P_{1}}^{i n}$ and its output event queue $\Omega_{L P_{i}}^{\text {out }}$. Let $\omega_{L P_{i}}(t)=\left|\Omega_{L P_{i}}^{\text {in }}(t)\right|+\left|\Omega_{L P_{i}}^{\text {out }}(t)\right|$ be the number of events stored in $\Omega_{L P_{1}}^{\text {in }}$ and $\Omega_{L P_{1}}^{\text {out }}$ at real time $t$. Furthermore, each cluster $C_{j}$ monitors the size of both its input queue

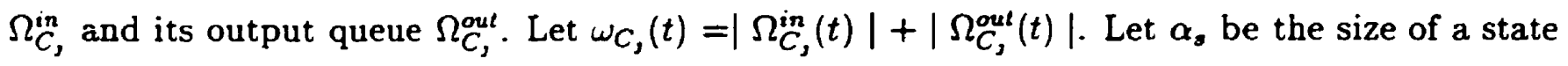
and $\alpha_{e}$ the size of an event. We define the memory usage of a processor $P_{k}$ at real time $t$ as:

$$
\alpha_{P_{k}}(t)=\sum_{\forall L P_{i} \in P_{k}}\left(\alpha_{s} \cdot \psi_{L P_{1}}(t)+\alpha_{e} \cdot \omega_{L P_{i}}(t)\right)+\alpha_{e} \cdot \sum_{\forall C, \in P_{k}} \omega_{C_{j}}(t)
$$

Note that when the CRCC checkpointing technique is used $\Omega_{L P_{1}}^{\text {out }}=\emptyset$ since LPs do not need an output queue. Similarly, $\Omega_{C,}^{\text {in }}=\Omega_{C}^{\text {out }}=\emptyset$ for the other techniques since there is no cluster output queue and no cluster input queue.

Let the peak memory usage of processor $P_{k}$ be $\hat{\alpha}_{P_{k}}=\operatorname{Max}\left(\alpha_{P_{k}}(t)\right)$ where $0 \leq t \leq \tau$. We define the peak memory usage of a simulation as:

$$
\hat{\alpha}=\operatorname{Max}\left(\alpha_{P_{k}}\right) \quad \forall P_{k} \in \Pi
$$

where $\Pi$ is the set of processors involved in the simulation. The peak memory usage is therefore the maximum memory required by any host during the entire simulation and is only dependent on the number of states and the number of events stored in memory.

\subsubsection{Varying the cluster size}

In this category of experiments. we ran a series of circuit simulations for each algorithm on a fixed number of processors (20). The only parameter that was changed during the tests was the 
size of the clusters. In the first run, the size was such that all of the processors hosted only one cluster. In the second run, there were 2 clusters per processor, 4 in the third test, so on until a maximum of 256 clusters per processor was reached.

\section{Peak memory usage}

Figure 4.3 shows the peak memory usage in kilobytes vs. the number of clusters per processor for circuit s35932. The graph indicates a rather stable behavior on the part of LRCC and LRLC with a minimal memory usage occurring at 2 clusters per processor. At this point LRCC needs $38 \%$ less of the memory than pure Time Warp to run the simulation, and LRLC $22 \%$ less.

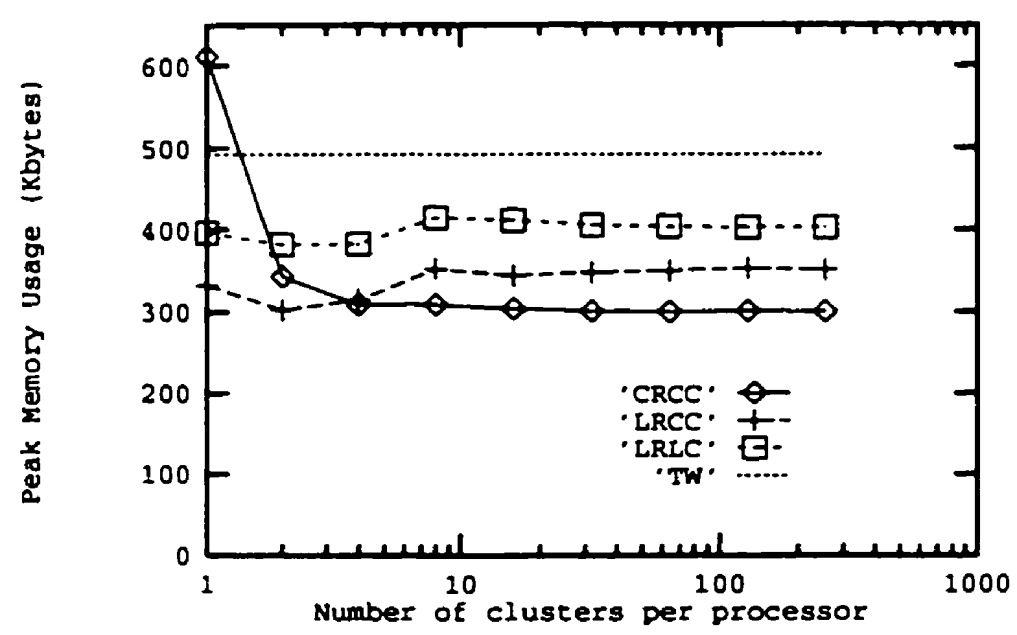

Figure 4.3: Memory vs. Number of clusters per processor (circuit s35932)

As for CRCC. we observe a rather high memory usage when each processor contains only one cluster. This is indirectly due to the synchronization overhead incurred by the algorithm itself. When a straggler is received by a cluster, all the processes whose TLE is greater than the receive time of that straggler have to be rolled back. This operation is expensive since one straggler can roll back several hundreds of processes, even though most of these processes are not causally related to that straggler. This will have the effect of desynchronizing the LPs, thus increasing the risk of rollbacks in other processors. This problem suddenly disappears when 2 clusters per processor are used. In this case, the cluster size is halved and the effect of a straggler becomes less 
dramatic. The memory usage for the CRCC checkpointing technique decreases until 4 clusters per processor, at which point it becomes constant. The data show up to a $40 \%$ difference in maximal memory usage between CRCC and Time Warp.

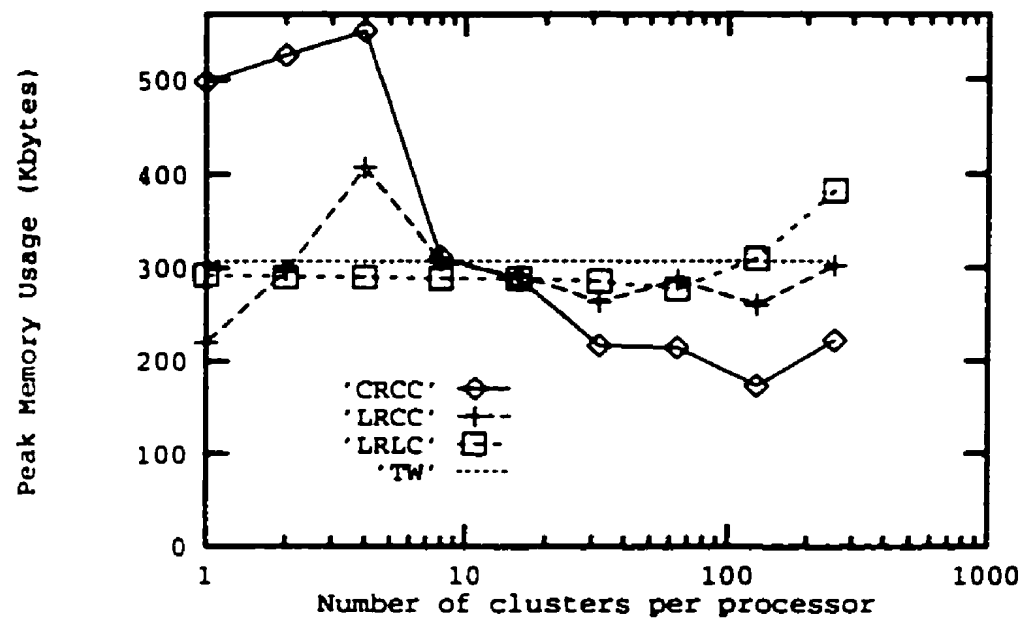

Figure 4.4: Memory vs. Number of clusters per processor (circuit s38584)

Figure 4.4 shows the peak memory usage for circuit $s 38584$. On the whole, all the checkpointing techniques of Clustered Time llarp do not perform as well as in the previous case. For example. LRLC requires between 5 to $10 \%$ less memory than Time Warp and LRLC needs about 4 to 15\% less memory: As for CRCC. the memory consumption is rather high from 1 to 4 clusters per processor. After that point. the memory usage drops down to reach a minimal value at 128 clusters per processors where the memory requirements are about $43 \%$ smaller than Time Warp.

The difference in the peak memory consumption between the two circuits is due to to the fact that circuit s38584 has a relative asynchronous parallelism nearly half that of circuit s35932 (see table 4.3). This characteristic of circuit 538584 has two consequences. First. because fewer events are being processed in parallel. the Clustered Time Warp approach has a smaller chance to take advantage of its sparse checkpointing techniques. Take for example an LP that receives only one event between two GI'T computations. In such a case it does not really matter what the checkpoint interval is. since the LP will have to perform at least one checkpoint anyway. Thus, if we consider a simulation in which LPs process very few events. the overall memory usage of any checkpointing technique will not be very important. 
In addition, when a circuit having a small parallelism is simulated, the event population in the system is likely to be relatively small too, hence reducing the number of process states that have to be saved. Because less objects are being manipulated by the system. the estimated GVT tends to be closer to the actual GVT, therefore the fossil collection mechanism is able to remove most of the useless states and events. As a direct consequence, the memory usage reduction that can be achieved by Clustered Time Warp is attenuated.

\section{Simulation Time}

Figures 4.5 and 4.6 show the simulation time vs. the number of clusters per host. We observe that CRCC has a significant overhead when compared to Time Warp. This is mainly due to the fact that some LPs are unnecessarily rolled back. Also, each time a cluster receives a straggler or an antimessage. the cluster has to check all of its LPs to find out whether or not they have to be rolled back. This overhead becomes more pronounced when the cluster size is large. From 64 clusters per processor and onward, the simulation time for CRCC becomes constant and is about $34 \%$ higher than that obtained with pure Time Warp.

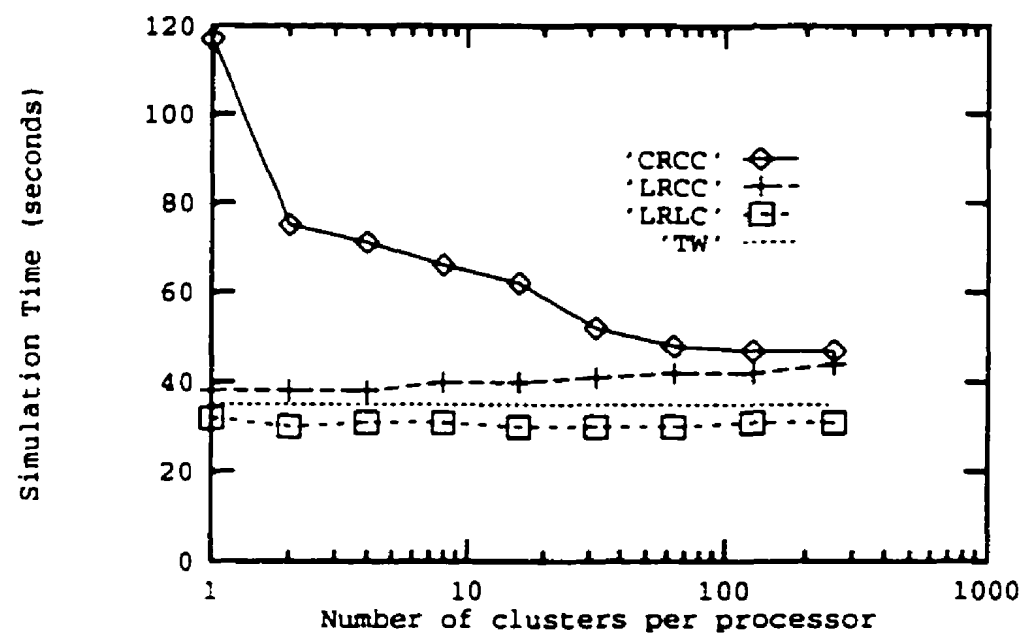

Figure 4.5: Simulation time vs. Number of clusters per processor (circuit s35932)

For both LRCC and LRLC, the simulation time is approximately constant for any cluster size. LRCC is about $10 \%$ slower than pure Time Warp since clusters need to update their timezone 
table regularly, and because LPs check the table each time they are about to process an event. As for LRLC, it is about 5 to $15 \%$ faster than Time Warp because fewer states are saved. Consequently, the fossil collection mechanism has less work to do and can catch up quickly.

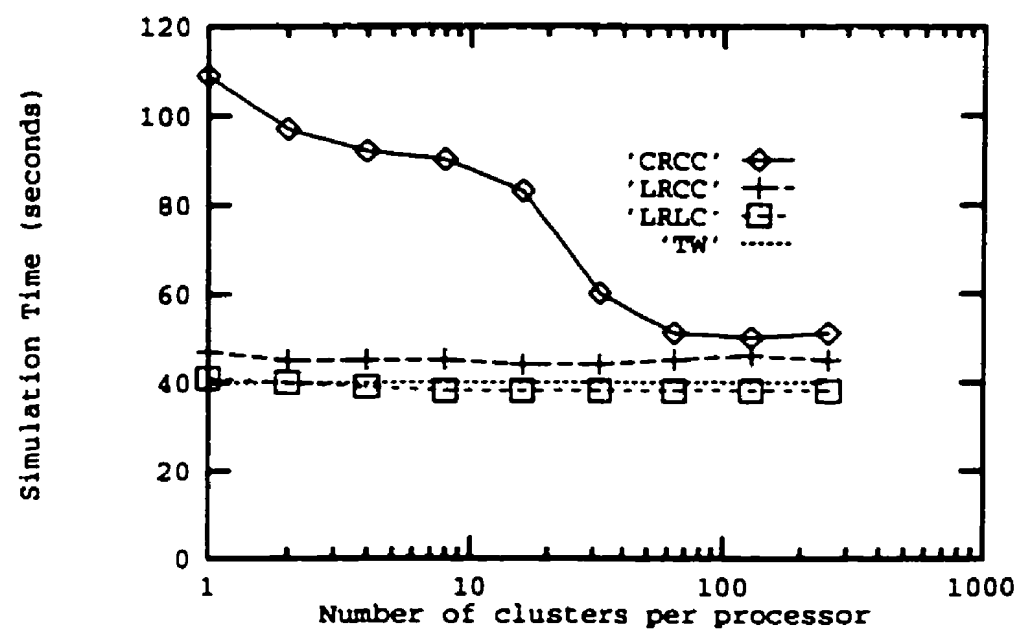

Figure 4.6: Simulation time vs. Number of clusters per processor (circuit s38584)

Relative to Time Warp. the fact that LRCC performs slightly better for circuit s38584 and LRLC performs better for circuit s35932 is again a direct consequence of the parallelism available in the circuit. LRCC is slower than Time Warp because of the overhead created by the timezone management. A smaller parallelism implies a smaller overhead. thus better performance. Similarly. LRLC is faster than Time Warp because the checkpoint interval is sparse and the overhead due to the garbage collection mechanism is reduced. However, if the parallelism gets small, the event population becomes small too, and less fossil objects have to be collected. Therefore, the reduction of the garbage collection overhead is less significant.

\section{Summary}

Based on these results, we chose the cluster size for each algorithm which gave the best performance in order to use them in our second set of experiments. For LRCC and LRLC, we chose one cluster per processor. In the case of CRCC, we chose 32 and 128 clusters per processor for circuits s35932 and s38584 respectively. 


\subsubsection{Varying the number of processors}

In the second set of experiments we observed the behavior of the algorithms, varying the number of processors from 8 to 24 . In addition we also show the performance of a Periodic State Saving mechanism (PSS) which is a modified version of pure Time Warp in which the checkpoint interval is constant and larger than one. In our study, we chose a checkpoint interval of 3 as it proved to be an optimal value for a large range of type of simulations [Prei94].

\section{Peak Number of states}

The main reason why checkpointing techniques are used in optimistic algorithms is the reduction of the memory usage. Nevertheless. no study has so far demonstrated that a larger checkpoint interval results necessarily in a smaller memory usage. Figure 4.7 shows an example of two logical processes $L P_{1}$ and $L P_{2}$ whose checkpoint intervals are 3 and 2 respectively. Triangles represent events and circles represent checkpoints. Suppose a new GVT estimate is calculated and both LPs are about to collect their fossil objects. In addition to the state prior to the GVT. LPs need to keep all of the succeeding events in order to be able to restore their state during the coast forward phase of rollback recovery. For this reason. $L P_{1}$ does not actually have any fossil object whereas $L P_{2}$ can delete 2 fossil events and 1 fossil state. Consequently, even though $L P_{1}$ has a larger checkpoint interval. its memory usage is larger than that of $L P_{2}$.

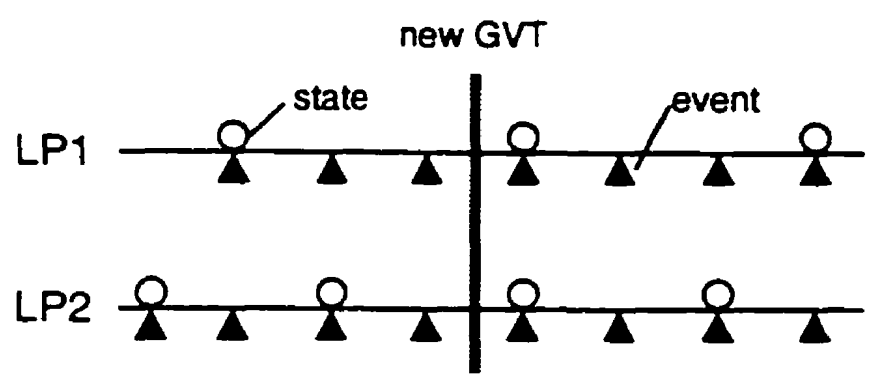

Before garbage collection

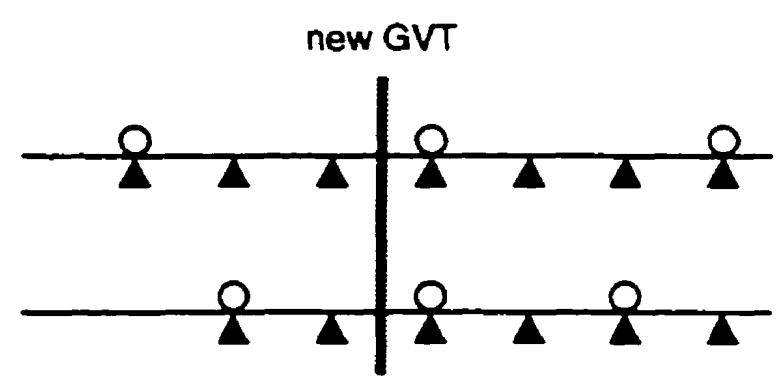

After garbage collection

Figure 4. $\bar{\tau}$ : Larger checkpoint interval does not always imply smaller memory usage

This problem is actually more important in the case of logic simulation where the event size is of the same order of the state size. If the distance between checkpoints becomes too large, 
the memory used to keep events (needed for the coast-forward phase) could become larger than the memory saved by skipping checkpoints, in which case the overall space performance of the algorithm might not be improved.

To illustrate this problem, we measured the peak memory usage used by each algorithm. as well as the peak number of states.

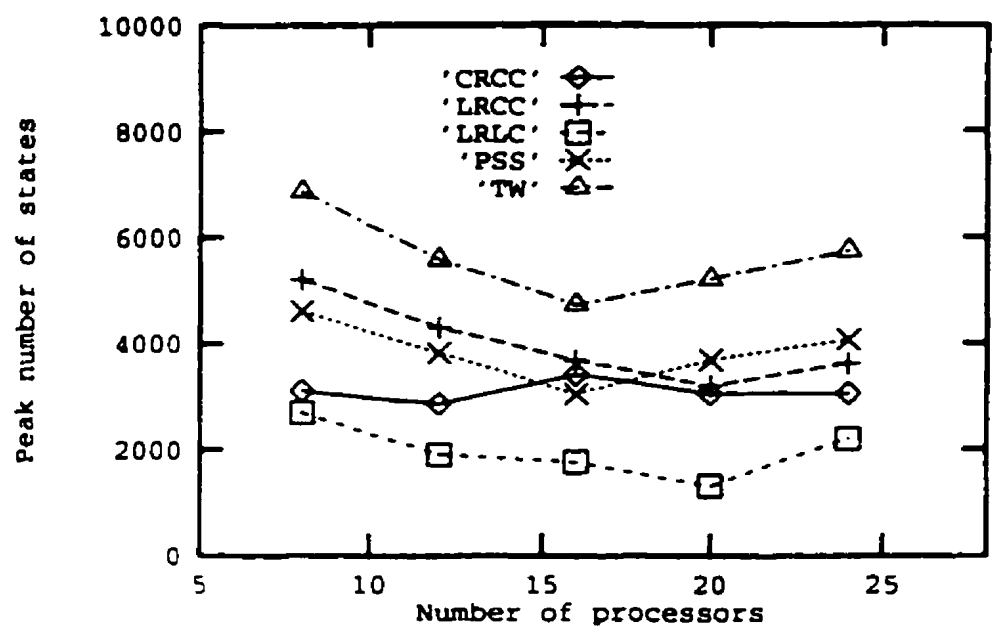

Figure 4.8: Number of states vs. Number of processors (circuit s35932)

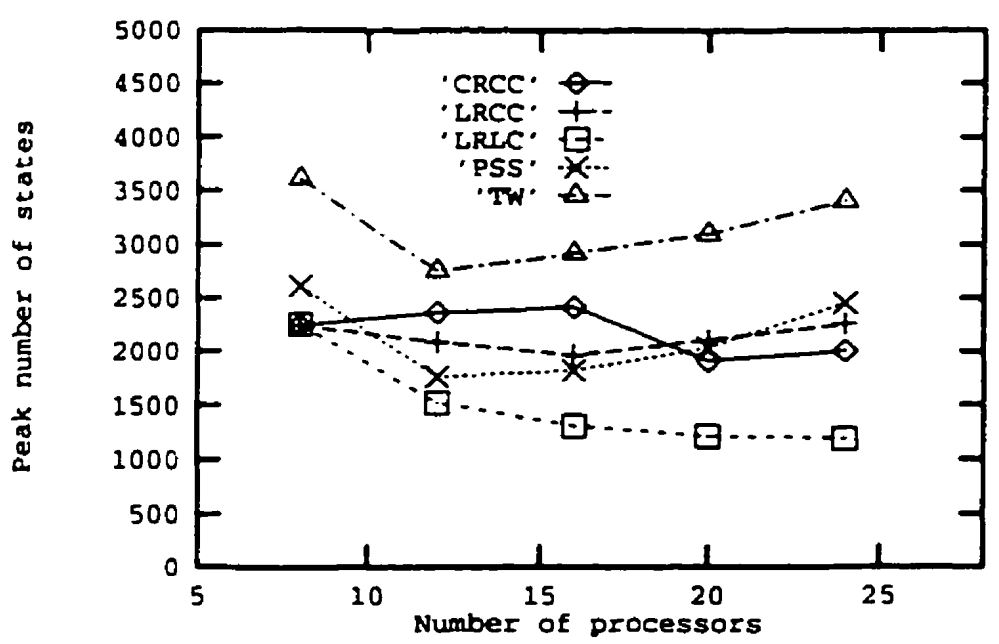

Figure 4.9: Number of states vs. Number of processors (circuit s38584) 
In figures 4.8 and 4.9 , we show the peak number of states for each algorithm vs. the number of processors for the circuits s35932 and s38584 respectively. For both circuits, and regardless of the number of processors, all algorithms require less state saving than Time Warp. However, the LRLC checkpointing technique is by far cheaper since it stores some $70 \%$ fewer states than Time Warp in some cases. CRCC, LRCC and PSS all use approximately 30 to $40 \%$ less states than Time Warp.

\section{Peak Memory Usage}

In figures 4.10 and 4.11 , we show the peak memory usage of each algorithm vs. the number of processors for circuits $\mathbf{3} 35932$ and s38584 respectively. In all cases, the proposed algorithms consume less memory than pure Time Warp.

The phenomenon we described previously can now observed. For circuit s35932, when compared to Time Warp, the CRCC checkpoint protocol, which saved half as many states as LRLC (see figure 4.8). actually performs much better than LRLC when all the memory usage is considered (see figure 4.10). Similarly, when compared to Time Warp, the periodic state saving technique with a checkpoint interval of 3 (PSS), saves only between 9 and $16 \%$ of the memory usage whereas it saved between 30 and $35 \%$ of the states.

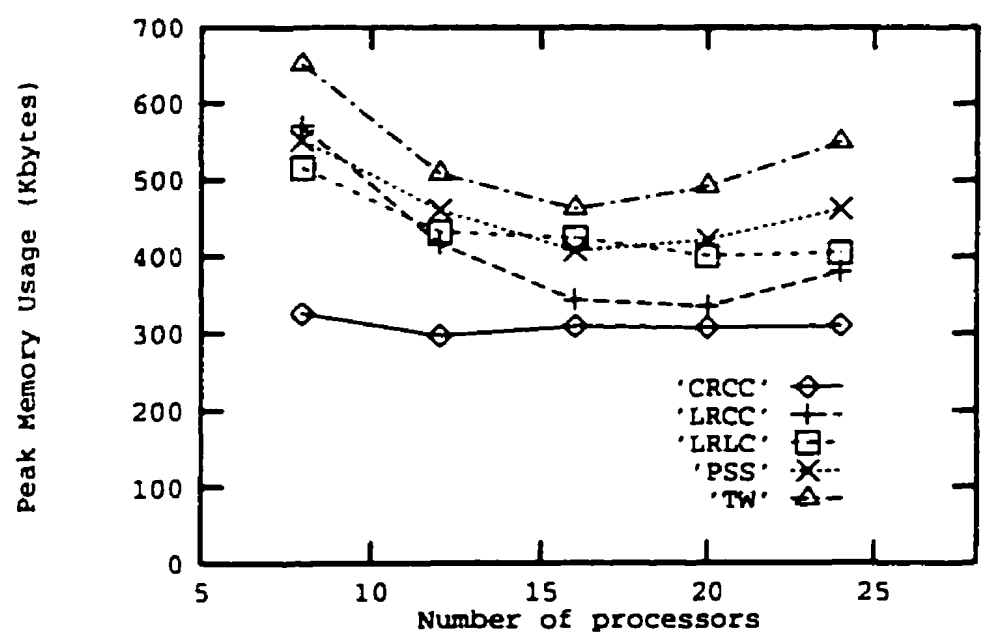

Figure 4.10: Memory usage vs. Number of processors (circuit s35932) 


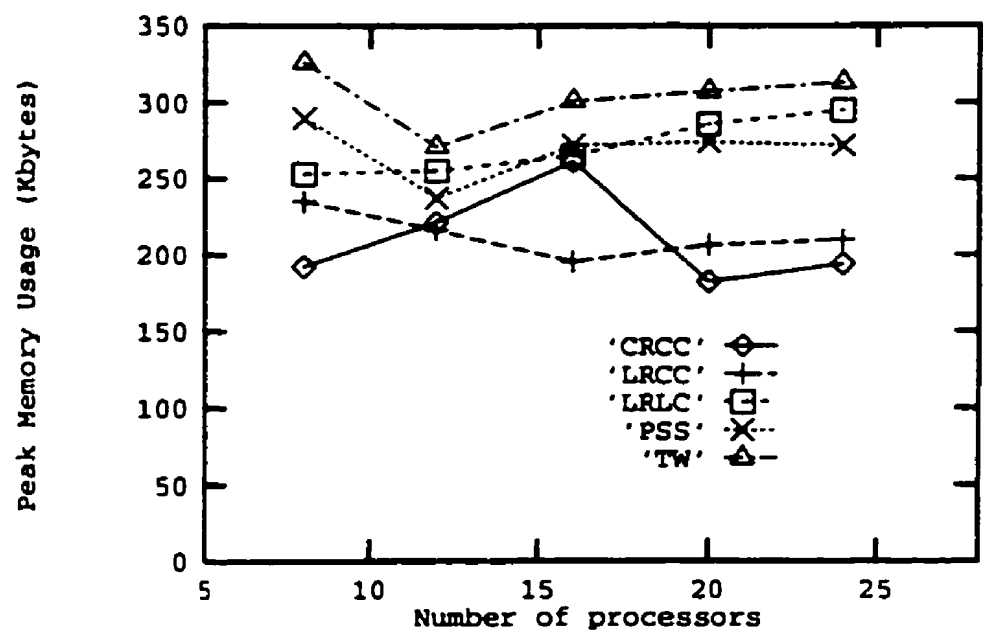

Figure 4.11: Memory usage vs. Number of processors (circuit s38584)

These results show the importance of taking events into consideration for the design of checkpointing techniques for optimistic algorithms.

The same phenomenon is observed for circuit $\mathbf{3 8 5 8 4}$ (figure 4.11). In this case. even though the activity of the circuit is much smaller than circuit s35932, the CRCC checkpoint protocol uses between 15 and $40 \%$ less memory than Time Warp depending on the number of processors being used. Also. despite the fact that the PSS protocol saved between 28 and $37 \%$ of the states: the total memory usage was actually reduced only by about 10 to $13 \%$.

\section{Simulation time}

In figures 4.12 and 4.13 . we present the simulation time of each algorithm vs. the number of processors. We observe that both LRCC and LRLC perform comparably to Time Warp. CRCC is from 30 to $60 \%$ slower than pure Time Warp in these examples. We note that this difference becomes less significant as the number of processors increase (since the memory is itself more distributed among the processors). 


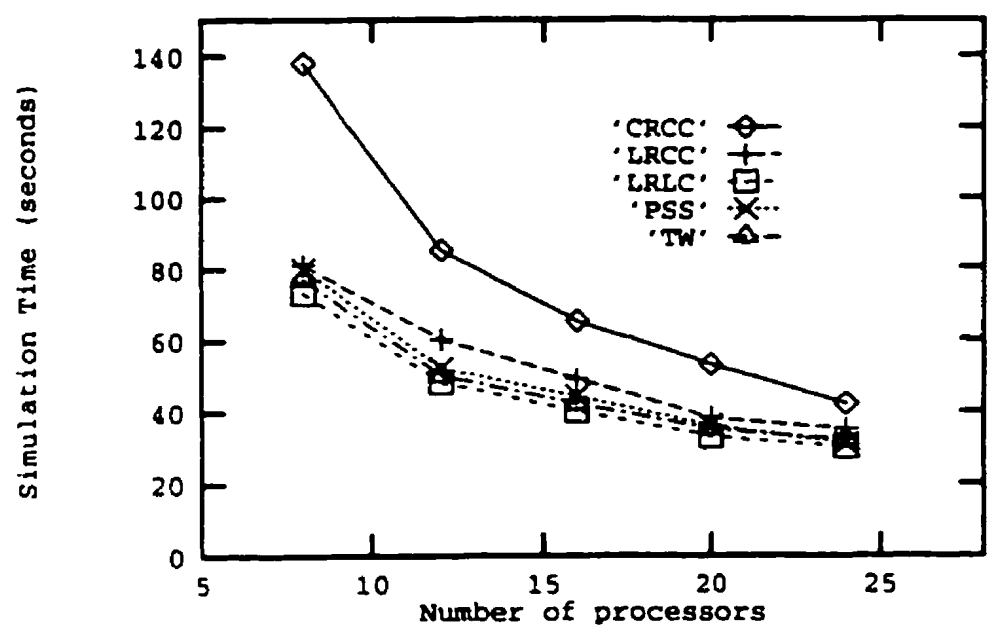

Figure 4.12: Simulation time vs. Number of processors (circuit s35932)

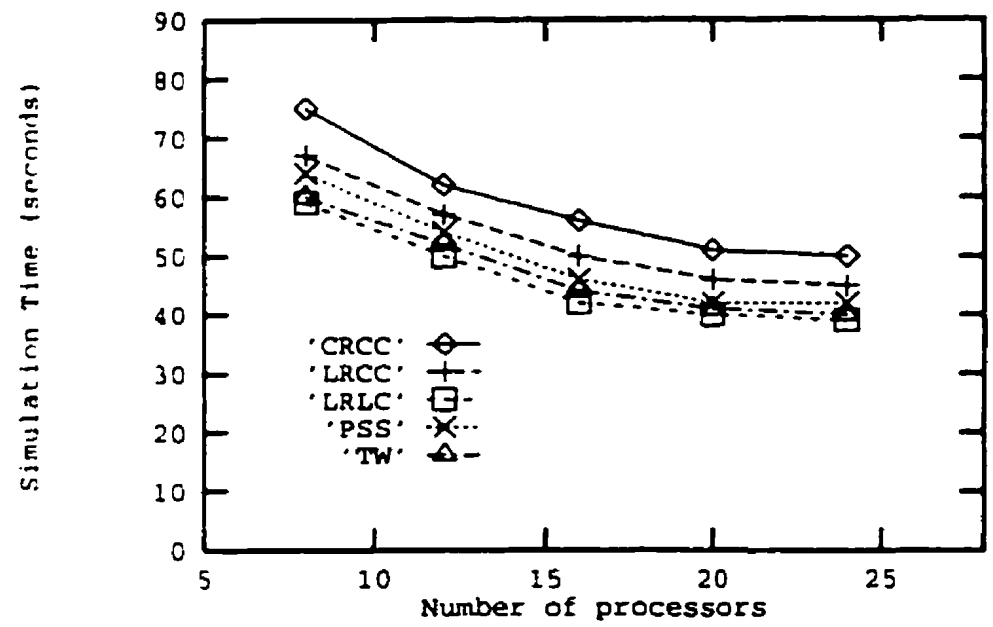

Figure 4.13: Simulation time vs. Number of processors (circuit s38584)

\subsubsection{Speedup}

In order to measure the speedup obtained with the parallel simulation system. we have developed a sequential simulator. In this case. since the simulation is performed on a single processor, there is no need for synchronization. therefore no checkpointing is performed and events are deleted as soon as they are processed. As a consequence. no GVT algorithm is needed and the fossil 


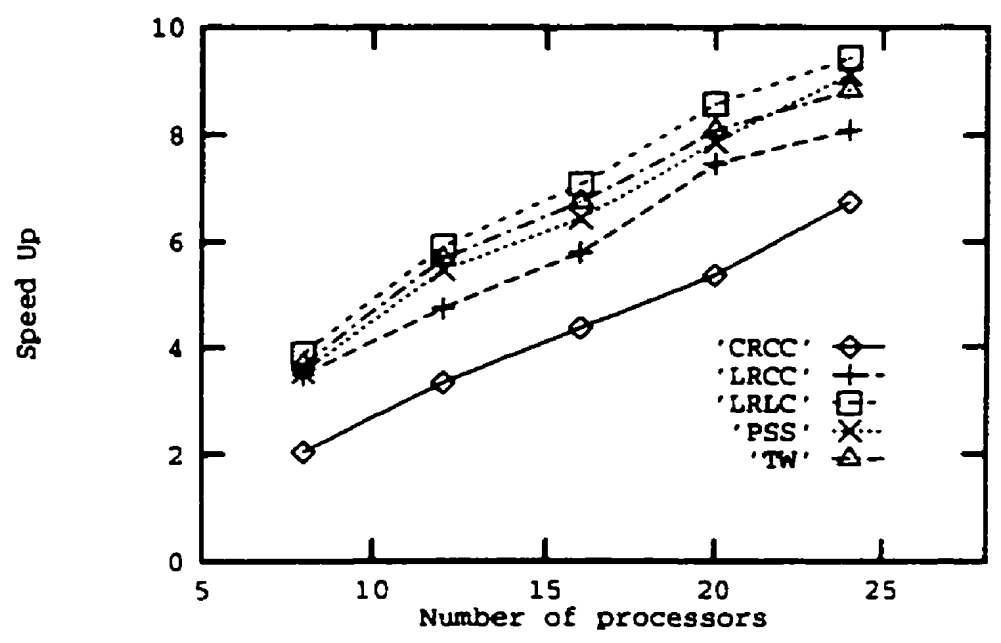

Figure 4.14: Speedup observed for circuit s35932

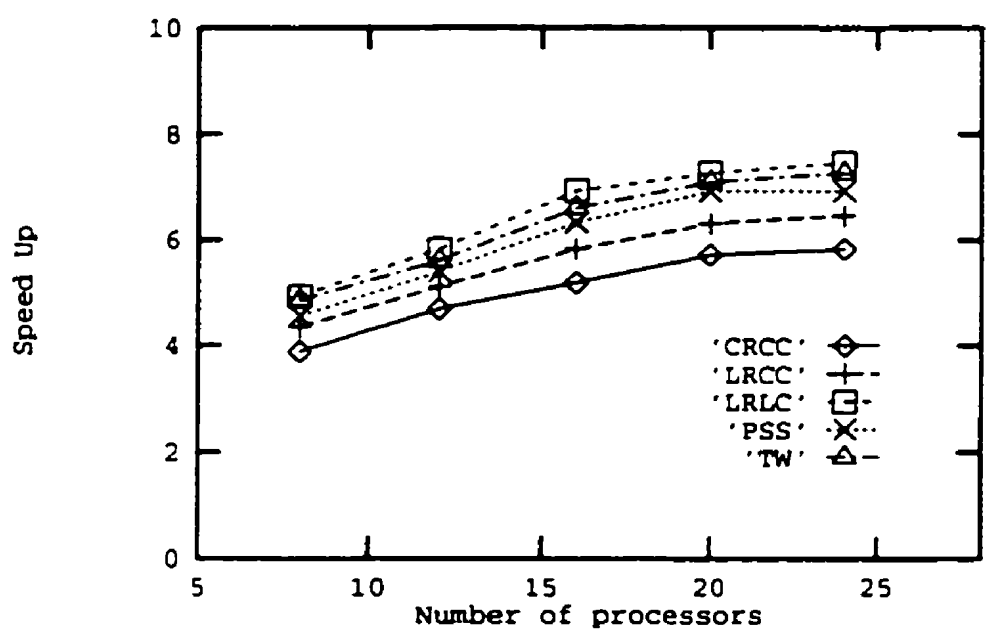

Figure 4.15: Speedup observed for circuit s38584

collection mechanism is simply switched off. The scheduling of the processes is performed with a single heap and a minimum message timestamp first policy is used. The sequential simulation for circuits s35932 and s38584 took 283 and 291 seconds respectively.

Results are shown in figures 4.14 and 4.15. As we have seen in table 4.3. the parallelism available in circuit $\mathbf{s} 35932$ is much higher than that available in circuit s38584 (the relative parallelism is twice as high), as a consequence, the speedup obtained from the parallel simulation of circuit s35932 is relatively higher than circuit s35932. When the number of processors is 
relatively small, the overhead of the synchronization algorithm becomes more significant, and we observe that the speedup is actually better for a circuit with less concurrency. This clearly shows that the performance of asynchronous algorithms depends highly on the intrinsic parallelism available in the simulated circuits, but also in the ability of these algorithms to keep their overhead relatively small.

\subsection{Summary}

Figures 4.16 and 4.17 summarize the results by comparing each algorithm with pure Time Warp for circuits s35932 and s38584 respectively. For each algorithm, we give the minimum, the maximum and the average percentage difference from pure Time Warp for the maximum number of states, the peak usage of memory, and the simulation time.

We first observed that each algorithm saves a substantial number of states, especially LRLC. However. these results do not necessarily directly translate into those obtained for total memory usage.

However when the clustered checkpointing mechanism of CTW is employed (ie: LRCC and $\mathrm{CRCC}$ ), the performance is better in terms of memory consumption. These results underline the fact that in simulation models such as logic simulation in which the size of the state of the LPs is approximately the same as the size of the events, it is important to consider the increase of memory needed to store the supplementary events due to the checkpoint interval.

As to the simulation time, only CRCC is much slower than pure Time Warp, whereas the other algorithms exhibited a speed comparable to Time Warp.

The results also point out a stable behavior of the algorithms with respect to the number of clusters employed. With this range of choices among checkpointing algorithms. it is possible to choose an algorithm depending upon the memory requirements of the simulation. 


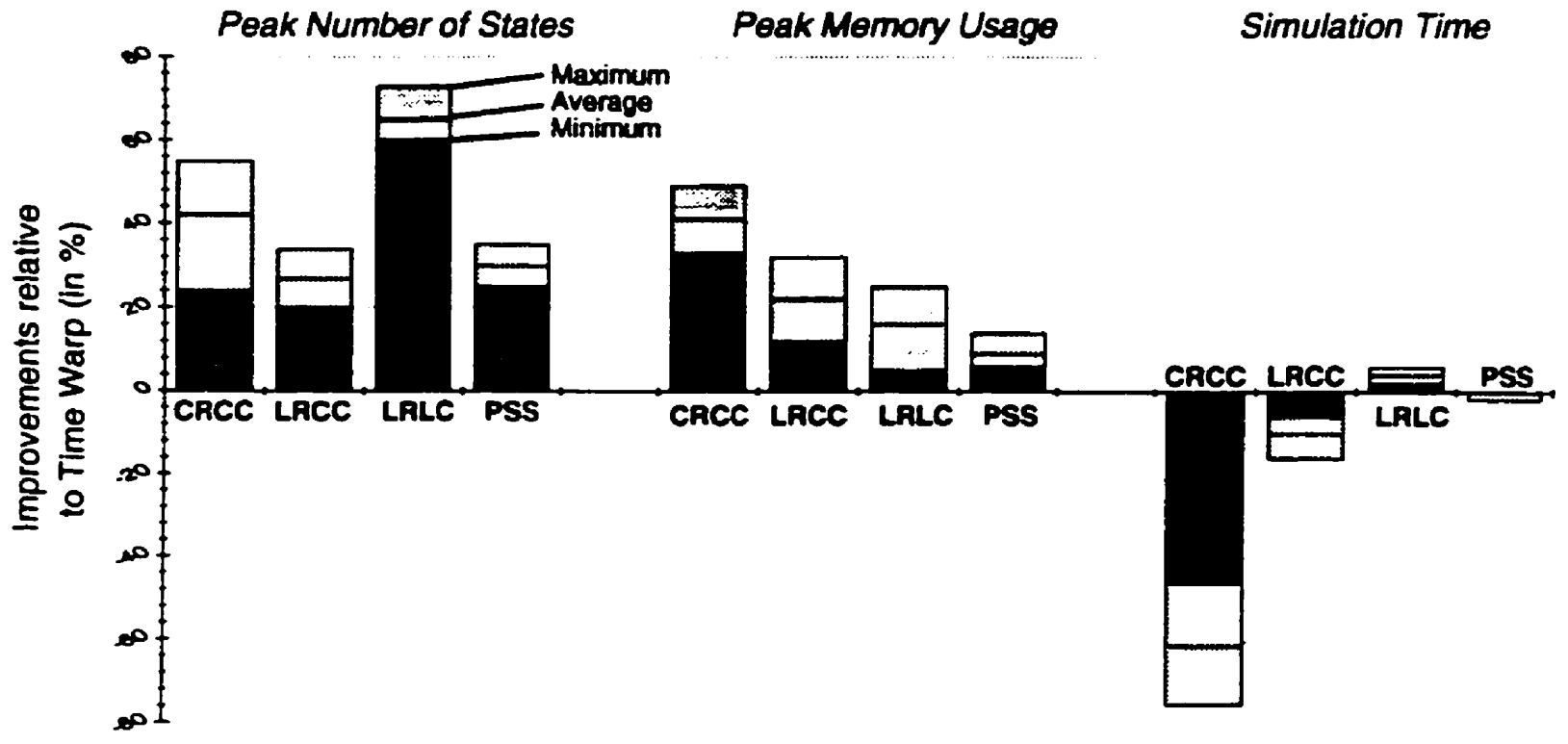

Figure 4.16: Space-Time performance results for circuit $\mathrm{s} 35932$

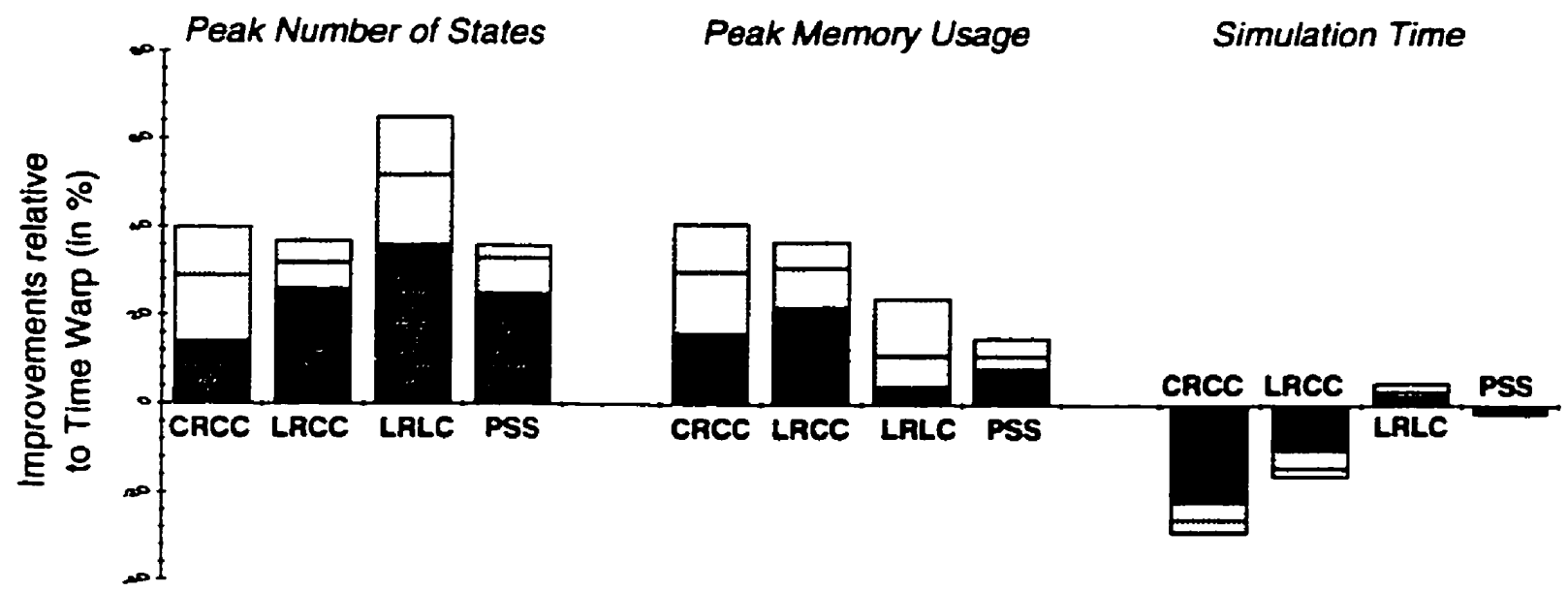

Figure 4.17: Space-Time performance results for circuit s38584 


\section{Chapter 5}

\section{Dynamic Load Balancing}

\subsection{Introduction}

Empirical results [Nob195] have shown that a very strong locality exists in digital circuits, suggesting that historical information can be used to improve the mapping of the simulated model onto processors. Furthermore. we have observed in chapter 2 that the locality of workload is relatively strong in digital circuits.

In this spirit. we feel that the use of a dynamic load balancing technique can substantially improve the performance of logic simulations. Furthermore, the fact that Clustered Time Warp groups LPs into clusters makes the load balancing mechanism easier to implement since instead of having to deal with individual LPs, only clusters are considered.

In this chapter, we describe a dynamic load balancing algorithm whose objective is to attempt to evenly distribute the load among the processors. Our results indicate that the most important factor in the load balancing of parallel VLSI simulations is keeping the processors as busy as possible. even at the expense of increased inter-processor communications and rollbacks.

\subsection{Related results}

In [Nico85], Nicol and Reynolds present a statistical approach to dynamically partition a circuit and to map it to a set of processors. In their approach, the circuit is pre-simulated and a work graph is created to describe the precedence relations between the nodes. Edges in the work graph are weighted according to the communication delays between the nodes. If over a clock cycle, the 
time region of the activity of two nodes overlap, parallelism can be achieved by mapping these two nodes on two different processors. Given the work graph, the authors partition the nodes into clusters by using a min-cut clustering algorithm based on Losen's approach [Lose85]. The complexity of their algorithm is $O\left(E \cdot(N-K) \cdot \log _{2}(N-K)\right)$ where $N$ is the number of nodes in the work graph, $E$ is the number of edges and $K$ is the number of partitions. Their empirical results are positive. Nevertheiess, the tests were done on a single 64-gate circuit and the authors assumed networks having no directed cycles. Because of the small size of the model. the lack of other tests, and the restrictions put on the connectivity graph of the circuit. it is extremely difficult to extrapolate the results to large sequential circuits.

Reiher and Jefferson introduce in [Reih90] a new metric called the effective utilization which is the proportion of work that is effective. Effective work is the work that is not rolled back. Based on this metric. their load balancing algorithm moves logical processes from processors which are doing a lot of effective work to other processors which are doing little effective work. The performance results presented in the paper were obtained from running two typical benchmark simulations. One was a battlefield simulation, from which they obtained an improvement of $25 \%$ of the total simulation time by using their load balancing algorithm. The other model was a simulation of two-dimensional frictionless pucks moving and colliding on a table. Since the number of pucks used was quite high relatively to the number of processors, the simulation was naturally balanced and very little improvement was observed.

Burdorf and Marti [Burd93] present a dynamic load balancing algorithm which executes on their Lisp-based Time Warp system running on a network of workstations. Their approach was motivated by the fact that users may load the workstations while a simulation was taking place. hence the need to move objects around to give the users a higher priority on computing resources. They chose the simulation time (Local Virtual Time) as a metric based on the assumption that rollbacks are extremely costly since they undo work which must be redone afterwards. Therefore. the main purpose of their algorithm is to reduce the variance between the objects simulation times. By moving onto the same processor objects which are far ahead in time and objects that are far behind, the authors believe that objects will synchronize with each other and fewer rollbacks will occur, hence speeding up the simulation. In their performance results. they find 
a five to ten fold decrease in the execution time of simulation which does not use dynamic load balancing.

Glazer [Glaz93] [Glaz93b] presents a dynamic load balancing strategy based on time slices. A time slice is a metric proportional to the ratio of the amount of computation time required by a process over its simulation advance. Once the time slice lengths are derived, processes are allocated to processors in an attempt to equalize the load on each processor. Three simulation models were constructed to represent different classes of simulation: a pipeline model, a hierarchical network model and a distributed network model. These models were ran on PARALLEX, a simulated multi-processor environment. Experimental results show that speedups ranging from $12 \%$ for the pipeline model up to $49 \%$ for the distributed network model were observed. Rollbacks were also decreased during the load balancing process, up to $50 \%$.

In [Sch195], the authors present a method for dynamic load-balancing for a simulator whose logical processes are grouped into clusters and which runs on a network of workstations. They introduce Virtual Time Progress (VTP), which reflects how fast a simulation process advances in virtual time. Virtual Time Progress is defined to be the number of simulated time units the process advances per physical time unit. Load imbalance is defined in term of a difference between the VTPs of the processors. By moving one or more clusters during the execution of the

simulation. the load is balanced by trying to make all of the VTPs approximately equal. Their results are quite encouraging since on a circuit of about 10,000 gates, they obtain a simulation runtime $20 \%$ smaller than the time needed for the same simulation without load balancing. Nevertheless, only two workstations were used for the simulation and only one circuit was tested. so it is difficult to draw any general conclusion from these performance results.

\subsection{Discussion}

Most of the existing load balancing strategies we have described above base their decision to invoke the load balancing algorithm on the progress which virtual time makes in real time (or also called GVT advance). The main objective of such an approach is to move fast and slow processes onto the same processors. This way, fast elements are then forced to slow down because they are given a lower scheduling priority (assuming that a minimum timestamp first scheduling 
policy is used). This technique assumes that rollbacks are very expensive and hopes that by forcing processes to synchronize with each other, rollbacks will be substantially decreased and the simulation will become automatically well balanced. However, this approach makes two important assumptions: uniform service times and continuous virtual time progress.

Virtual time progress based load balancing techniques do not take into consideration the fact that processes could have different service times, even though these processes perform the same amount of work for each model evaluation. A variable-delay logic simulator is an example in which processes have different service times.

Furthermore, a dynamic load balancing algorithm need to be able to evaluate whether a process move has actually improved or deteriorated the overall performance of the system so as to be able to decide of the next move. To assume that a greater virtual time progress implies automatically a better performing svistem could be a wrong premise. Figure 5.1 shows two event dependency graphs. Both graphs depict a simulation in which exactly the same number of events occurred but in which events have different dependence relations. The graph on the left hand side shows a series of events where $e_{1} \rightarrow e_{1+1}$. Clearly, $l T P=\delta_{s}$, where VTP is the virtual time progress and $\delta_{s}$ is the service time of each LP (we assume a uniform service time). The event dependency graph on the right hand side of figure 5.1 is slightly different and exhibits more parallelism. In this case $I T P=3 \cdot \delta_{*} / \overline{5}$. An advance of $3 \cdot \delta_{s}$ simulated time units is observed and 5 physical time units are required to process the 5 events. Even though both simulations contain the same number of events to be processed. their respective virtual time progress is different. Since nothing prevents the same simulation model from generating both event dependency graphs at different stages of the simulation. a load balancing algorithm which bases its decision process upon the value of the virtual time progress may interpret a decrease of the VTP value as a deterioration of the performance of the system whereas it has not actually changed. Similarly, digital logic simulation makes use of a series of input test vectors to stimulate the circuit. Each of these vectors may trigger different types of model evaluations - some of them can be very compute intensive while others may generate few events. In any case, the virtual time difference between these vectors is generally the same and has no direct relationship with the amount of work the system has to perform to evaluate the vectors. The virtual time progress can therefore vary 

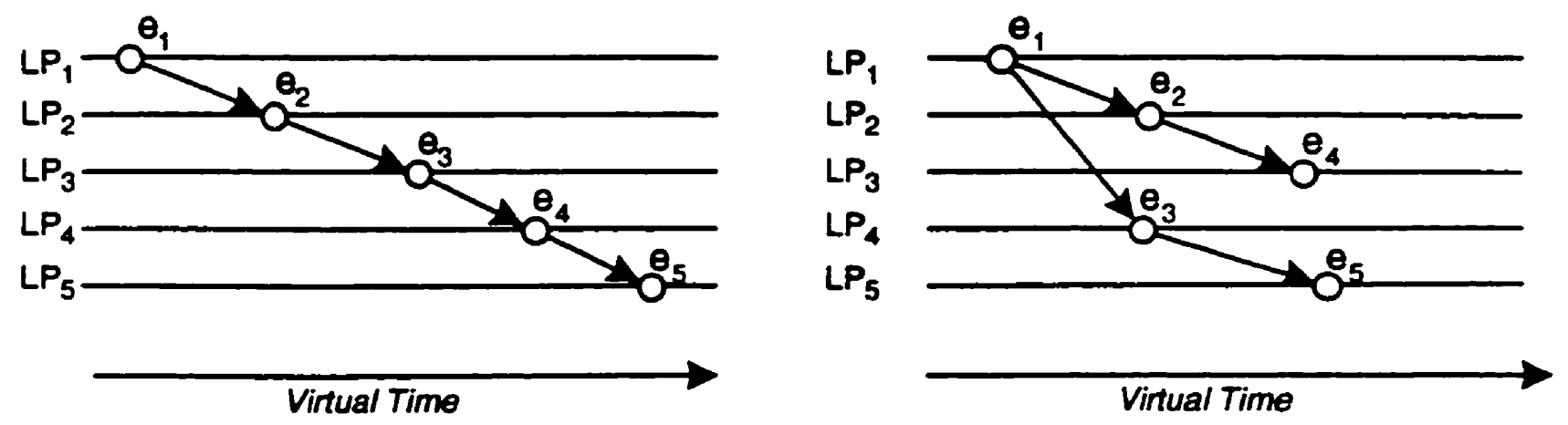

Figure 5.1: Same number of events, different virtual time progress

between input test vectors even though the performance of the system stays the same.

Because of the inadequacy of virtual time progress to measure the performance of the systems and its components, we have decided to emphasize the role of the actual process load in our dynamic load balancing algorithm and we have not used any virtual time metric. In logic simulation. the workload is not uniformly distributed among the processors and we feel that moving heavy loaded clusters onto idle processors will improve the system efficiency even at the cost of increased rollbacks.

\subsection{The Algorithm}

In this section, we describe our dynamic load balancing algorithm in detail.

\subsubsection{Workload distribution}

Due to the fine computational granularity of logic simulation, we need a metric to measure the load that is easy to compute and does not create too much overhead. We define the load of a cluster to be the number of events which were processed by its constituent logical processes since the last load balance in the simulation. This includes the rolled back events as well as the stragglers. Each processor also computes its load, which is the sum of the loads of all the clusters hosted by that processor. The load balance is improved by moving clusters from overloaded to underloaded processors. Given the load information of the clusters and the processors, our algorithm iteratively chooses the most loaded and the least loaded processors (respectively $P_{\text {heavy }}$ 
and $\left.P_{\text {light }}\right)$. The load difference $\delta$ Load of both processors is then calculated. $\delta$ Load $/ 2$ represents the load that must be transferred from $P_{\text {heavy }}$ to $P_{\text {light }}$ so that both are likely to have the same workload once the transfer has been performed. Since we want to move as few clusters as possible, we will choose the cluster whose load is the closest to $\delta$ Load/2, and assign it to $P_{\text {light }}$. The load of $P_{\text {heavy }}$ and $P_{\text {light }}$ are then updated and the same procedure is executed iteratively.

In the current implementation of Clustered Time Warp, a processor called the pilot is dedicated to collecting statistics and other types of information from the processors involved in the simulation. In order to simplify the implementation, we assigned the load balancing task to this processor. Processors periodically send their load information to the pilot by piggybacking it on the GVT token. When the pilot has decided which processor pair will be involved in the next move, the load of each cluster located in the heaviest processor is sent to the pilot which then decides which cluster to move to the lightest processor.

\subsubsection{Inter-Processor communication}

Delays created by inter-processor communications may play an important role in determining the execution time of a parallel simulation. Consequently, we extend our algorithm to incorporate the inter-processor communications. Instead of directly picking up the most heavily loaded cluster $C_{\text {heavy }}$ in processor $P_{\text {heavy }}$, we will consider all the clusters whose load is close to that of $C_{\text {heavy }}$. We say that two clusters have approximately the same load when their difference is less than a certain tolerance. In our implementation, a tolerance of $10 \%$ was used. Then for each of these clusters. we evaluate the change that would occur in inter-processor communications if it is moved to any of the lightly loaded processors. The move that minimizes communication is then chosen.

Moving a cluster $C_{k}$ from processor $P_{i}$ to processor $P_{j}$ is likely to alter the amount of communication between these two processors. It may worsen the situation since other clusters in $P_{i}$ which are communicating with $C_{k}$ will have to send events over the network. On the other hand, the situation is also improved since clusters in $P_{j}$ which were communicating with $C_{k}$ will not need to send messages over the network anymore. Therefore the overall change in communication load is: 


$$
\delta I P C\left(C_{k}, P_{i}, P_{j}\right)=\sum_{v C_{n} \in P_{j}} I C C\left(C_{k}, C_{n}\right)-\sum_{v C_{m} \in P_{i}} I C C\left(C_{k}, C_{m}\right)
$$

where $\operatorname{ICC}\left(C_{a}, C_{b}\right)$ is the number of messages exchanged between clusters $C_{a}$ and $C_{b}$. It

is referred to as Inter-Cluster Communication. The number of messages is calculated over a certain period of time which must be long enough so that the measure can be considered as reliable. In the case of logic simulation, this period of time must include the processing of at least one input vector so that all parts of the circuit have a chance to be activated. Since the load balancing algorithm is not activated until the whole system becomes stable. several input vectors are already processed at this stage.

\subsubsection{Pseudocode}

Figure 5.2 shows the pseudocode for the load balancing algorithm whose objective is to choose the identity of the next cluster to move and its new location while maximizing the load balance and trying to minimize inter-processor communication at the same time. 


\section{Inputs}

$\Pi$ is the set of all processors.

\section{Outputs}

$C_{\text {move }}$ is the cluster to move.

$P_{\text {dest }}$ is the destination processor of $C_{\text {move }}$.

\section{begin}

(1) $P_{\text {dest }} \leftarrow \emptyset$

(2) $C_{\text {move }} \leftarrow \emptyset$

(3) select $P_{\text {light }} \in \Pi \mid \operatorname{Load}\left(P_{\text {light }}\right)=\operatorname{Min}\left(\operatorname{Load}\left(P_{i}\right)\right) \forall P_{i} \in \Pi$

(4) select $P_{\text {heavy }} \in \Pi \mid \operatorname{Load}\left(P_{\text {heary }}\right)=\operatorname{Max}\left(\operatorname{Load}\left(P_{i}\right)\right) \forall P_{i} \in \Pi$

(5) $\delta$ Load $\leftarrow \operatorname{Load}\left(P_{\text {heavy }}\right)-\operatorname{Load}\left(P_{\text {light }}\right)$

(6) let $\Gamma_{c} \mid \forall C_{i} \in \Gamma_{c} \Rightarrow \operatorname{Load}\left(C_{i}\right)<\delta \operatorname{Load} / 2$ and $C_{i} \in P_{\text {heavy }}$

(i) select $C_{\text {heavy }} \in \Gamma_{c} \mid \operatorname{Load}\left(C_{\text {heary }}\right)=\operatorname{Max}\left(\operatorname{Load}\left(C_{i}\right)\right) \forall C_{i} \in \Gamma_{c}$

(8) for each $C_{i} \in \Gamma_{c} \mid \operatorname{Load}\left(C_{1}\right) \approx \operatorname{Load}\left(C_{\text {heavy }}\right)$

(9) for each $P_{j} \in \Pi \mid \operatorname{Load}\left(P_{\text {heavy }}\right)-\operatorname{Load}\left(P_{j}\right)>2 . \operatorname{Load}\left(C_{i}\right)$

(10) if $\delta I P C\left(C_{1}, P_{\text {heary }} . P_{\jmath}\right)<\delta I P C\left(C_{\text {move }}, P_{\text {heavy }}, P_{\text {dest }}\right)$

$$
\begin{aligned}
& P_{\text {dest }} \leftarrow P_{J} \\
& C_{\text {move }} \leftarrow C_{t}
\end{aligned}
$$

endif

endfor

endfor

end.

Figure 5.2: Pseudocode of the load balancing algorithm 


\subsubsection{Triggering the load-balancing algorithm}

Once a move has been decided upon, the loads of the two processors involved in the transfer are reevaluated. Then a decision process is started to find what cluster to move and where to move it. This procedure will converge to a better mapping of clusters, but not necessarily to the optimal one. This process can be repeated until the estimated workload distribution cannot be improved upon. Nevertheless, this procedure would not be very realistic for two main reasons. First, there is no control over the number of moves. Second, even though the processors' loads are reevaluated each time a move has been decided upon, the newly evaluated loads do not necessarily reflect the actual resulting load of the processors, mainly because the loads of the other processors have changed. Therefore, an iterative method was used. At each step of the load-balancing algorithm, only a certain number of clusters will be allowed to move. Then the system waits until the following two conditions are satisfied before triggering other moves:

- The cost of moving the clusters has been amortized by the resulting speedup.

- New up-to-date measures are available.

Our load balancing triggering mechanism is based on the throughput of the simulation system. defined as the number of non rolled-back message events per unit time. The throughput does not include anti-messages. In the domain of logic simulation. we feel the throughput is a better measure of the overall speed of the simulation than the GVT advance which is more dependant on the nature of the model and its behavior. For example, a large advance in the GVT can be achieved by processing a small number of events.

Because more events get committed as the simulation progresses, fewer objects are being manipulated by the system. thus decreasing the overhead. On the other hand. if more input vectors are applied to the primary inputs of the simulated circuit. the overhead will tend to increase. Consequently, since the throughput may fluctuate over time, we use a least-squares approximation [Watk91, Corm91] to obtain the general trend from a series of discrete instant values of the throughput. In our experiments, a first-degree equation gave very satisfactory results, nevertheless. calculating the mean would not have been sufficient since the derivative of the throughput 
was not nil. The stability of the system is detected when coefficients obtained from the approximation are considered to be constant as new throughputs are calculated. In our implementation, a throughput point is calculated every 3 seconds, the least-squares approximation spans 6 points and the coefficients are considered to be constant if their value does not change more than $5 \%$.

The load balancing mechanism is triggered for the first time when the stability of the system is detected. When a move is initiated at real time $t=t_{\text {start }}$, the throughput of the system tends to decrease since processors are spending their time transferring clusters. Then. once the operation is completed, the throughput increases, reaches its original value at $t=t_{\text {even }}$ which is equivalent to the break-even time (as defined in [Schl95]). The throughput finally reaches a stable value larger than that previous to the transfer. Figure 5.3 depicts this situation.

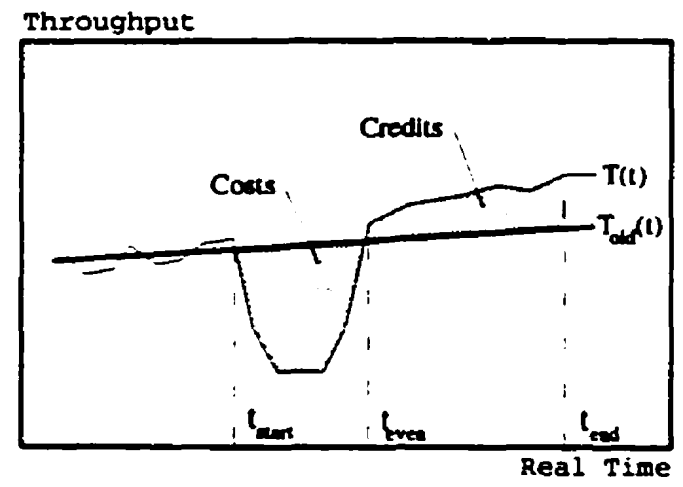

Figure 5.3: Throughput during a transfer

Triggering the load balancing mechanism each time the stability is detected might actually decrease the overall speed of the simulation, even if the final throughput is higher than the previous one. As we have seen, moving clusters around has a cost in terms of throughput which is represented by the shaded area in the interval $\left[t_{\text {start }}, t_{\text {even }}\right]$ in figure 5.3. On the other hand, the transfer has the benefit of ultimately increasing the overall throughput of the system and improving its overall performance. The shaded area in the interval [ $\left.t_{\text {even }}, t_{\text {end }}\right]$ in figure 5.3 represents that improvement. If the next load balancing mechanism is triggered before the gain in throughput is equal to the cost of the transfer, the overall speed of the simulation might actually decrease. As a consequence, before launching the next load balancing mechanism, we 
wait at least until the following condition is satisfied:

$$
\int_{t_{\text {otert }}}^{t_{\text {end }}} T(t)=\int_{t_{\text {ouert }}}^{t_{\text {end }}} T_{\alpha d d}(t)
$$

where $T_{\text {old }}(t)$ is the approximation of the throughput before the transfer and $T(t)$ is the actual throughput.

Observations have indicated that as the simulation progresses, the improvements become less significant and the period between each balancing adjustment grows longer. Once the cost of moving clusters does not improve the throughput so as to "pay back" the cost of the transfers, the load balancing mechanism is halted.

\subsection{Metrics}

To measure the effect of the load balancing mechanism, we define three metrics which depict different characteristics of the simulation system.

\subsection{1 $\beta$ : Workload distribution}

To measure the quality of the load-balance. we define $\beta$ as the ratio of the standard deviation of the processor loads to the maximum load observed. The lower that 3 is, the more equally the load is distributed.

$$
\beta=\frac{1}{\operatorname{Load}_{\max }} \sqrt{\frac{\sum_{i=0}^{n-1}\left(\operatorname{Load}\left(P_{i}\right)-\overline{\operatorname{Load}}\right)^{2}}{n-1}}
$$

where $n=|\Pi|$ and $\Pi$ is the set of all processors,

$$
\begin{aligned}
& \operatorname{Load}_{\max }=\operatorname{Max}\left(\operatorname{Load}\left(P_{i}\right)\right) \forall P_{i} \in \Pi \text { and } \\
& \overline{\operatorname{Load}}=\frac{\sum_{\mathrm{i}=0}^{n-1} \operatorname{Load}\left(P_{i}\right)}{n}
\end{aligned}
$$




\subsection{2 $\gamma$ : Inter-processor communication}

We define $\gamma$ as being the ratio of the number of events exchanged between processors $\left(\Gamma_{i p c}\right)$ to the number of events exchanged between clusters $\left(\Gamma_{\text {total }}\right)$. The lower that $\gamma$ is, the lower is the inter-processor communication.

$$
\gamma=\Gamma_{\text {ipe }} / \Gamma_{\text {total }}
$$

\subsection{3 $\rho$ : Cancelled computation}

$\rho$ is defined as the ratio of the number of events rolled back ( $\left.E_{\text {cancelled }}\right)$ to the total number of events processed ( $E_{\text {processed }}$ ). The lower that $\rho$ is, the less computation is cancelled.

$$
\rho=E_{\text {cancelled }} / E_{\text {processed }}
$$

The three metrics $\beta, \gamma$, and $\rho$ are measured over the same period of time as the throughput and only their mean calculated over the six previous points is considered.

\subsection{Implementation}

In Clustered Time Warp, each gate of a digital circuit is modelled by a Logical Process. LPs are then grouped into clusters which are in turn mapped onto processors. Several clusters may reside on the same node, but a single cluster cannot be split among different processors.

Three checkpointing algorithms were developed for CTW:

- CRCC Clustered Rollback. Clustered Checkpoint.

- LRCC Local Rollback. Clustered Checkpoint.

- LRLC Local Rollback, Local Checkpoint.

Each of these techniques offers a different memory vs. execution time trade-off [Avri95]. CRCC is the least expensive in terms of memory, and LRLC is the least expensive in terms of time. In our experiments, we make exclusive use of LRCC since it offers an intermediate choice for both of these characteristics. 
Cluster sizes in the range of 50 to 200 gates were experimented with. Since little difference was observed between these sizes, we present the results for 100 gates.

We used a string partitioning algorithm, because of its simplicity and especially because results have shown that it favors concurrency over cone partitioning [Brin91].

The dynamic load balancing algorithm was implemented on top of Clustered Time Warp and run on a BBN Butterfly GP1000 multiprocessor. The implementation of message passing in the simulation is independent of the shared memory of the Butterfly. Consequently our results will apply to distributed memory architectures (see section 4.1).

Moving a cluster from one processor to another is a 2-phase operation. First. the sending processor encodes the data structure of the cluster into a message and then sends it to the receiving processor. While the transfer is taking place, events are still sent to the original processor which stores them in a forward list. Once the transfer is over. the second phase of the transfer starts. The receiving processor sends an acknowledgment to the sending one which then sends it the forward list and broadcasts to all the other processors the new location of the cluster. Even though routing tables are updated immediately, due to variable communication delays it is still possible for a processor to receive messages for a cluster that has been moved away. In this case, the message is simply forwarded to the correct processor.

\subsubsection{Experiments}

We conducted a series of experiments in order to determine how well our dynamic load-balancing algorithm performs when compared to Time Warp. We also tried to measure the effects of load distribution. inter-processor communication and rollbacks on the overall performance of the simulation. The circuits used in our study are digital circuits selected from the ISCAS'89 benchmarks. We present the results obtained from simulations of two of the largest circuits (table 5.1) which are both representative of the results which we obtained with other circuits.

\subsubsection{Workload Distribution}

We first examined Balanced Clustered Time Warp (BCTW) in which our load balancing algorithm tries to improve the workload distribution without considering inter-processor communications 


\begin{tabular}{|c|c|c|c|c|c|c|}
\hline name & \# inputs & \# outputs & \# flip-flops & total & \multicolumn{2}{|c|}{ Asynchronous Parallelism } \\
\cline { 5 - 6 } & & & & & Average & Relative \\
\hline s38417 & 28 & 106 & 1,636 & 23,949 & 650 & $2.71 \%$ \\
\hline s38584 & 12 & 278 & 1,452 & 20,995 & 1.107 & $5.27 \%$ \\
\hline
\end{tabular}

Table 5.1: Circuits s38417 and s38584

and rollbacks. To this end, a series of simulations were run on 20 processors, with pure Time Warp (TW), with Clustered Time Warp (CTW), and BCTW. The execution time of each simulation was between 250 and 300 seconds when the pure Time Warp approach was used. Figures 5.4 and $5 . \overline{5}$ show the progress of the Global Virtual Time versus the Real Time for circuits s3841 and s38584 respectively, using TW. CTW and BCTW.

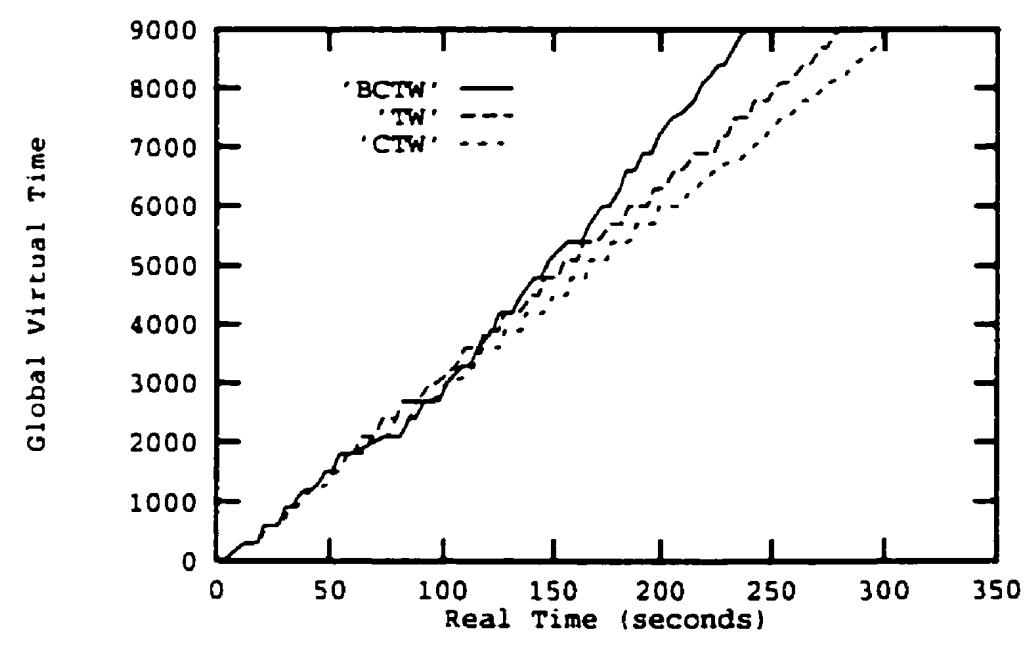

Figure 5.4: GVT Advance for circuit s38417

For both circuits. the total simulation time has been substantially decreased when load balancing was used. For circuit s3841 $\%$, the Balanced Clustered Time Warp algorithm is about $15 \%$ faster than pure Time Warp and $25 \%$ faster than the unbalanced version of CTW. As for circuit s38584, BCTW was about $40 \%$ faster than Time Warp and $50 \%$ faster than CTW.

The reason why load balancing had a more pronounced effect on circuit s38584 may be in part found in the greater parallelism available in that circuit. Table 5.1 indicates that the average 


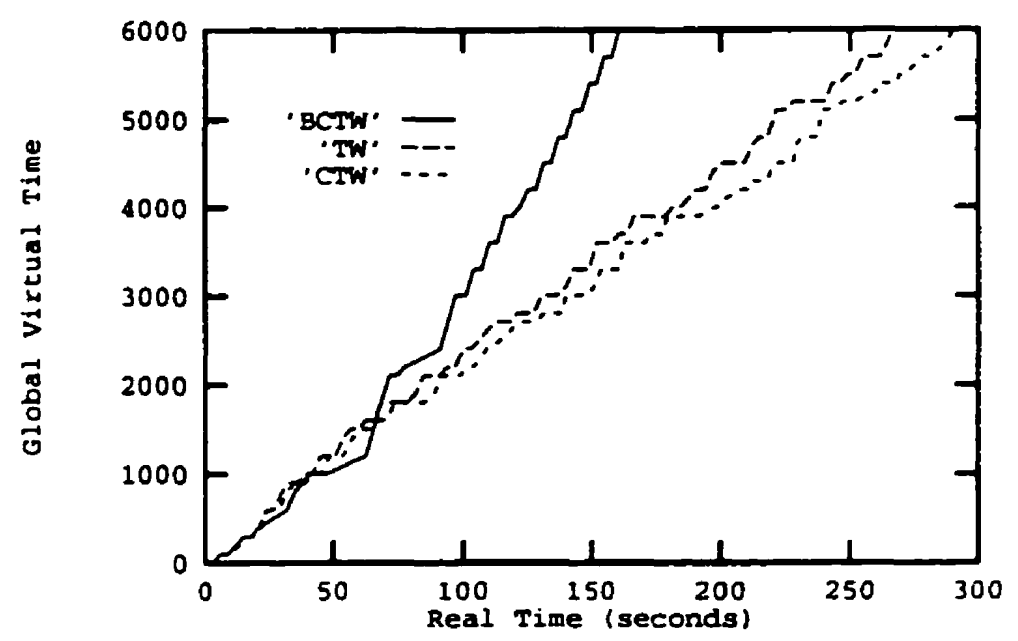

Figure 5.5: GVT Advance for circuit s38584

parallelism in circuit s38584 is more than twice as high as in circuit s38417. Furthermore, the workload distribution in both circuits is fairly poor (see chapter 2), therefore it is likely that most of the work will be concentrated on a subset of the processors while the others have very little work to do. As a consequence, moving clusters out of the overloaded processors and assigning them to underloaded processors tend to speed up the whole simulation process for circuit 538584 more effectively in which more concurrency is available.

Both circuits were partitioned into 200 clusters of equal size, which gives enough flexibility for the balancing algorithm to transfer load between processors. If the circuits are partitioned into too many clusters. the system would have to handle more information, and the advantage of Clustered Time Warp would be lost. On the other hand, if the number of clusters is too small. the load balancing algorithm would have a very limited choice of clusters to move. consequently. the improvements are limited. In order not to overload the system. the maximum number of clusters to be moved during each transfer was set to 15 .

For the simulation of circuit $s 3841 \bar{i}$, the first transfer of clusters was triggered by the algorithm at real time $t_{r}=60 \mathrm{~s}$, the second one at $t_{r}=102 \mathrm{~s}$, and the last one at $t_{r}=152 \mathrm{~s}$. As for circuit s38584, the load balancing algorithm also transferred clusters three times at $t_{r}=21 s, t_{r}=51 \mathrm{~s}$ and at $t_{r}=81 \mathrm{~s}$. The effect on the GVT advance of the second transfer for circuit s38584 is actually very visible in figure 5.5. For BCTW, the GVT advance becomes suddenly smaller than 
that of TW and CTW until $t_{r}=i 0 s$ approximately, at which time processors previously involved in the transfer can resume their simulation work.

Figure 5.6 and 5.7 shows the variation of the three metrics 3 (load imbalance). $\gamma$ (interprocessor communication) and $\rho$ (rollbacks) during the simulation run of circuits s3841 $i$ and s38584 respectively using the Clustered Time Warp engine along with the dynamic load balancing mechanism.

The load imbalance $(\beta)$ of circuit $s 38417$ (figure 5.6) starts actually with a very high value

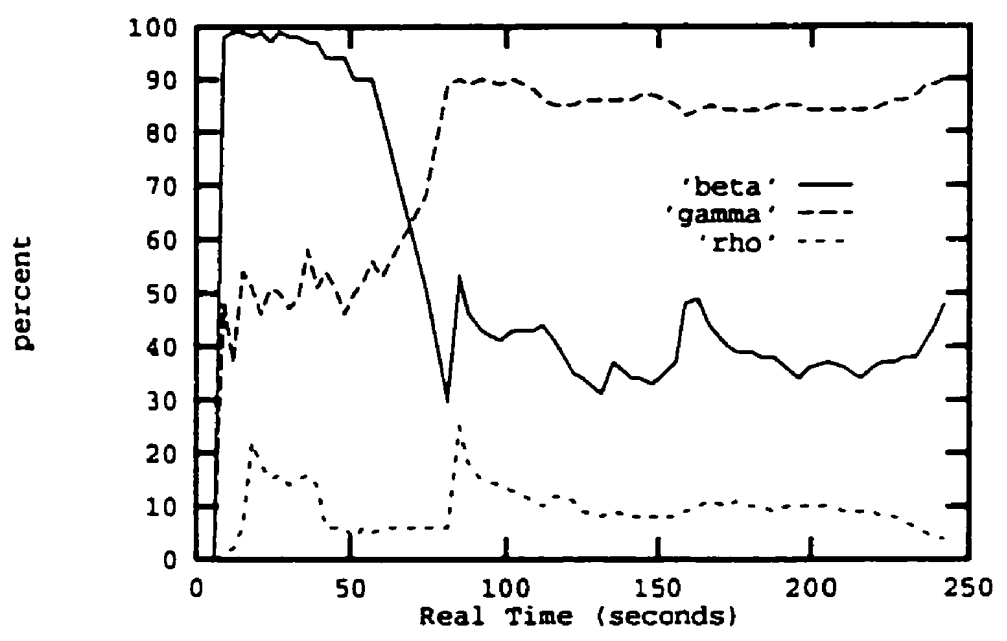

Figure 5.6: $3, \gamma$ and $\rho$ for circuit s38417

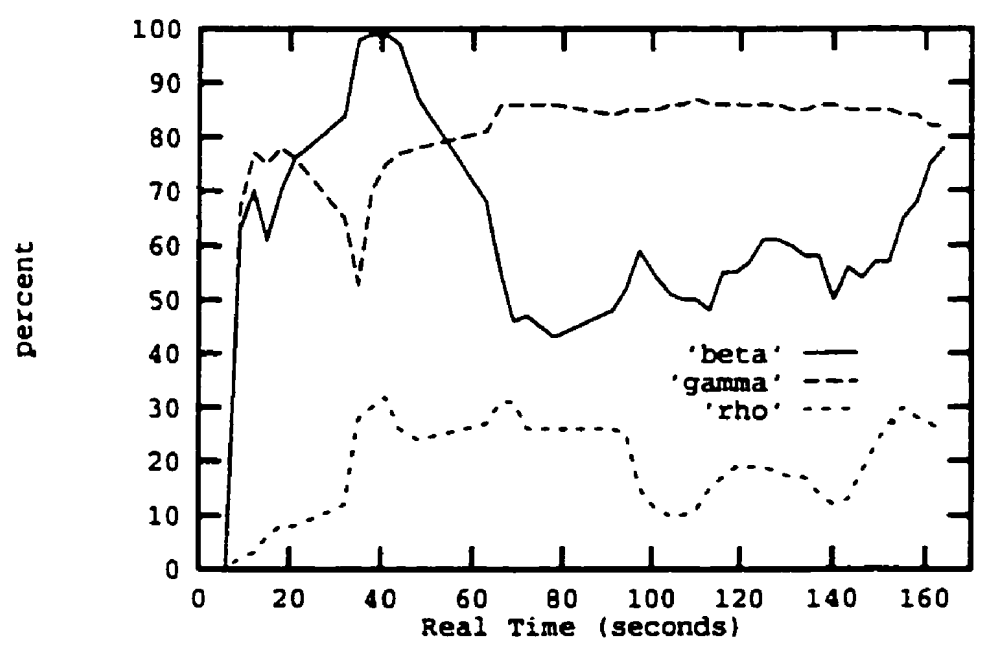

Figure 5.7: $\beta, \gamma$ and $\rho$ for circuit s38584 
(hence a very bad load balance). The first transfer of clusters was triggered about 50 seconds after the beginning of the simulation. For a period of about 10 to 20 seconds, overloaded processors are busy sending the states of the clusters and logical processes which have been assigned to underloaded processors. Once clusters of LPs have been transferred, 3 suddenly decreases from about $98 \%$ down to less than $40 \%$, indicating that the load is more distributed among the processors. However, the improvement observed for the load balance caused the opposite effect for inter-processor communication $(\gamma)$ whose value is doubled. This increase was expected since loaded clusters have been more evenly distributed over the processors. Nevertheless. no substantial change can be observed regarding the rollbacks $(\rho)$.

In figure $5 . \tau$. we show the variation of $\beta, \gamma$ and $\rho$ during the simulation of circuit $s 38584$. Shortly after the first transfer which has been initiated 21 seconds after the beginning of the simulation. we observe a sudden decrease of inter-processor communication which is caused by the fact that overloaded processors (which are most likely to send messages) are busy sending clusters to underloaded processors. As a direct consequence, the load imbalance $(3)$ increases. When the transfer is completed, similarly to circuit s38417, 3 starts to decrease and $\gamma$ increases. We can observe that about $86 \%$ of the messages exchanged between clusters are sent over the communication network. instead of $7 \pi \%$ previous to transfer. Unlike circuit s3841 $\boldsymbol{T}$. the number of rollbacks observed during the simulation of circuit s38584 increases dramatically from about $10 \%$ before the transfer to about 20 to $30 \%$ after the transfer.

Nevertheless. despite the costs created by extra rollbacks and inter-processor communications, the overall throughput of the system increases. Even though the effect was less pronounced. we observed the same phenomenon for circuit s38417.

Figures 5.8 and 5.9 shows the impact of the load balancing algorithm on the throughput of the system during the simulation of circuits 338417 and s38584 respectively. In the case of circuit s38417 (figure 5.8), the first transfer which was triggered about 50 seconds after the beginning of the simulation caused the throughput to decrease considerably. Even though its value reaches that of Time Warp soon after, any substantial improvement can be observed only after the third transfer. As for circuit s38584 (figure 5.9), we observe that soon after the first transfer of clusters 


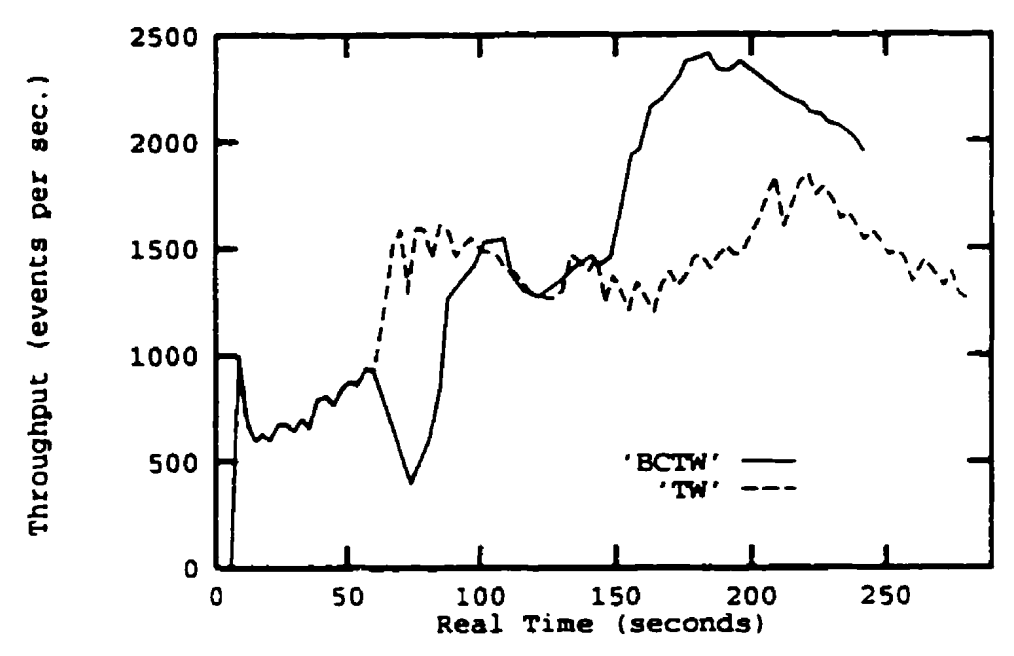

Figure 5.8: Throughput for circuit s38417

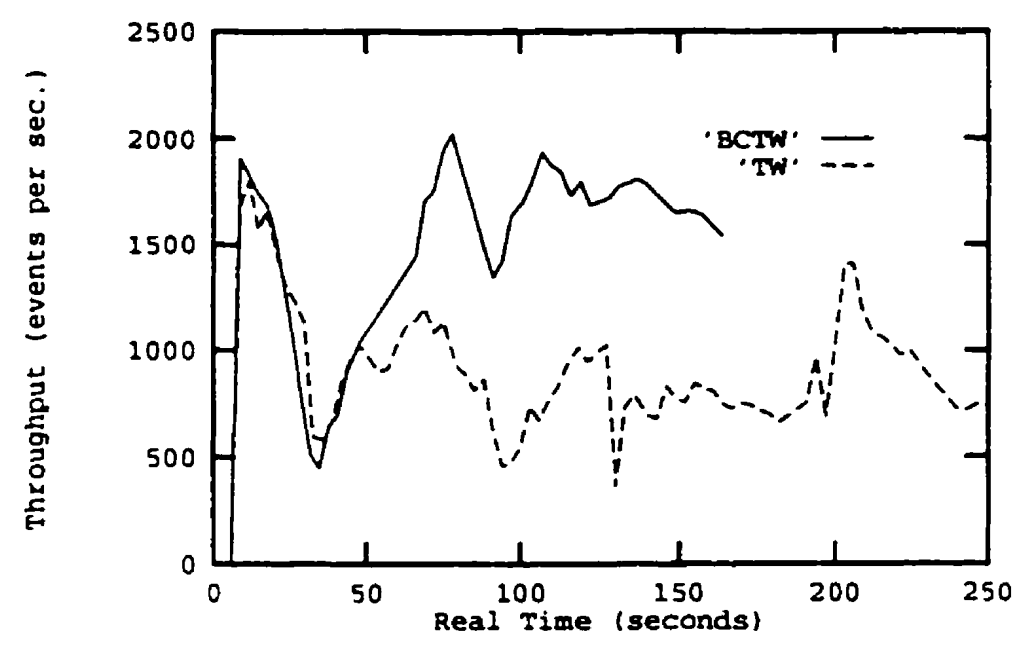

Figure 5.9: Throughput for circuit s38584

is initiated (at $t_{r}=20 \mathrm{~s}$ ), the throughput becomes smaller than that of Time Warp, then once the transfers are over, it becomes quickly twice as large as that of Time Warp.

We also observe that the throughput for circuit s38584 is substantially smaller than that for circuit $s 38417$, even though the available parallelism in the circuit is as twice as high. Because of the load imbalance at the beginning of the simulation of circuit s38584, heavily loaded processors have actually a much higher synchronization overhead than the processors during the simulation of circuit $s 38417$. For example, the largest size observed for the event heap in the simulation was 


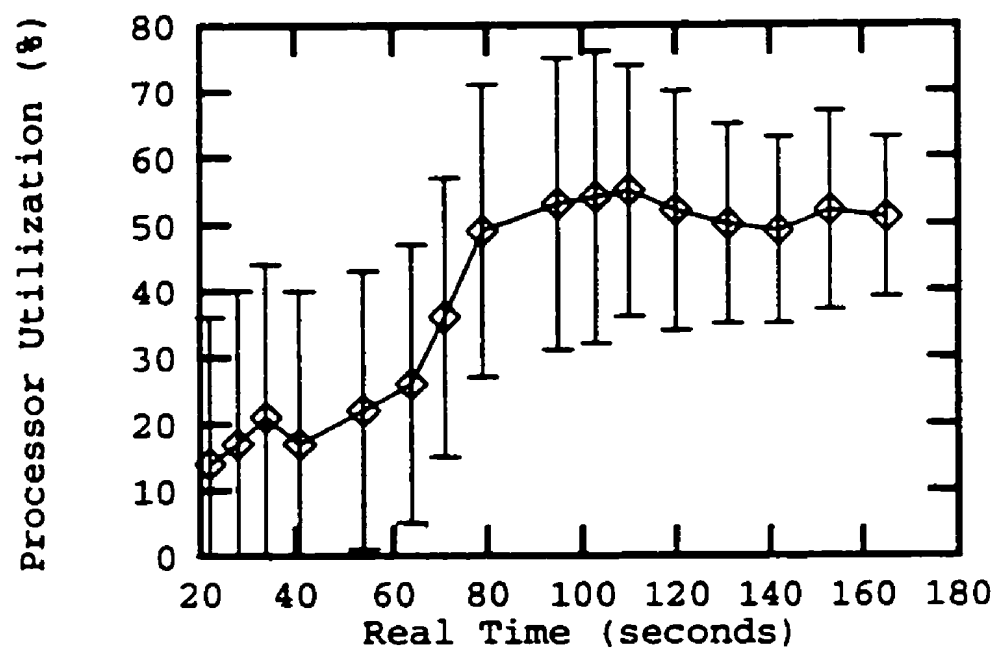

Figure 5.10: Mean and S.D. of the processor utilization for circuit s38584

3.587 events for circuit s38584, while the one observed for circuit s3841 7 was only 1.557 events. As a consequence, more compute resources are wasted on housekeeping for circuit s38584.

For each processor, we also measured the process utilization which is defined as the percentage of time spent on computation (including synchronization overhead) during a certain period of time. At different points in the simulation. we calculated the mean and the standard deviation of the processor utilizations. The results are given in figure 5.10 for circuit s38584. They show that as the load balancing mechanism transfers clusters from overloaded to underloaded processors. the utilization rises from $15 \%$ up to $50 \%$, and the standard deviation decreases by about $40 \%$. This proves that our load balancing algorithm succeeded in distributing the computation load over the processors. As a result, the system is able to extract more parallelism from the simulation model and as a consequence the overall performance is substantially improved.

\subsubsection{Inter-processor Communications}

Another series of simulations were run in which the load balancing algorithm also attempts to minimize inter-processor communication while still trying to improve the load distribution (Balanced Clustered Time Warp with IPC or BCTW+IPC). Figures 5.11 and 5.12 show for both circuits s38417 and s38584 respectively, the variation of the metric $\gamma$ (inter-processor commu- 


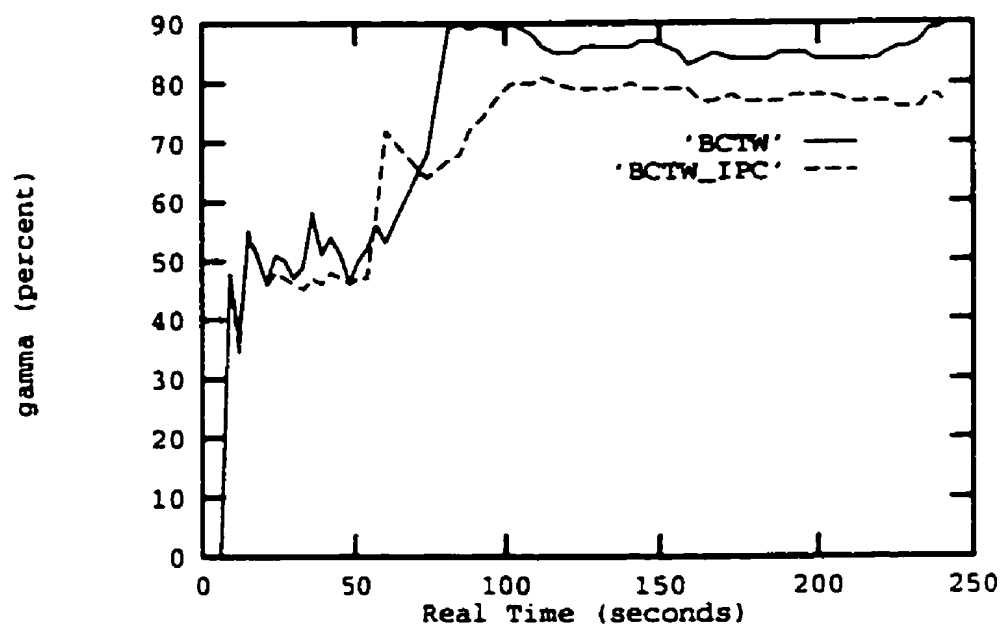

Figure 5.11: Effect on $\gamma$ for circuit s38417 if IPC is minimized

nication) over real time. In each case, we observe that the algorithm succeeded in decreasing inter-processor communications by about $5-8 \%$. This slight improvement also translates into a decrease of the amount of computation cancelled.

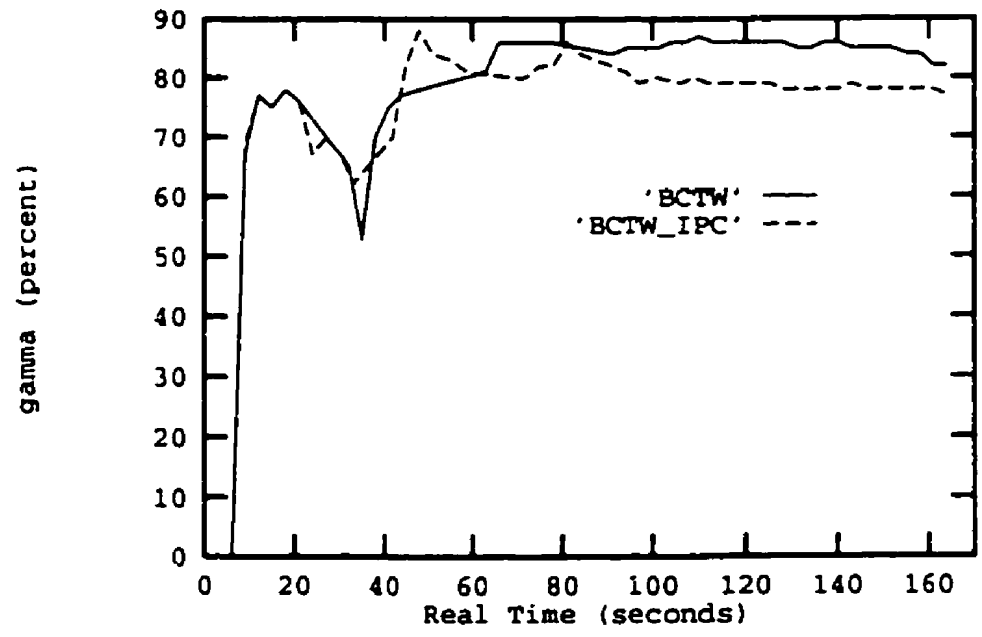

Figure 5.12: Effect on $\gamma$ for circuit s38584 if IPC is minimized

Figures 5.13 and 5.14 show the variation of $\rho$ over real time for both circuits s38417 and s38584 respectively where $\rho$ represents the proportion of rolled back events in the simulation. 


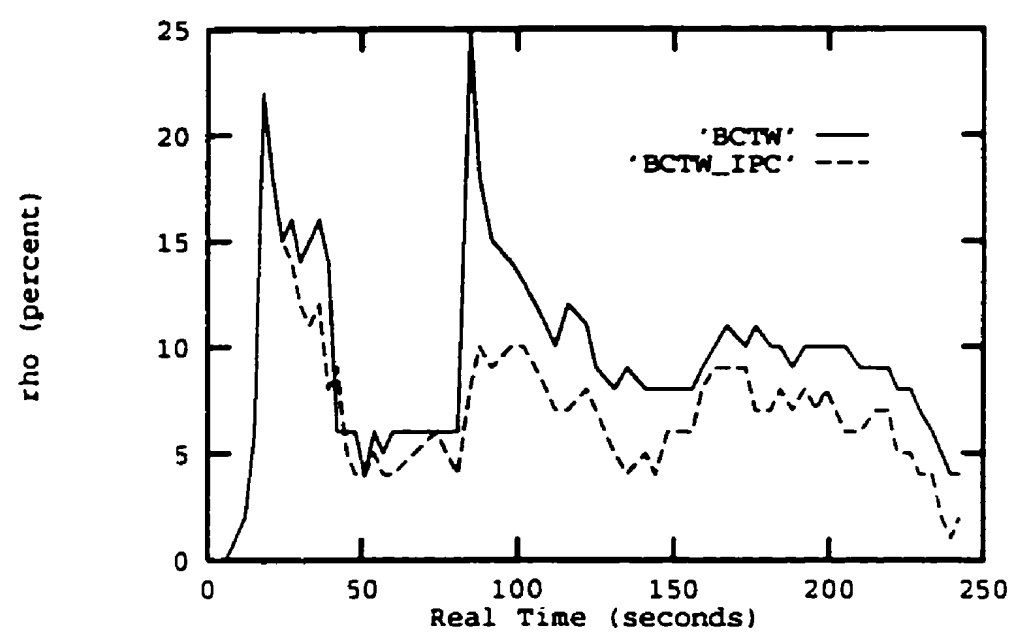

Figure 5.13: Effect on $\rho$ for circuit s38417 if IPC is minimized

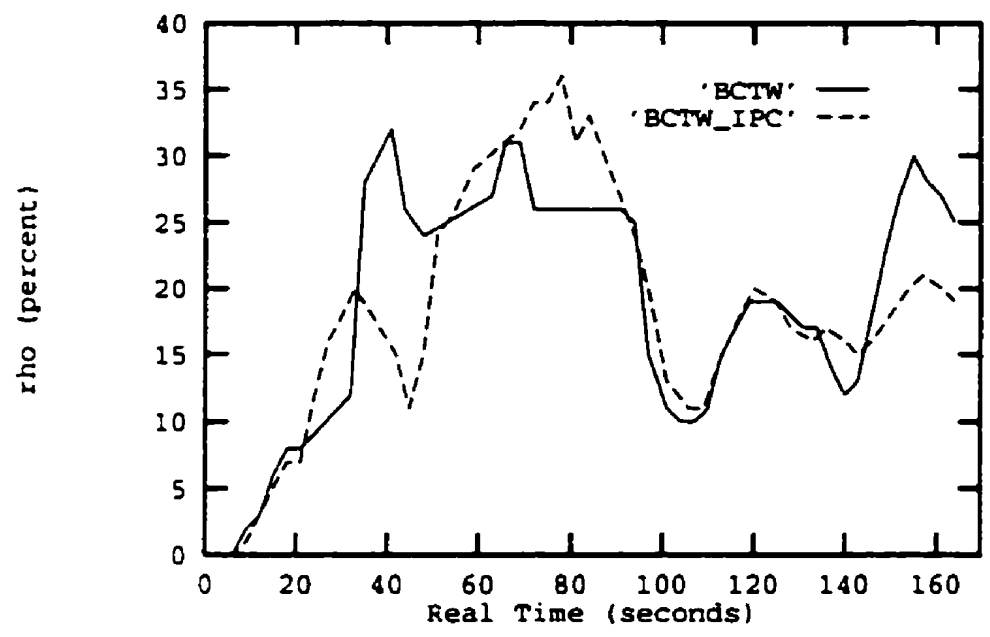

Figure 5.14: Effect on $\rho$ for circuit $\$ 38584$ if IPC is minimized

Each figure depicts the results for both Balanced Clustered Time Warp and BCTW taking inter-processor communication into consideration. In both cases, the total amount of computation cancelled by rollbacks has been substantially reduced when the load balancing algorithm tried to minimize inter-processor communication, even though at some points for circuit s38584, the proportion of rollbacks increased. This shows the strong correlation between IPC and rollbacks. 


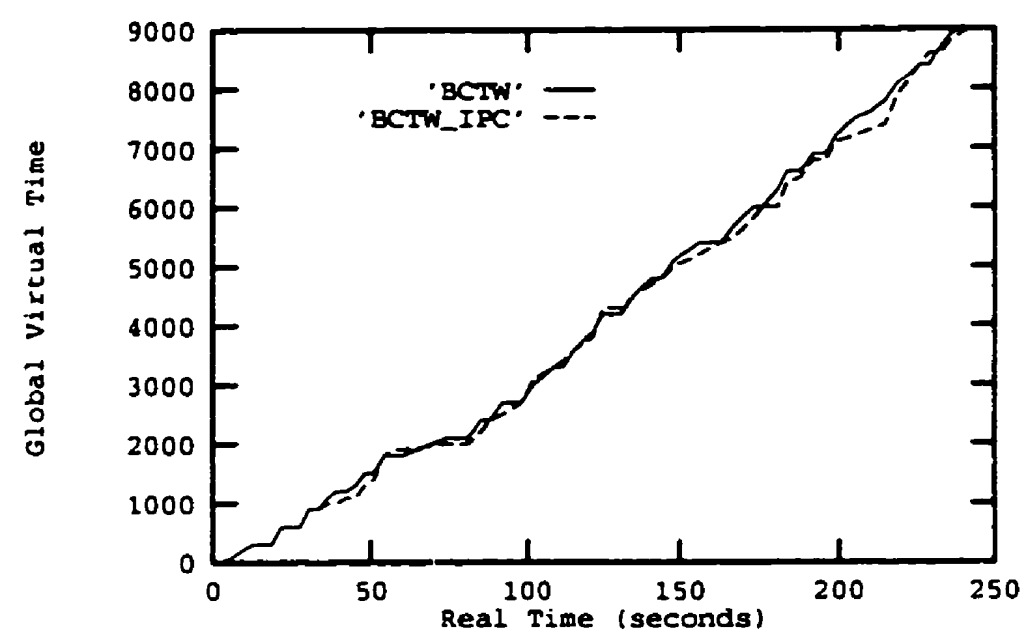

Figure 5.15: Effect on the GVT Advance for circuit $\$ 38417$ if IPC is minimized

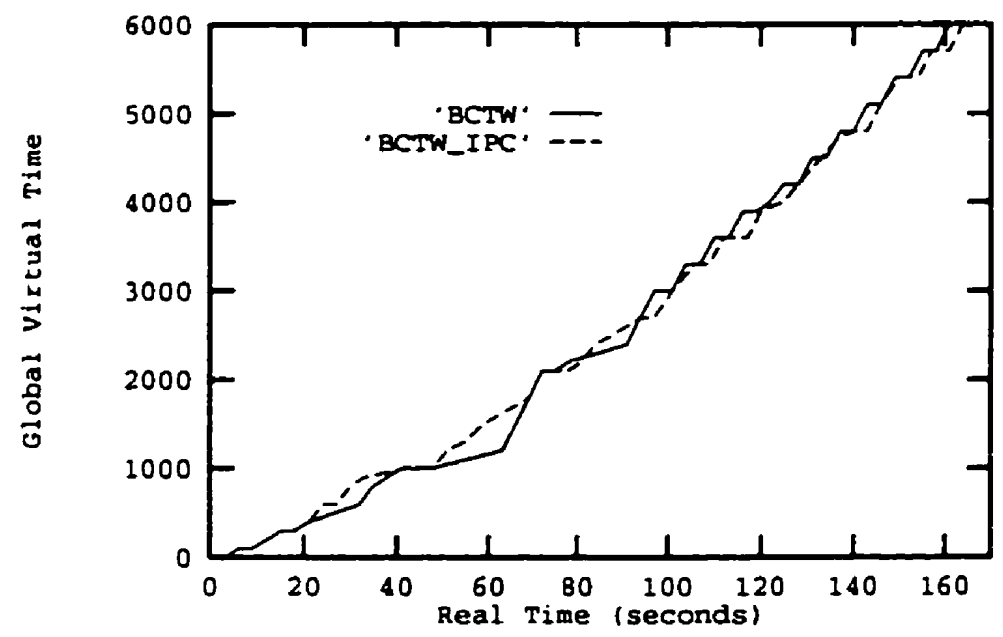

Figure 5.16: Effect on the GVT Advance for s38584 if IPC is minimized

Nevertheless, the reduction of inter-processor communications and rollbacks did not improve the overall performance of the simulation. Figure 5.15 for circuit $s 38417$, and figure 5.16 for circuit s38584 show the advance of the Global Virtual Time versus real time for Clustered Time Warp with load balancing (BCTW), and Clustered Time Warp with load balancing considering interprocessor communication (BCTW+IPC). Both algorithms perform similarly and no substantial increase in the speed of the simulation is observed when inter-processor communication is reduced. 


\subsubsection{Memory requirements}

Figures 5.17 and 5.18 show the peak memory usage of circuits s3841 7 and s38584 respectively. Each graph shows the results obtained from using Time Warp (TW), Clustered Time Warp (CTW) and Balanced Clustered Time Warp (BCTW). For both circuits, we observe that by dynamically balancing the load among the processors, the maximum memory requirements are substantially reduced. For circuit s38417, the peak memory usage of Balanced Clustered Time

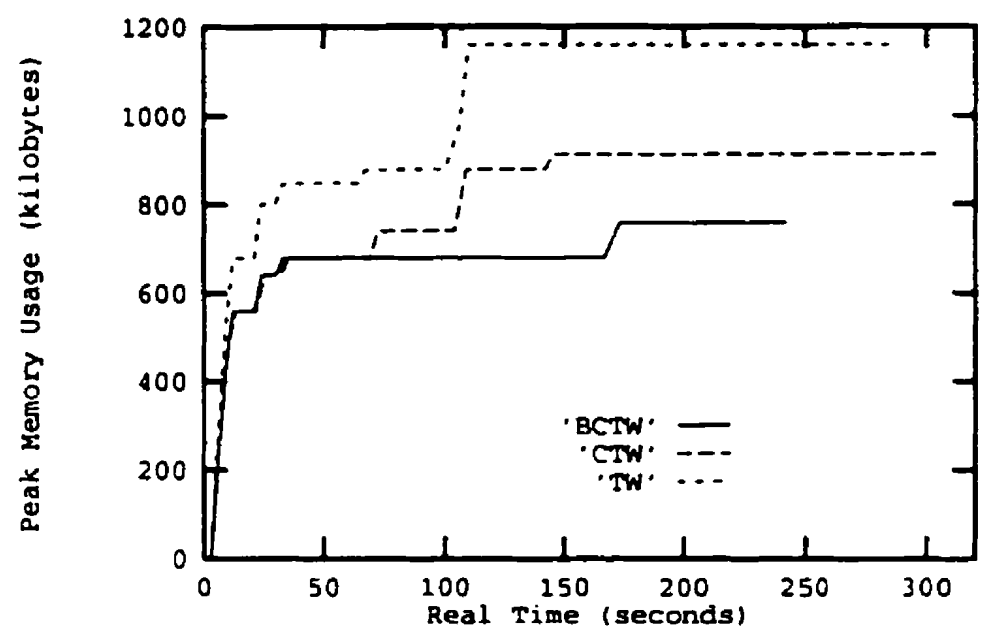

Figure 5.17: Peak Memory usage for circuit s38417

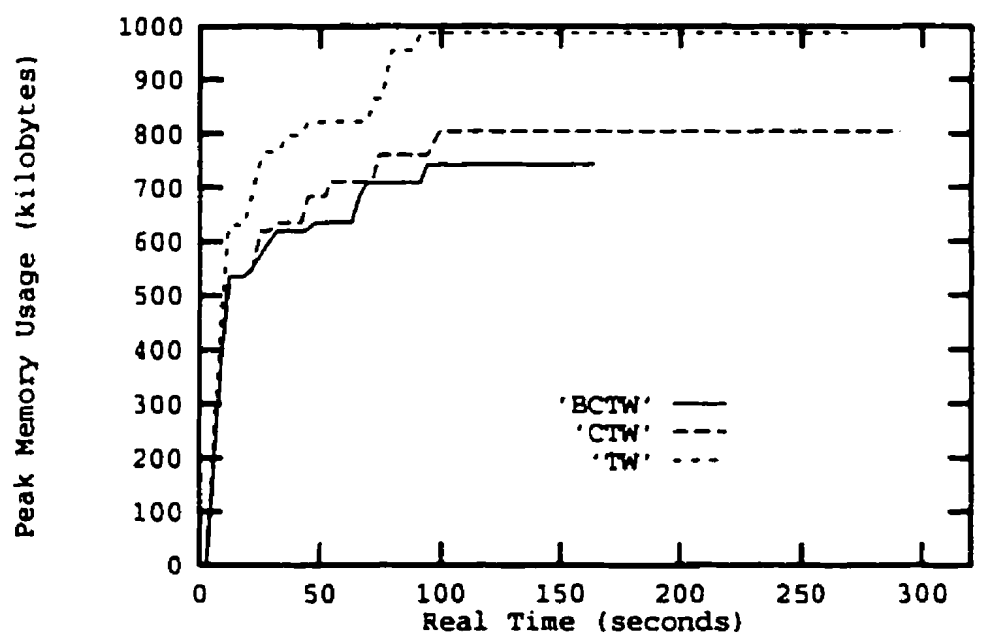

Figure 5.18: Peak Memory usage for circuit s38584 
Warp is $16 \%$ smaller than that of Clustered Time Warp and $34 \%$ smaller than Time Warp. Similarly, for circuit s38584, the peak memory usage of Clustered Time Warp is reduced by $8 \%$ when dynamic load balancing is used, and by $25 \%$ when compared to Time Warp.

The reason why the memory usage was not reduced for circuit s38584 as much as for circuit s3841 7 is due to the fact that locality of the workload is stronger for s38584 than for circuit s3841i. A stronger locality results in a more unbalanced simulation, and some processors end up having more processes having a high activity. As a consequence the memory needed to store their states and events is likely to be larger too.

\subsubsection{Throughput vs. Number of Processors}

Figure 5.19 depicts the improvement of the throughput obtained from using the load balancing algorithm with different numbers of processors. We observe that up to 20 processors, the load balancing algorithm is more effective in accelerating the simulation. When a small number of processors are used, the load on the processors is much higher, as a consequence. little room is left to improve the load balancing.

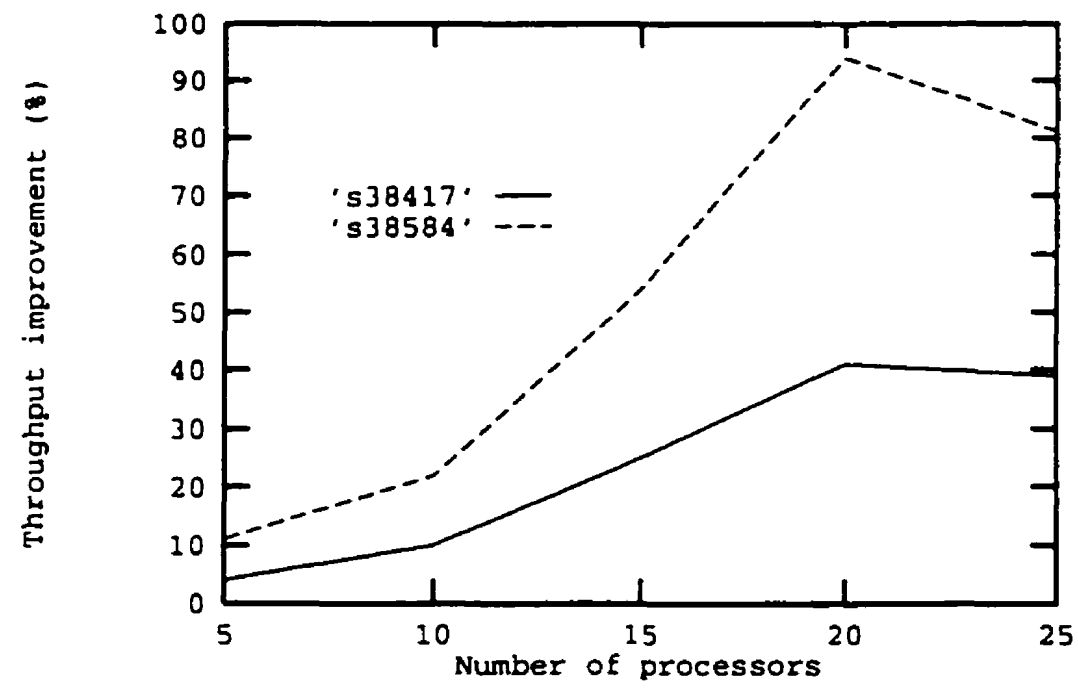

Figure 5.19: Improvement of the Throughput 
However. we also observe that for both circuits, the throughput improvement starts to decrease from 20 processors onward. This phenomenon is explained by the fact that the overhead incurred by the synchronization algorithm itself becomes larger. The dissemination of processes among more processors tends to extract more parallelism, but on the other hand. the synchronization overhead (housekeeping, inter-processor communications, rollbacks. etc.) has a more pronounced effect. Relatively to the number of processes per processor, the work needed to preserve causality in the system becomes less negligible, especially if the granularity of the computation is small (as in our case).

\subsection{Summary}

We have described in this chapter a dynamic load-balancing algorithm for Clustered Time Warp. The advantage of the clustering approach to load balancing is that instead of having to move individual LPs from one processor to another, clusters of LPs can be moved.

We have also described a triggering technique based on the throughput of the simulation system. Throughput is the number of non rolled-back message events per unit time.

We have shown that a substantial acceleration of the simulation speed can be obtained. Two circuits of more than 20,000 gates were tested and improvements of 40 and $100 \%$ were obtained for the throughput. However, the reduction of the simulation time is limited by the intrinsic parallelism of the model. A load balancing technique is likely to perform better if the asynchronous parallelism of a circuit is higher.

We have also shown that the improvement that can be obtained by reducing rollbacks and inter-processor communications is limited and does not necessarily reduce the overall performance of the system. Therefore, the focus should be on evenly distributing the workload over the processors so as to keep them as busy as possible. Unless the processors' activity is close to $100 \%$, minimizing communications or rollbacks has a limited effect. 


\section{Chapter 6}

\section{Partitioning and Mapping}

The partitioning problem has generated a great deal of research and has been shown to be NPcomplete. Many heuristics, varying in their degree of complexity and targeting different aspects of the problem have been developed. In the end, most partitioning strategies are based on balancing the load and minimizing communications between processors.

We distinguish partitioning from mapping. Partitioning is defined as the decomposition of a graph into sub-graphs based on some heuristics. In the context of parallel programming applications. the mapping problem is defined as the binding of the logical components of the parallel application program to the physical resources of the target parallel system such that some desired performance criterion is optimized.

In this chapter, we study the impact of different circuit partitioning and mapping strategies on the performance of Clustered Time Warp. The behavior of CTW is analyzed by looking at the load balance, inter-processor communications and rollbacks.

\subsection{Related Results}

\subsubsection{Classical partitioning methods for logic simulation}

The most straighforward partitioning method is the natural partitioning approach [Smit87]. It is often used in comparative studies as a reference. In this approach, gates are assigned to a partition in the sequential order they were read from the netlist input file.

In the gate-level partitioning algorithm [Smit87], the graph of the circuit is traversed and each gate is assigned a level order. Primary input gates have level order 1 . Then the gates which have 
at least one input directly connected to the output of one of the primary gates have level order 2. Similarly, a level order 3 is assigned to the gates which are connected to those having a level order 2. Gates are then partitioned based on their level. Consequently, partitions are likely to group gates whose level order is the same.

Another simple method is the random partitioning approach in which gates are simply assigned to partitions on a random basis. In [Smit8i] and [Brin90], the authors observe that random partitioning results in a good load balance, but high inter-processor communications degrade the performance of the system. Girodias [Giro91] also observes that random partitioning results in higher memory requirements for the Time Warp system. The problem with random partitioning is that the computational locality of the simulation model is not exploited. therefore synchronization overhead and high inter-processor communications can counterbalance the advantage of good balance.

The string partitioning algorithm takes a primary input and builds a string by following one of the paths in the connection graph originating from that input. A string is defined as a set of connected gates with at most one fanin and one fanout included. The algorithm groups strings together to form the partitions while trying to keep the load balanced between the processors. Levendel. Menon and Patel [Leve82] favor string partitioning over other methods since in a circuit signals tend to propagate in parallel. Some circuits such as a pipelined arithmetic unit. have a series of waves of activity. As a consequence, they suggest that the partitioning algorithm must be done along the depth of the circuit. They propose an elegant and simple algorithm based on the string partitioning approach.

The cone partitioning strategy was first introduced by Smith. Underwood and Mercer in [Smit87]. The authors describe two schemes, one based on fanin cones, and one based on fanout cones. In the fanin cone partitioning approach, gates are processed in level order, starting from those assigned at the primary inputs. For each of these gates, the fanin cone is built by following all of the strings which originate from the gate, until the maximum size of the partition block is reached. The partition is then assigned to a processor. The method based on fanout cones is similar but with the difference that primary outputs are considered instead of primary inputs. Even though the concurrency available in a partition resulting from a cone-partitioning 
algorithm is less than that available in a random partition, the partition takes better advantage of the computational locality and consequently, inter-processor communications are reduced.

The advantage of the methods described above are their simplicity, both in terms of implementation and time complexity (all are $O(n)$ ).

Smith, Underwood and Mercer [Smit87] studied the performance of six simple partitioning techniques - natural partitioning, partitioning by gate level, random partitioning, string partitioning, and cone partitioning (based on both input and output cones). Their results show that both random partitioning and string partitioning have about $10 \%$ more concurrency than the cone partitioning methods. On the other hand, cone partitioning results in less inter-processor communication. $80 \%$ less than other techniques. Interestingly, gate level partitioning performs nearly as well as the cone partitioning methods. However, one must recall that the size of the benchmark circuits used in the study were rather small (from 84 up to 3.800 gates) and could have biased the results.

In [Krav88]. Kravitz and Ackland made an empirical study of static partitioning algorithms and observed that the methods which focus on the load-balance of the system generally perform better than those which concentrate on communication costs.

\subsubsection{Adaptive Heuristic methods}

Algorithms which have recently received a great deal of interest is the family of adaptive heuristic techniques such as simulated annealing, probabilistic hill climbing. and sequence heuristics. Given a combinatorial optimization problem. an adaptive heuristic technique first generates an initial solution to the problem. That solution is then perturbed and a new solution is produced. The quality of both solutions is evaluated and compared. Depending on some heuristics, the algorithm chooses either to keep the current solution or to accept the new one. This process is repeated until a terminating criterion is satisfied. Such techniques are said to be adaptive because the parameters of the heuristics can be changed during the execution of the algorithm, either internally by the algorithm itself or externally by a user.

Proposed by Kirkpatrick et al. [Kirk83], simulated annealing is an instance of adaptive heuristic methods and an extension of the Monte-Carlo approach [Metr53]. The goal of the Monte-Carlo 
technique is to find the equilibrium state of a collection of atoms at a given temperature. Starting from a given state, the algorithm generates a new solution by perturbating the state. The new solution is adopted or rejected based on some heuristics. The parameters of the heuristics are then modified if necessary, and the same process is repeated until a termination criterion is satisfied. In simulated annealing, moves that do not reduce the cost are allowed on a random basis, however the probability of such moves to occur decreases over time and is defined by a probability function. For example, Kirkpatrick et al. [Kirk83] used the Boltzmann distribution in their probability function $P(\Delta F)$,

$$
P(\Delta F)=\min \left(1, e^{-\frac{\Delta(F)}{T}}\right)
$$

where $F$ is an objective function which evaluates the quality of a solution, $\Delta(F)$ is the difference of qualities between the current solution and a newly generated solution. and $T$ is the current temperature (which decreases over time).

Witte, Chamberlain and Franklin present in [Witt91] an algorithm using the simulated annealing approach to solve the problem of task assignment. They observe that the performance of the simulation of a logic circuit partitioned with their algorithm is about $22 \%$ better than a random partitioning technique. However, experiments were performed with small models whose size did not exceed 100 elements and where the maximum number of partitions sought was eight. Boukerche and Tropper [Bouk94] make use of simulated annealing to find good sub-optimal partitions of FCFS queueing network models. Performance results were compared with the solution generated by a random partitioning technique. The authors observed a reduction of 25 to $35 \%$ of the running time when their approach is used.

Despite its potential, simulated annealing is an expensive technique and very little can be said about the time needed to reach a good solution.

Random sampling is another adaptive heuristic method but despite its simplicity, it perfors well relative to other approaches [Naha86].

Probabilistic hill climbing, proposed by Romeo and Vincentelli [Rome84] is a variant of simulated annealing in which they allow different temperature update methods and do not necessarily use an exponential function (such as the one used by Kirkpatrick et al. and described above) 
needed to accept or reject a new solution.

In the sequence heuristic approach [Naha85], a bad perturbation of a solution is accepted only if the system has been unable to find a good perturbation over a sequence of attempts.

Few studies have attempted to compare heuristic techniques with other types of algorithms. In [Naha86], the authors made an empirical study of adaptive heuristic techniques including simulated annealing, random sampling, probabilistic hill climbing and the sequence heuristic technique. Each of these strategies was used to solve the optimal linear arrangement (OLA) problem. In this problem, $n$ interconnected circuit elements are given. The objective is to find a linear ordering of these elements so that the number of interconnections that cross between any pair of adjacent elements (called the density) is minimized. In their experiments. the authors generated 10 random instances of 50 circuit elements and 500 nets. Their results show that the sequence heuristic method performed marginally better than did simulated annealing whose performance appeared to be dependent on the choice of the perturbation function. However. the computing time required by adaptive heuristic methods to obtain a good solution was large.

\subsubsection{Other Approaches}

\section{Two-Way Partitioning}

Kernighan and Lin [Kern70] present a two-way partitioning algorithm. In their approach, the graph is initially partitioned randomly into two sets of equal size. Then nodes are interchanged between the two partitions to reduce the number of connections. In [Fidu82], Fiduccia and Mattheyses proposed an optimized version of the algorithm whose complexity is linear for each iteration. However, this improvement is obtained at the cost of a lower quality solution. The authors do not present any empirical evidence regarding the potential improvements of their algorithm over the original version of Kernighan and Lin.

\section{Sensitive Partitioning}

Konas and Yew present in [Kona95] a new partitioning algorithm particularly adapted to logic simulation. In their approach, the authors suggest that the sensitivity of a component (ie: the probability of the component of being active) is almost always inversely proportional to its 
distance from a primary element which can either be a primary input or a synchronous element. The authors suggest partitioning the circuit so that the elements which are likely to be active during the same simulation phase are distributed evenly among the processors.

In their approach, the simulation system is represented by a graph. Logical processes are represented by the nodes of the graph and the edges represent the communications channels between processes. A weight is associated to each node and each edge to represent the processing and communication requirements respectively. The graph is first traversed and each node is assigned a level order (see section 6.1.1). Konas and Yew consider clocked components to be generators of activity waves like primary inputs. As a consequence. during the traversal, such synchronous elements are assigned to level 1. The sensitivity of each element is then calculated. The sensitivity of a component is the maximum probability of one of its inputs changing, which is equivalent to the maximum probability of one of the component's predecessors changing its output. Sensitivities are calculated starting from the primary elements and traversing the graph in a breadth first fashion. Components are then sorted in increasing level order and within each level, components are sorted in decreasing sensitivity order. Based on that sorted list, a greedy algorithm is used to partition the graph. Primary elements are first assigned to different new partitions. Then. the sorted list is traversed and each element is assigned to the partition that will result in the smallest increment in the cost of the simulation graph.

The complexity of the sensitive partitioning algorithm is $O(N P \rho)$ where $N$ is the number of components in the simulated system. $P$ is the number of partitions. and $\rho$ is the average number of predecessors of a component.

The main drawback of this method is that the system is required to know about the probability of each element having its output changed when new input signals are applied. A performance study performed on the largest ISCAS-89 benchmark circuit (containing about 21.000 elements) reveals that sensitive partitioning actually performs better than random partitioning, natural partitioning or string partitioning. 


\section{The Eigenvector Approach}

In [Barn92], Barnes shows that the partitioning problem is equivalent to a matrix approximation problem. Using the eigenvalues of the adjacency matrix, the author presents an algorithm to reduce the cut-set between partitions. However, the worst-case analysis of this approach shows that its complexity is $O\left(n^{3}\right)$. Hadley. Mark and Vannelli propose in [Hadl92] a variant of Barnes' algorithm whose running time is much smaller. When compared to the best netlist partitions obtained by using node interchange heuristics on random initial partitions, the solution generated by their algorithm results in a comparable number or fewer cut-sets.

\section{Abstracting Processes}

Zhang [Zhan92] introduces a new algorithm called Abstracting Processes which transforms any VHDL model [IEEE88] into a modular and hierarchical structure which can be used for the mapping of VHDL blocks onto processors. The author presents several approaches in which the lowest component to distribute onto processors during the distributed simulation is either an entity, a block or a process. In VHDL, an entity denotes an electronic system such as a logic gate. Entities can be grouped into blocks. A process is a language construct that is used to express concurrency. In his research. Zhang suggests using the process as the smallest entity to distribute because it exploits maximum parallelism. Even though no empirical evidence was reported, the author observed satisfactory results.

\section{Corolla partitioning}

In [Dey90, Spor93], the authors describe the corolla partitioning approach based on the analysis of reconvergent fanout. The authors define fanoutstem and reconvergent nodes as follows. If there exists two disjoint paths $P_{i}(x \sim y)$ and $P_{j}(x \sim y)$ whose only common nodes are $x$ and $y$, then the starting node $x$ is called the reconvergent fanout stem and the closing node $y$ is called the reconvergent node.

In the Corolla approach, the circuit is first decomposed into a set of primary stem regions. A primary stem region consists of all the nodes that are located on all the paths from a closing 
reconvergent node to any of its closing reconvergent nodes as well as all the output edges of that node.

Each primary stem region is then partitioned into a set of non-overlapping petals. A petal consists of all the disjoint paths which start at the same fanout stem $x$ and close at the same reconvergent node $y$ with direction of edges ignored.

Because overiapping petals can be generated by different stem regions, those petals are then merged to form corollas. The authors have performed an experimental study of their algorithm in which the quality of partitioning is rated by the number of interconnecting links between the partitions. The authors observe that their approach reduces the cut costs by at least a factor of two when compared to the flip-flop clustering method whose objective is to group into the same partitions the elements which are connected to the same flip-flops. However, the authors also point out that better speedups can be achieved if the signal activity between the gates is taken into consideration during the partitioning, suggesting that dynamic load balancing is a good candidate to improve the overall performance of the system.

\subsection{Discussion}

The partitioning of a logic circuit for distributed simulation may greatly affect the performance of the simulation. If concurrency is not taken into consideration. only a limited amount of parallelism will be extracted from the model. On the other hand. if the partitioning algorithm disregards inter-processor communications, the message traffic might generate such a large overhead that the system will not be very efficient.

In addition to maximizing concurrency and minimizing inter-processor communication, the partitioning algorithm must also produce a solution in which processors have approximately the same amount of work to perform. Unbalanced workload results in a subset of the processors being idle. hence wasting computing resources. Since the problem is NP-complete, heuristics have to be used in order to find a good solution. 


\subsection{Sensitive cone partitioning}

We developed a sensitive version of the cone partitioning algorithm in which synchronous elements such as flip-flops, latches and buffers are considered primary input or output ports. For instance, in the case of the input-cone partitioning, such synchronous elements are considered as primary inputs. Similariy, in the case of the output-cone partitioning, synchronous elements are considered as primary outputs. Introducing sensitivity into the cone partitioning algorithms will likely put logic gates that belong to different combinatorial logic structures in separate clusters.

Figure 6.1 illustrates the effect of sensitivity for the output cone partitioning method. Circles represent logic gates and squares represent synchronous elements in the circuits. The coloring illustrates the different partitions that would result from an output cone partitioning algorithm. whether sensitivity is used or not. The output cone partitioning algorithm starts from a primary output gate $v$ and assigns it to a partition $V_{p}$. All the predecessors of $v$. whose corresponding set is denoted $\Pi[v]$, are also assigned to the same partition $V_{p}$. This procedure is repeated recursively for each element of $\Pi[v]$ until the partition has reached its maximum size, in which case, a new partition $V_{p+1}$ is created. When the algorithm reaches the primary inputs of the circuit, no more predecessors can be found; therefore, the next primary output gate is chosen and the circuit is traversed again. During each traversal, gates that have been already assigned to a partition are ignored.

In the sensitive version, if the predecessor of a gate is a synchronous element (eg: a flip-flop), it is not added to the current partition. Instead, it will be considered just like a primary output and it will be assigned to a partition once the current traversal will be complete.

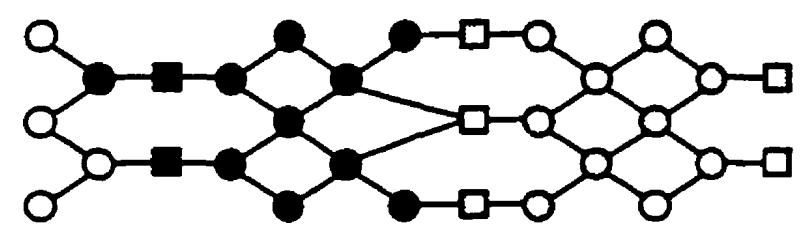

Output Cone

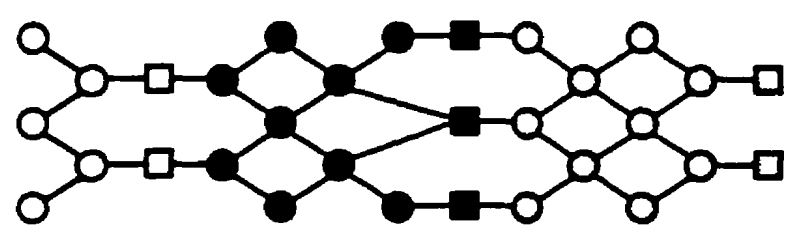

Sensitive Output Cone

Figure 6.1: Effect of introducing sensitivity to an output cone partitioning algorithm 
Figure 6.2 shows the pseudocode of the sensitive output cone partitioning algorithm.

\section{Input}

$G(V, E)$ is the graph to partition.

$\Pi[v]$ is the set of predecessors $\pi[v], \forall v \in V$. size is the maximum size of the partitions.

\section{Output}

$\left\{V_{0}, V_{1}, \ldots, V_{n-1}\right\}$ is the set of partitions.

\section{Temporary}

$S$ is a stack of vertices (first in, last out).

$p$ is the index of the current partition.

\section{begin}

(1) $S \leftarrow \emptyset, p \leftarrow 0$

(2) for each vertex $v \in V$ such that $v$ is a primary output or a synchronous element do

(3) push $v$ onto $S$ endfor

(4) while $S \neq \emptyset$ do

(5) pop $v$ from $S$

(6) if $v$ is not assigned to any partition do

(i) $\quad V_{p} \leftarrow V_{p}+\{v\}$

(8) if $\left|V_{p}\right|=$ size then $p \leftarrow p+1$

(9) for each $u \in \Pi[v]$ do

$$
\text { if } u \text { is not a synchronous element then push } u \text { onto } S
$$
endfor

$$
\text { endif }
$$

endwhile

end.

Figure 6.2: Pseudocode of the sensitive output cone partitioning algorithm 


\subsection{Experiments}

The quality of a partitioning algorithm can be characterized by the three following factors:

1. The amount of concurrency available in the resulting model.

2. The amount of inter-processor communications.

3. The quality of the load balancing.

When optimistic algorithms are considered, other factors might have to be taken into consideration such as rollbacks and memory usage. In this section, we empirically study the effects of different partitioning and mapping strategies on the behavior of Clustered Time Warp.

The partitioning algorithms which we study are: random partitioning, string partitioning, input and output cone partitioning. We also study a sensitive version of both cone partitioning algorithms.

In addition, we study the effect of different mapping strategies such as random mapping, and natural mapping.

\subsubsection{Cut-set size}

The cut-set refers to all the nets that cross the boundaries of the partitions of a model. In the case of a model with uniform behavior and a well distributed workload. a large cut-set size is likely to generate a high communication overhead. However, as we have seen in chapter 2 , digital circuits are characterized by a strong locality of activity. In the following sections, we will show that a small cut-set size does not necessarily result in small inter-processor communication overhead and other factors such as the load balance and the intrinsic properties of digital circuits also have to be taken into consideration when trying to optimize the overall performance of the simulation system.

Each of the partitioning algorithms was run on the four largest circuits (table 6.1) of the ISCAS'89 benchmark suite [Brg189] and the size of the cut-set was measured with different numbers of partitions. 


\begin{tabular}{|c||c|c|c|c|}
\hline Circuit & \# inputs & \# outputs & \# flip-flops & total \\
\hline \hline s38584 & 12 & 278 & 1,452 & 20.995 \\
\hline s38417 & 28 & 106 & 1,636 & 23.949 \\
\hline s35932 & 35 & 320 & 1,728 & 18.148 \\
\hline s15850 & 14 & 87 & 597 & 10.470 \\
\hline
\end{tabular}

Table 6.1: Description of the circuits studied in this chapter

Figures 6.3, 6.4, 6.5 and 6.6 give the relative size of the cut-set obtained by running the different partitioning algorithms on circuits s38584, s38417, s35932 and s15850 respectively (raw data can be found in appendix $C$ ). The relative size of a cut-set is obtained by dividing the actual size of that cut-set by the total number of links in the entire graph. Consider a graph $G(V, E)$ where $V$ is a set of vertices and $E$ is a set of edges. The cut-set $E_{c} \subset E$ is defined such that $\forall e(u, v) \in E_{c}, u$ and $v$ are in different partitions. The relative cut-set size $s_{c}$ is therefore defined as:

$$
s_{c}=\frac{\left|E_{c}\right|}{|E|}
$$

For all of the circuits we observe that the results are approximately the same. In all cases. the partitioning algorithms can be grouped into two categories, those which generate a very large cut-set with a relative size of more than $90 \%$ for 256 partitions (eg: random and string partitioning), and the others which keep the relative cut-set size between 30 and $40 \%$ for 256 partitions. A random partition results with the largest cut-set (a relative size of more than $99 \%$ for 256 partitions) whereas the sensitive output cone partitioning algorithm generates the smallest cut-set, with a relative size of 33 to $38 \%$ for 256 partitions. Also, output cone partitioning performs better than input cone partitioning whether sensitivity is used or not.

The size of the cut-set is not necessarily correlated to the amount of inter-processor communications observed during the simulation of digital circuits. The balance of the system, the intrinsic functional behavior of the circuit, and the concurrency of the simulated model are other factors that have an effect on the actual message traffic between processors generated during a simulation. 

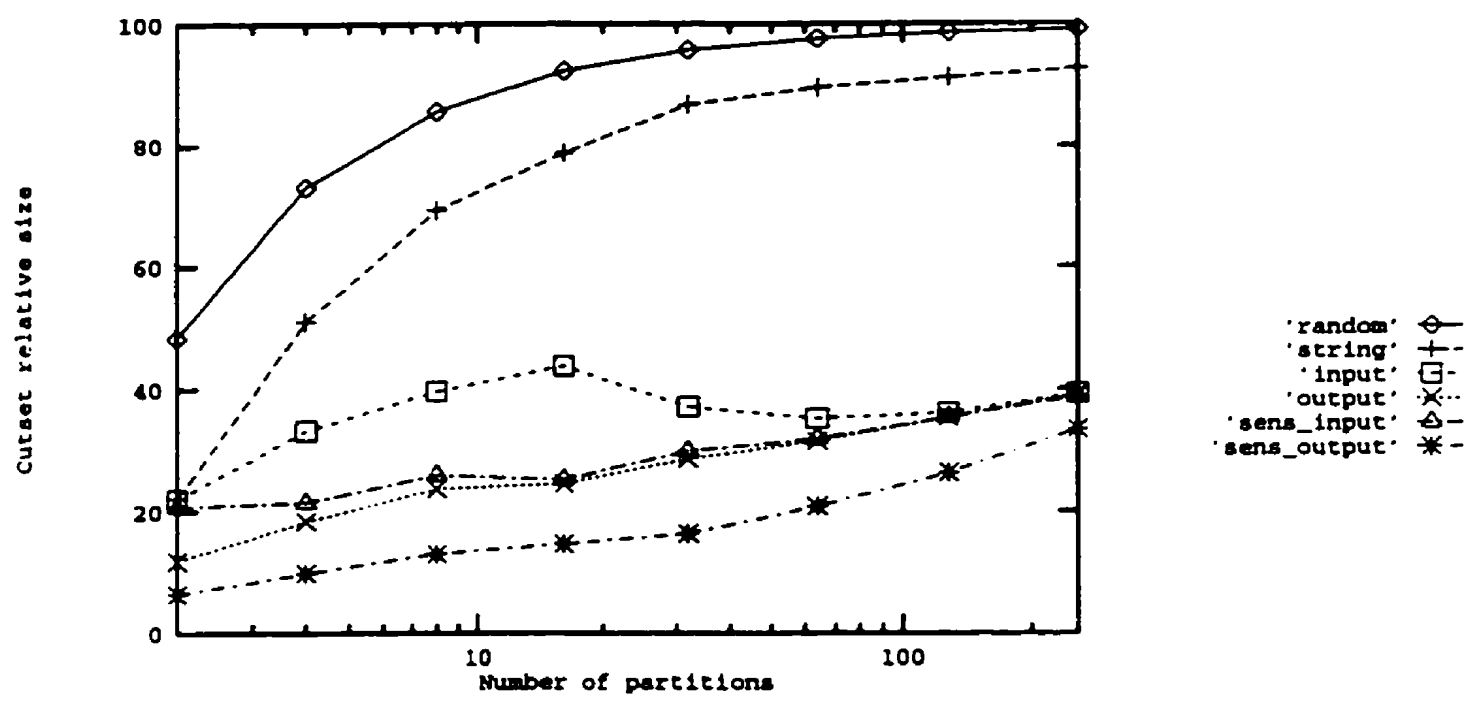

Figure 6.3: Relative size of the cut-set for circuit s38584

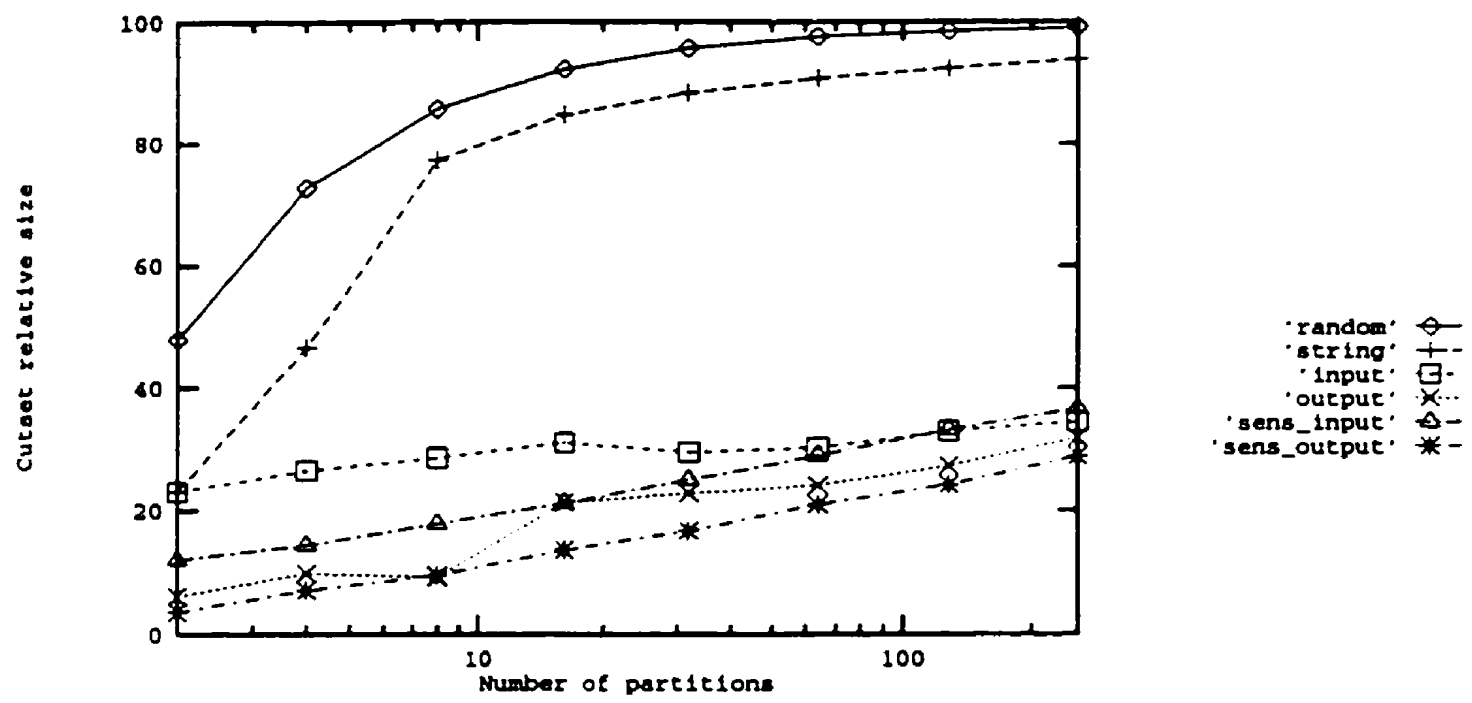

Figure 6.4: Relative size of the cut-set for circuit s38417

In the following sections, we analyze the effect on Clustered Time Warp of the different partitioning strategies using different metrics such as inter-processor communication, rollbacks, system imbalance and simulation time. Using four of the ISCAS'89 benchmark circuits (table 6.1), a series of simulations were run, varying the number of processors from 5 to 25 . A total of 10 clusters per processor was set. Consequently, the cluster size decreased as the number of processors used in the simulation increased. For instance, circuit s38584 contains about 21.000 gates, 

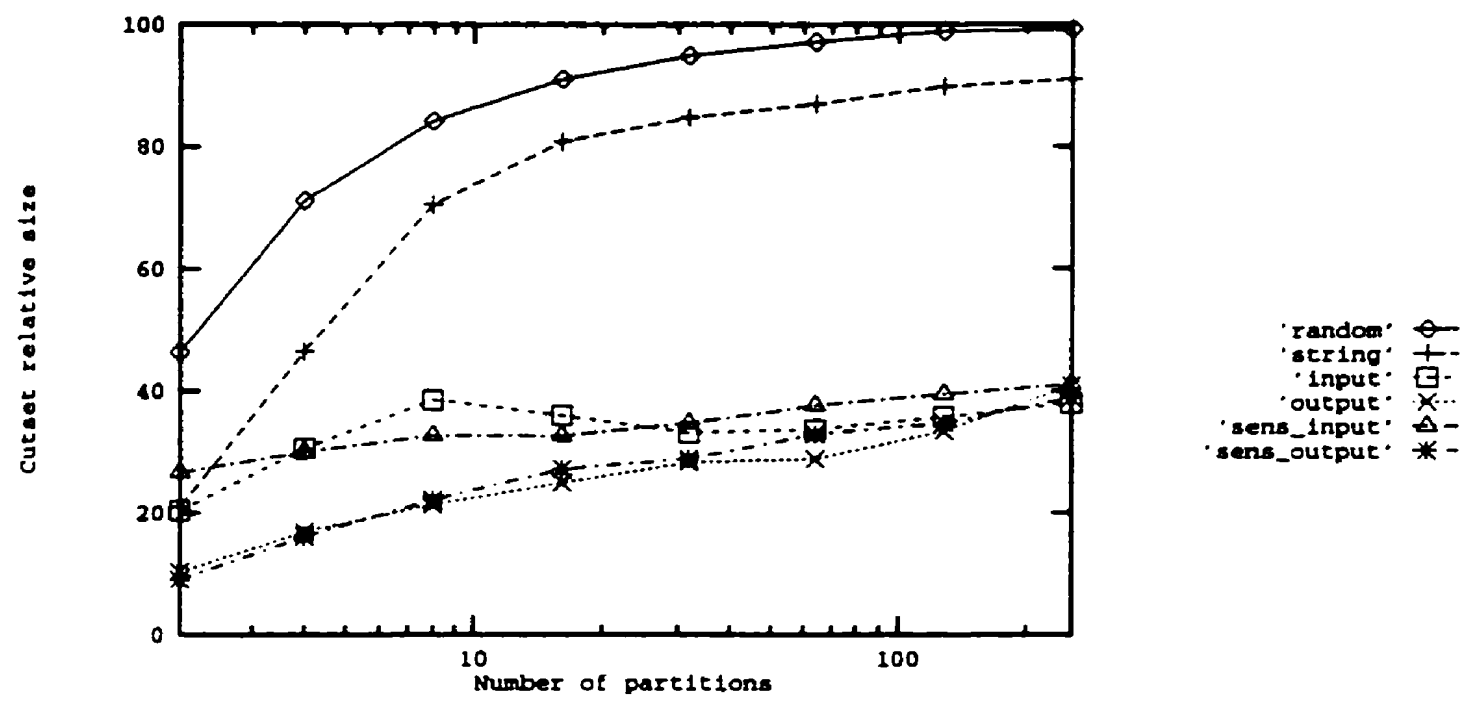

Figure 6.5: Relative size of the cut-set for circuit s35932
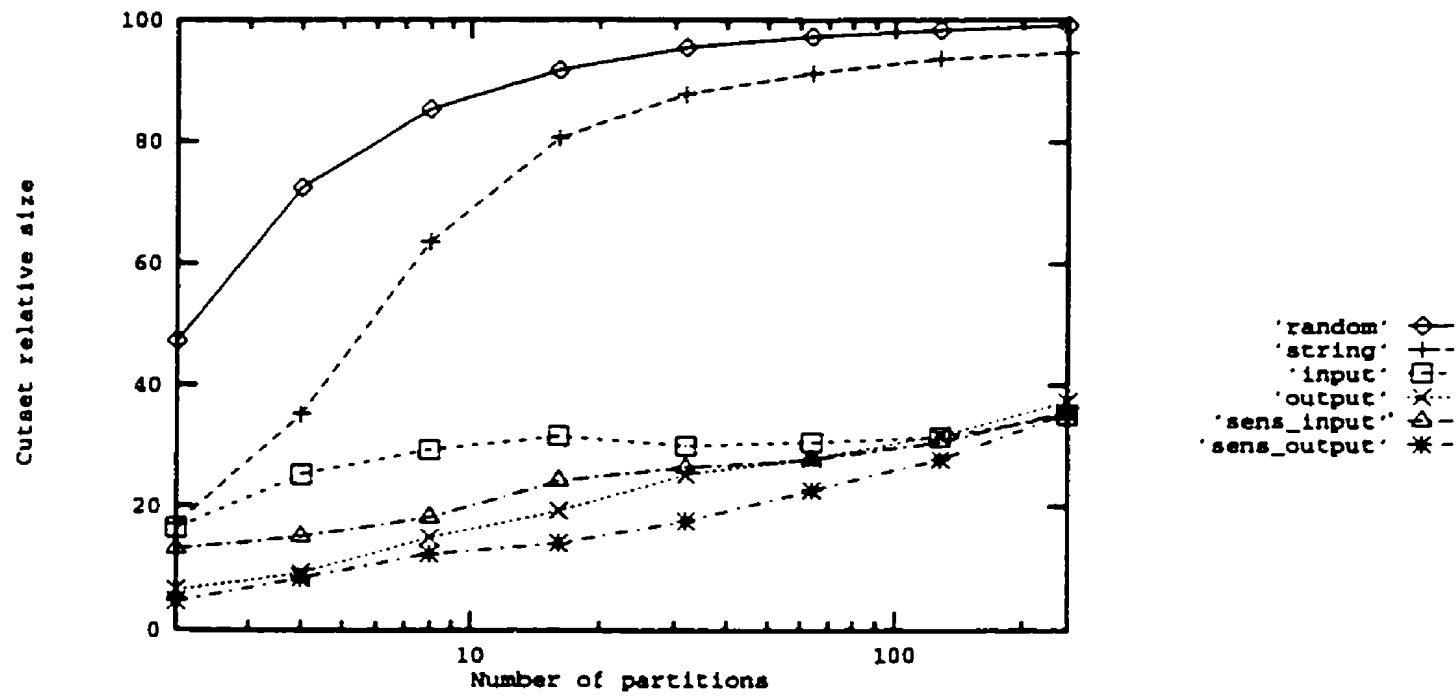

Figure 6.6: Relative size of the cut-set for circuit s15850

hence, when 5 processors were used. the circuit was partitioned into 50 clusters and each cluster contained about 420 gates. Similarly, for 25 processors, the circuit was partitioned into 250 clusters, each of which contains 84 gates. Since results are similar for all circuits. we present in this chapter the data collected from the simulations of s38584. the largest of the four studied circuits. Exhaustive data can be found in appendix $\mathrm{C}$. 


\subsubsection{Inter-Processor communications}

Figure 6. 7 shows the effect of partitioning on the actual amount of inter-processor communications for circuit s38584. We ran a series of simulations on a different number of processors using the Clustered Time Warp simulation system with the LRCC checkpointing method.

It is interesting to observe that the partitioning algorithms that actually performed best when trying to minimize the cut-set size do not necessarily perform as well when we look at the actual message traffic. For example, sensitive output cone partitioning is the algorithm that results in the smallest cut-set. However, experimental results show that the message traffic is actually relatively high. Similarly, the use of string partitioning generally creates a fairly large cut-set. On the other hand, we can observe that the amount of inter-processor communications is lower than most of the other partitioning methods.

These results clearly show that minimizing the cut-set size does not generally minimize interprocessor communications during a simulation. Other factors such as locality and workload distribution also have to be considered.
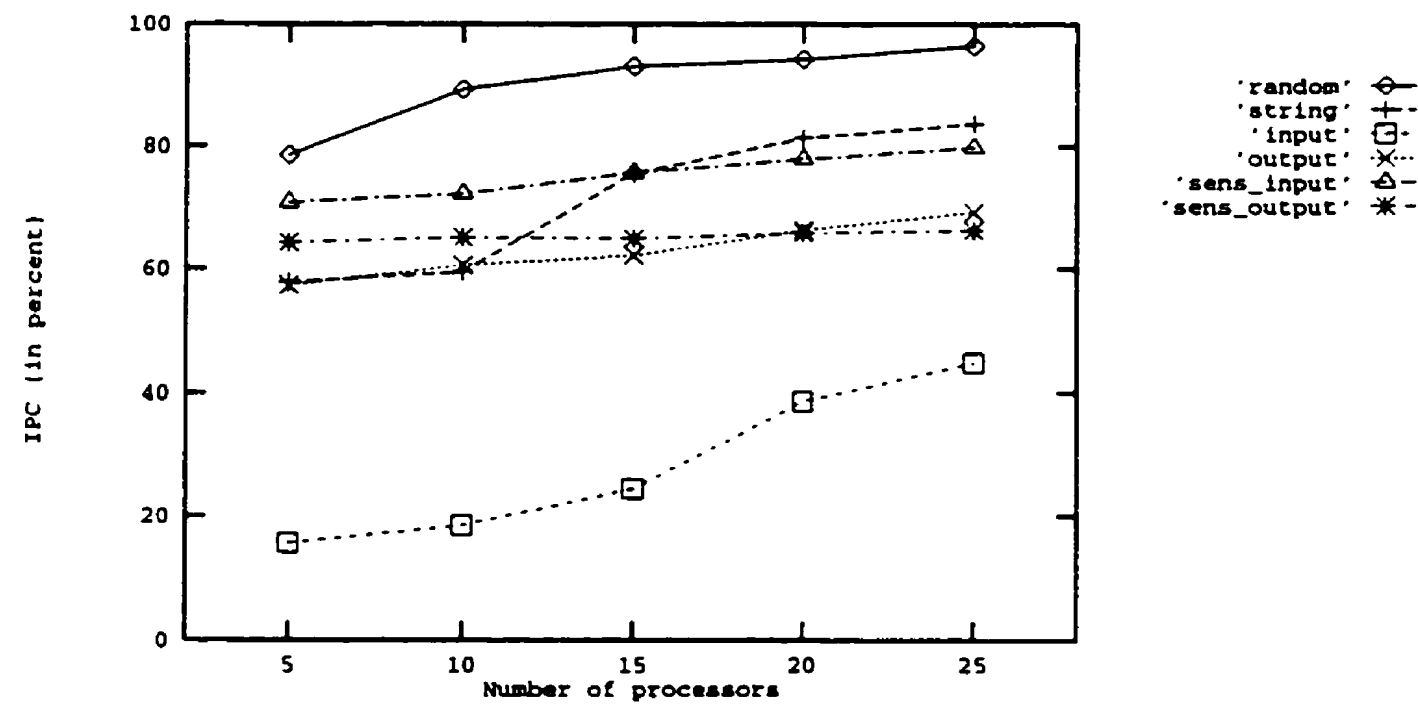

Figure 6.7: Effect of partitioning on inter-processor communications for circuit s38584 


\subsubsection{Rollbacks}

For each simulation, the proportion of rollbacks was measured. In chapter 5 , we defined this proportion as $\rho=E_{\text {cancelled }} / E_{\text {processed }}$ where $E_{\text {cancelled }}$ is the number of events rolled back and $E_{\text {processed }}$ is the total number of events processed. Figure 6.8 shows the proportion of rollbacks for circuit s38584 for each of the partitioning algorithms. We observe that random partitioning performs poorly with $21 \%$ to $31 \%$ of the events being rolled back. This is due to the fact that random partitioning does not try to preserve the structure of the model in any way. On the other hand, the other partitioning strategies perform better than the random approach with output cone partitioning resulting in less than $5 \%$ of the events being rolled back (regardless of the number of processors being used).

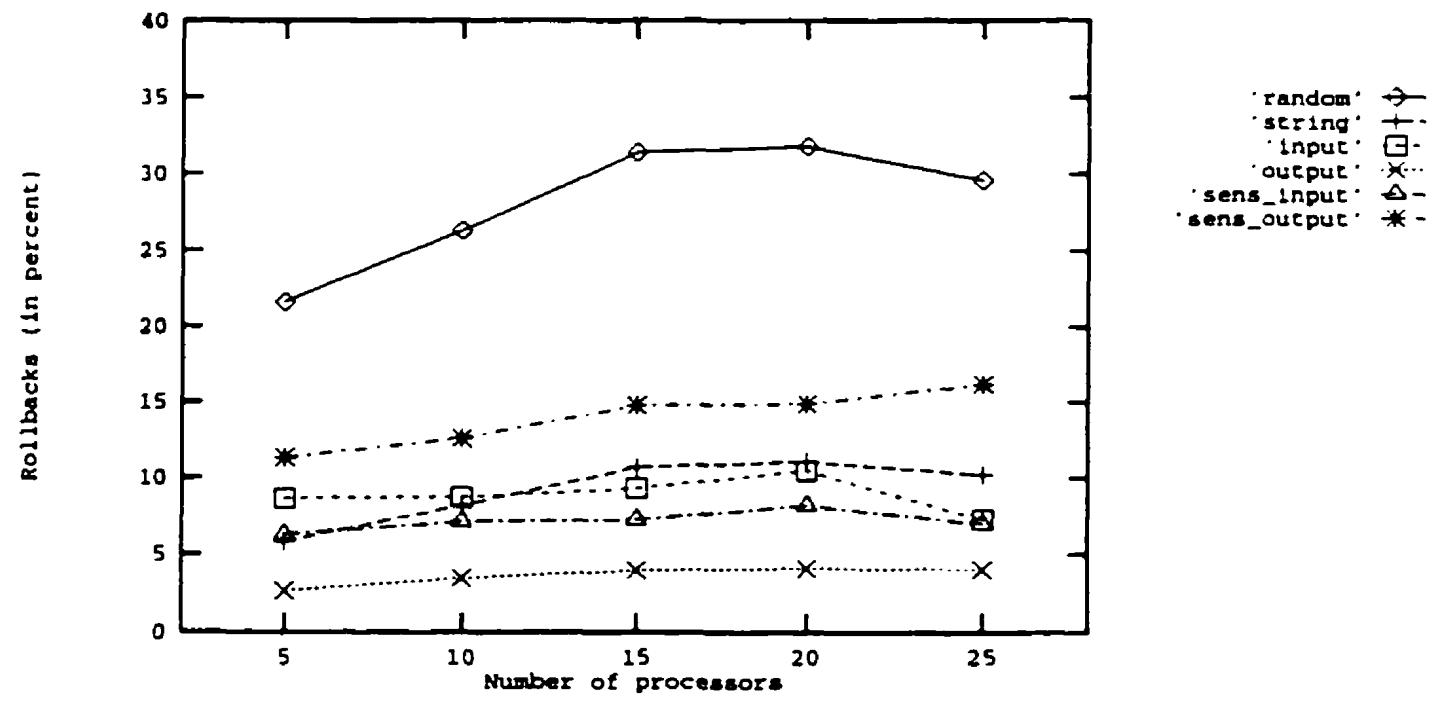

Figure 6.8: Effect of partitioning on the number of rollbacks for circuit s38584

\subsubsection{Workload distribution}

In chapter 5 , we defined the workload distribution $\beta$ as the ratio of the standard deviation of the processor loads to the maximum load observed. This metric estimates the quality of the system balance; the lower that 3 is. the more evenly the load is distributed. Figure 6.9 shows the workload distribution for circuit s38584 for each partitioning algorithm versus the number 
of processors in the simulation. Random partitioning is by far the best with approximately a 3 of $20 \%$ from 10 processors onwards. With the random approach. gates are evenly and uniformly distributed among the processors. therefore the same proportion of active gates is likely to be found in each of these processors. At the other end of the spectrum. a bad workload distribution is observed when the string or an input cone partitioning algorithm is used. 3 varies between 80 and $100 \%$ whether a sensitive approach is used or not. These results suggest that the performance of a simulation system using a model that has been partitioned with either the string or the input cone algorithm is likely to be improved when a dynamic load balancing approach is used. Output cone partitioning results in a fairly well balanced simulation with a 3 varying from 40 to $60 \%$ in most cases.

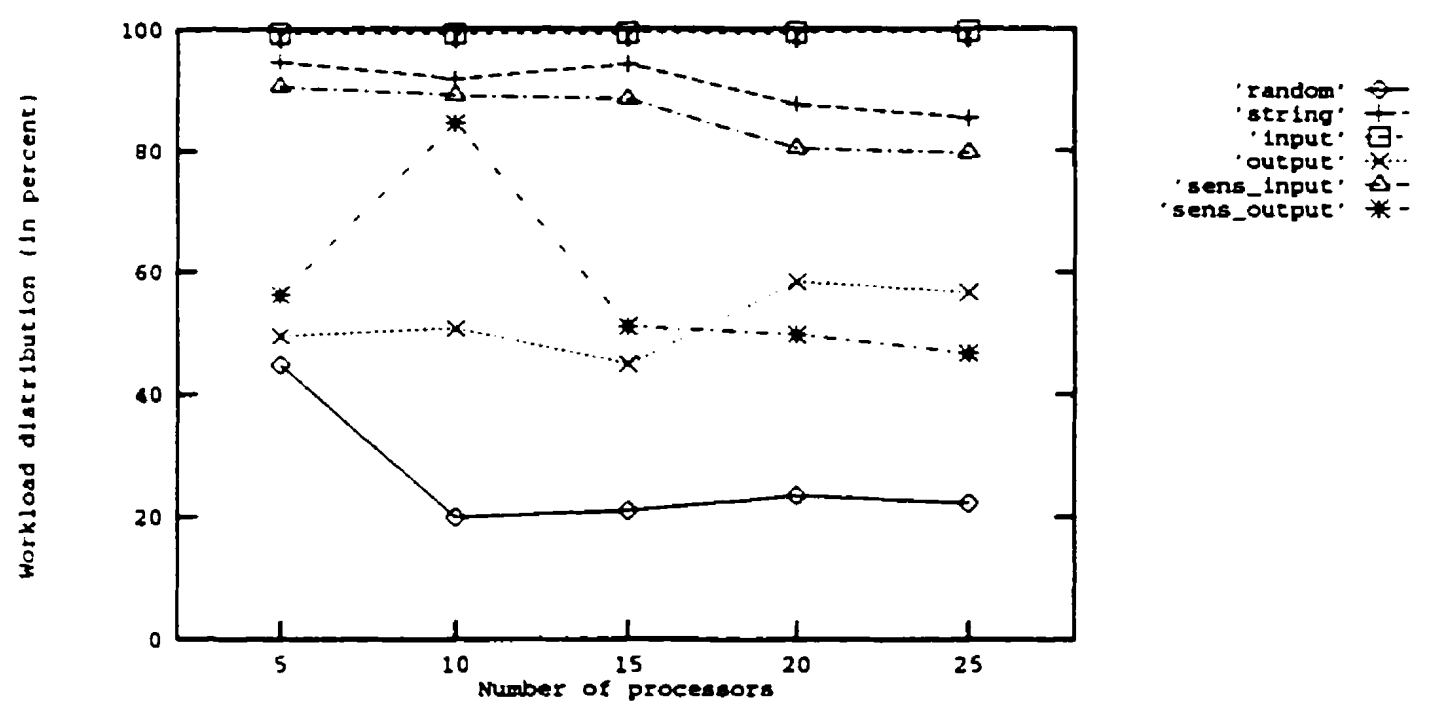

Figure 6.9: Effect of partitioning on the load balance for circuit s38584

\subsubsection{Simulation time}

Simulation time reflects the overall performance of the system. Figure 6.10 shows the time needed by the system to complete the simulation of the circuit s38584, for the different partitioning strategies and varying the number of processors.

We first observe that as the number of processors increases, the partitioning method used 
becomes more important for the performance of the simulation. For instance, when 5 processors are used, the simulation of a model partitioned with the random approach is about $7 \%$ slower than when the sensitive output cone approach is used. However, when 25 processors are used for the simulation, the difference in simulation time increases up to $30 \%$. When a small number of processors are used, random partitioning actually performs relatively well since a lot of parallelism is extracted from the model. However, as the number of processors increases, the communication overhead becomes more important

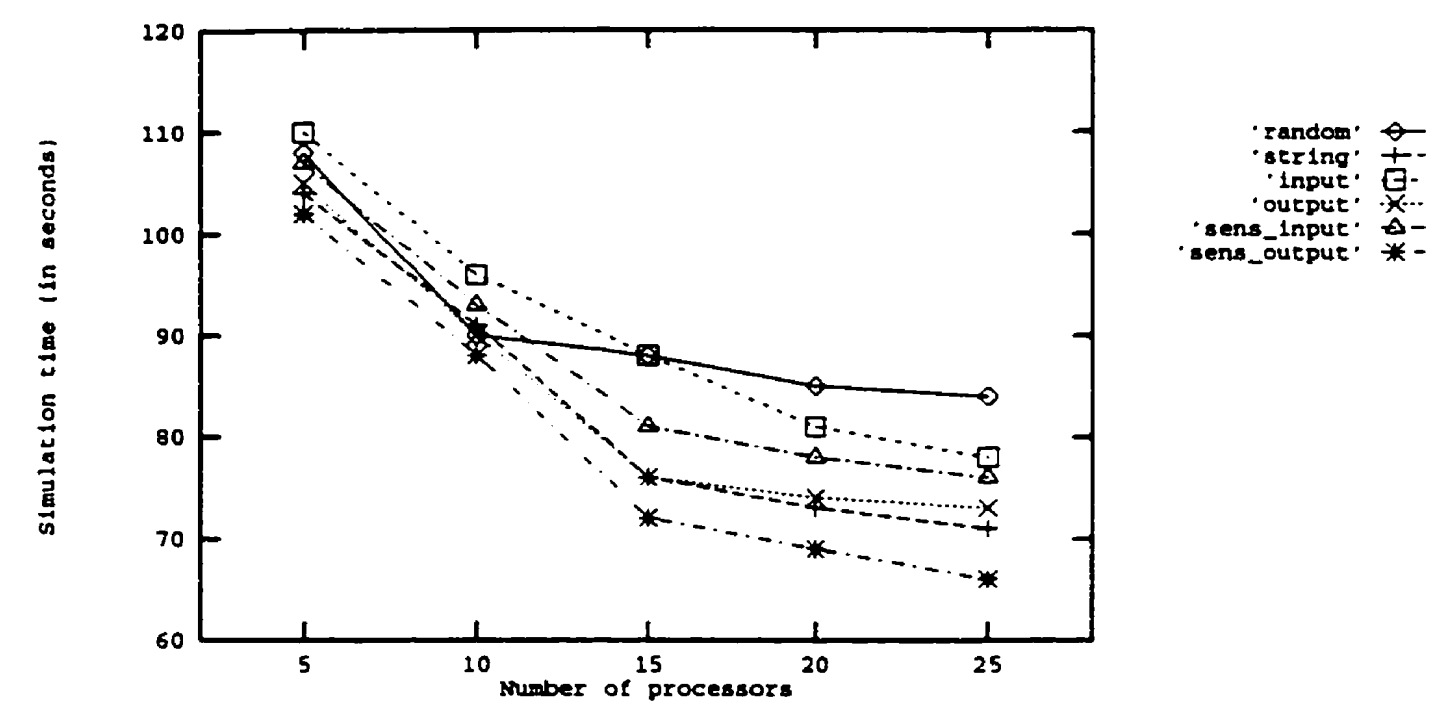

Figure 6.10: Effect of partitioning on the simulation time for circuit s38584

\subsubsection{Mapping}

Mapping binds the logical components of the model to the processors of the target architecture. In our case, the clusters constitute the logical components. We distinguish three types of mapping: vertical natural mapping, horizontal natural mapping and random mapping. In the natural approach, clusters are assigned to processors in the order they were created during the partitioning process of the original model. In the vertical version, the natural mapping strategy assigns as many clusters as possible to a processor before selecting the next processor. If $P_{i}$ is the processor hosting cluster $C_{j}$, then $i=\left\lfloor j / n_{c}\right\rfloor$ where $n_{c}$ is the number of clusters to be assigned 
to each processor. In the horizontal version, each time a cluster is assigned to a processor $P_{i}$. the next processor $P_{i+1}$ is selected to receive the next cluster. In general, cluster $C_{j}$ is assigned to processor $P_{i}$ such that $i=j-\lfloor j /|\Pi|\rfloor$ where $\Pi$ is the set of processors. In the random approach. clusters are simply randomly assigned to the physical units of the system. Figure 6.11 shows an example the natural and the random mapping strategies. A graph containing 40 nodes is first partitioned into 10 clusters using an input cone approach. Each of the clusters is then mapped to a set of five processors.
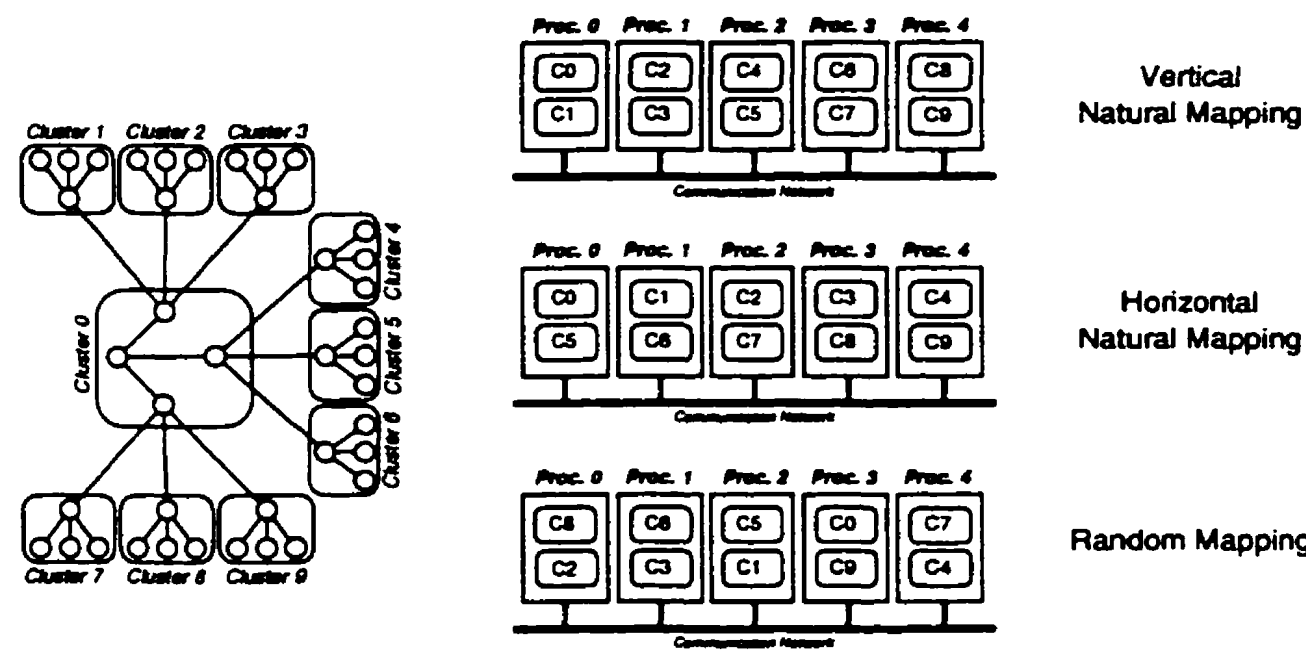

Random Mapping

Figure 6.11: Example of the Natural and the Random mapping strategies

Vertical natural mapping will likely reduce inter-processor communications since message traffic tends to be heavier between adjacent clusters. On the other hand. the assignment resulting from this type of mapping might be more sensitive to long feedback loops because clusters far away from each other in the model graph will tend to have loosely coupled local times. The main advantage of the horizontal natural mapping strategy is to group together on the same processor clusters which are far apart in the graph. Because the minimum message timestamp scheduling policy, these clusters can not directly roll back each other. therefore the sensitivity of the simulation system to large rollbacks is reduced. However, the communication overhead will likely to be larger than in the case of vertical natural mapping because the clusters which are adjacent in the model graph will be assigned to different processors. Random mapping is a 
trade-off between the reduced communication overhead induced by vertical natural mapping and a better overall synchronization of the processes in the horizontal natural mapping. In addition, the simulation system is more likely to be well balanced with random mapping as we have seen in section 6.4.4.

The mapping strategy does not have any strong effect when the original model is partitioned randomly. Whether natural of random mapping is used, the final assignment of nodes to the processors will still be random. However, the mapping of the clusters can affect the performance of the simulation system when other partitioning algorithms are considered. In the results presented in the previous sections of this chapter, a vertical natural mapping was used. In this section. we present the results obtained from using horizontal natural mapping and random mapping as well for the simulation of the four circuits on 20 processors.

\section{Inter-processor communications}

For each of the six partitioning algorithms studied. we ran three simulations on 20 processors each of which used a particular mapping (either random, vertical natural. or horizontal natural mapping). In all cases, the circuit was partitioned into 200 clusters of equal size ( 10 clusters per processor). Data can be found in appendix $C$ in tables C.6, C. $i$ and C.8.

Figure 6.12 shows the effect of mapping on inter-processor communications for circuit s38584. As expected, the mapping strategy has no effect when random partitioning is used. In all cases, at least $95 \%$ of the messages are sent over the network. When string partitioning is used, we observe

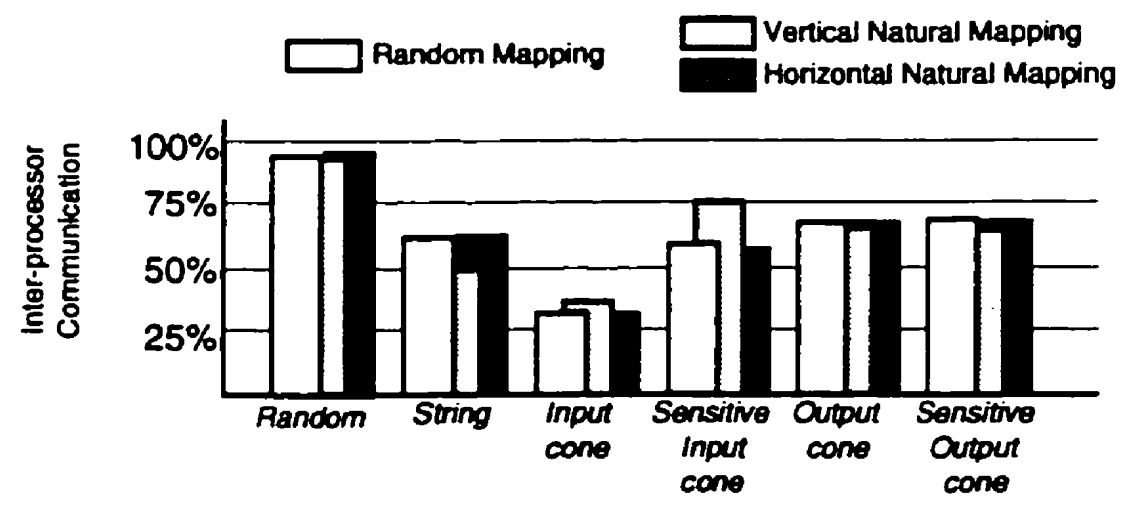

Figure 6.12: Effect of mapping on the interprocessor communications (circuit s38584) 
that the communication overhead is substantially reduced when vertical natural mapping is used. About $13 \%$ less messages are sent over the network. However, the vertical natural mapping strategy creates about $17 \%$ more communication overhead than any of the two other mapping strategies when sensitive input cone partitioning is used. The same effect can be observed for the input cone partitioning algorithm, but to a lesser extent. We do not observe any substantial changes in the communication overhead when output cone partitioning is used (with or without sensitivity).

In general, irrespective of the mapping strategy used, input cone partitioning is the algorithm which minimizes inter-processor communications (30\% of the messages are sent over the network). The largest communication overhead was observed when random partitioning was used (at least $95 \%$ of the messages sent over the network).

\section{Workload distribution}

Figure 6.13 shows the workload distribution observed when using the six partitioning algorithms with different mapping strategies. Except for the random partitioning algorithm where the mapping strategy has very little impact, vertical natural mapping creates in general the most unbalanced simulations. especially in the case of the string partitioning technique.

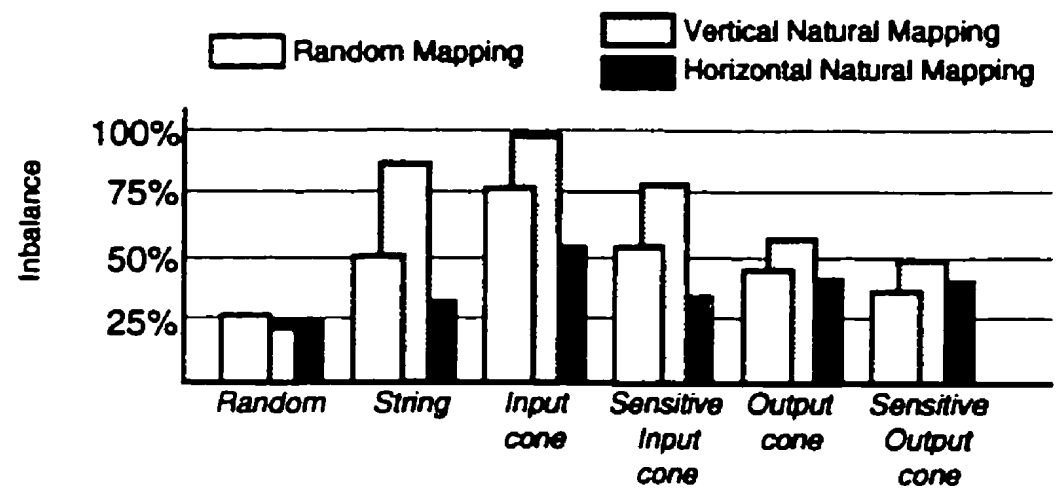

Figure 6.13: Effect of mapping on the workload distribution (circuit s38584) 


\section{Rollbacks}

Figure 6.14 shows the ratio of rolled back events for each partitioning algorithm and each mapping strategy. We observe that random and horizontal natural mapping generate approximately the same amount of rollbacks in all cases except when the output cone partitioning algorithm is used, in which case there are about $5 \%$ less rollbacks for random mapping. However. vertical natural mapping generally performs much better than any of the two other strategies. These observations do not apply when random partitioning is used where the mapping has very little effect on the behavior of the simulation.

In all cases, we can see that when we compare the results of the different mapping strategies for each partitioning techniques. a better balance generates more rollbacks. This is due to the fact that when a system is unbalanced, active processes tend to be located on the same processor. Because a minimum timestamp message scheduling is used. processes are then better synchronized and are less likely to have their local virtual clocks far apart from each other.

In general, output cone partitioning with vertical natural mapping results in fewer rollbacks than any other method.

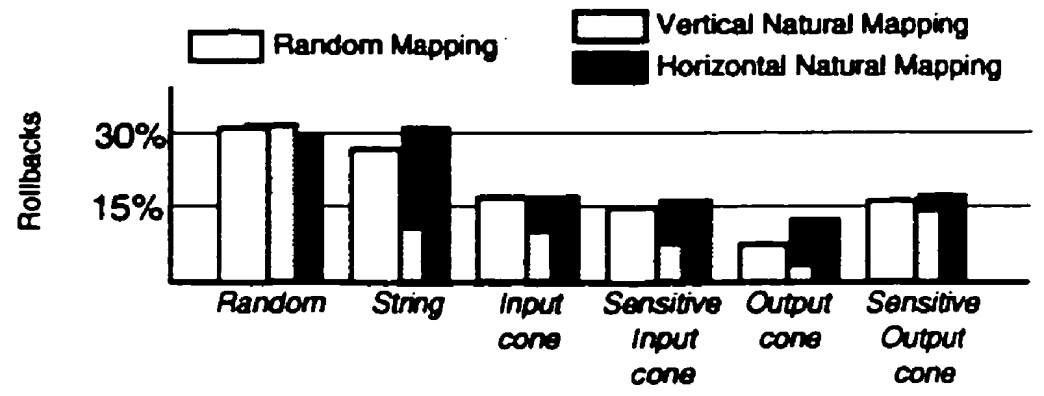

Figure 6.14: Effect of mapping on the rollbacks (circuit s38584)

\section{Simulation time}

The simulation times are presented in figure 6.15. We observe that in general, the partitioning has a more important effect on the simulation time than the mapping. Whatever mapping strategy is used, no substantial improvement of the overall performance of the system could be observed. 
This suggests that the improvements due to the reduction of rollbacks when vertical natural mapping is used are counterbalanced by the loss of concurrency due to the load imbalance.

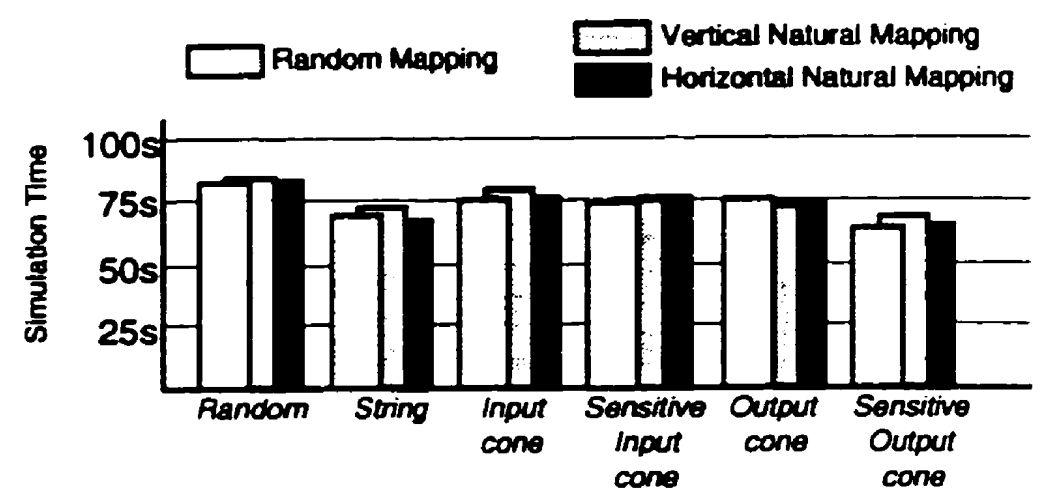

Figure 6.15: Effect of mapping on the simulation time (circuit s38584)

\subsection{Summary}

In this chapter, we have investigated the impact of different partitioning algorithms and mapping strategies on the behavior and the performance of the Clustered Time Warp algorithm.

We first showed that sensitive output cone is the most successful partitioning algorithm when trying to minimize the size of the cut-set. However, empirical results show that the input cone partitioning algorithm actually generates the smallest inter-processor communication overhead. This suggests that partitioning algorithms whose goal is to minimize the number of cut-nets between the partitions may not necessarily translate into a reduction of the actual communication overhead.

We also observed that load imbalance and rollbacks are strongly correlated. For instance, a model partitioned randomly generates a relatively high amount of rollbacks. On the other hand, the system is very well balanced, which greatly favors concurrency.

We have shown that the mapping strategy has also an impact on the behavior of the simulation system when imbalance and inter-processor communications are analyzed. However, no substantial improvements of the overall performance of the simulation system was observed when using 
different mapping strategies, suggesting that the partitioning of a model is a more important factor.

The results presented in this chapter are not necessarily applicable to other architectures. Factors such as communication bandwidth, memory, and processor speed can affect the behavior of the simulation system.

In view of the complex effects caused by trying to either minimize rollbacks or to balance the load or to reduce inter-processor communications via our heuristics. we conclude that a possible approach to improving the performance of a parallel simulation is by a dynamic algorithm. 


\section{Chapter 7}

\section{Scalability and Stability}

\subsection{Related Results}

Very little has been written regarding the scalability of optimistic algorithms. Despite the simplicity of the behavior of each individual process, the behavior of a simulation model composed of several thousands or even millions of processes is hardly predictable and relates more to the field of emergent systems [Farbi3. Hube93] and chaos theory [Wald92. Kell94]. New properties may emerge from such large systems and the fact that pure optimistic techniques do not bound the individual processes in their computation may create situations in which the simulation may become out of control and inefficient.

In [Wiel89], the authors analyze the performance of Time Warp for a combat simulation model running on the Caltech/JPL Mark III Hypercube. Despite the fact that a speed up of 27 was measured with 64 processors. the number of antimessages and rollbacks increased substantially as the number of processors increased, while the size of the actual simulation was constant. For example. for the same simulation which generated around 450.000 events on a single processor. the total number of rolled back events was about $2 \bar{i} .000$ on 4 processors and more than 300.000 with 64 processors. hence increasing the system overhead and reducing its overall performance.

Steinman [Stei92] reports results of SPEEDES, a parallel discrete event simulation system developed at the Jet Propulsion Laboratory. The author describes a proximity detection simulation in which about 1,000 aircrafts are flying randomly around the globe and 259 random sensors perform their proximity detection. In one experiment. Steinman observes a rare example of rollback explosions during which the number of cancelled events becomes higher than the number 
of processed events. Even though all processors are busy doing computation. fewer events get committed and the advance of the Global Virtual Time decreases and stagnate.

Lubachevsky, Schwartz and Weiss [Luba89] analytically evaluate optimistic simulations using a model in which the $j$-th rollback of generation $i$ (we denote as $\rho_{j}^{i}$ ) is represented by a node $(i, j)$ of a tree. The children of node $(i, j)$ models the rollbacks induced by $\rho_{j}^{i}$ and $W(i, j)$ is a random variable which models the change in rollback size between node $(i, j)$ and its ancestor $(i-1, k)$. For example, if node $(i, k)$ rolls back $t$ units of time, then its child node $(i, j)$ rolls back $t+W(i, j)$ units. The values of $W(i, j)$ are identically distributed with the probability function $F(t)$. The authors also define $b$ to be the branching factor which represents the average number of children for all nodes in the tree and $h$ to be a measure of the the tail of the change in rollback size as one moves down the tree. Formally, $h$ is defined as follows:

$$
h=\sup _{-\infty<0<\infty}-\log E e^{\theta W(1.1)}
$$

Based on this model. the authors define stability such as if $b<e^{h}$. the rollback-based simulation is efficient, otherwise if $b>e^{h}$, there exists a probability that unbounded cascading rollbacks may develop, making the simulation system inefficient. They also show that an algorithm may be efficient for a particular set of parameters and inefficient for a different set. This suggests. predicting the efficiency of a large simulation through small test runs can be misleading. Finally, they present a new parallel discrete event simulation algorithm called filtered rollback. In their approach, the execution of certain events is postponed in order to keep the number of rollbacks under control.

In the following sections, we use the terms stability and scalability. We say that a Time Warp system is unstable if the number of rolled back events during a simulation run is not bounded, hence making it impossible for the simulation to terminate in a finite amount of time. Furthermore, we define the scalability of a Time Warp based system to be the rate at which the proportion of rolled back events to committed events increases relative to the size of the simulated model. 


\subsection{Time Warp Hazards}

In addition to large memory consumption, Time Warp practitioners who deal with rather large simulation models have to face other types of problems related to the unpredictable behavior of Time Warp in which the number of rollbacks may become uncontrollable. In this section, we will describe examples in which Time Warp does not scale and may even become unstable.

\subsubsection{Echoing}

In [Luba89], the authors give an interesting and simple example of echoing in which the number of rollbacks increases in proportion to the square root of the physical time.

Consider a physical system composed of three physical processes. A. $B$ and $C$ (see figure $i .1$ ). Upon the arrival of a message from $B$. node $A$ takes $u$ units of real time to process that message and to send back a reply to $B$. If $A$ does not receive anything from $B$ for $2 u$ units of real time. node $A$ sends a message to $C$. Therefore, the system behaves in the following way: $B$ sends a message to $A$ at $t_{r}=u$. $A$ replies back to $B$ at $t_{r}=2 u, B$ replies to $A$ at $t_{r}=3 u$. and so on. In any case, $C$ does not receive any message from either $A$ or $B$.

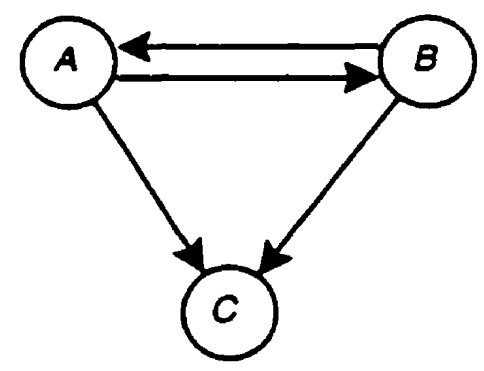

Figure 7.1 : Connection graph of the nodes involved in the echoing phenomenon

Now consider a logical system modelling the physical system described above and composed of three logical processes $L P_{A}, L P_{B}$ and $L P_{C}$ which model the physical processes $A, B$ and $C$. Each logical process is hosted by a different processor. Suppose that the simulation of the processing of a message and the sending of a reply takes one unit of real time regardless of the source or the destination of that message. Furthermore, suppose that sending an antimessage during a rollback operation takes also one unit of real time. 
First, at real time $t_{r}=0, L P_{A}$ receives message $m_{B A_{0}}$ timestamped 0 from $L P_{B}$. therefore, $L P_{A}$ processes $m_{B A_{0}}$ and sends a reply $m_{A B_{0}}$ timestamped $u$ to $L P_{B}$ at $t_{r}=1$. However, since $L P_{B}$ has not received anything from $L P_{A}$ during the real time interval $\{0.1\}, L P_{B}$ sends a message $m_{B C_{0}}$ timestamp $2 u$ to $L P_{C}$.

Now $t_{r}=1$ and $L P_{B}$ receives $m_{A B_{0}}$ from $L P_{A}$. Since the timestamp of the message is $u . L P_{B}$ rolls back to simulated time 0 and sends the antimessage $\bar{m}_{B C_{0}}$ to $L P_{C}$. In the meantime. $L P_{A}$ sends $m_{A C_{0}}$ timestamped $3 u$ to $L P_{C}$.

At $t_{r}=2, L P_{B}$ has rolled back, processes $m_{A B_{0}}$ timestamped $u$ and sends the reply $m_{B A_{1}}$ to $L P_{A}$ with timestamp $2 u$. Meanwhile. since $L P_{A}$ has still not received anything from $L P_{B}$, a new message $m_{A C_{1}}$ with timestamp $5 u$ is sent to $L P_{C}$. At this stage of the simulation. $L P_{A}$ has sent two incorrect messages to $L P_{C}$.

At $t_{r}=6, L P_{B}$ has sent 3 wrong messages to $L P_{C}$.

Successively, one after the other, $L P_{A}$ or $L P_{B}$ sends messages to $L P_{C}$ while the other is busy rolling back. For example. at $t_{r}=6 . L P_{B}$ has sent 3 wrong messages to $L P_{C}$. As the physical time progresses, the amplitude of the rollbacks increases without bound, and the simulation system ends up being completely inefficient.

This example is based on a series of very strong assumptions concerning the implementation and the model. and it is very unlikely to happen, especially in the case of digital circuit simulations. Nevertheless, this example shows that there exists a possibility of instability with Time Warp.

\subsubsection{Cascading Rollbacks}

A different and a more common problem of Time Warp is the risk of cascading rollbacks. Consider the model depicted in figure 7.2 .

Suppose $L P_{A}$ sends at physical time $t_{r}=0$ a message timestamp $u$ to both its children $L P_{B}$ and $L P_{C}$. Similarly, at $t_{r}=1$. both $L P_{B}$ and $L P_{C}$ send a message timestamped $2 u$ to their children. And so on until the bottom of the tree is reached. Suppose now that $L P_{A}$ receives a straggler timestamped $v$ such that $v<u$. In this case, $L P_{A}$ has to send antimessages to $L P_{B}$ and $L P_{C}$ which will also send antimessages to their own children, and so on. In this example, we can 


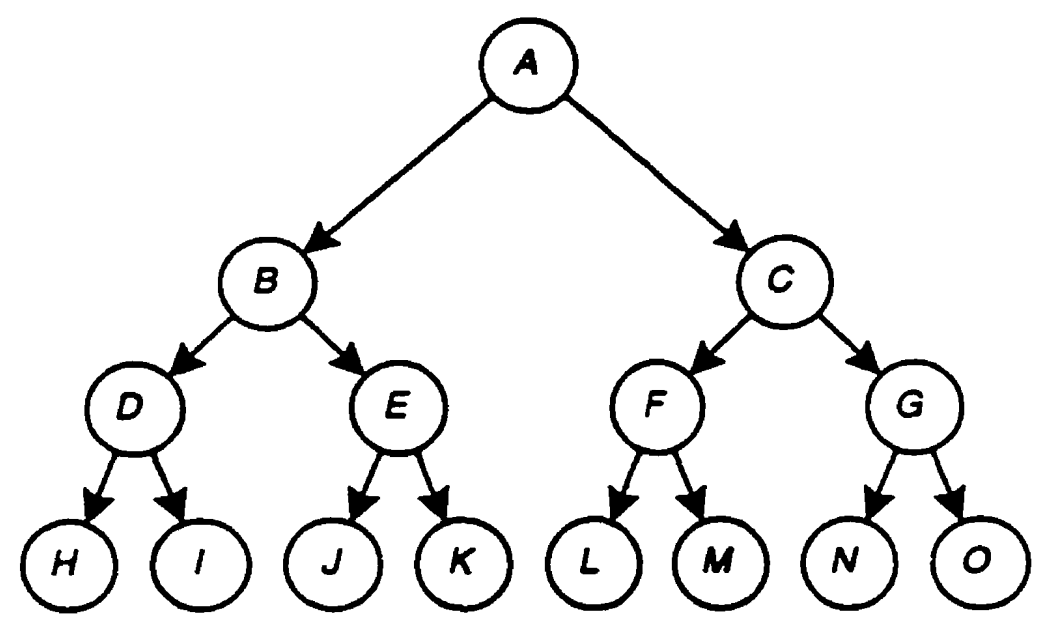

Figure 7.2: Connection graph of the nodes involved in the cascading phenomenon

characterize two types of activity: a computation wave and a cancellation wave. A computation wave is composed of normal messages that trigger the logical processes to reevaluate the model. A cancellation wave is composed of antimessages and is associated to a computation wave. Its objective is to cancel the work performed by the logical processes and which have been induced by the associated computation wave. Both waves alternate with each other and in normal conditions. the cancellation wave catches up with the computation one, in which case they annihilate with each other.

The time needed for the cancellation wave to annihilate its associated computation wave is critical to the performance of a Time Warp based system. In our example. if antimessages do not travel fast enough down the tree to reach their peer messages, the processors might end up wasting all their resources (CPU time and memory) doing wrong computation and cancelling it soon after.

Cascading rollbacks do not make Time Warp unstable since the rollback amplitude is bounded, however, a simulation system that is prone to such a phenomenon is unlikely to be very scalable. As the size of the simulation grows. the overhead of the Time Warp based algorithm is likely to grow at a faster rate. 


\subsubsection{Dog chasing its tail}

This phenomenon is mainly due to the presence of cycles on the connection graph of the simulated model. Consider the model depicted in figure 7.3 in which 5 logical processes are connected into a ring topology.

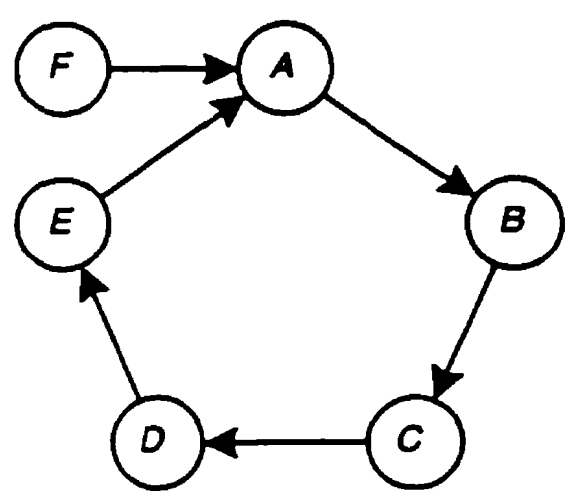

Figure 7.3 : Connection graph of the nodes involved in the 'dog chasing its tail" phenomenon

Suppose the simulation starts with $L P_{F}$ (the only source in the graph) sending one or more messages to $L P_{A}$. A computation wave $W_{0}$ is therefore going to travel around the ring and come back to $L P_{A}$. Because the model is assumed to be stable, $W_{0}$ will be stopped by one of the processes and die. In this example, we assume that $W_{0}$ dies when it comes back to $L P_{A}$.

Suppose now that before $W_{0}$ dies out, $L P_{F}$ sends another message to $L P_{A}$ which happens to be a straggler. In this case. $L P_{A}$ needs to send antimessages to $L P_{B}$ to cancel the wrong computation. hence a cancellation wave $\overline{W_{0}}$ is generated, as well as a new computation wave $W_{1}$ induced by that the straggler received by $L P_{A}$. At this stage of the simulation. the system contains the first computation wave $W_{0}$, followed by $\overline{W_{0}}$ (its associated cancellation wave), and a new computation wave $W_{1}$.

Processes which are traversed by $W_{0}$ result in a state that belongs to a different history path than the state of the processes which result from the processing of $W_{1}$. This is not a problem until $W_{0}$ completes its first cycle at which stage, it reaches process $L P_{A}$. If no straggler had been received by $L P_{A}$, the state of that process would have resulted from the processing of $W_{0}$, and the computation wave would have normally died at $L P_{A}$. But since the process has received a straggler, its state results from the processing of $W_{1}$, and because $W_{0}$ belongs to a different 
history path, the processing of $W_{0}$ by $L P_{A}$ can result in an inconsistent state. Hence, there exists a possibility for $W_{0}$ not to be stopped at $L P_{A}$ and continue its journey towards the next process.

As a consequence, there exists a possibility for the computation wave $W_{0}$ to stay alive until the cancellation wave $\overline{W_{0}}$ catches up. This problem is known as the dog chasing its tail phenomenon.

Even if the simulated model is stable, we have shown that a Time Warp based system can become unstable if it has not been designed properly. If antimessages are not given a higher priority or if their processing time is larger than the processing time of normal messages, the cancellation waves will not be able to catch up with the computation waves. However. even if antimessages travel faster than messages, the time needed for a wrong computation to be cancelled is critical to the performance of a simulation system. The faster a wrong computation wave is stopped, the less resources are wasted.

\subsubsection{Discussion}

Nothing prevents any of the phenomena described above from occurring in a digital logic simulation. although echoing would be an exceptional occurrence since it is based on strong assumptions for both the model and the implementation of the simulation system.

Cascading rollbacks is a real danger because digital circuits are designed in such a way that the number of gates triggered by the change of a signal tends to grow rapidly (see chapter 2). A circuit with a large fanout is likely to be prone to rollbacks more than a circuit with a small fanout. Also. the presence of a large number of cycles in digital circuits favors greatly the dog chasing its tail phenomenon.

Even though it is unlikely to observe instability, the scalability of a Time Warp based algorithm is a very important factor if large simulation models are considered.

The original checkpointing technique of the Clustered Time Warp algorithm (Clustered Checkpoint - Clustered Rollback or CRCC) is characterized by the fact that cancellation is performed at a higher level (clusters) than the computation (processes). This singularity allows the cancellation wave to travel much faster than the computation waves depending on the size of the clusters. Consider the cluster depicted in figures 7 .ta and $7.4 \mathrm{~b}$. The progress of a normal computation wave throughout the cluster is shown in figure $7.4 \mathrm{a}$. Messages are received by the 
Cluster Environment (CE) which updates the timezone table (see chapter 3) and then forwards the messages to the destination process $L P_{1}$. Changes propagate to process $L P_{20}$ which gives the $\mathrm{CE}$ messages that have to be sent to processes located in different clusters. The Cluster Environment keeps a copy of these messages and send them out. Figure $i .4 b$ depicts the journey of a cancellation wave. Upon the arrival of antimessages, the CE immediately sends out the necessary antimessages, directly bypassing the 20 processes. In the case of the Time Warp algorithm. both the computation and the cancellation waves use exactly the same path. hence making it more difficult for the latter to catch up with the former.
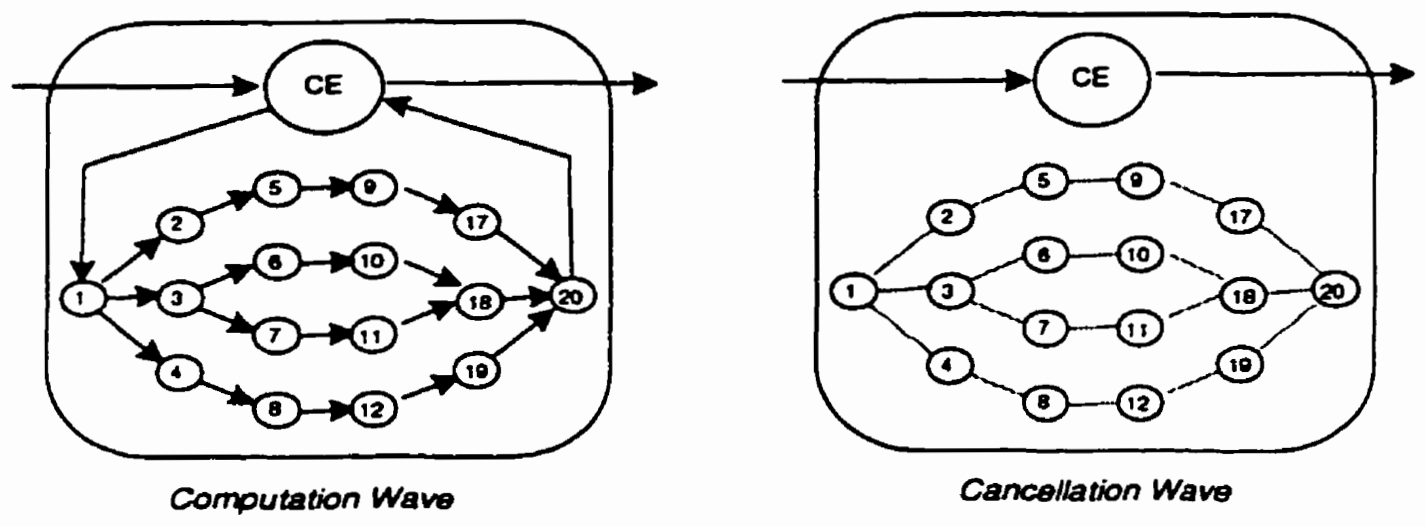

Figure 7.4 : Journey of a computation wave (a) and a cancellation wave (b)

We believe that Clustered Time Warp is more scalable as the size of the simulation model grows. In the following section we compare the performance of Time Warp and Clustered Time Warp on models having different topologies and different sizes.

\subsection{Experiments}

The very small number of large digital circuits publicly available makes it very difficult to study the scalability of asynchronous algorithms. Furthermore, the behavior of a simulation is strongly dependent on the functional characteristics of the circuit, making it difficult to draw general conclusions from a few empirical and possibly atypical results. For these reasons, we chose to use a family of open queueing network models. The advantage of using such models is twofold: first 
it becomes possible to measure directly the effect of size on the performance of the algorithms, second, it allows us to also study the effect of different topologies.

\subsubsection{Environment}

\section{Simulation models}

We used three network models representative of different classes of simulations [Glaz93]: a pipeline model, a hierarchical model and a distributed model.

The pipeline model (figure 7.5 ) is composed of 30 clusters, each of which is composed of $n \times n$ logical processes (see next section). The clusters are organized in nine stages and messages propagate in one direction from two sources to two sinks and are routed randomly at each process.

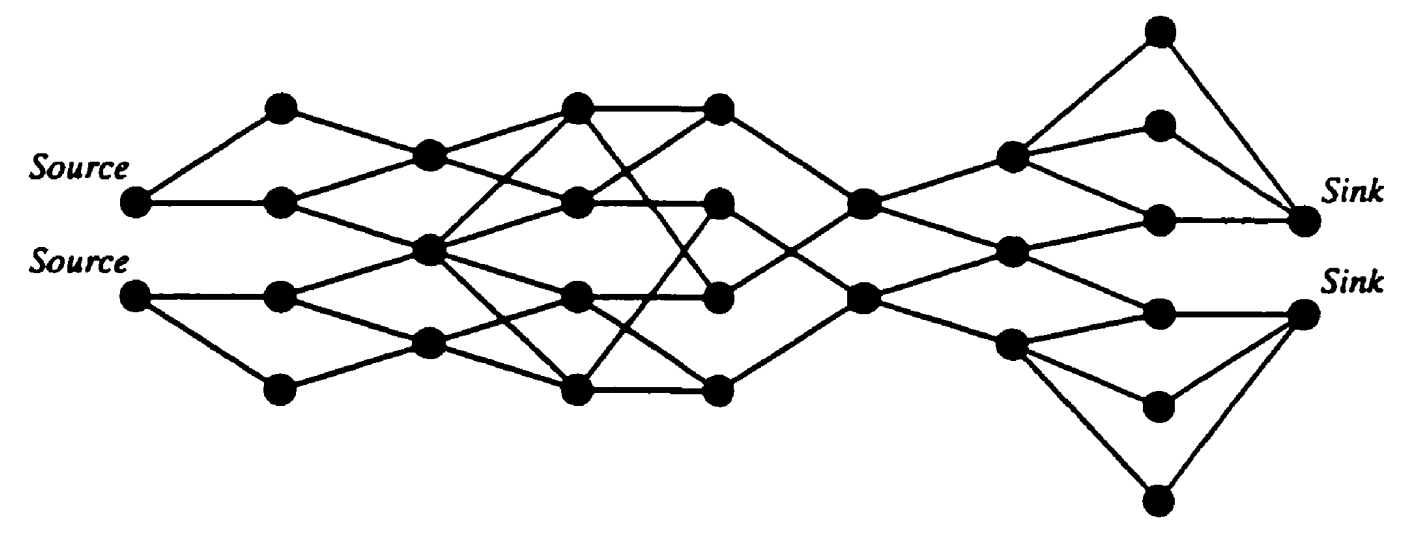

Figure 7.5 : The pipeline model

The hierarchical model (figure $\mathbf{7} .6$ ) has 49 clusters organized in four hierarchical levels. Clusters act as filters, sending fewer messages to neighboring clusters than they receive. The top cluster generates $50 \%$ of the number of messages it receives. Clusters in the middle level generate $33 \%$ of the number of received messages, and clusters located at the bottom of the structure generate $25 \%$ of the received messages. In addition, any cluster, regardless of its level can behave as a source. Messages are routed randomly and can flow in any direction. 


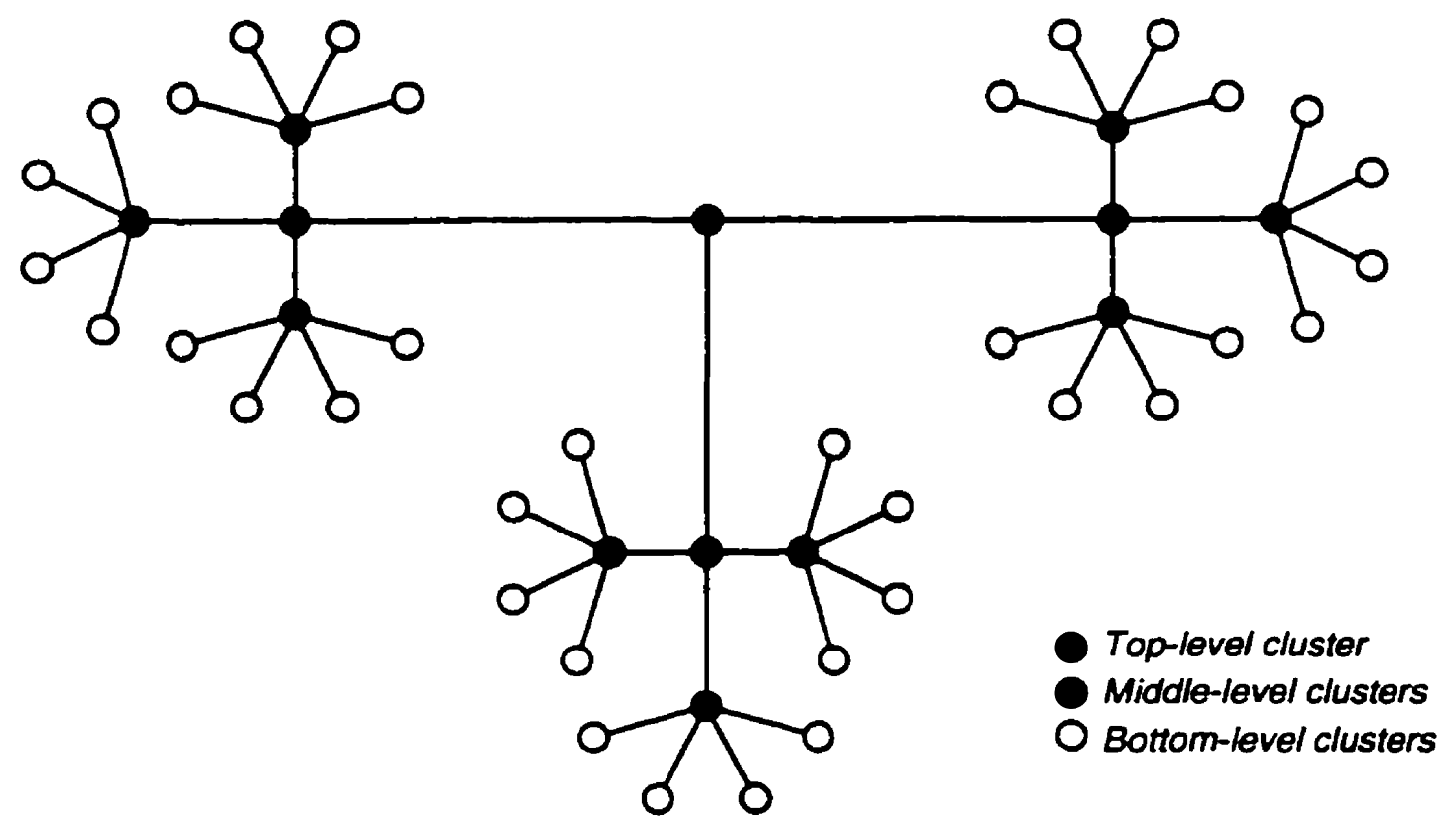

Figure 7.6: The hierarchical network model

The distributed model (figure $\bar{i} . \bar{l}$ ) is composed of 49 clusters organized into five regions. Clusters also act as filters and all of them generate $33 \%$ of the total number of messages they receive. Any cluster can also be a source. Routing is random and all links are bidirectional.

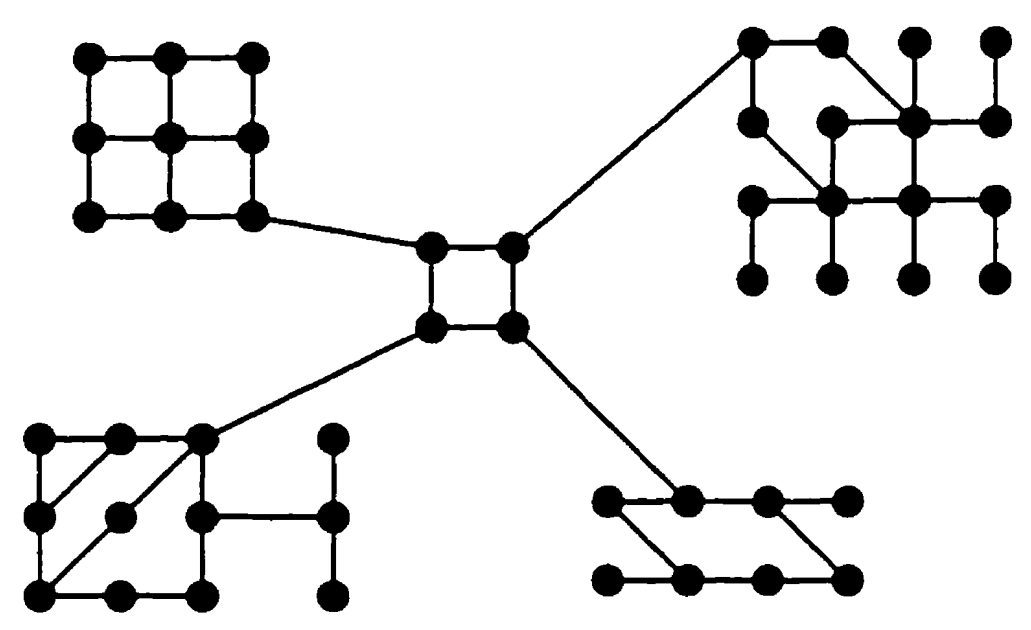

Figure 7.7 : The distributed network model 


\section{Cluster Network}

In all experiments, each cluster is composed of $n \times n$ logical processes connected in a mesh topology. $L P_{i, j}$ denotes the process located at column $i$ and row $j$. All LPs in the first column (ie: $\forall L P_{i, j}$ where $i=1$ ) can receive external messages sent by LPs located in different clusters. Furthermore, LPs in the last column (ie: $\forall L P_{i, j}$ where $i=n$ ) can send messages to other clusters. Figure 7.8 shows an example of a cluster composed of 36 processes. In this example. only processes $(1,1)$ to $(1,6)$ can receive external messages whereas only processes $(6,1)$ to $(6,6)$ can send external messages. All other processes receive and send internal messages only.

It is possible for a cluster to have a size whose square root is not an integer. In this case. the number of elements is reduced in some rows in such a way that the maximum difference in size between any two rows is not greater than one.

If cluster $C_{1}$ has an outgoing link to $C_{2}$, the output of each process in the last column of $C_{1}$ is connected to the process of identical row in the first column of $C_{2}$. For instance. if a cluster size of 36 is used (figure $i .8$ ). then the output of process (6.1) of cluster $C_{1}$ will be connected to the input of process (1.1) of cluster $C_{2}$. Similarly. process $(6,2)$ of $C_{1}$ will be connected to process $(1,2)$ of $C_{2}$, etc. Of course. a cluster can be connected to several other clusters.

A process can forward a message to any of its neighbors, except the one it received the message from. Routing decisions are made randomly at each process.

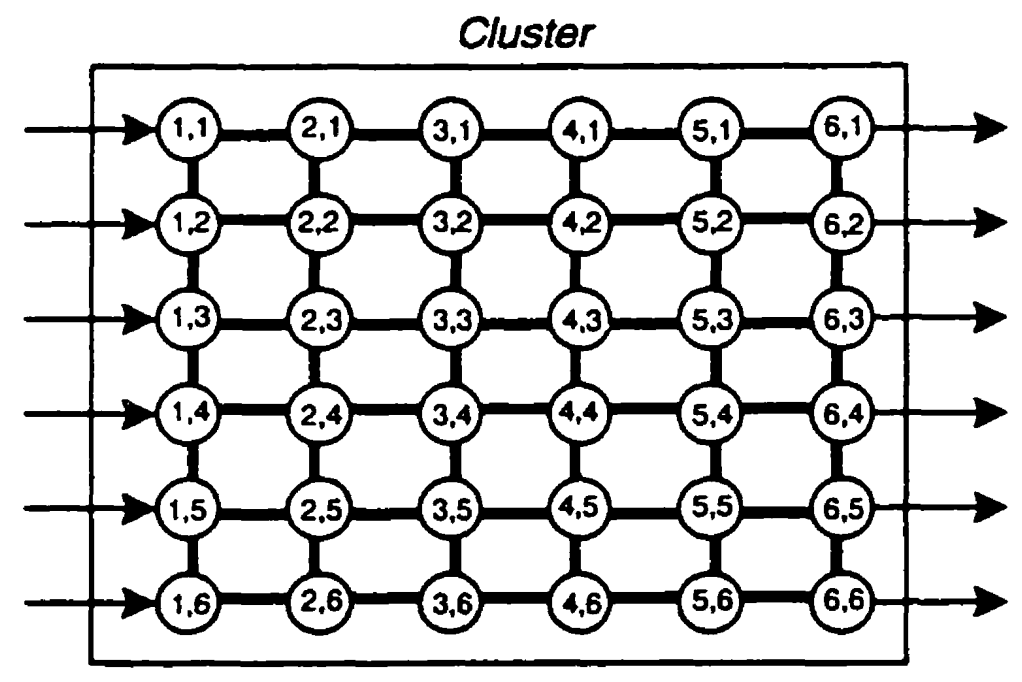

Figure 7.8 : Structure of a cluster 


\section{Service Time Distributions}

Three types of service time distributions were used (table 7.1): a deterministic distribution, a uniform distribution, and a shifted exponential distribution. These distributions are frequently used in the simulation of queueing networks and represents a wide range of models [Gros88, Glaz93, Bouk91].

\begin{tabular}{|c|c|}
\hline Distribution & Random value expression \\
\hline \hline Deterministic & 1.0 \\
\hline Uniform & $0.1+$ uni() \\
\hline Shifted Exponential & $0.1-\log ($ uni()) \\
\hline
\end{tabular}

Table 7.1: Service time distributions

\section{System stimulation}

The events that stimulate the models are generated in a similar way than in a logic simulation. As we have seen previously, part or all of the clusters can act as sources. For instance. the pipeline model has two source clusters whereas all the clusters in the hierarchical and the distributed model are sources. Source clusters are stimulated by a set of input vectors which are generated prior to the beginning of the simulation.

Figure 7.9 depicts the stimulation of the pipeline model. In this example. each source cluster is located at the beginning of the pipeline model (see figure 7.5 ) and each of them contains nine processes connected in a $3 \times 3$ mesh topology. Three input vectors are applied to the first column of processes of each cluster. Because three processes in each cluster can receive external events, a total of six events are generated for each input vector. In total, 18 events are created prior to the beginning of the simulation.

Like in the simulation of a digital circuit (see chapter 1). events in the same input vector have the same timestamp value. In addition, the timestamp difference between each input vector is large enough so that the timestamp of the last event generated in the system and which has been induced by vector $V_{i}$ is smaller than the timestamp of the first event induced by vector $V_{i+1}$. 


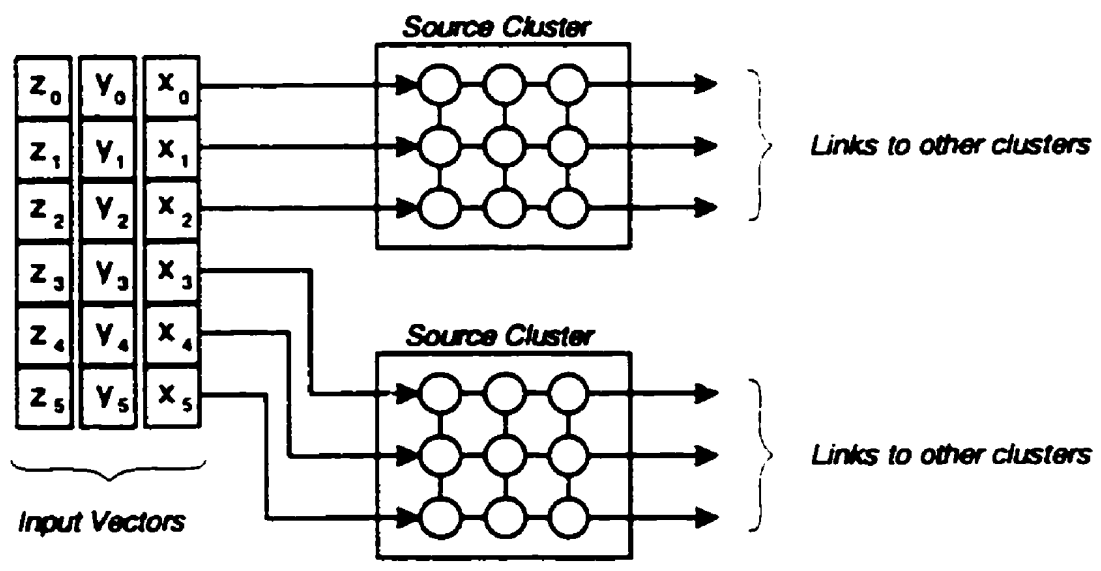

Figure 7.9: Stimulation of the pipeline model

In the experiments described in this chapter, a total of $N$ input vectors $\left\{V_{0}, V_{1} \ldots . . V_{n-1}\right\}$ were generated such that $t_{0}<t_{1}<\ldots<t_{n-1}$, where $t_{i}$ is the timestamp value of the events in input vector $V_{i}$. Events are enqueued into the input queues of the processes in the case of Time Warp, or the input queues of the clusters in the case of Clustered Time Warp. Once the simulation is launched, the system watches the progress of the computation and as soon as the GVT becomes equal or greater than the timestamp value of input vector $V_{1}$, a new input vector $V_{n+1}$ is generated. Similarly, when GVT $\geq t_{2}$, the system generates another input vector $V_{n+2}$. And so on. As a result. $N$ input vectors are always being processed by the simulation system at any time.

The value of $N$ can affect the behavior and the efficiency of the system. If $N$ is too small. the system will not be able to exploit the inherent parallelism available in the simulation model because too few events will be generated. In addition, the underutilization of the system is unlikely to show its weaknesses in terms of scalability. On the other hand, if $N$ is too large, too many input vectors will be generated and will needlessly charge the system. In our experiments. we chose $N$ to be 12 since it represented a good trade-off.

\section{Metrics}

Three metrics were used to characterize the behavior of the simulation: the throughput, the proportion of rolled back events, and the maximum memory usage. The throughput, as defined 
in chapter 5, is the number of effective (or committed) events per second and gives an exact measure of how fast the simulation advances. The higher its value is, the faster the simulation system is. The proportion of rollbacks gives a good indication of how fast the cancellation wave is catching up with the computation wave. This is an important measure to detect potential rollback explosion or unstable behaviors. The maximum memory usage is the maximum amount of memory needed by any processor for the simulation to complete and is an indication of the effectiveness of the fossil collection mechanism.

Each of these metrics is an indicator of the resource requirements needed by a simulation. The throughput relates to Time (speed of the simulation), rollbacks relate to processor utilization. and memory relates to Space.

To evaluate the scalability of both Time Warp and CTW, each of the models described above was simulated with different cluster sizes.

\subsubsection{Results}

Figures 7.10 through 7.18 show the performance results of the simulations performed with the three queueing network models we have described previously. Exact figures can be found in appendix D. Simulations for the pipeline model were ran on 15 processors while 17 processors were used for both the hierarchical and the distributed models.

Figures $7.10, \pi .11$ and $\pi .12$ show the throughput, the proportion of rolled back events and the maximum memory usage respectively for both Time Warp and the Clustered Time Warp algorithm for the pipeline model. We observe that when the size of the simulation model is rather small (up to about 15,000 processes), both algorithms have approximately the same throughput. However. as the size of the model increases, the throughput of Time Warp decreases dramatically. From 3.500 committed events per second for a model size of 9,000 processes, the throughput of Time Warp is nearly halved when the number of processes reaches 36,000 . The throughput of Clustered Time Warp also decreases. but the reduction is less dramatic (about $10 \%$ for a deterministic distribution and about $20 \%$ for an exponential distribution). The cause of the throughput reduction is directly due to the increase in the number of rollbacks. We observe a clear correlation between figure $\bar{i} .10$ and figure 7.11 . For Time Warp. the proportion of rolled 
back events increases dangerously as the size of the simulation grows. For about 9.000 processes, about $11 \%$ of the events are rolled back. The proportion goes up to $35 \%$ for a model size of 36,000 processes. Rollbacks also increase for Clustered Time Warp by about $2 \%$.

The difference in efficiency between the two algorithms is mainly due to the fact that in CTW a cancellation wave travels faster than the computation wave, which is not the case for Time Warp even though antimessages are given higher priority than normal messages. Consequently, as the size of the model grows, wrong computations in Time Warp tend to survive for a longer time, and therefore waste more computational resources than CTW.

The increase in size of the model implies that more events are actually generated for each input vector. This augmentation in the number of events has two side effects. First, more parallelism is available and as a consequence processors have the potential to have a higher throughput. On the other hand, the increase in the number of events also generates a larger overhead for the synchronization algorithm. The simulation system needs more memory to store extra messages and it has to deal with larger event heaps (needed for the scheduling of the processes). This additional overhead is the main reason why the simulation system does not have a higher throughput as the model size grows. Furthermore. the smallest instance of each model utilized most of the computing resources, therefore, the overall throughput of the system could not be improved much. even if an optimal parallel synchronization algorithm is used.

We also observe that in all cases, different service time distributions do not have a pronounced effect on the behavior of the algorithms. However, a deterministic distribution generally performs slightly better than the two other distributions.

There exists a large difference in memory usage between the two algorithms (see figure 7.12 ). For a population of about $2 \pi, 000$ processes. Time Warp memory requirements are 3 times higher than that of Clustered Time Warp. Again, the fact that wrong computation is cancelled much more rapidly in CTW implies that fewer objects are being manipulated by the system. Also. processes do not have individual output queues in Clustered Time Warp. This will also have a positive impact on the fossil collection mechanism. Because the number of fossil objects is likely to decrease, processors waste less time recycling fossil objects.

The hierarchical model (figures $i .13$ to $i .15$ ) exhibits similar behavior than the pipeline model. 
We observe a $30 \%$ reduction for Time Warp when the size of the simulation model grows from 15.000 to 60.000 processes whereas the reduction is less than $10 \%$ for Clustered Time Warp. When compared to the pipeline model, the number of rolled back events in the hierarchical model (figure $i .14$ ) is larger for both algorithms. Still, Time Warp suffers from large overhead due to rollbacks (up to about $30 \%$ ). We also observe that Clustered Time Warp is more sensitive to the size of the hierarchical network model than to the size of the pipeline model. For a network containing about 15,000 processes, $10 \%$ of the events are rolled back with CTW. and the proportion goes up to $15 \%$ when 60,000 processes are used. In the pipeline model, messages were travelling in only one direction. In the hierarchical model. messages can move up and down. therefore clusters are more exposed to rollbacks. In terms of memory usage (figure 7.15 ). the requirements of Clustered Time Warp are half that of Time Warp.

Figures $\bar{T} .16$ to $\bar{i} .18$ show the performance results for the distributed network model. Interestingly, the throughput for Time Warp is much higher than that of Clustered Time Warp when the size of the simulation model is rather small. For about 15.000 processes. Time Warp and CTW have a throughput of about 4.500 and 3.500 events per second respectively. Similarly, the proportion of rollbacks is smaller for Time Warp. About $10 \%$ of the events are rolled back whereas nearly $20 \%$ of events are rolled back for Clustered Time Warp. The distributed nature of the model is responsible for the overhead incurred on the CTW algorithm. When the model contains about $3 \pi, 000$ processes, both algorithms have approximately the same throughput (about 3.000 events per second). but when the simulation reaches 60.000 processes. Time Warp becomes inefficient. more than $40 \%$ of its events are being rolled back and its throughput has decreased by $60 \%$. From 15.000 to 60.000 processes, the throughput of Clustered Time Warp has decreased by less then $15 \%$. Once again, the memory requirements of Clustered Time Warp are about half that of Time Warp and in both cases, they stay at a constant level.

\subsection{Summary}

In this chapter, we have shown that optimistic algorithms may seriously suffer from rollbacks getting out of control, especially in large simulation models of several thousands of processes. Scalability therefore becomes an important issue. 
We suggest that Clustered Time Warp may have the capability to contain (to a certain extent), the explosion of rollbacks found in Time Warp. The degradation of performance due to phenomena such as cascading rollbacks and the dog chasing its tail could therefore be limited.

Using three models of different sizes and service time distributions, we demonstrated that in all of the cases studied, Clustered Time Warp is more scalable than Time Warp. Generally, the throughput of Time Warp decreases from 2 to 5 times faster than Clustered Time Warp.

Finally, we have also shown that the memory requirements for CTW are 3 times smaller than that of Time Warp for the pipeline model, and twice smaller for the two other models. 


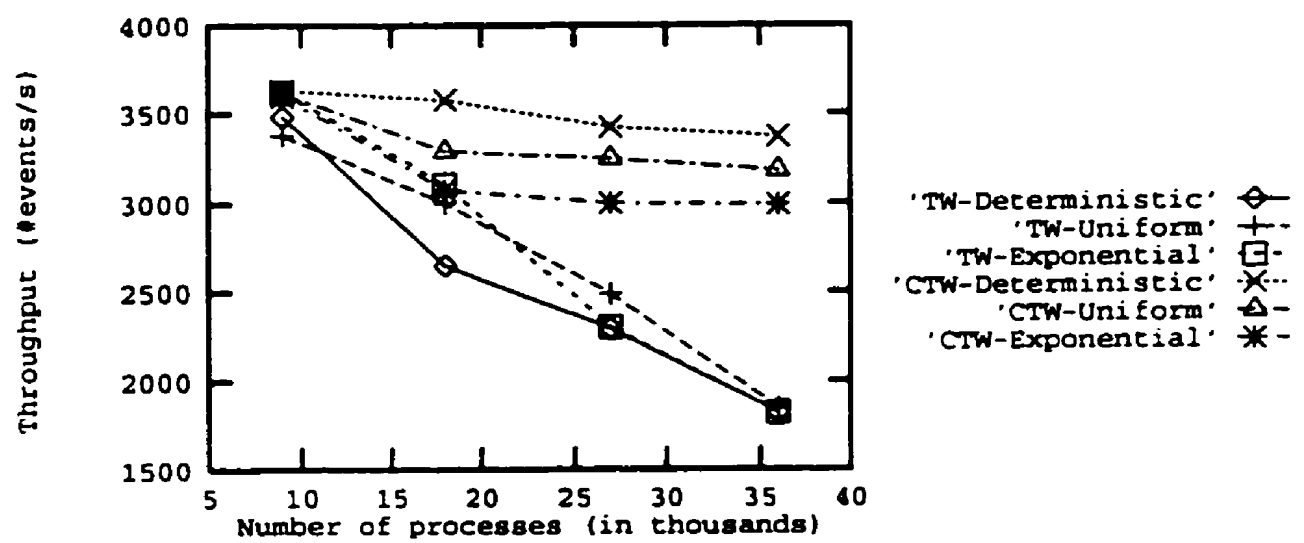

Figure 7.10 : Throughput for the pipeline model

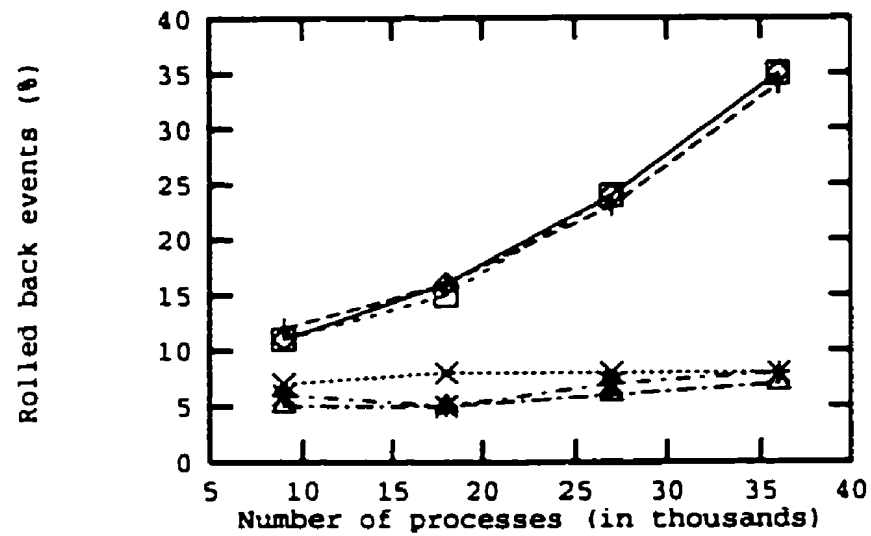

'TW-Deterministic' $\theta$ 'Tw-Uni form. + TW-Exponential. 'CTW-Deterministic' 'X.. CTW-Uni form' $\Delta-$ 'CTW-Exponential. $*$ -

Figure 7.11 : Rollbacks for the pipeline model

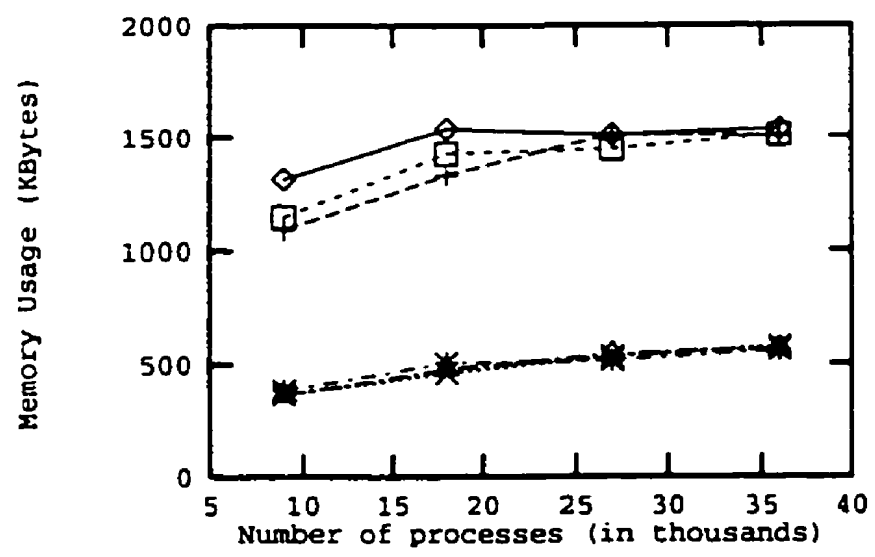

'TW-Deterministic' $\theta$ 'Tw-Uniform' +TW-Exponential' $\exists$ 'CTW-Deterministic' - $\times$.. 'CTW-Uniform' ACTW-Exponential. * -

Figure 7.12: Memory usage for the pipeline model 

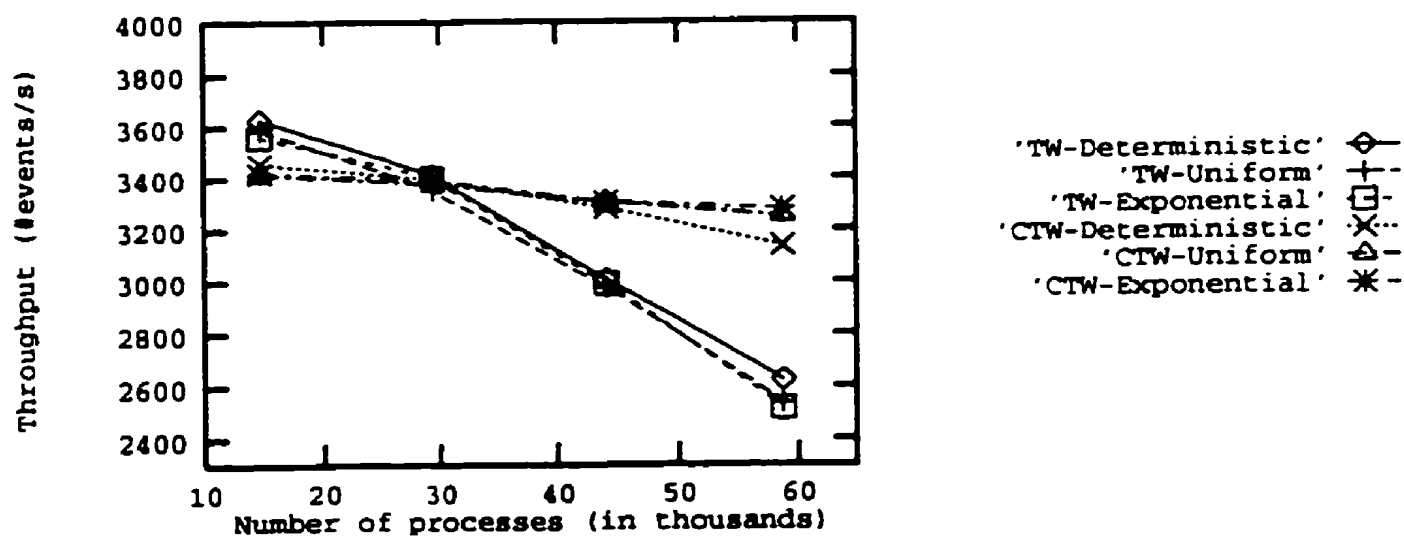

Figure 7.13 : Throughput for the hierarchical model
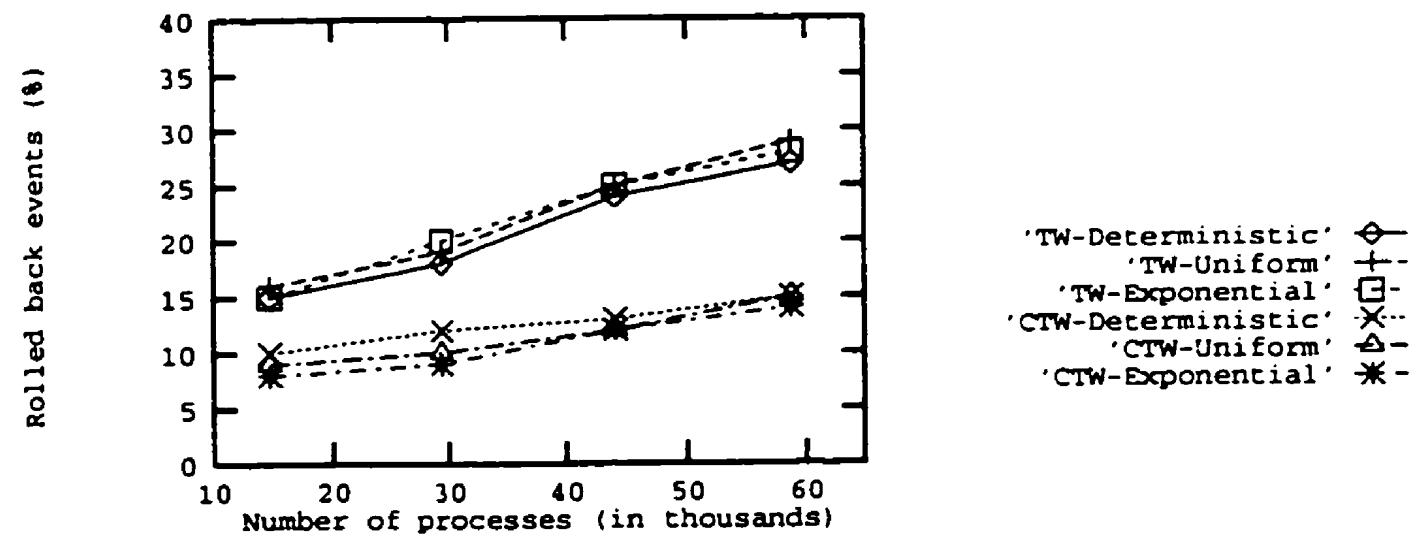

Figure 7.14 : Rollbacks for the hierarchical model
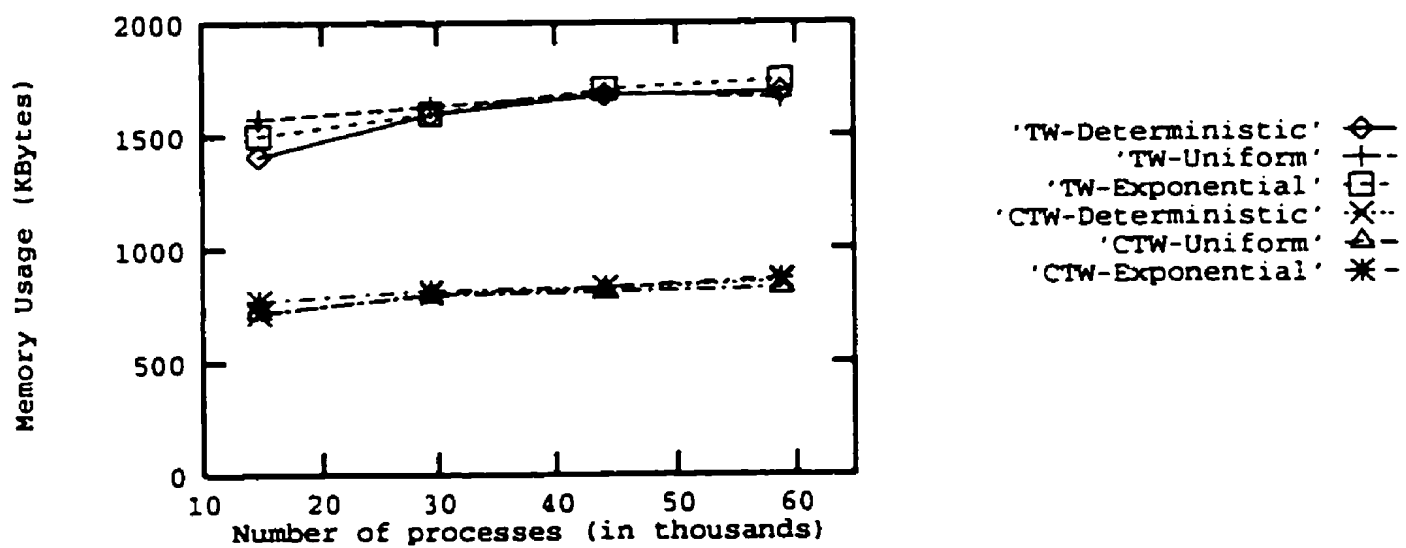

Figure 7.15: Memory usage for the hierarchical model 

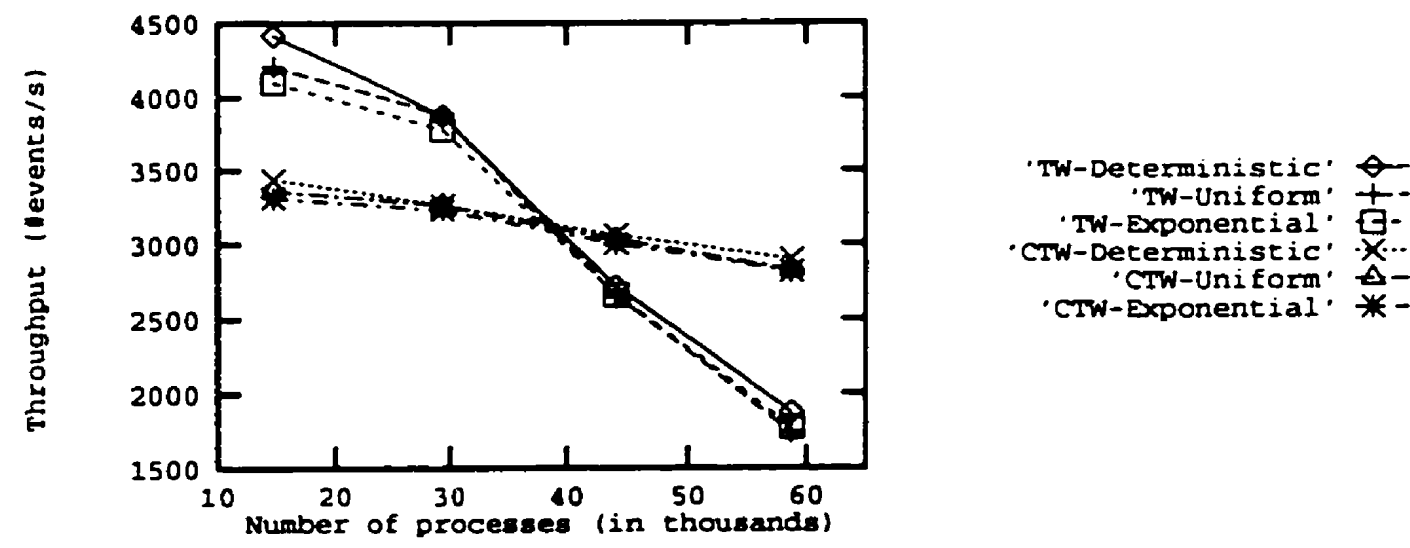

Figure 7.16 : Throughput for the distributed model
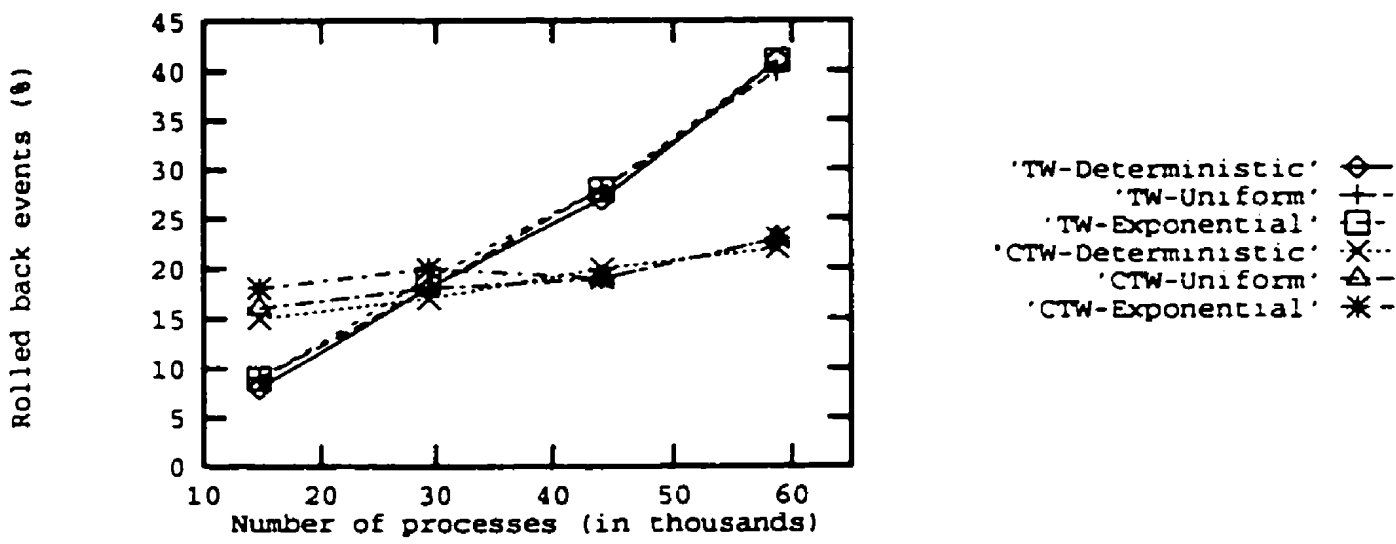

Figure 7.17 : Rollbacks for the distributed model
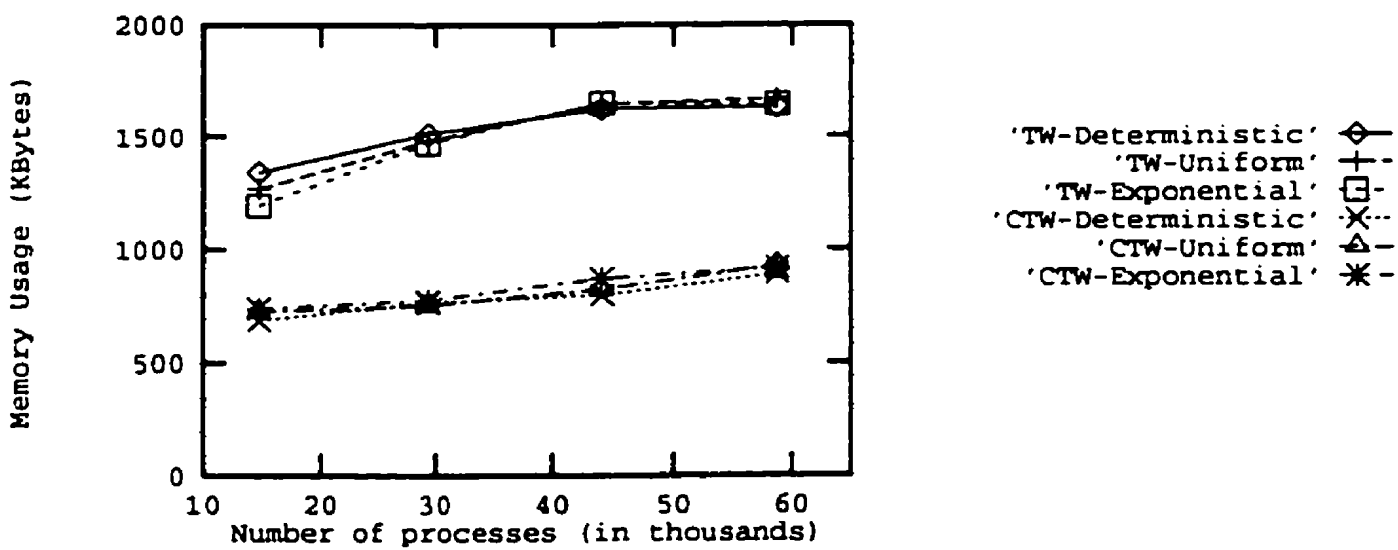

Figure 7.18: Memory usage for the distributed model 


\section{Chapter 8}

\section{Conclusion}

The increasing size and complexity of VLSI circuits make the use of software parallel simulators a very attractive alternative to hardware simulators. It is recognized that asynchronous algorithms can extract more parallelism than synchronous algorithms such as time-stepped techniques. However. the synchronization overhead is critical to the success of asynchronous approaches.

Conservative techniques (blocking) suffer from the low-computational granularity exhibited by logic circuit models which can overwhelm the simulation system with deadlocks. On the other hand. optimistic techniques (non-blocking) are likely to be prone to rollback explosions and an excessive usage of the memory.

In this thesis. we are interested in the design of an asynchronous approach to parallel discreteevent simulation targeted at the simulation of large digital circuits which addresses three major issues: efficiency, memory usage, and scalability.

In this chapter. we summarize our contributions to the field of parallel logic simulation and outline possible directions for future research.

\subsection{Contributions}

The contributions of this thesis to the field of parallel discrete-event simulations of logic-level circuits are as follows:

- We have analyzed the characteristics of digital circuits and have shown that asynchronous algorithms can potentially achieve speedups 3 to 15 times higher than synchronous algorithms such as time-stepped techniques. 
We have also shown that the workload is not necessarily evenly distributed among the gates of the circuit. In the circuits studied, less than $20 \%$ of the gates are responsible for most of the circuit activity. Furthermore, we have observed that the variability of the workload for the circuit is relatively small. For all circuits which we examined. we have shown that the variation of the workload of each gate measured at the end of the presimulation (which represented $10 \%$ of the whole simulation) staved within a range of $10 \%$ and less. This results suggest that pre-simulation can be used to extract load information and to dynamically balance the simulation.

Finally, we have pointed out the presence of a very large number of cycles in the circuits.

- We have introduced Clustered Time Warp, a novel hybrid algorithm for the parallel simulation of discrete event models which takes advantage of the characteristics of logic simulation and which can be implemented on general purpose distributed memory architecture. This algorithm was developed with three main concerns in mind:

- Efficiency: The algorithm had to give good results with regard to the simulation time.

- Low-cost Memory: The memory requirements of the algorithm had to be less than other optimistic techniques such as Time Warp (with or without Periodic State Saving).

- Scalability: The algorithm had to be scalable and less sensitive to phenomena such as rollback explosions.

- We have also presented a family of three space-based checkpointing algorithms:

- Clustered Rollback. Clustered Checkpoint (CRCC).

- Local Rollback, Clustered Checkpoint (LRCC).

- Local Rollback. Local Checkpoint (LRLC).

Each of these techniques occupies a different point in the spectrum of possible trade-offs between memory usage and execution time. 
When compared to pure Time Warp, Clustered Time Warp with CRCC can save more than $40 \%$ of the memory usage on the average, whereas LRCC and CRCC save 22 and $15 \%$ of the memory usage respectively. As to the simulation time, CRCC is slower than Time Warp, whereas the two other algorithms exhibited a speed comparable to Time Warp.

Results have also shown the importance of taking into consideration the memory required to store events, especially when the event size is comparable to that of a process state.

- We have introduced a dynamic load balancing algorithm for Clustered Time Warp. We have shown that the clustering approach reduces considerably the amount of information to be manipulated by a dynamic load balancing algorithm. We have also described a triggering technique based on the throughput of the simulation system. Results also show that improvements from 40 to $100 \%$ can be achieved.

- We have analyzed the effect of partitioning and mapping on the behavior and the performance of the simulation system. We showed that a partitioning algorithm which tries to minimize the number of cutsets between the partitions does not necessarily succeed in minimizing inter-processor communications. Moreover, we showed that in our environment. load imbalance had a stronger effect than rollback overhead. For the system to achieve a better performance, the analysis showed that a dynamic algorithm is actually needed to find a partitioning and a mapping of the problem on the target architecture.

- Finally, we have investigated the scalability of Clustered Time Warp with the CRCC checkpointing techniques using different queueing network models. We have shown that. when compared to Time Warp, the algorithm behaves rather well as the size of the model grows. Even though the performance of CTW is not as good as that of Time Warp for small graphs, we have shown that CTW outperforms Time Warp for large models. Furthermore, we have shown that the memory requirements for CTW are 3 times smaller than that of Time Warp for the pipeline model, and half as large for the two other models [Glaz93]. 


\subsection{Future Work}

We can identify several directions for future research:

- The Clustered Time Warp algorithm could be extended to dynamically partition the simulated model into clusters. Using a heuristic algorithm along with pre-simulation. CTW could decide on a good cluster partition on the fly that would minimize memory usage and maximize efficiency.

- Because of the difficulty to obtain the netlist or the VHDL description of digital circuits from manufacturers. this research was limited to the use of the ISCAS 89 benchmark series. Even though these circuits are widely accepted by the research community, the largest circuit available in this series contains about 20,000 gates. which is relatively small with respect to the size of today's VLSI chips. Hence, there is a need to validate the results presented in this thesis with much larger circuits whose sizes are several orders of magnitude larger than that of the ISCAS'89 circuits.

- In this thesis. random input vectors were used to stimulate the circuits. However. this might not reflect reality and real input vectors could activate different parts of the simulated circuits. Therefore, in addition to larger circuits. there is a need to use a set of real input vectors to place the simulation system in real conditions. The use of real input vectors could highlight other characteristics of digital circuits and the study of different partitioning and mapping strategies could be extended.

- It would be interesting to port the simulator onto a distributed memory architecture such as the Hypercube.

- Porting the system onto a network of workstations would also be interesting. The message latency of local area networks could then play a major role in the efficiency of the algorithm.

- The current implementation of the Clustered Time Warp simulator can only use netlist descriptions of the ISCAS 89 benchmark circuits. Making the system capable of reading VHDL descriptions would definitely widen the range of circuits which can be used. 
- It would be interesting to compare the performance of Clustered Time Warp with conservative techniques such as the null-message [Chan79] or the SGTNE [Bouk95] approaches. 


\section{Appendix A}

\section{Parallelism available in the ISCAS'89 Benchmark Circuits}

Average number of concurrent events

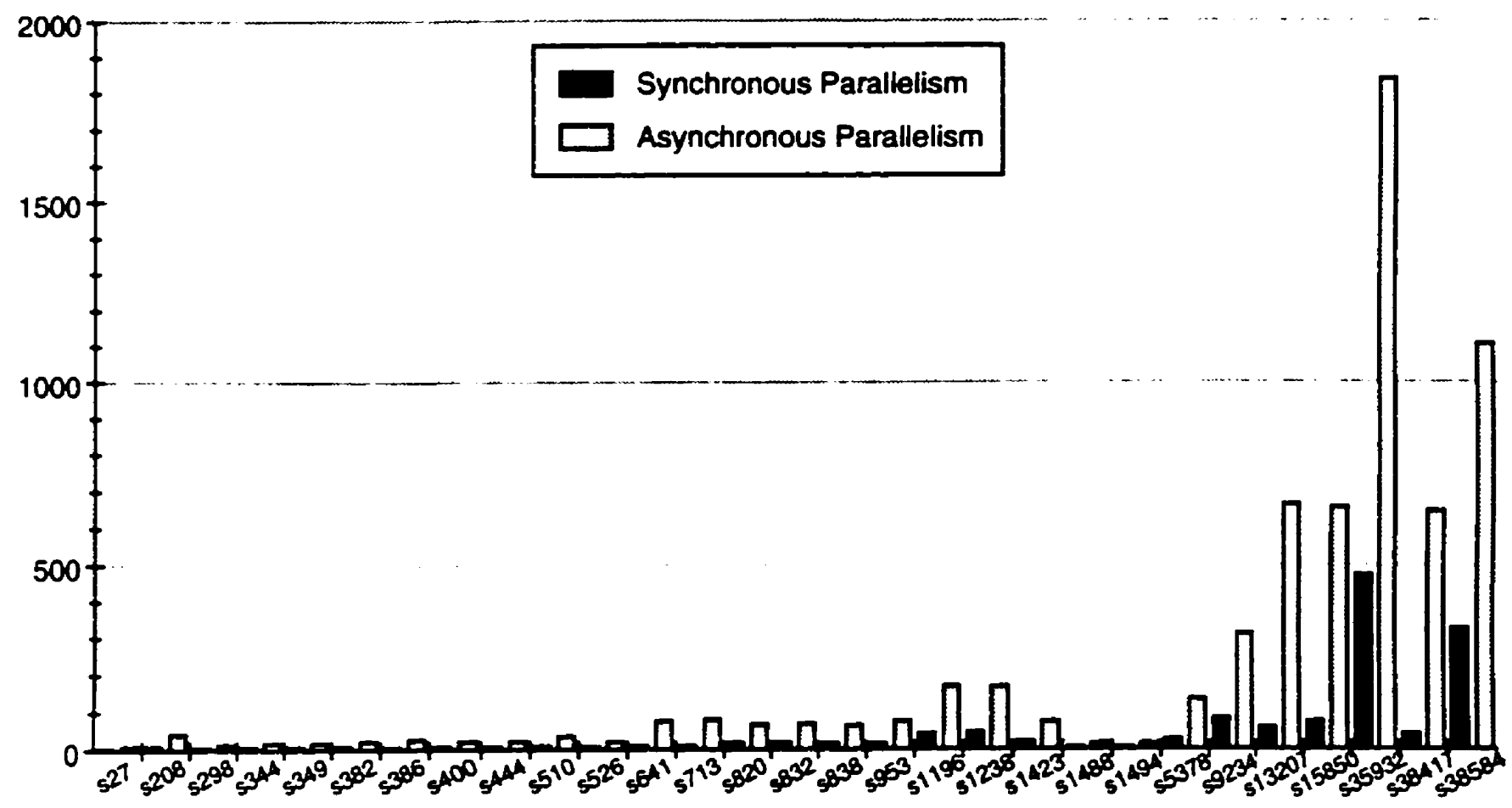

Circuits

Figure A.1: Average parallelism measured in the ISCAS'89 Benchmark circuits 
Figure A.1 shows both the synchronous and the asynchronous parallelism measured for each circuit of the ISCAS'89 benchmark suite [Brgl89]. 100 random test vectors were applied to circuits s15850, s35932, s38417 and s38584. For the other circuits. 200 test vectors were applied. The results clearly show that in all cases, asynchronous algorithms can extract more parallelism than synchronous algorithms. Detailed results are given in table A.1. These results are discussed in chapter 2.

\begin{tabular}{|c|c|c|c|c|c|c|c|}
\hline \multirow[t]{2}{*}{ Circuit } & \multirow[t]{2}{*}{ Size } & \multirow[t]{2}{*}{ Inputs } & \multirow[t]{2}{*}{ Outputs } & \multicolumn{2}{|c|}{ Synchronous Parallelism } & \multicolumn{2}{|c|}{ Asynchronous Parallelism } \\
\hline & & & & Average & Relative (\%) & Average & Relative (\%) \\
\hline $\mathbf{s} 27$ & 18 & 4 & 1 & 3.2 & 17.0 & 10 & 55.6 \\
\hline s208 & 117 & 11 & 2 & 10 & $\overline{8.54}$ & 42 & $\overline{35.9}$ \\
\hline s298 & 142 & 3 & 6 & 5.3 & 3.73 & 14 & 9.86 \\
\hline s344 & 195 & $\overline{9}$ & 11 & 8.2 & 4.21 & $\overline{19}$ & 9.14 \\
\hline$\overline{s 349}$ & 196 & 9 & 11 & $\overline{8.2}$ & 4.18 & 19 & 9.69 \\
\hline $\mathbf{s 3 8 2}$ & 188 & 3 & 6 & 8 & 4.26 & 23 & 12.2 \\
\hline s386 & 179 & 7 & $\bar{i}$ & 7.6 & 4.25 & 28 & 15.6 \\
\hline 5400 & $\overline{192}$ & $\overline{3}$ & 6 & 8.1 & 4.22 & 23 & 12.0 \\
\hline 5444 & $\overline{211}$ & 3 & 6 & 8.3 & $\overline{3.93}$ & 23 & 10.9 \\
\hline 5510 & 243 & 19 & $\vec{\imath}$ & 10 & 4.12 & 36 & 14.8 \\
\hline 5526 & $\overline{224}$ & 3 & 6 & 7.9 & 3.53 & 21 & 9.37 \\
\hline s641 & $45 \bar{t}$ & 35 & 24 & $\overline{9.7}$ & $\overline{2.12}$ & $\overline{i T}$ & 16.8 \\
\hline $\mathbf{s i 1 3}$ & 470 & 35 & 23 & 10 & 2.12 & $\overline{81}$ & 17.2 \\
\hline 5820 & 331 & 18 & 19 & 20 & 6.04 & 69 & 20.8 \\
\hline s832 & 329 & 18 & 19 & 20 & 6.08 & 70 & 21.3 \\
\hline 5838 & 459 & 35 & 2 & 19 & 4.14 & 66 & 14.4 \\
\hline s953 & 463 & 16 & 23 & 17 & 3.67 & $\pi$ & 16.6 \\
\hline s1196 & 575 & 14 & 14 & 44 & 7.65 & 172 & 29.9 \\
\hline s1238 & 554 & 14 & 14 & 46 & 8.30 & 171 & $\overline{30.9}$ \\
\hline s1423 & 753 & 17 & 5 & 21 & 2.79 & 76 & 10.1 \\
\hline s1488 & 686 & 8 & 19 & 5.8 & 0.85 & 16 & 2.33 \\
\hline s1494 & 680 & 8 & 19 & 5.7 & 8.38 & 16 & 2.35 \\
\hline 55378 & 3,042 & 35 & 49 & 27 & 0.89 & 136 & 4.47 \\
\hline s9234 & 5.866 & 19 & 22 & 84 & 1.43 & 314 & 5.35 \\
\hline s1320t & 8.772 & 31 & 121 & 59 & $\overline{0.67}$ & 666 & 7.59 \\
\hline s15850 & 10.470 & 14 & 87 & 76 & 0.72 & 658 & 6.28 \\
\hline s35932 & 18,148 & 35 & 320 & 474 & 2.61 & 1,839 & 10.1 \\
\hline s38417 & 23,949 & 28 & 106 & 43 & 0.17 & 650 & 2.71 \\
\hline s38584 & 20,995 & 12 & 278 & $3 \overline{28}$ & 1.56 & $1,10 \overline{7}$ & 5.27 \\
\hline
\end{tabular}

Table A.1: Parallelism measured in the ISCAS'89 Benchmark circuits 


\section{Appendix B}

\section{Quantitative Results of Logic Simulations}

These tables present the performance results of the simulation of two of the largest circuits of the ISCAS' 89 benchmark suite, for both the Time Warp and the Clustered Time Warp algorithms.

Tables B.1 and B.2 show the results for circuits s35932 and s38584 respectively with different cluster sizes and for each checkpointing strategies of Clustered Time Warp. For each simulation. the simulation time and the peak memory usage were measured. The simulation time is the total time (in seconds) needed by the system to complete the simulation. The peak memory usage is the maximum memory (in Kbytes) needed by any processor to complete the simulation. Note that since Time Warp does not have clusters, its simulation time and memory usage are both constant with respect to the cluster size. All of the simulations whose results are presented in tables B.1 and B. 2 were ran on 20 processors of the BBN Butterfly.

Tables B.3 to B. 8 show the performance results for circuits s35932 and s3858t respectively. For each checkpointing strategy of Clustered Time Warp, the best cluster size was chosen based on the previous experiments. One cluster per processor was used for both LRLC and LRCC. and for CRCC, 32 and 128 clusters per processors were used for circuits s35932 and s38584 respectively. Then, the same simulation was ran on a different number of processors and different measurements were made. In addition to the simulation time and the peak memory usage, the peak number of states stored by any processor was also measured.

Finally, tables B.9 and B.10 give the speedup obtained when using the different parallel simulation algorithm. 


\begin{tabular}{|c|c|c|c|c|c|c|}
\hline \multicolumn{7}{|c|}{ Circuit s35932 } \\
\hline \multirow{2}{*}{$\begin{array}{c}\text { Number of } \\
\text { clusters } \\
\text { per } \\
\text { processors }\end{array}$} & \multicolumn{3}{|c|}{$\begin{array}{c}\text { Simulation Time } \\
\text { (seconds) } \\
\mathrm{TW}=35 \mathrm{sec} .\end{array}$} & \multicolumn{3}{|c|}{$\begin{array}{c}\text { Peak Memory Usage } \\
\text { (Kbytes) } \\
T W=495 \text { KBytes }\end{array}$} \\
\hline & $\mathrm{CRCC}$ & $\overline{\mathrm{LRCC}}$ & LRLC & $\overline{\mathrm{CRCC}}$ & $\overline{\text { LRCC }}$ & LRLC \\
\hline 1 & 117 & $\overline{38}$ & 32 & 612 & 332 & 396 \\
\hline 2 & 75 & 38 & 30 & 343 & 302 & 382 \\
\hline 4 & 71 & 38 & 31 & 309 & 315 & 383 \\
\hline 8 & 66 & 40 & 31 & 309 & 352 & 415 \\
\hline 16 & 62 & 40 & 30 & 304 & 344 & 412 \\
\hline 32 & 52 & 41 & 30 & 301 & 348 & 406 \\
\hline 64 & 48 & 42 & 30 & 300 & 350 & 404 \\
\hline 128 & 47 & 42 & 31 & 301 & 353 & 403 \\
\hline 256 & 47 & 44 & 31 & 300 & 351 & 403 \\
\hline
\end{tabular}

Table B.1: Effects of cluster size on circuit s35932

\begin{tabular}{|c|c|c|c|c|c|c|}
\hline \multicolumn{7}{|c|}{ Circuit s38584 } \\
\hline \multirow{2}{*}{$\begin{array}{l}\text { Number of } \\
\text { clusters } \\
\text { per } \\
\text { processors }\end{array}$} & \multicolumn{3}{|c|}{$\begin{array}{c}\text { Simulation Time } \\
\text { (seconds) } \\
\text { TW }=40 \mathrm{sec}\end{array}$} & \multicolumn{3}{|c|}{$\begin{array}{c}\text { Peak Memory Usage } \\
\text { (Kbytes) } \\
\mathrm{TW}=30 \tau \mathrm{KBytes}\end{array}$} \\
\hline & CRCC & LRCC & LRLC & $\overline{C R C C}$ & LRCC & LRLC \\
\hline 1 & 109 & 47 & 41 & 498 & 220 & 292 \\
\hline 2 & 97 & 45 & 40 & 526 & 296 & 290 \\
\hline 4 & 92 & 45 & 39 & 552 & 407 & 290 \\
\hline 8 & 90 & 45 & 38 & 311 & 307 & 288 \\
\hline 16 & 83 & 44 & 38 & $28 T$ & 291 & 288 \\
\hline 32 & 60 & 44 & 38 & 216 & 263 & 285 \\
\hline 64 & 51 & 45 & 38 & 214 & 286 & $2 \pi$ \\
\hline 128 & 50 & 46 & 38 & 173 & 260 & 310 \\
\hline 256 & 51 & 45 & 38 & 222 & 302 & 382 \\
\hline
\end{tabular}

Table B.2: Effects of cluster size on circuit $\mathbf{s} 38584$ 


\begin{tabular}{|c||c|c|c|c|c|}
\hline \multicolumn{6}{|c|}{ Circuit s35932 - Peak number of states } \\
\hline \# procs & CRCC & LRCC & LRLC & PSS & TW \\
\hline \hline 8 & 3102 & 5204 & 2702 & 4610 & 6851 \\
12 & 2853 & 4298 & 1905 & 3812 & 5568 \\
16 & 3404 & 3668 & 1754 & 3035 & 4714 \\
20 & 3052 & 3186 & 1308 & 3680 & 5205 \\
24 & 3058 & 3621 & 2211 & 4061 & 5732 \\
\hline
\end{tabular}

Table B.3: Number of processors vs peak number of states for circuit s35932

\begin{tabular}{|c||c|c|c|c|c|}
\hline \multicolumn{6}{|c|}{ Circuit s35932 - Peak memory usage (Kbytes) } \\
\hline \# procs & CRCC & LRCC & LRLC & PSS & TW \\
\hline \hline 8 & 326 & 571 & 516 & 552 & 651 \\
12 & 296 & 416 & 432 & 461 & 508 \\
16 & 309 & 343 & 425 & 408 & 463 \\
20 & 307 & 335 & 401 & 422 & 492 \\
24 & 310 & 380 & 405 & 463 & 550 \\
\hline
\end{tabular}

Table B.4: Number of processors vs memory for circuit s35932

\begin{tabular}{|c||c|c|c|c|c|}
\hline \multicolumn{6}{|c|}{ Circuit s35932 - Simulation Time (seconds) } \\
\hline \# procs & CRCC & LRCC & LRLC & PSS & TW \\
\hline \hline 8 & 138 & 81 & 73 & 80 & 77 \\
12 & 85 & 60 & 48 & 52 & 50 \\
16 & 65 & 49 & 40 & 44 & 42 \\
20 & 53 & 38 & 33 & 36 & 35 \\
24 & 42 & 35 & 30 & 31 & 32 \\
\hline
\end{tabular}

Table B.5: Number of processors vs simulation time for circuit s35932 


\begin{tabular}{|c||c|c|c|c|c|}
\hline \multicolumn{6}{|c|}{ Circuit s38584 - Peak number of states } \\
\hline \# procs & CRCC & LRCC & LRLC & PSS & TW \\
\hline \hline 8 & 2246 & 2241 & 2252 & 2612 & 3608 \\
12 & 2361 & 2087 & 1519 & 1760 & 2748 \\
16 & 2412 & 1961 & 1306 & 1822 & 2915 \\
20 & 1912 & 2104 & 1216 & 2034 & 3101 \\
24 & 2006 & 2258 & 1194 & 2153 & 3407 \\
\hline
\end{tabular}

Table B.6: Number of processors vs peak number of states for circuit 338584

\begin{tabular}{|c||c|c|c|c|c|}
\hline \multicolumn{6}{|c|}{ Circuit s38584 - Peak memory usage (Kbytes) } \\
\hline \# procs & CRCC & LRCC & LRLC & PSS & TWi \\
\hline \hline 8 & 192 & 235 & 253 & 290 & 326 \\
12 & 221 & 216 & 255 & $23 i$ & 271 \\
16 & 261 & 195 & 265 & 272 & 301 \\
20 & 182 & 206 & 286 & 274 & $30 i$ \\
24 & 194 & 210 & 295 & 272 & 313 \\
\hline
\end{tabular}

Table B. $\bar{i}$ : Number of processors vs memory for circuit s38584

\begin{tabular}{|c||c|c|c|c|c|}
\hline \multicolumn{6}{|c|}{ Circuit s38584 - Simulation Time (seconds) } \\
\hline \# procs & CRCC & LRCC & LRLC & PSS & TW \\
\hline \hline 8 & 75 & 67 & 59 & 64 & 60 \\
12 & 62 & 57 & 50 & 54 & 52 \\
16 & 56 & 50 & 42 & 46 & 44 \\
20 & 51 & 46 & 40 & 42 & 41 \\
24 & 50 & 45 & 39 & 42 & 40 \\
\hline
\end{tabular}

Table B.8: Number of processors vs simulation time for circuit s38584 


\begin{tabular}{|c||c|c|c|c|c|}
\hline \multicolumn{6}{|c|}{ Circuit s35932 - Speedup } \\
\hline \# procs & CRCC & LRCC & LRLC & PSS & TW \\
\hline \hline 8 & 2.05 & 3.49 & 3.88 & 3.53 & 3.67 \\
12 & 3.33 & 4.72 & 5.90 & 5.44 & 5.66 \\
16 & 4.35 & 5.78 & 7.07 & 6.43 & 6.74 \\
20 & 5.34 & 7.45 & 8.57 & 7.86 & 8.08 \\
24 & 6.74 & 8.09 & 9.43 & 9.13 & 8.84 \\
\hline
\end{tabular}

Table B.9: Speedup for circuit s35932

\begin{tabular}{|c||c|c|c|c|c|}
\hline \multicolumn{6}{|c|}{ Circuit s38584 - Speedup } \\
\hline \# procs & CRCC & LRCC & LRLC & PSS & TW \\
\hline \hline 8 & 3.88 & 4.34 & 4.93 & 4.55 & 4.85 \\
12 & 4.69 & 5.11 & 5.82 & 5.39 & 5.60 \\
16 & 5.19 & 5.82 & 6.93 & 6.33 & 6.61 \\
20 & 5.71 & 6.32 & 7.27 & 6.93 & 7.10 \\
24 & 5.82 & 6.47 & 7.46 & 6.93 & $7.2 i$ \\
\hline
\end{tabular}

Table B.10: Speedup for circuit s38584 


\section{Appendix C}

\section{Quantitative Results of Partitioning Algorithms}

Table C.1 shows in percent the relative size of the cutset for four partitioning algorithms applied to the four largest ISCAS' 89 benchmark circuits.

The net size is the total number of gate connections in the circuit. For different sizes of partitions. the cutset size was calculated. Figures in the table show the size of the cutset relative to the net size of the circuit. For instance. circuit s38584 contains 35.938 nets. and when a random partitioning algorithm is used to get two partitions, we obtained a total number of 17.358 nets which were crossing the two partitions. Therefore, $35,938 / 17,35 i=48.3 \%$ represents the relative size of the cutset.

Results were obtained when using a random partitioning algorithm. a string partitioning algorithm, and an input cone and output cone partitioning algorithm.

Discussion of the results are in chapter 6. 


\begin{tabular}{|c|c|c|c|c|c|c|c|c|c|}
\hline \multirow[b]{2}{*}{ Circuit } & \multirow{2}{*}{$\begin{array}{l}\text { Partitioning } \\
\text { Algorithm }\end{array}$} & \multicolumn{8}{|c|}{ \# of partitions } \\
\hline & & 2 & 4 & 8 & 16 & 32 & 64 & 128 & 256 \\
\hline \multirow{3}{*}{ s38584 } & Random & 48.3 & 73.1 & 85.7 & 92.3 & 95.8 & 97.6 & 98.6 & 99.1 \\
\hline & String & 22.1 & 51.0 & 69.3 & 78.8 & 86.8 & 89.6 & 91.2 & 92.7 \\
\hline & Input Cone & 22.0 & 33.2 & 39.6 & 43.6 & $3 \overline{7} .0$ & 35.1 & 36.0 & 39.2 \\
\hline \multirow{3}{*}{$\begin{array}{l}35.938 \text { nets } \\
1.452 \text { flipflops }\end{array}$} & Sensitive Input Cone & 20.7 & 21.5 & 25.9 & 25.1 & 29.8 & 31.8 & 35.3 & 38.6 \\
\hline & Output Cone & 11.8 & 18.4 & 23.7 & 24.5 & 28.5 & 31.4 & 35.3 & 39.0 \\
\hline & Sensitive Ou & 6.39 & 9.83 & 13.0 & 14.6 & 16.3 & 20.8 & 26.2 & 33.3 \\
\hline \multirow{3}{*}{ s38417 } & Random & 47.9 & $\overline{72 . \bar{T}}$ & $\overline{85 . i}$ & $\overline{92.2}$ & $\overline{95 . \bar{i}}$ & $\overline{9 \bar{\imath} .6}$ & $\overline{98.5}$ & 99.1 \\
\hline & StriI & 23.0 & 46.6 & 77.3 & 84.7 & 88.3 & $90 . \bar{\imath}$ & 92.4 & 93.8 \\
\hline & Input Cone & 23.0 & 26.5 & 28.5 & 31.0 & 29.5 & 30.2 & 32.9 & 34.4 \\
\hline \multirow{3}{*}{$\begin{array}{l}35.406 \text { nets } \\
1.636 \text { flipflops }\end{array}$} & Sensitive Input Cone & 12.0 & 14.4 & 17.8 & 21.2 & 25.0 & 28.9 & 33.1 & 36.6 \\
\hline & Output Cone & 6.12 & 9.81 & 9.14 & 21.3 & 22.8 & 24.1 & $2 \pi .3$ & 31.8 \\
\hline & Sensitive Output Cone & 3.56 & 7.11 & 9.49 & 13.6 & 16.7 & 21.0 & 24.2 & 28.8 \\
\hline \multirow{3}{*}{ s35932 } & $\operatorname{Ranc}$ & 46.3 & 71.1 & 84.2 & 91.1 & 95.0 & $9 \div .2$ & 98.9 & 99.2 \\
\hline & Str & 21.0 & 46.5 & 70.5 & 80.8 & 84.8 & $8 \pi .0$ & 89.9 & 91.1 \\
\hline & Input & 20.4 & 30.6 & 38.5 & 35.9 & 33.1 & $33 . i$ & $35 . i$ & 38.0 \\
\hline \multirow{3}{*}{$\begin{array}{l}32.045 \text { nets } \\
1.728 \text { flipflops }\end{array}$} & Sensitive Input Cone & 26.7 & 30.1 & $32 . \bar{T}$ & 32.6 & $34 . \overline{7}$ & $3 i .6$ & 39.4 & 41.0 \\
\hline & Output Cone & 10.2 & 16.9 & 21.4 & 24.9 & 28.3 & 28.9 & 33.4 & 40.8 \\
\hline & Sensitive Output Cone & 9.00 & 16.1 & 22.2 & 27.2 & 28.9 & 32.9 & 34.7 & 38.8 \\
\hline \multirow{3}{*}{ s15850 } & Random & 47.2 & $\overline{72.4}$ & 85.3 & $\overline{91 . \bar{t}}$ & 95.5 & 97.3 & 98.4 & 99.1 \\
\hline & String & 17.1 & 35.2 & 63.6 & 80.6 & 87.8 & 91.2 & 93.6 & 94.6 \\
\hline & Input $C$ & 16.4 & 25.2 & 29.3 & 31.6 & 30.3 & 30.6 & 31.4 & 35.1 \\
\hline \multirow{3}{*}{$\begin{array}{c}14.926 \text { nets } \\
597 \text { Hipflops }\end{array}$} & Sensitive Input Cone & 13.0 & 15.0 & 18.2 & 24.2 & 26.4 & $2 \bar{i} . \bar{i}$ & 30.6 & 35.6 \\
\hline & Output Cone & 6.41 & 9.06 & 14.9 & 19.3 & 25.2 & 27.9 & $31 . \bar{\imath}$ & 37.3 \\
\hline & Sensitive Output Cone & 4.62 & 8.35 & 12.2 & 14.0 & 17.6 & 22.7 & $2 \pi .7$ & 35.4 \\
\hline
\end{tabular}

Table C.1: Effect of the partitioning on size of the cutset 


\begin{tabular}{|c|c||c|c|c|c|c|}
\hline \multirow{2}{*}{ Metric } & \multicolumn{1}{c||}{ Partitioning } & \multicolumn{4}{c|}{ \# of processors } \\
\cline { 3 - 7 } & Algorithm & 5 & 10 & 15 & 20 & 25 \\
\hline \hline \multirow{4}{*}{ Simulation Time } & Random & 74 & 66 & 58 & 53 & 51 \\
(in seconds) & String & 73 & 62 & 55 & 47 & 45 \\
& Input Cone & 75 & 64 & 55 & 52 & 50 \\
& Sensitive Input Cone & 74 & 64 & 56 & 50 & 47 \\
& Output Cone & 74 & 62 & 57 & 49 & 46 \\
& Sensitive Output Cone & 73 & 61 & 56 & 45 & 43 \\
\hline & Random & 79.5 & 88.9 & 87.4 & 88.8 & 87.0 \\
Inter-Processoryyyyyy & String & 58.1 & 61.5 & 67.3 & 66.8 & 71.4 \\
Communications & Input Cone & 18.1 & 13.4 & 19.3 & 22.9 & 26.2 \\
(in percent) & Sensitive Input Cone & 43.8 & 40.1 & 43.8 & 44.7 & 46.2 \\
& Output Cone & 29.5 & 38.2 & 41.1 & 43.3 & 44.8 \\
& Sensitive Output Cone & 35.1 & 32.6 & 34.4 & 32.2 & 35.5 \\
\hline \multirow{4}{*}{ Rolled back } & Random & 22.6 & 30.5 & 36.3 & 34.8 & 29.6 \\
events & String & 2.32 & 6.64 & 9.50 & 6.94 & 6.77 \\
(in percent) & Input Cone & 5.68 & 2.37 & 2.78 & 9.92 & 14.4 \\
& Sensitive Input Cone & 6.31 & 9.66 & 10.2 & 10.7 & 6.54 \\
& Output Cone & 1.72 & 2.39 & 2.69 & 3.23 & 3.99 \\
& Sensitive Output Cone & 17.3 & 18.8 & 20.3 & 20.6 & 18.8 \\
\hline \multirow{2}{*}{ Load } & Random & 14.2 & 15.4 & 19.0 & 15.9 & 20.5 \\
inbalance & String & 74.1 & 86.3 & 91.6 & 93.9 & 94.8 \\
(in percent) & Input Cone & 98.6 & 99.8 & 99.7 & 99.5 & 99.4 \\
& Sensitive Input Cone & 90.2 & 90.1 & 91.9 & 91.0 & 86.7 \\
& Output Cone & 22.6 & 44.1 & 44.3 & 48.4 & 60.0 \\
& Sensitive Output Cone & 54.8 & 69.9 & 81.5 & 89.6 & 86.0 \\
\hline
\end{tabular}

Table C.2: Effect of partitioning on the simulation of circuit $s 15850$ 


\begin{tabular}{|c|c||c|c|c|c|c|}
\hline \multirow{2}{*}{ Metric } & \multicolumn{1}{|c||}{ Partitioning } & \multicolumn{5}{|c|}{ \# of processors } \\
\cline { 3 - 7 } & Algorithm & 5 & 10 & 15 & 20 & 25 \\
\hline \hline & Random & 86 & 62 & 51 & 49 & 50 \\
Simulation Time & String & 81 & 55 & 47 & 44 & 42 \\
(in seconds) & Input Cone & 89 & 62 & 50 & 47 & 45 \\
& Sensitive Input Cone & 85 & 60 & 47 & 45 & 46 \\
& Output Cone & 82 & 58 & 49 & 47 & 46 \\
& Sensitive Output Cone & 78 & 58 & 43 & 42 & 40 \\
\hline & Random & 76.6 & 81.2 & 87.7 & 88.2 & 89.5 \\
Inter-Processor & String & 59.5 & 62.6 & 68.6 & 72.8 & 77.1 \\
Communications & Input Cone & 12.0 & 20.9 & 33.5 & 41.2 & 48.5 \\
(in percent) & Sensitive Input Cone & 55.2 & 57.7 & 66.5 & 67.3 & 68.4 \\
& Output Cone & 45.3 & 48.3 & 54.2 & 55.1 & 55.8 \\
& Sensitive Output Cone & 48.8 & 52.5 & 60.6 & 40.2 & 38.1 \\
\hline & Random & 15.2 & 23.5 & 29.6 & 26.3 & 25.5 \\
Rolled back & String & 3.23 & 5.02 & 7.34 & 6.54 & 6.33 \\
events & Input Cone & 6.09 & 10.3 & 9.70 & 10.6 & 8.71 \\
(in percent) & Sensitive Input Cone & 5.32 & 5.31 & 6.35 & 7.27 & 7.13 \\
& Output Cone & 2.18 & 4.21 & 5.74 & 4.81 & 4.87 \\
& Sensitive Output Cone & 7.21 & 12.6 & 12.2 & 15.1 & 12.9 \\
\hline & Random & 50.5 & 31.6 & 24.2 & 23.6 & 25.7 \\
Load & String & 84.4 & 86.2 & 89.6 & 91.5 & 90.8 \\
inbalance & Input Cone & 98.9 & 98.3 & 93.6 & 96.6 & 95.6 \\
(in percent) & Sensitive Input Cone & 82.5 & 81.3 & 84.2 & 88.2 & 81.9 \\
& Output Cone & 74.3 & 58.6 & 55.4 & 52.3 & 57.3 \\
& Sensitive Output Cone & 63.6 & 61.6 & 63.4 & 61.3 & 58.1 \\
\hline
\end{tabular}

Table C.3: Effect of partitioning on the simulation of circuit s35932 


\begin{tabular}{|c|c|c|c|c|c|c|}
\hline \multirow[b]{2}{*}{ Metric } & \multirow{2}{*}{$\begin{array}{l}\text { Partitioning } \\
\text { Algorithm }\end{array}$} & \multicolumn{5}{|c|}{ \# of processors } \\
\hline & & 5 & 10 & 15 & 20 & 25 \\
\hline \multirow{6}{*}{$\begin{array}{l}\text { Simulation Time } \\
\text { (in seconds) }\end{array}$} & Random & 122 & 79 & 61 & 56 & 54 \\
\hline & String & 102 & 67 & 49 & 45 & 45 \\
\hline & Input Cone & 120 & 80 & 59 & 52 & 49 \\
\hline & Sensitive Input Cone & 117 & 75 & 60 & 50 & 47 \\
\hline & Output Cone & 109 & 71 & 58 & 49 & 46 \\
\hline & Sensitive Output Cone & 103 & 65 & 45 & 42 & 41 \\
\hline \multirow{6}{*}{$\begin{array}{c}\text { Inter-Processor } \\
\text { Communications } \\
\text { (in percent) }\end{array}$} & Random & 78.9 & 82.3 & 90.7 & 92.6 & 94.2 \\
\hline & String & 46.9 & 52.3 & 59.8 & 65.6 & 84.9 \\
\hline & Input Cone & 5.44 & 6.54 & 8.21 & 12.3 & $29 . \bar{T}$ \\
\hline & Sensitive Input Cone & 65.8 & 65.2 & 63.5 & 68.8 & 70.4 \\
\hline & Output Cone & 51.5 & 58.3 & 66.0 & 65.4 & 64.3 \\
\hline & Sensitive Output Cone & 55.0 & 57.5 & 63.9 & 62.6 & 63.5 \\
\hline \multirow{6}{*}{$\begin{array}{l}\text { Rolled back } \\
\text { events } \\
\text { (in percent) }\end{array}$} & Random & $\overline{25.7}$ & 28.1 & 36.0 & 37.3 & 39.0 \\
\hline & String & 7.39 & 9.40 & 10.5 & 8.35 & 8.18 \\
\hline & Input Cone & 8.19 & 8.35 & 8.72 & 9.82 & 8.98 \\
\hline & Sensitive Input Cone & 6.08 & 8.32 & 8.49 & 9.01 & 8.91 \\
\hline & Output Cone & 4.07 & 4.83 & 5.02 & 4.94 & 5.03 \\
\hline & Sensitive Output Cone & 9.02 & 10.6 & 11.0 & 11.7 & 12.0 \\
\hline \multirow{6}{*}{$\begin{array}{l}\text { Load } \\
\text { inbalance } \\
\text { (in percent) }\end{array}$} & Random & 72.7 & $\overline{24.6}$ & 18.6 & 18.2 & 17.4 \\
\hline & String & 86.2 & 86.6 & 89.7 & 87.2 & 85.3 \\
\hline & Input Cone & 98.9 & 99.0 & 99.1 & 99.3 & 99.6 \\
\hline & Sensitive Input Cone & 84.7 & 85.5 & 87.9 & 91.2 & 94.7 \\
\hline & Output Cone & $57 . \vec{t}$ & 61.8 & 64.1 & 70.3 & 75.1 \\
\hline & Sensitive Output Cone & 79.0 & 72.5 & 74.1 & 71.9 & $7 \pi .1$ \\
\hline
\end{tabular}

Table C.4: Effect of partitioning on the simulation of circuit s38417 


\begin{tabular}{|c|c||c|c|c|c|c|}
\hline \multirow{3}{*}{ Metric } & \multicolumn{1}{|c||}{ Partitioning } & \multicolumn{5}{c|}{ \# of processors } \\
\cline { 3 - 7 } & Algorithm & 5 & 10 & 15 & 20 & 25 \\
\hline \hline \multirow{4}{*}{ Simulation Time } & Random & 108 & 90 & 88 & 85 & 84 \\
(in seconds) & String & 104 & 91 & 76 & 73 & 71 \\
& Input Cone & 110 & 96 & 88 & 81 & 78 \\
& Sensitive Input Cone & 107 & 93 & 81 & 78 & 76 \\
& Output Cone & 105 & 90 & 76 & 74 & 73 \\
& Sensitive Output Cone & 102 & 88 & 72 & 69 & 66 \\
\hline & Random & 78.5 & 89.2 & 93.1 & 94.2 & 96.4 \\
Inter-Processor & String & 57.8 & 59.5 & 75.6 & 81.3 & 83.6 \\
Communications & Input Cone & 15.6 & 18.5 & 24.5 & 38.6 & 44.8 \\
(in percent) & Sensitive Input Cone & 70.8 & 72.2 & 75.7 & 77.9 & 79.8 \\
& Output Cone & 57.3 & 60.6 & 62.2 & 66.4 & 69.3 \\
& Sensitive Output Cone & 64.3 & 65.1 & 65.0 & 65.9 & 66.3 \\
\hline \multirow{4}{*}{ Rolled back } & Random & 21.6 & 26.3 & 31.4 & 31.8 & 29.6 \\
events & String & 5.82 & 8.21 & 10.7 & 11.1 & 10.2 \\
(in percent) & Input Cone & 8.65 & 8.73 & 9.32 & 10.5 & 7.29 \\
& Sensitive Input Cone & 6.31 & 7.15 & 7.28 & 8.22 & 7.01 \\
& Output Cone & 2.65 & 3.48 & 3.97 & 4.10 & 4.02 \\
& Sensitive Output Cone & 11.3 & 12.6 & 14.8 & 14.9 & 16.2 \\
\hline \multirow{2}{*}{ Load } & Random & 44.8 & 19.9 & 21.0 & 23.5 & 22.3 \\
inbalance & String & 94.7 & 91.8 & 94.2 & $8 i .6$ & 85.4 \\
(in percent) & Input Cone & 99.3 & 99.2 & 99.3 & 99.4 & 99.6 \\
& Sensitive Input Cone & 90.6 & 89.1 & 88.4 & 80.3 & 79.6 \\
& Output Cone & 49.5 & 50.8 & 44.9 & 58.4 & $56 . i$ \\
& Sensitive Output Cone & 56.3 & 54.5 & 51.1 & 49.8 & 46.8 \\
\hline
\end{tabular}

Table C.5: Effect of partitioning on the simulation of circuit s38584 


\begin{tabular}{|c||c|c|c|c|}
\hline $\begin{array}{c}\text { Partitioning } \\
\text { algorithm }\end{array}$ & $\begin{array}{c}\text { IPC } \\
(\%)\end{array}$ & $\begin{array}{c}\text { Inbalance } \\
(\%)\end{array}$ & $\begin{array}{c}\text { Rollbacks } \\
(\%)\end{array}$ & $\begin{array}{c}\text { Simulation time } \\
\text { (seconds) }\end{array}$ \\
\hline Random & 95.3 & 27.8 & 31.4 & 84 \\
String & 63.2 & 51.4 & 27.2 & 71 \\
Input & 33.3 & 78.6 & 17.2 & 77 \\
Sensitive Input & 60.2 & 55.1 & 15.4 & 76 \\
Output & 68.6 & 45.8 & 7.91 & 77 \\
Sensitive Output & 70.2 & 37.3 & 16.5 & 65 \\
\hline
\end{tabular}

Table C.6: Effect of Random Mapping on the simulation of circuit s38584

\begin{tabular}{|c||c|c|c|c|}
\hline $\begin{array}{c}\text { Partitioning } \\
\text { algorithm }\end{array}$ & $\begin{array}{c}\text { IPC } \\
(\%)\end{array}$ & $\begin{array}{c}\text { Inbalance } \\
(\%)\end{array}$ & $\begin{array}{c}\text { Rollbacks } \\
(\%)\end{array}$ & $\begin{array}{c}\text { Simulation time } \\
\text { (seconds) }\end{array}$ \\
\hline Random & 94.5 & 23.2 & 31.9 & 86 \\
String & 50.7 & 87.9 & 11.4 & 74 \\
Input & 38.3 & 99.4 & 10.6 & 81 \\
Sensitive Input & $7 . .4$ & 80.2 & 8.26 & 77 \\
Output & 66.4 & 58.5 & 4.15 & 74 \\
Sensitive Output & 66.2 & 49.9 & 15.0 & 70 \\
\hline
\end{tabular}

Table C. 7 : Effect of Vertical Natural Mapping on the simulation of circuit s38584

\begin{tabular}{|c||c|c|c|c|}
\hline $\begin{array}{c}\text { Partitioning } \\
\text { algorithm }\end{array}$ & $\begin{array}{c}\text { IPC } \\
(\%)\end{array}$ & $\begin{array}{c}\text { Inbalance } \\
(\%)\end{array}$ & $\begin{array}{c}\text { Rollbacks } \\
(\%)\end{array}$ & $\begin{array}{c}\text { Simulation time } \\
\text { (seconds) }\end{array}$ \\
\hline Random & 96.8 & 25.5 & 29.7 & 85 \\
String & 63.9 & 33.4 & 31.4 & 69 \\
Input & 33.3 & 55.1 & 17.5 & 78 \\
Sensitive Input & 58.6 & 35.7 & 16.8 & 78 \\
Output & 68.6 & 42.6 & 12.9 & 76 \\
Sensitive Output & 69.5 & 41.5 & 17.8 & 67 \\
\hline
\end{tabular}

Table C.8: Effect of Horizontal Natural Mapping on the simulation of circuit s38584 


\section{Appendix D}

\section{Quantitative Results of Queueing Network Simulations}

These tables present the performance results of the simulation of queueing network models. for both the Time Warp and the Clustered Time Warp algorithm. Three different networks were used: a pipeline (tables D.1 and D.2). a hierarchical structure (tables D.3 and D.4) and a distributed network (tables D.5 and D.6).

The size of the models ranged from 9.000 to 58.800 processes and the simulations ran on 15 processors for the pipeline model and 17 processors for both the hierarchical and the distributed models. Three different service time distributions were used: deterministic, uniform and exponential. For each simulation the throughput, the memory usage and the rollbacks were measured. The throughput is defined as being the total number of committed events per second. The memory usage is the maximum memory requirement of any processor to complete the simulation. The rollback metric indicates the proportion of rolled back events compared to the total number of events processed. For each configuration. the simulation was ran three times and the average of the measurements was calculated (appears in bold characters in the tables). These results are discussed in chapter $T$. 


\begin{tabular}{|c|c|c|c|c|c|c|c|c|c|}
\hline \multirow{2}{*}{$\begin{array}{l}\text { Model size } \\
\text { (\# nodes) }\end{array}$} & \multicolumn{3}{|c|}{$\begin{array}{c}\text { Throughput } \\
\text { (events/s) }\end{array}$} & \multicolumn{3}{|c|}{$\begin{array}{c}\text { Memory Usage } \\
\text { (KBytes) }\end{array}$} & \multicolumn{3}{|c|}{$\begin{array}{c}\text { Rollbacks } \\
\text { (\%) }\end{array}$} \\
\hline & $\overline{\text { Det }}$ & $U n i$ & $E_{x p}$ & $\overline{\text { Det }}$ & $U n i$ & $E x p$ & Det & $U n i$ & $E x p$ \\
\hline \multirow{4}{*}{9,000} & 3435 & 3378 & 3659 & 1305 & 1113 & 1169 & 11 & 12 & 12 \\
\hline & 3512 & 3354 & 3580 & 1369 & 1083 & 1162 & 11 & 11 & 11 \\
\hline & 3497 & 3392 & 9622 & 1264 & 1078 & 1108 & 11 & 13 & 11 \\
\hline & 3481 & $\overline{3375}$ & 3620 & 1313 & 1081 & 1146 & 11 & 12 & 11 \\
\hline \multirow{4}{*}{18.000} & 2642 & 3080 & 3122 & 1535 & 1356 & 1426 & 17 & 16 & 15 \\
\hline & 2685 & 2925 & 3084 & 1581 & 1318 & 1431 & 15 & 17 & 14 \\
\hline & 2620 & 2992 & 3105 & 1495 & 1320 & 1426 & 15 & 16 & 15 \\
\hline & 2649 & 2999 & 3104 & 1537 & 1331 & 1428 & 16 & 16 & 15 \\
\hline \multirow{4}{*}{27,000} & 2298 & 2489 & 2284 & 1471 & 1509 & 1417 & 25 & 23 & 24 \\
\hline & 2295 & 2512 & 2325 & 1538 & 1525 & 1506 & 24 & 24 & 25 \\
\hline & 2279 & 2457 & 2295 & 1525 & 1512 & 1428 & 24 & 23 & 24 \\
\hline & 2291 & 2486 & 2301 & 1511 & 1515 & 1450 & 24 & 23 & 24 \\
\hline \multirow{4}{*}{36.000} & 1820 & 1863 & 1812 & 1520 & 1510 & 1497 & 36 & 33 & 34 \\
\hline & 1815 & 1846 & 1821 & 1553 & 1498 & 1521 & 34 & 35 & 35 \\
\hline & 1823 & 1834 & 1825 & 1546 & 1508 & 1514 & 35 & 33 & 35 \\
\hline & 1819 & 1848 & 1820 & 1539 & 1505 & 1511 & $\overline{35}$ & 34 & $\overline{35}$ \\
\hline
\end{tabular}

Table D.1: Pipeline model - Time Warp

\begin{tabular}{|c|c|c|c|c|c|c|c|c|c|}
\hline \multirow{2}{*}{$\begin{array}{c}\text { Model size } \\
\text { (\# nodes) }\end{array}$} & \multicolumn{3}{|c|}{$\begin{array}{c}\text { Throughput } \\
\text { (events/s) }\end{array}$} & \multicolumn{3}{|c|}{$\begin{array}{c}\text { Memory Usage } \\
\text { (KBytes) }\end{array}$} & \multicolumn{3}{|c|}{$\begin{array}{c}\text { Rollbacks } \\
(\%)\end{array}$} \\
\hline & Det & $U n i$ & $E x p$ & $\overline{\text { Det }}$ & $U n i$ & $E x p$ & $\overline{D e t}$ & $U n i$ & $E x p$ \\
\hline \multirow{4}{*}{$\begin{array}{c}9.000 \\
300 \text { per cluster }\end{array}$} & 3675 & 3635 & 3584 & 356 & 370 & 407 & 7 & 5 & 6 \\
\hline & 3624 & 3585 & 3595 & 371 & 362 & 400 & 7 & 6 & 6 \\
\hline & 3598 & 3612 & 3607 & 361 & 357 & 352 & 7 & 5 & 5 \\
\hline & 3632 & 3611 & 3595 & 363 & 363 & 386 & 7 & 5 & 6 \\
\hline \multirow{4}{*}{$\begin{array}{c}18.000 \\
600 \text { per cluster }\end{array}$} & 3591 & 3275 & 3025 & 462 & 479 & 496 & 8 & 4 & 5 \\
\hline & 3552 & 3308 & 3107 & 458 & 481 & 488 & 7 & 5 & 6 \\
\hline & 3586 & 3285 & 3091 & 461 & 472 & 529 & 8 & 5 & 5 \\
\hline & $\overline{3576}$ & 3289 & 3074 & 460 & 477 & 504 & 8 & 5 & 5 \\
\hline \multirow{4}{*}{$\begin{array}{c}27.000 \\
900 \text { per cluster }\end{array}$} & 3425 & 3273 & 3012 & 556 & 538 & 508 & 7 & 5 & 7 \\
\hline & 3414 & 3260 & 2987 & 518 & 545 & 524 & 8 & 6 & 8 \\
\hline & 3430 & 3204 & 2999 & 524 & 548 & 517 & 8 & 7 & 6 \\
\hline & 3423 & 3246 & 2999 & 533 & 544 & $\overline{516}$ & 8 & 6 & 7 \\
\hline \multirow{4}{*}{$\begin{array}{c}36,000 \\
1,200 \text { per cluster }\end{array}$} & 3381 & 3190 & 3002 & 584 & 559 & 562 & 9 & 7 & 8 \\
\hline & 3354 & 3183 & 2983 & 576 & 572 & 566 & 8 & 7 & 8 \\
\hline & 3374 & 3167 & 2991 & 578 & 567 & 559 & 8 & 6 & 7 \\
\hline & 3370 & 3180 & 2992 & 579 & 566 & 562 & 8 & 7 & 8 \\
\hline
\end{tabular}

Table D.2: Pipeline model - Clustered Time Warp 


\begin{tabular}{|c|c|c|c|c|c|c|c|c|c|}
\hline \multirow{2}{*}{$\begin{array}{l}\text { Model size } \\
\text { (\# nodes) }\end{array}$} & \multicolumn{3}{|c|}{$\begin{array}{c}\text { Throughput } \\
\text { (events/s) }\end{array}$} & \multicolumn{3}{|c|}{$\begin{array}{c}\text { Memory Usage } \\
\text { (KBytes) }\end{array}$} & \multicolumn{3}{|c|}{$\begin{array}{c}\text { Rollbacks } \\
(\%)\end{array}$} \\
\hline & $\overline{D e t}$ & $U n i$ & $E x p$ & $\overline{\text { Det }}$ & $U n i$ & $E x p$ & $\overline{D e t}$ & $\overline{U n i}$ & $E x p$ \\
\hline \multirow{4}{*}{14.700} & 3670 & 3588 & 3533 & 1333 & 1616 & 1548 & 15 & 15 & 15 \\
\hline & 3584 & 3649 & 3580 & 1495 & 1523 & 1437 & 15 & 16 & 15 \\
\hline & 3630 & 3524 & 3565 & 1398 & 1586 & 1521 & 16 & 16 & 15 \\
\hline & 3628 & 3587 & 3560 & 1409 & 1575 & 1502 & $\overline{15}$ & 16 & 15 \\
\hline \multirow{4}{*}{29.400} & 3425 & 3368 & 3416 & 1606 & 1587 & 1632 & 18 & 19 & 20 \\
\hline & 3393 & 3317 & 3381 & 1515 & 1616 & 1560 & 19 & 19 & 19 \\
\hline & 3412 & 3369 & 3399 & 1670 & 1686 & 1605 & 18 & 18 & 20 \\
\hline & 3410 & 3351 & 3399 & 1597 & 1630 & 1599 & 18 & 19 & 20 \\
\hline \multirow{4}{*}{44.100} & 3010 & 2991 & 2978 & 1687 & 1710 & 1732 & 23 & 25 & 25 \\
\hline & 2988 & 2954 & 2987 & 1706 & 1679 & 1705 & 25 & 25 & 26 \\
\hline & 3023 & 2982 & 3012 & 1654 & 1682 & 1690 & 24 & 24 & 25 \\
\hline & 3007 & 2976 & 2992 & 1682 & 1690 & 1709 & 24 & 25 & 25 \\
\hline \multirow{4}{*}{58.800} & 2627 & 2595 & 2529 & 1687 & 1649 & 1793 & 26 & 29 & 28 \\
\hline & 2660 & 2552 & 2523 & 1675 & 1654 & 1712 & 27 & 28 & 28 \\
\hline & 2583 & 2464 & 2502 & 1717 & 1699 & 1736 & 27 & 29 & 28 \\
\hline & 2623 & 2537 & 2518 & 1693 & 1667 & 1747 & 27 & 29 & 28 \\
\hline
\end{tabular}

Table D.3: Hierarchical network model - Time Warp

\begin{tabular}{|c|c|c|c|c|c|c|c|c|c|}
\hline \multirow{2}{*}{$\begin{array}{l}\text { Model size } \\
\text { (\# nodes) }\end{array}$} & \multicolumn{3}{|c|}{$\begin{array}{c}\text { Throughput } \\
\text { (events/s) }\end{array}$} & \multicolumn{3}{|c|}{$\begin{array}{c}\text { Memory Usage } \\
\text { (KBytes) }\end{array}$} & \multicolumn{3}{|c|}{$\begin{array}{c}\text { Rollbacks } \\
(\%)\end{array}$} \\
\hline & Det & Uni & $E x p$ & Det & Uni & $E x p$ & $\overline{D e t}$ & $U n i$ & $E x p$ \\
\hline \multirow{4}{*}{$\begin{array}{c}14.700 \\
300 \text { per cluster }\end{array}$} & 3423 & 3387 & 3452 & 752 & 761 & 788 & 10 & 8 & 8 \\
\hline & 3488 & 3467 & 3412 & 702 & 698 & 751 & 11 & 9 & 8 \\
\hline & 3453 & 3392 & 3400 & 689 & 705 & 769 & 10 & 9 & 8 \\
\hline & 3455 & 3415 & 3421 & 714 & 721 & 769 & 10 & 9 & 8 \\
\hline \multirow{4}{*}{$\begin{array}{l}29.400 \\
600 \text { per cluster }\end{array}$} & 3385 & 3365 & 3402 & 789 & 792 & 812 & 12 & 10 & 10 \\
\hline & 3402 & 3378 & 3375 & 806 & 814 & 817 & 11 & 11 & 9 \\
\hline & 3395 & 3384 & 3393 & 810 & 783 & 820 & 12 & 10 & 9 \\
\hline & 3394 & $\overline{3376}$ & $\overline{3390}$ & 802 & 796 & 816 & 12 & 10 & 9 \\
\hline \multirow{4}{*}{$\begin{array}{c}44,100 \\
900 \text { per cluster }\end{array}$} & 3295 & 3301 & 3296 & 836 & 805 & 829 & 12 & 13 & 11 \\
\hline & 3254 & 3312 & 3300 & 830 & 814 & 832 & 14 & 11 & 12 \\
\hline & 3298 & 3308 & 3324 & 814 & 811 & 830 & 13 & 11 & 12 \\
\hline & 3282 & 3307 & 3307 & 827 & 810 & 830 & 13 & 12 & 12 \\
\hline \multirow{4}{*}{$\begin{array}{c}58,800 \\
1,200 \text { per cluster }\end{array}$} & 3096 & 3208 & 3308 & 845 & 857 & 855 & 15 & 14 & 13 \\
\hline & 3152 & 3258 & 3267 & 856 & 808 & 865 & 15 & 15 & 14 \\
\hline & 3161 & 3284 & 3259 & 860 & 812 & 885 & 16 & 15 & 14 \\
\hline & $\overline{3136}$ & 3250 & 3278 & 854 & 826 & 868 & 15 & 15 & 14 \\
\hline
\end{tabular}

Table D.4: Hierarchical network model - Clustered Time Warp 


\begin{tabular}{|c|c|c|c|c|c|c|c|c|c|}
\hline \multirow{2}{*}{$\begin{array}{l}\text { Model size } \\
\text { (\# nodes) }\end{array}$} & \multicolumn{3}{|c|}{$\begin{array}{c}\text { Throughput } \\
\text { (events/s) }\end{array}$} & \multicolumn{3}{|c|}{$\begin{array}{c}\text { Memory Usage } \\
\text { (KBytes) }\end{array}$} & \multicolumn{3}{|c|}{$\begin{array}{l}\text { Rollbacks } \\
\text { (\%) }\end{array}$} \\
\hline & $\overline{D e t}$ & $U n i$ & $E x p$ & Det & $U_{n i}$ & $E x p$ & $\overline{D e t}$ & $U n i$ & $E x p$ \\
\hline \multirow{4}{*}{14.700} & $\overline{4424}$ & 4187 & 4161 & 1350 & 1284 & 1168 & 8 & 8 & 9 \\
\hline & 4406 & 4208 & 4023 & 1308 & 1265 & 1225 & 8 & 9 & 9 \\
\hline & 4412 & 4210 & 4102 & 1361 & 1250 & 1190 & 9 & 9 & 10 \\
\hline & 4414 & 4202 & 4095 & 1340 & 1266 & 1194 & 8 & 9 & 9 \\
\hline \multirow{4}{*}{29.400} & 3885 & 3851 & 3811 & 1516 & 1509 & 1425 & 18 & 19 & 19 \\
\hline & 3874 & 3883 & 3696 & 1506 & 1481 & 1473 & 19 & 19 & 18 \\
\hline & 3870 & 3905 & 3820 & 1510 & 1453 & 1509 & 18 & 17 & 19 \\
\hline & 3876 & 3880 & 3776 & 1511 & 1481 & 1469 & 18 & 18 & 19 \\
\hline \multirow{4}{*}{44.100} & 2735 & 2648 & 2630 & 1590 & 1610 & 1625 & 27 & 28 & 28 \\
\hline & 2718 & 2662 & 2630 & 1650 & 1673 & 1699 & 27 & 28 & 27 \\
\hline & 2684 & 2646 & 2717 & 1634 & 1660 & 1628 & 26 & 29 & 29 \\
\hline & 2712 & 2652 & 2659 & 1625 & 1648 & 1651 & 27 & 28 & 28 \\
\hline \multirow{4}{*}{58.800} & 1845 & 1698 & 1800 & 1623 & 1682 & 1607 & 39 & 40 & 41 \\
\hline & 1896 & 1761 & 1739 & 1649 & 1672 & 1637 & 41 & 41 & 42 \\
\hline & 1880 & 1752 & 1778 & 1633 & 1656 & 1692 & 43 & 40 & 41 \\
\hline & 1874 & 1737 & 1772 & 1635 & 1670 & 1645 & 41 & 40 & 41 \\
\hline
\end{tabular}

Table D.5: Distributed network model - Time Warp

\begin{tabular}{|c|c|c|c|c|c|c|c|c|c|}
\hline \multirow{2}{*}{$\begin{array}{c}\text { Model size } \\
\text { (\# nodes) }\end{array}$} & \multicolumn{3}{|c|}{$\begin{array}{c}\text { Throughput } \\
\text { (events/s) }\end{array}$} & \multicolumn{3}{|c|}{$\begin{array}{c}\text { Memory Usage } \\
\text { (KBytes) }\end{array}$} & \multicolumn{3}{|c|}{$\begin{array}{c}\text { Rollbacks } \\
(\%)\end{array}$} \\
\hline & Det & $U_{n i}$ & $E x p$ & Det & $U_{n i}$ & $E x p$ & $\overline{D e t}$ & $\overline{U n i}$ & $E x p$ \\
\hline \multirow{4}{*}{$\begin{array}{c}14.700 \\
300 \text { per cluster }\end{array}$} & 3483 & 3376 & 3387 & 655 & 717 & 690 & 15 & 16 & 18 \\
\hline & 3468 & 3381 & 3301 & 715 & 672 & 796 & 16 & 16 & 19 \\
\hline & 3355 & 3320 & 3246 & 686 & 778 & 721 & 15 & 15 & 18 \\
\hline & 3435 & 3359 & 3311 & 685 & 722 & 736 & 15 & $\overline{16}$ & $\overline{18}$ \\
\hline \multirow{4}{*}{$\begin{array}{l}29.400 \\
600 \text { per cluster }\end{array}$} & 3267 & 3308 & 3218 & 714 & 760 & 770 & 17 & 17 & 19 \\
\hline & 3235 & 3205 & 3259 & 795 & 702 & 757 & 16 & 18 & 20 \\
\hline & 3290 & 3270 & 3213 & 779 & 789 & 796 & 17 & 18 & 20 \\
\hline & 3264 & 3261 & 3230 & 763 & 750 & 774 & 17 & 18 & 20 \\
\hline \multirow{4}{*}{$\begin{array}{l}44.100 \\
900 \text { per cluster }\end{array}$} & 3042 & 3100 & 3037 & 808 & 803 & 849 & 20 & 19 & 18 \\
\hline & 3101 & 3001 & 2960 & 767 & 845 & 888 & 20 & 19 & 19 \\
\hline & 3030 & 2985 & 3002 & 816 & 825 & 875 & 19 & 19 & 19 \\
\hline & 3058 & 3029 & 3000 & 797 & 824 & 871 & 20 & 19 & 19 \\
\hline \multirow{4}{*}{$\begin{array}{c}58,800 \\
1,200 \text { per cluster }\end{array}$} & 2882 & 2815 & 2825 & 898 & 955 & 888 & 22 & 22 & 23 \\
\hline & 2866 & 2802 & 2812 & 903 & 920 & 914 & 23 & 22 & 23 \\
\hline & 2934 & 2841 & 2796 & 871 & 907 & 953 & 22 & 24 & 24 \\
\hline & 2894 & 2819 & $\overline{2811}$ & $\overline{891}$ & 927 & 918 & 22 & 23 & 23 \\
\hline
\end{tabular}

Table D.6: Distributed network model - Clustered Time Warp 


\section{Appendix E}

\section{Relevant World Wide Web sites}

This list gives major entry points of the World Wide Web relevant to this thesis.

www.cs.utsa.edu/research/ParSim Home page of the Parallel and Distributed Simulation research community maintained by Samir $R$. Das at the University of Texas at San Antonio.

may.cs.ucla.edu Research led by Rajive Bagrodia at UCLA.

www.cc.gatech.edu/computing/Architecture/projects/pads.html Research on Time Warp based systems led by Richard Fujimoto at Georgia Tech.

www.ece.uc.edu/ paw/lab The Computer Architecture Design Laboratory led by Philip IVilsey at the Lniversity of Cincinnati.

www.it.kth.se/labs/sim The Simulation Laboratory led by Rassul Ayani at the Roval Institute of Technology. Sweden.

www.dcs.exeter.ac.uk/research/parallel.html The Parallel Systems Research Laboratory led by Stephen J. Turner at the University of Exeter. England.

bungee.cpsc.ucalgary.ca The Time Warp group at the Lniversity of Calgary led by Brian Unger.

www.cs.waikato.ac.nz/timestamp The Time Warp group led by John Cleary at the Lniversity of Waikato. New Zealand.

www-dsg.stanford.edu/paradise.html The PARADISE project (Performance ARchitecture for Advanced Distributed Interactive Simulation Environments) led by David Cheriton at Stanford University.

www.pads.uwaterloo.ca The Parallel and Distributed Simulation research group at the University of Waterloo led by Bruno R. Preiss.

www.cs.wisc.edu/ wwt The Winsconsin Wind Tunnel Project. 
dictator.uwaterloo.ca/pads96 Home page of the 10th ACM/IEEE/SCS Workshop on Parallel and Distributed Simulation.

www.cc.ukans.edu/wsc96 Home page of the 1996 Winter Simulation Conference.

www.acm.org/pubs/tomacs Home page of the ACM Transactions on Modeling and Computer Simulation.

www.bbn.com Home page of the BBN Corporation. 


\section{Bibliography}

[Agre89] J.R. Agre. "Simulation of Time Warp Distributed Simulations". Proceedings of the Distributed Simulation Conference, SCS simulation series, Vol.21, No.2, 1989. pp85-90.

[Avri95] H. Avril, C. Tropper. "Clustered Time Warp and Logic Simulation". Proceedings of the 9th Workshop on Parallel and Distributed Simulation, 1995. pp112-119.

[Avri96] H. Avril, C. Tropper, -The Dynamic Load Balancing of Clustered Time Warp for Logic Simulation". Proceedings of the 10th Workshop on Parallel and Distributed Simulation, 1996. pp20-27.

[Bagr91] R. Bagrodia. K.M. Chandy. W.-T. Liao, “An Experimental Study on the Performance of the Space-Time Simulation Algorithm". Proceedings of the 6th Workshop on Parallel and Distributed Simulation. 1992. pp159-168.

[Bagr91b] R.L. Bagrodia, W.-T. Liao. "Parallel Simulation of the Sharks World Problem". Proceedings of the Distributed Simulation Conference. SCS simulation series. Vol.22. No.1. 1990. pp191-198.

[Bail88] M.L. Bailey, L. Snyder, “An Empirical Study of On-Chip Parallelism”, 25th ACM/IEEE Design Automation Conference, 1988. pp160-165.

[Bail89] M.L. Bailey, L. Snyder. "The Effect of Timing on the Parallelism Available for Parallel Circuit Simulation", 3rd Workshop on Parallel and Distributed Simulation. 1989. pp157-163.

[Bail92] M.L. Bailey. Y.-B. Lin. “Synchronization Strategies for Parallel Logic-Level Simulation”. International Joumal Comput. Simulation. 1992, pp211-230.

[Bail92b] M.L. Bailey, “How Circuit Size Affects Parallelism”. IEEE Transactions on Computer-Aided Design. Vol.11. No.2. February 1992. pp208-215.

[Bail94] M.L. Bailey, J.V. Briner, R.D. Chamberlain. "Parallel Logic Simulation of VLSI Systems”. ACM Computing Surveys. Vol.26, No.3. September 1994, pp.255-295

[Barn92] E.R. Barnes, "An Algorithm for Partitioning the Nodes of a Graph". SIAM Journal on Algebraic and Discrete Methods. December 1982, pp541-550.

[Baue92] H. Bauer, C. Sporrer. "Distributed Logic Simulation and an Approach to Asynchronous GVTCalculation". Proceedings of the 6th Workshop on Parallel and Distributed Simulation. 1992, pp205208.

[Baum89] K. Baumgartner, et. al., -Implementation of Gammon: An Efficient Load Balancing Strategy for Local Computer System”. International Conference on Parallel Processing. 1989. pp83-92. 
[Bell90] S. Bellenot, “Global Virtual Time Algorithms”, Distributed Simulations, 1990, pp122-127.

[Berr85] O. Berry, D. Jefferson “Critical Path Analysis of Distributed Simulation”. Proceedings of the 1985 Distributed Simulation Conference, SCS simulation series, Vol.15, No.2, 1985, pp57-60.

[Berr86] O. Berry "Performance Evaluation of the Time Warp Distributed Simulation Mechanism". Ph.D. Dissertation, University of Southern California, Los Angeles. California. 1986.

[Bers88] B.N. Bershad, “The PRESTO User's Manual.”, Technical Report 88-01-04. Department of Computer Science, University of Washington, Seattle, WA.

[Bill93] W.D. Billowitch, “IEEE 1164: Helping designers share VHDL models”, IEEE Spectr. 30. June 1993. pp37.

[Bouk91] A. Boukerche, C. Tropper, "Performance Analysis of Distributed Simulation with Clustered Processes", Proc. 1991, SCS Multiconference on Advances in Distributed Simulation. Anaheim. California. January 1991, pp112-120.

[Bouk94] A. Boukerche, C. Tropper, -A Static Partitioning and Mapping Algorithm for Conservative Parallel Simulations", Proceedings of the 8th Workshop on Parallel and Distributed Simulation. 1994. pp164-172.

[Bouk95] A. Boukerche. "Parallel Simulation With Clustered Processes: Principle and Practice". Ph.D. Thesis, McGill University, Montreal, Canada, 1995.

[Bouk95b] A. Boukerche, C. Tropper, "SGTNE:Semi-Global Time of the Next Event Algorithm". Proceedings of the 9th Workshop on Parallel and Distributed Simulation. 1995. pp68-77.

[Brgl89] F. Brglez, D. Bryan, K. Kozminski, “Combinational Profiles of Sequential Benchmark Circuits", ISCAS'89, pp1929-1934.

[Brin89] J.V. Briner. Jr., “A framework for analyzing parallel discrete event simulation”. Proceedings of the Computer Measurement Group. CMG, Dallas, Texas, pp180-185.

[Brin90] J.V. Briner. Jr., "Parallel Mixed-Level Simulation of Digital Circuits Using Virtual Time". Ph.D. thesis. Duke University 1990.

[Brin91] J.V. Briner Jr.. "Fast Parallel Simulation of Digital Systems". Proceedings of the 5th Workshop on Parallel and Distributed Simulation, 1991, pp71-77.

[Brya77] R.E. Bryant, “Simulations of Packet Communication Architecture Computer Systems”. Technical Report 188, MIT, LCSi, 1977.

[Brya84] R.E. Bryant, “A Switch-Level Model and Simulator for MOS Digital Systems”. IEEE Trans. on Computers. Vol C-33, No.2. February 1984, pp160-177.

[Burd93] C. Burdorf. J. Marti, “Load Balancing Strategies for Time Warp on Multi-User Workstations”. The Computer Journal, Vol.36. No.2, 1993, pp168-176.

[Buzz90] C.V. Buzzell, M.J. Robb, R.M. Fujimoto, “Modular VME Rollback Hardware for Time Warp”, Proceedings of the 4th Workshop on Parallel and Distributed Simulation, 1990, pp153-156. 
[Byte95] Byte Magazine, November 1995. pl80.

[Cham94] R.D. Chamberlain, C.D. Henderson, EEvaluating the Use of Pre-Simulation in VLSI Circuit Partitioning", Proceedings of the 8th Workshop on Parallel and Distributed Simulation. 1994. pp139-146.

[Chan79] K. Chandy, J. Misra, -Distributed Simulation: A Case Study in Design and Verification of Distributed Programs”, IEEE Trans. Software Eng., S-5, September 1979. pp440-452.

[Chan83] K.M. Chandy, J. Misra, L.M. Haas, "Distributed Deadlock Detection”. ACM Trans. Comput. Syst., Vol.1, No.2, May 1983. pp144-156.

[Chan89] K. Chandy. R. Sherman, "Space-Time and Simulation”, Proceedings of the 1990 Distributed Simulation Conference, SCS simulation series, Vol.21, No.2, March 1989. pp53-57.

[Chun90] M.J. Chung. Y. Chung. -Data Parallel Simulation using Time-Warp on the Connection Machine". 26th ACM/IEEE Design Automation Conference. 1989. pp98-103.

[Cido87] I. Cidon, J.M. Jaffe, M. Sidi, -Local Distributed Deadlock Detection by Cycle Detection and Clustering”. IEEE Trans. Soft. Eng.. Vol. SE-13. No.1, January 1987. pp13-14.

[Conc91] A.I. Concepcion. S.G. Kelly. “Computing Global Virtual Time Using the Multi-Level Token Passing Algorithm”. Proceedings of the 5th Workshop on Parallel and Distributed Simulation. 1991. pp63-68.

[Conk90] D. Conklin. J. Cleary. B. Unger. "The Sharks World - A Study in Distributed Simulation Design". Proceedings of the 1990 Distributed Simulation Conference. SCS simulation series. Vol.22. No.1. 1990. pp157-160.

[Corm91] T.H. Cormen. C.E. Leiserson, R.L. Rivest, "Introduction to Algorithms”. The MIT electrical engineering and computer science series. McGraw-Hill.

[Coul94] G. Coulouris, J. Dollimore. T. Kindberg, “Distributed Systems: Concepts and Designs”. Published by Addison-Wesley, Second Edition, 1994.

[Das93] S.R. Das. R.M. Fujimoto, “A Performance Study of the Cancelback Protocol for Time Warp”. Proceedings of the 7th Workshop on Parallel and Distributed Simulation. 1993. pp135-142.

[Dey90] S. Dey. F. Brglez. G. Kedem. -Corolla Based Circuit Partitioning and Application to Logic Synthesis”. Technical Report 90-40. Duke University, 1990.

[Elma86] A. K. Elmagarmid, -A Survey of Distributed Deadlock Detection Algorithms". ACM SIGMOD Record. Vol.15. No.3. September 1986, pp37-45

[Farb73] D. J. Farber, et. al.. “The Distributed Computing System”. Proc. Compcon Spring 1973.

[Fidu82] C. M. Fiduccia, R. M. Mattheyses, -A Linear-Time Heuristic for Improving Network Partitions". Proceedings of the 19th Design Automation Conference, 1982. pp175-181.

[Fran85] E. H. Frank. -A data-driven multiprocessor for switch-level simulation of VLSI circuits”, Ph.D. thesis. Carnegie-Mellon University, November 1985. 
[Fran86] E.H. Frank, "Exploiting parallelism in a switch-level simulation machine`. Proceedings of the 23rd ACM/IEEE Design Autmoation Conference, IEEE, New York. pp20-26.

[Fuji88] R.M. Fujimoto. J.-J. Tsai, G. Gopalakrishnan, "The Roll Back Chip: Hardware Support for Distributed Simulation using Time Warp", 2nd Workshop on Parallel and Distributed Simulation. 1988, pp81-86.

[Fuji89] R.M. Fujimoto, "Time Warp on a Shared Memory Multiprocessor". Technical Report UUCS88-021a. University of Utah. January 1989.

[Fuji90] R.M. Fujimoto, "Performance of Time Warp Under Synthetic Workloads". Proceedings of the 4th Workshop on Parallel and Distributed Simulation 1990, pp23-28.

[Fuji90b] R.M. Fujimoto, “Parallel Discrete Event Simulation”, CACM, Vol.33. No.10, 1990. pp31-53.

[Gafn85] A. Gafni, “Space Management and Cancellation Mechanism for Time Warp”. Ph.D. thesis. University of Southern California. 1985.

[Gafn88] A. Gafni, “Rollback Mechanisms for Optimistic Distributed Simulation Systems”. 2nd Workshop on Parallel and Distributed Simulation, 1988, pp61-67.

[Ga084] C. Gao. et. al., "Load Balancing Algorithms in Homogeneous Distributed Systems". Proc. of the 1984 International Conference on Parallel Processing, August 1984. pp41-63.

[Giro91] P. Girodias. -De l’à-propos du modèle de simulation optimiste pour limplantation de VHDL dans un environnement informatique distribuém. M.Sc. thesis, Université de Montréal. 1991.

[Glan95] N.S. Glance. T. Hogg, "Dilemmas in Computational Societies”. First International Conference on Multiagent Systems. San Fransisco, California, June 1995.

[Glaz93] D.M. Glazer, “Load Balancing Parallel Discrete Event Simulations”. Ph.D. thesis. McGill University. 1993.

[Glaz93b] D.M. Glazer, C.Tropper. “A Dynamic Load Balancing Algorithm for Time Warp”. Parallel and Distributed Systems, Vol.4. No.3, March 1993, pp318-327.

[Goto77] S. Goto, I. Cederbaum. B.S. Ting, "Suboptimal Solution of the Backboard Ordering with Channel Capacity Constraint”. IEEE Trans. Circuits and Systems, November 1977, pp645-652.

[Gros88] B. Groselj, C. Tropper. “The Time-of-Next-Event Algorithm”. Proceedings of the 1988 Distributed Simulation Conference. SCS simulation series, Vol.19, No.3. February 1988, pp25-29.

[Gros88b] B. Groselj. "Pseudosimulation and the Distributed Simulation of Clustered Processes”. Ph.D. Thesis. McGill University, School of Computer Science, June 1988.

[Gros91] B. Groselj, C. Tropper, "The Distributed Simulation of Clustered Processes". Distributed Computing, Springer Verlag, Vol.4, pp111-121.

[Had192] S.W. Hadley, B.L. Mark. A. Vannelli, “An Efficient Eigenvector Approach for Finding Netlist Partitions”. IEEE Transactions on Computer-Aided Design, Vol.11. No.7, July 1992, pp15-23

[Hawk88] S. Hawking, “A Brief History of Time”, Bantam Books, New York, New York, 1988. 
[Hont89] P. Hontalasa, B. Beckman, M. DiLoreto, L. Blume, P. Reiher, K. Sturdevant. L. Van Warren, J. Wedel, F. Wieland, D. Jefferson, "Performance of the Colliding Pucks Simulation on the Time Warp Operating System", Proceedings of the Distributed Simulation Conference. SCS simulation series, Vol.21, No.2, 1989, pp3-7.

[Hube93] , B.A. Huberman, T. Hogg, “The Emergence of Computational Ecologies", 1992 Lectures in Complex Systems, Edited by Lynn Nadel and Daniel Stein. SFI Studies in the Sciences of Complexity, Addison-Wesley, 1993, pp185-205.

[IEEE88] , IEEE, “IEEE Standard VHDL Language Reference Manual”. New York, New York, 1988.

[Jeff85] D. Jefferson, "Virtual Time”, ACM Trans. Prog. Lang. Syst, Vol.7. No.3, July 1985, pp404-425.

[Jeff85b] D. Jefferson, B. Beckman, S. Hughes, E. Levy, T. Litwin, J. Spagnuolo. J. Vavrus, F. Wieland, B. Zimmerman, "Implementation of Time Warp on the Caltech Hypercube". Proceedings of the 1985 Distributed Simulation Conference, SCS simulation series, Vol.15. No.2, 1985, pp70-75.

[Jeff85c] D. Jefferson, H. Sowizral, "Fast Concurrent Simulation Using the Time Warp Mechanism". Proceedings of the 1985 Distributed Simulation Conference, SCS simulation series, Vol.15, No.2. 1985. pp63-69.

[Jeff87] D. Jefferson, B. Beckman. F. Wieland, L. Blume, M. DiLoreto, P. Hontalas. P. Laroche, K. Sturdevant. J. Tupman, V. Warren, J. Wedel, H. Younger. S. Bellenot. “Distributed Simulation and the Time Warp Operating System". Communications ACM. 1987. pp77-93.

[Jeff90] D. Jefferson, -Virtual Time II: The Cancelback Protocol for Storage Management in Time Warp", Proceedings of the 9th Annual ACM Symposium on Principles of Distributed Computing, ACM, New York. 1990, pp75-90.

[Jha94] V. Jha, R.L. Bagrodia. "A Unified Framework for Conservative and Optimistic Distributed Simulation", Proceedings of the 8th Workshop on Parallel and Distributed Simulation. 1994. pp1219.

[Kapp95] K.L. Kapp. T.C. Hartrum. T.S. Wailes, “An Improved Cost Function for Static Partitioning of Parallel Circuit Simulations Using a Conservative Synchronization Protocol”. Proceedings of the 9th Workshop on Parallel and Distributed Simulation. 1995.

[Kell94] K. Kelly, -Out of Control: The New Biology of Machines, Social Systems. and the Economic World". Addison-Wesly Publishing Company, 1994.

[Kern70] B. W. Kernighan, S. Lin, “An Efficient Heuristic Procedure for Partitioning Graphs”. The Bell System Technical JournaL, Vol.49, No.2, February 1970, pp291-307.

[Kirk83] S. Kirkpatrick, C. Gelatt Jr., M. Vecchi, “Optimization by Simulated Annealing`. Science. Vol.220. No.4598, May 1983, pp671-680.

[Kona95] P. Konas, P.-C. Yew, “Partitioning for Synchronous Parallel Simulation". Proceedings of the 9th Workshop on Parallel and Distributed Simulation. 1995, pp181-184.

[Krav88] S.A. Kravitz, B.D. Ackland, "Static vs. Dynamic Partitioning of Circuits for a MOS Timing Simulator on a Message-Based Multiprocessor", Distributed Simulation. 1988. pp136-140. 
[Lamp78] L. Lamport, "Time, Clocks and the Ordering of Events in a Distributed System". Communications $A C M$, Vol.21, No.7, 1978, pp558-65.

[Leve82] Y.H. Levendel, P.R. Menon. S.H. Patel, "Special-Purpose Computer for Logic Simulation Using Distributed Processing". The Bell System Technical Joumal. Vol.61. No.10. December 1982, pp2873-2909.

[Lin87] F. Lin, R. Keller, "The Gradient Model Load Balancing Method". IEEE Trans. Software Engineering, Vol.13(1), 1987, pp43-52.

[Lin90] Y.-B. Lin, E.D. Lazowska, "Optimality Considerations of Time Warp Parallel Simulation". Proceedings of the 4th Workshop on Parallel and Distributed Simulation, 1990, pp29-34.

[Lin90b] Y.-B. Lin, E.D. Lazowska, "Determining the Global Virtual Time in a Distributed Simulation". Technical report 90-01-02. Department of Computer Science and Engineering. University of Washington, Seattle, WA 98195, January 1990.

[Lin90c] Y.-B. Lin, E.D. Lazowska, "Exploiting Lookahead in Parallel Simulation”. IEEE Transactions on Parallel and Distributed Systems. Vol.1, No.4, pp457-469.

[Lin91] Y.-B. Lin, E.D. Lazowska, “Processor Scheduling for Time Warp Parallel Simulation”. Proc. 1991 SCS Multiconference on Advances in Parallel and Distributed Simulations. January 1991. pp11-14.

[Lin91b] Y.-B. Lin, B.R. Preiss, “Optimal Memory Management for Time Warp Parallel Simulation”. ACM Transactions on Modeling and Computer Simulation. Vol.1. No.4. October 1991. pp96-105.

[Lin92] Y.-B. Lin, “Memory Management Algorithms for Optimistic Parallel Simulation”. Proceedings of the 6th Workshop on Parallel and Distributed Simulation, SCS simulation series, Vol.24, No.3. 1992. pp43-52.

[Lin92b] J.M. Lin, "Exploiting Dynamic Topological Information to Speed Up Concurrent Multicomputer Simulation", Proceedings of the 6th Workshop on Parallel and Distributed Simulation. 1992. pp201-202.

[Lin94] Y.-B. Lin, B.R. Preiss, W.M. Loucks, E.D. Lazowska, “Selecting the Checkpoint Interval in Time Warp Simulation", Proceedings of the 8th Workshop on Parallel and Distributed Simulation. 1994. pp3-10.

[Lipt90] R.J. Lipton, D.W. Mizell, “Time Warp vs. Chandy-Misra: A Worst-Case Comparison”, Proceedings of the \&th Workshop on Parallel and Distributed Simulation. 1990. pp137-143.

[Liu90] L.Z. Liu, C. Tropper, "Local Deadlock Detection in Distributed Simulation". Proceedings of the 1990 Distributed Simulation Conference, SCS simulation series, Vol.22, No.1. 1990, pp64-69.

[Lose85] S.L. Losen, “A Global Algorithm for the Multi-Partitioning of Graphs”. M.Sc. thesis, University of Virginia, January 1985.

[Luba88] B. Lubachevsky, "Bounded Lag Distributed Discrete Event Simulation", Proceedings of the 1988 Distributed Simulation Conference. SCS simulation series, Vol.19. No.3. February 1988, pp183191. 
[Luba89] B. Lubachevsky, A. Schwartz, A. Weiss, “Rollback Sometimes Works ... if Filtered”, Proceedings of the 1989 Winter Simulation Conference, pp630-639, December 1989

[Lux95] A. Lux. D. Steiner, “Understanding Cooperation: an Agent's Perspective”. First International Conference on Multiagent Systems. San Fransisco, California, June 1995.

[Madi90] V. Madisettim J. Walrand, D. Messerschmitt, “Synchronization in Message-Passing Computers", Proceedings of the Distributed Simulation Conference, SCS simulation series, Vol.22. No.1, pp35-48.

[Madi92] V.K. Madisetti, D.A. Hardaker. R.M. Fujimoto, "The MIMDIX Operating System for Parallel Simulation", it 6Proceedings of the th Workshop on Parallel and Distributed Simulation. SCS simulation series, Vol.24, No.3. 1992. pp65-74.

[Metr53] N. Metropolis, A. Rosenbluth, A. Teller, E. Teller, “Equation of state calculations by fast computing machines”. Journal Chem. Phys.. Vol.21. 1953, pp1087.

[Misr86] J. Misra. “Distributed Discrete-Event Simulation”. Computing Surveys, Vol.18. No.1. March 1986. pp39-65.

[Mukh86] A. Mukherjee. -Introduction to nMos and CMOS VLSI Systems Design`. Prentice-Hall International Editions, 1986.

[Naha85] S. Nahar. S. Sahni. E. Shragowitz. “Experiments with simulated annealing”. 22ns Deszgn Automation Conference, 1985, pp748-752.

[Naha86] S. Nahar. S. Sahni. E. Shragowitz. "Simulated Annealing and Combinatorial Optimization". 23rd Design Automation Conference. 1986. pp293-299.

[Nata86] N. Natarajan. “A Distributed Scheme for Detecting Communication Deadlocks”. IEEE Trans. Software Eng.. Vol. SE-12. No.4. April 1986, pp531-537.

[Ni85] L. Ni. et. al.. "A Distributed Drafting Algorithm for Load Balancing". IEEE Trans. Software Engineering. Vol.11 (10), 1985, pp16-24.

[Nico85] D.M. Nicol. P.F. Reynolds. Jr., "A Statistical Approach to Dynamic Partitioning”, Proceedings of the Distributed Simulation Conference. SCS simulation series. Vol.15. No.2, 1985. pp53-56.

[Nico88] D.M. Nicol. "High Performance Parallelized Discrete Event Simulationf of Stochastic Queuing Networks". Proceedings of the 1988 Winter Simulation Conference, pp3c6-314.

[Nobl95] B.L. Noble, R.D. Chamberlain. -Predicting the Future: Resource Requirements and Predictive Optimism". Proceedings of the 9th Workshop on Parallel and Distributed Simulation, 1995, pp157164.

[Oust83] J.K. Ousterhout. G.T. Hamachi. R.N. Mayo, W.S. Scott, G.S. Taylor, “A Collection of Papers on Magic". Rept. UCB.CSD 83/154, University of California at Berkely, Berkeley, California, December 1983.

[Page94] E.H. Page, R.E. Nance, "Parallel Discrete Event Simulation: A Modeling Methodological Perspective". Proceedings of the 8th Workshop on Parallel and Distributed Simulation, 1994, pp8893 
[Peac79] J.K. Peacock, J.W. Wong, E.G. Manning, “Distributed Simulation using a Network of Processors", Computer Networks, Vol.3, No.1, February 1979, pp44-56.

[Prei89] B.R. Preiss, "The Yaddes Distributed Discrete Event Simulation Specification Language and Execution Environment", Proceedings of the Multiconference on Distributed Simulation, 1989, pp139-144.

[Prei92] B.R. Preiss, I.D. MacIntyre, W.M. Loucks, "On the Trade-off between Time and Space in Optimistic Parallel Discrete-Event Simulation", Proceedings of the 6th Workshop on Parallel and Distributed Simulation, 1992, pp33-42.

[Prei94] B.R. Preiss. W. Loucks, I. MacIntyre, "Effects of the Checkpoint Interval on Time and Space in Time Warp", ACM Transactions on Modeling and Computer Simulation. July 1994

[Pres89] M. Presley, M. Ebling, F. Wieland. D. Jefferson, "Benchmarking the Time Warp Operating System with a Computer Network Simulation”. 3rd Workshop on Parallel and Distributed Simulation, 1989, pp24-36.

[Pres90] M.T. Presley, P.L. Reiher. S. Bellenot, *A Time Warp Implementation of the Shakrs World". Proceedings of the 1990 Winter Simulation Conference, pp199-203.

[Raja93] H. Rajaei, R. Ayani. L.-E. Thorelli, “The Local Time Warp Approach to Parallel Simulation”. Proceedings of the 7th Workshop on Parallel and Distributed Simulation, 1993. pp37-43.

[Reed88] D.A. Reed. A.D. Malony, "Parallel Discrete Event Simulation: The Chandy-Misra-Approach”. Proceedings of the SCS Multiconference on Distributed Simulation, 1988, pp8-13.

[Reed88b] D.A. Reed. A.D. Malony. A. McCredie. "Parallel Discrete Event Simulation using Shared Memory”, IEEE Trans. on Software Engineering, Vol.14, No.4. April 1988. pp541-553.

[Reih90] P.L. Reiher. D. Jefferson. -Virtual Time Based Dynamic Load Management in the Time Warp Operating System". Proceedings of the 4 th Workshop on Parallel and Distributed Simulation, 1990. pp103-111.

[Reih90b] P. Reiher, R. Fujimoto. S. Bellenot. D. Jefferson. "Cancellation Strategies in Optimistic Execution Systems". Proceedings of the Distributed Simulation Conference, SCS simulation series. Vol.22, No.1, 1990, pp112-121.

[Rome84] F. Romeo, A. Vincentelli. "Probabilistic Hill Climbing Algorithms: Properties and applications", University of California. Berkeley, UCB/ERL M84/34, 1984.

[Ronn94] R. Rönngren. R. Ayani, “Adaptive Checkpointing in Time Warp". Proceedings of the 8th Workshop on Parallel and Distributed Simulation. 1994, pp110-117.

[Russ85] G. Russel, D.J. Kinniment. E.G. Chester, M.R. McLauchlan, "CAD for VLSI", published by Van Nostrand Reinhold (UK), 1985.

[Sch195] R. Schlagenhaft, M. Ruhwandl. C. Sporrer, H. Bauer, “Dynamic Load Balancing of a MultiCluster Simulator on a Network of Workstations". Proceedings of the 9th Workshop on Parallel and Distributed Simulation, pp175-180. 
[Schu89] M.H. Schulz, F. Fink. K. Fuchs, "Parallel Pattern Fault Simulation of Path Delay Faults", 26th ACM/IEEE Design Automation Conference, June 1989, pp357-363.

[Smit87] S.P. Smith, B. Underwood, M.R. Mercer, "An Analysis of Several Approaches to Circuit Partitioning for Parallel Logic Simulation", Proceedings of the Design Automation Conference, 1987, pp664-667.

[Soko88] L.M. Sokol, D.P. Briscoe, A.P. Wieland, "MTW: A Strategy for Scheduling Discrete Simulation Events for Concurrent Execution", 2nd Workshop on Parallel and Distributed Simulation. 1988. pp34-42.

[Soko90] L.M. Sokol, B.K. Stucky, “MTW: Experimental Results For a Constrained Optimistic Scheduling Paradigm", Proceedings of the fth Workshop on Parallel and Distributed Simulation. 1990. pp169-173.

[Soul89] L. Soule, A. Gupta, "Characterization of Parallelism and Deadlocks in Distributed Digital Logic Simulation", 26th ACM/IEEE Design Automation Conference, 1989, pp81-86.

[Soul89b] L. Soule, A. Gupta, "Parallel Distributed-Time Logic Simulation”, IEEE Design $\mathcal{E}$ Test of Computers, 1989, pp32-48.

[Soul91] L. Soule, A. Gupta, "An Evaluation of the Chandy-Misra-Bryant Algorithm for Digital Logic Simulation", ACM Transactions on Modeling and Computer Simulation. Oct. 1991.

[Spor93] C. Sporrer, H. Bauer, “Corolla Partitioning for Distributed Logic Simulation of VLSICircuits", Proceedings of the 7th Workshop on Parallel and Distributed Simulation, 1993. pp85-92.

[Srin95] S. Srinivasan, P.F. Reynolds, Jr. "Super-criticality Revisited", 9th Worshop on Parallel and Distributed Simulation, Lake Placid, NY, 1995, pp130-136.

[Stan84] J. Stankovic, I. Sidhu, "An Adaptive Bidding Algorithm for Processes. Clusters, and Distributed Groups", Proc. 4th Int. Conf. Distributed Comp. Sys. 1984, pp53-61.

[Stei91] J.S. Steinman, "SPEEDES: Synchronous Parallel Environment for Emulation and Discrete Event Simulation", Proceedings of the 5th Workshop on Parallel and Distributed Simulation, 1991. pp101-109.

[Stei92] J.S. Steinman, “SPEEDES: A Unified Approach to Parallal Simulation“. Proceedings of the 6th Workshop on Parallel and Distributed Simulation 1992, pp75-84.

[Stei93] J.S. Steinman. "Breathing Time Warp", Proceedings of the 7th Workshop on Parallel and Distributed Simulation, 1993, pp109-118.

[Su89] W.-K. Su. C.L. Seitz, "Variants of the Chandy-Misra-Bryant Distributed Discrete-Event Simulation Algorithm", 3rd Workshop on Parallel and Distributed Simulation, 1989. pp38-43.

[Term83] C.J. Terman, "Simulation Tools for Digital Design", Ph.D. dissertation, Massachusetts Institute of Technology, Cambridge, Massachusetts, 1983.

[Wagn89] D.B. Wagner, E.D. Lazowska, B.N. Bershad, "Techniques for Efficient Shared-Memory Parallel Simulation", Distributed Simulation, 1989, pp29-37 
[Wald92] M. Waldrop, "Complexity: The Emerging Science at the Edge of Order and Chaos". Touchstone (Simon \& Schuster), 1992.

[Watk91] D. S. Watkins, "Fundamentals of Matrix Computations", John Wiley \& Sons Inc. 1991.

[Wiel89] F. Wieland, L. Hawley, A. Feinberg, M. Di Loreto, L. Blume, P. Reiher, B. Beckman, P. Hontalas, S. Bellenot, D. Jefferson, "Distributed Combat Simulation and Time Warp: The Model and its Performance", 3rd Workshop on Parallel and Distributed Simulation. 1989, pp14-20.

[Witt91] E. E. Witte, R. D. Chamberlain, M. A. Franklin, "Parallel Simulated Annealing Using Speculative Computation", IEEE Transactions on Parallel and Distributed Systems, Vol.2, No.4. October 1991, pp483-494.

[Wong86] K.F. Wong, M.A. Franklin, R.D. Chamberlain, B.L. Shing, “Statistics on Logic Simulation”, 23rd ACM/IEEE Design Automation Conference, 1986, pp101-107.

[Zhan92] G, Zhang, "Partitioning and Transformation of VHDL Models for Distributed Simulation". 6th Workshop on Parallel and Distributed Simulation, 1992, pp203-204.

[Zimm86] G. Zimmerman, “Top-Down Design of Digital Systems”, Logic Design and Simulation, edited by E.Hörbst, 1986, pp9-30. 
